Old Dominion University

ODU Digital Commons

Spring 2004

\title{
Spin Structure Functions of the Deuteron Measured with CLAS in and Above the Resonance Region
}

Kahanawita G. Vipuli G. Dharmawardane

Old Dominion University

Follow this and additional works at: https://digitalcommons.odu.edu/physics_etds

Part of the Nuclear Commons

\section{Recommended Citation}

Dharmawardane, Kahanawita G.. "Spin Structure Functions of the Deuteron Measured with CLAS in and Above the Resonance Region" (2004). Doctor of Philosophy (PhD), Dissertation, Physics, Old Dominion University, DOI: $10.25777 / 438 k-j v 75$

https://digitalcommons.odu.edu/physics_etds/31

This Dissertation is brought to you for free and open access by the Physics at ODU Digital Commons. It has been accepted for inclusion in Physics Theses \& Dissertations by an authorized administrator of ODU Digital Commons. For more information, please contact digitalcommons@odu.edu. 


\section{SPIN STRUCTURE FUNCTIONS OF THE DEUTERON \\ MEASURED WITH CLAS IN AND ABOVE THE RESONANCE REGION}

by

Kahanawita G. Vipuli G. Dharmawardane

B.S. June 1996, University of Colombo

A Dissertation Submitted to the Faculty of

Old Dominion University in Partial Fullfillment of the

Requirement for the Degree of

DOCTOR OF PHILOSOPHY

PHYSICS

OLD DOMINION UNIVERSITY

May 2004

Approved by:

Ga.il Dodoe (Direstor)

Jold Donat

Sebastian Kuhn

Anatoly Radvushkin

Charles I. Sukenik 


\title{
ABSTRACT \\ SPIN STRUCTURE FUNCTIONS OF THE DEUTERON MEASURED WITH CLAS IN AND ABOVE THE RESONANCE REGION
}

\author{
Kahanawita G. Vipuli G. Dharmawardane \\ Old Dominion University, 2004 \\ Director: Dr. Gail Dodge
}

Spin structure functions of the nucleon in the region of large $x$ and small to moderate $Q^{2}$ continue to be of high current interest. The first moment of the spin structure function $g_{1}, \Gamma_{1}$, goes through a rapid transition from the photon point $\left(Q^{2}=0\right)$, where it is constrained by the Gerasimov-Drell-Hearn sum rule, to the deep inelastic limit where it is sensitive to the nucleon spin fraction carried by quarks. The interesting behavior in the transition region is dominated by baryon resonance excitations. We concluded an experiment to measure these observables for deuterium as part of the "EG1" run group in Jefferson Lab's Hall B. We used a highly polarized electron beam with energies from $1.6 \mathrm{GeV}$ to $5.7 \mathrm{GeV}$ and a cryogenic polarized $\mathrm{ND}_{3}$ target together with the CEBAF Large Acceptance Spectrometer (CLAS) to accumulate over 11 billion events. In this thesis, we present results for the spin structure function $g_{1}^{d}\left(x, Q^{2}\right)$, as well as its first moment, $\Gamma_{1}^{d}\left(Q^{2}\right)$ in and above the resonance region over a $Q^{2}$ range from 0.05 to $5 \mathrm{GeV}^{2}$, based on the data taken with beam energies of 1.6 and $5.7 \mathrm{GeV}$. We also extract the behavior of $A_{1}^{d}(x)$ at large x. Our data are consistent with the Hyperfine-perturbed quark model calculation which predicts that $A_{1}^{d}(x \rightarrow 1) \rightarrow 1$. We also see evidence for duality in $g_{1}^{d}\left(x, Q^{2}\right)$ at $Q^{2}>1.5 \mathrm{GeV}^{2}$. 
(C)Copyright, 2004, by Kahanawita G. Vipuli G. Dharmawardane, All Rights Reserved 


\section{ACKNOWLEDGMENTS}

This work could have not been possible without the help and support of many different people. First, I would like to acknowledge the assistance provided by the Jefferson Lab staff, the CLAS Collaboration and ODU physics department. This research was supported by the US Department of Energy under the grant DE-FG02-96ER40960.

I would like to thank my advisor, Gail Dodge, for her support throughout the duration. I would specially like to thank her for revising this thesis. A very special thanks go to Sebastian Kuhn, who provided useful advise, direction and invaluable assistance throughout the course of this thesis. I would also like to thank John Donat, Charles Sukenik and Anatoly Radyushkin for serving on my thesis committee.

This thesis is a result of the dedication of a great number of people. In particular I would like to thank Peter Bosted for his guidance. I also had the pleasure of working with Shifeng Chen, Yelena Prok, Raffaella De Vita, Angela Biselli, Volker Burkert, Keith Griffioen, Ralph Minehart, Harut Avakian and Tony Forest. I benefitted enormously from their willingness to help. I would also like to thank Wally Melnitchouk and Professor Winston Roberts for providing me insight and assistance on theoretical issues. I thank Chris Keith for his quick responses to all my EG1 target related questions. I would like to thank David Lawrence, Mac Mestayer and Stepan Stepanyan from whom I learned so much during my early years.

I would like to thank my fellow students, Rustam Niyazov, Junho Yun, Mehmet Bektasoglu, Prasong Kessaratikoon, Changkun Dong, David Hayes, Sharon Careccia, Alexei Klimenkov, Ales Psaker, Nevzat Guler, Ruben Iglloria and Hovhannes Baghdasaryan for making my stay at graduate school a pleasant one. I would specially like to thank Luminita Todor for always being there as a friend. I wish to thank Fatiha Benmokhtar for her friendship and good food. I thank Tom Hartlove for always being kind and understanding.

I would like to thank my parents for giving me the freedom to let me choose my own goals. I can not thank them enough for their love and support. I would specially like to thank them for always being there on the other side of the phone whenever I needed them. 


\section{TABLE OF CONTENTS}

Page

List of Tables ........................ . . . . . . . . .

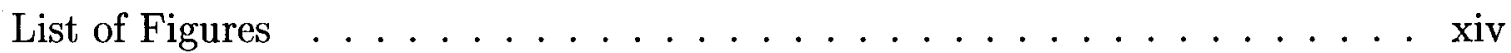

\section{CHAPTERS}

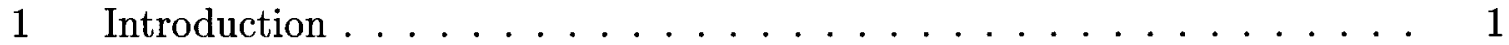

2 Formalism and Physics Overview ................ 4

2.1 Lepton Hadron Scattering . . . . . . . . . . . . . . . . 4

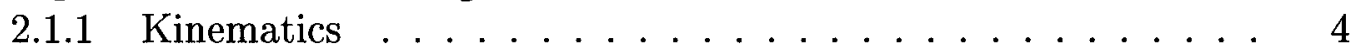

2.1.2 Formalism in One Photon Exchange .......... 5

2.1.3 Virtual Photon Asymmetries . . . . . . . . . . 7

2.2 Spin Structure Functions . . . . . . . . . . . . . . . . . 9

2.2.1 Parton Model Interpretation of Structure Functions . . . . . . 10

2.2.2 $Q^{2}$ Dependence of the Spin Structure Function $g_{1} \ldots \ldots 11$

2.2 .3 Resonance Contribution to $g_{1} \ldots \ldots \ldots 15$

2.2 .4 Spin Structure Function $g_{2} \ldots \ldots \ldots 18$

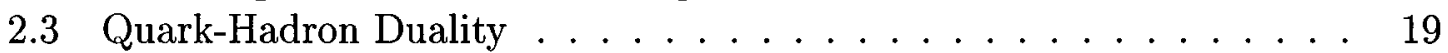

$2.4 A_{1}$ in the Valence Region . . . . . . . . . . . . . . . 22

2.4.1 $A_{1}$ in the Parton Model . . . . . . . . . . 23

2.4 Predictions for $A_{1} \ldots \ldots \ldots 24$

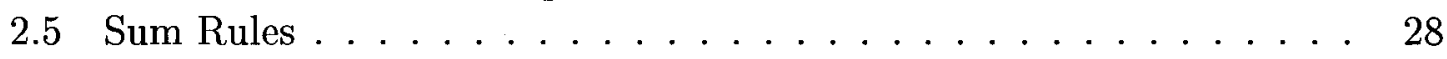

2.5.1 Sum Rules at Kinematic End Points . . . . . . . . . . 28

2.5 .2 Generalized Sum Rules for $\Gamma_{1} \ldots \ldots . \ldots 30$

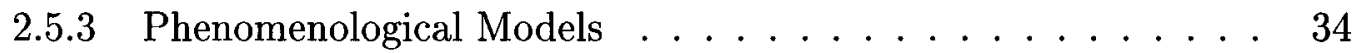

2.6 Deuteron as a Proton+neutron Target . . . . . . . . . . . . 37

2.6.1 D State Correction . . . . . . . . . . . . . . . 37

2.6.2 Nuclear-binding Effects . . . . . . . . . . . . . . . 39

3 Experimental Setup . . . . . . . . . . . . . . . . . . 41

3.1 The Accelerator and the Polarized Beam . . . . . . . . . . . . 41

3.2 Hall B Beam Line Devices . . . . . . . . . . . . . . . . . . . . 44

3.3 The CLAS . . . . . . . . . . . . . . . . . . 46

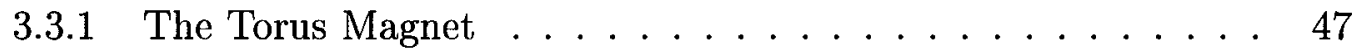

3.3 .2 Drift Chambers . . . . . . . . . . . . . . 48

3.3.3 Time of Flight Counters . . . . . . . . . . . . 49

3.3.4 Cerenkov Counters . . . . . . . . . . . . . . 50

3.3.5 Electromagnetic Calorimeter .............. 51

3.4 Polarized Target . . . . . . . . . . . . . . . . . . 53

3.4.1 The Target System . . . . . . . . . . . . . . . 53

3.4.2 Polarized Target Materials .............. . 54

3.4.3 Dynamic Nuclear Polarization . . . . . . . . . . . . . 55

3.4.4 Monitoring the Target Polarization ............ 58 
3.5 The Trigger and the Data Acquisition System . . . . . . . . . 61

4 Event Reconstruction . . . . . . . . . . . . . . . . . . . . . . . 64

4.1 Track Reconstruction . . . . . . . . . . . . . . . . . . . 64

4.1.1 Finding a Particle Track . . . . . . . . . . . . . . 64

4.1.2 Reconstruction of the Start Time . . . . . . . . . . 67

4.2 Time Based Tracking . . . . . . . . . . . . . . . 70

$5 \quad$ Data Analysis . . . . . . . . . . . . . . . . 75

5.1 Beam Settings . . . . . . . . . . . . . . 75

5.2 Run Selection . . . . . . . . . . . . . . . 76

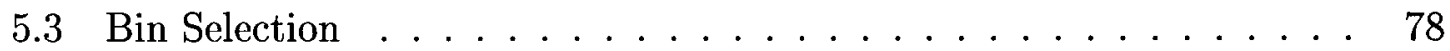

$5.4 \quad$ Fiducial Cuts . . . . . . . . . . . . . . . . . 78

5.5 Electron Identification $\ldots \ldots \ldots \ldots \ldots \ldots$

5.5 .1 Cerenkov Cut . . . . . . . . . . . . . . 86

5.5.2 Electromagnetic Calorimeter Cuts . . . . . . . . . . . . 88

5.5.3 Raster Correction and Vertex Cut . . . . . . . . . . . . 90

5.6 Momentum Correction . . . . . . . . . . . . . . . . . 92

5.7 Extraction of the Asymmetry $A_{\|} \ldots \ldots \ldots \ldots \ldots$

5.7 .1 Raw Asymmetry . . . . . . . . . . . . . . 97

5.7 .2 Dilution Factor . . . . . . . . . . . . . . . . . . 98

5.7 .3 Backgrounds . . . . . . . . . . . . . . . 109

5.7 .4 Electroweak Asymmetry . . . . . . . . . . . . . . . . 113

5.7.5 Beam and Target Polarization . . . . . . . . . . . . . 114

5.7.6 Polarized Nitrogen and Residual Proton Correction . . . . . 120

5.7.7 Radiative Corrections . . . . . . . . . . . . . . . . . 122

5.8 Model Input . . . . . . . . . . . . . . . . . . . . . 125

5.9 Combining Different Groups of Runs _ . . . . . . . . . . . . . 127

5.10 Systematic Errors . . . . . . . . . . . . . . . . . . 131

5.10 .1 Dilution Factor . . . . . . . . . . . . . . . . 132

5.10 .2 Background Processes . . . . . . . . . . . . . . 134

5.10 .3 Polarized Nitrogen and Proton Correction . . . . . . . . 134

5.10 .4 Beam and Target Polarization . . . . . . . . . . . . 135

5.10 .5 Systematic Errors due to Models . . . . . . . . . . . . 135

$6 \quad$ Results . . . . . . . . . . . . . . . . . . . 143

6.1 Asymmetry $A_{1}+\eta A_{2} \ldots \ldots \ldots \ldots \ldots \ldots \ldots \ldots \ldots$

6.2 Spin Structure Function $g_{1} \ldots \ldots \ldots \ldots \ldots \ldots \ldots$

6.3 Duality . . . . . . . . . . . . . . . . . 146

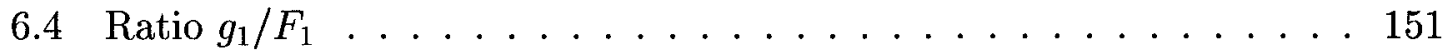

6.5 Virtual Photon Asymmetry $A_{1} \ldots \ldots \ldots \ldots \ldots \ldots$

6.6 The First Moment of $g_{1} \ldots \ldots \ldots \ldots \ldots \ldots \ldots$

6.6.1 Low $Q^{2}$ Region . . . . . . . . . . . . . . 156

6.6 .2 High $Q^{2}$ Region . . . . . . . . . . . . 156 


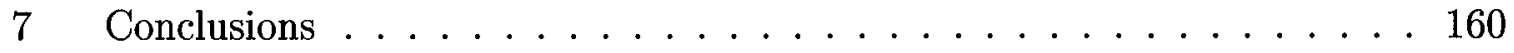

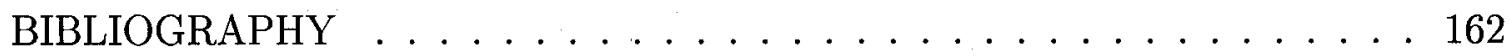

\section{APPENDICES}

A DATA TABLES FOR $g_{1}(\mathrm{E}=1 . \mathrm{x} \mathrm{GeV}) \ldots \ldots \ldots \ldots \ldots$

B DATA TABLES FOR $g_{1}(\mathrm{E}=5 \cdot \mathrm{x} \mathrm{GeV}) \ldots \ldots \ldots \ldots \ldots$

C DATA TABLES FOR $A_{1}, g_{1} / F_{1}$ and $\Gamma_{1} \ldots \ldots \ldots \ldots \ldots$

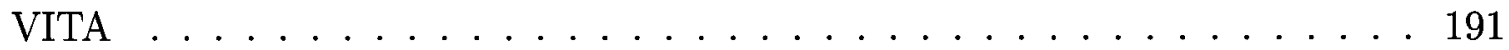




\section{LIST OF TABLES}

\begin{tabular}{|c|c|c|}
\hline & & \\
\hline & le quantum numbers of the $\mathrm{SU}(3)$ group. . . . . . . . . & \\
\hline & mmary of data analyzed in this thesis. . . . . . . . & 76 \\
\hline & The table lists the $Q^{2}$ bins used in the analysis. & \\
\hline & & 9 \\
\hline & 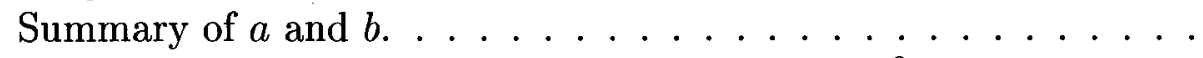 & 102 \\
\hline & The table lists the high $W$ cut applied to each $Q^{2}$ bin. . & 107 \\
\hline & $\begin{array}{l}\text { Comparison of } A_{\text {quasi-el }}^{d} \text { obtained using two different methods for } \\
\text { the } 5.735 \mathrm{GeV} \text { outbending data. . . . . . . . . . }\end{array}$ & 116 \\
\hline & $\mathrm{d}[62]$ using a simulation for different $Q^{2}$ bins. . & 117 \\
\hline & & 120 \\
\hline & ....... & 129 \\
\hline & $\mathrm{eV}$ data. . . . . . . . . . & 130 \\
\hline & of the $x$ bins used in the calculation & 152 \\
\hline & 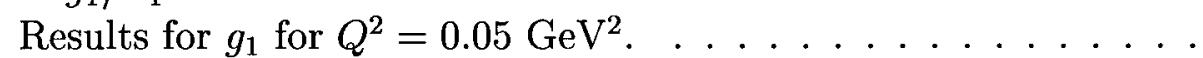 & 16 \\
\hline & $\mathrm{GeV}^{2}$. & 168 \\
\hline & $Q^{2}=0.0$ & 169 \\
\hline & $\mathrm{GeV}^{2} \ldots \ldots \ldots$ & 169 \\
\hline & $V^{2} \ldots \ldots$ & 170 \\
\hline & $\ldots \ldots \ldots$ & 170 \\
\hline & $\ldots \ldots . . .$. & 171 \\
\hline & .......... & 171 \\
\hline & $\cdots \cdots$ & 172 \\
\hline & $Q^{2}=0$ & 173 \\
\hline & $Q^{2}=0$ & 174 \\
\hline & $\mathrm{GeV}^{2}$. & 174 \\
\hline & $\mathrm{GeV}^{2} \ldots \ldots \ldots$ & 175 \\
\hline & $\mathrm{GeV}^{2} \ldots \ldots \ldots$ & 175 \\
\hline & ${ }_{1}$ for $Q^{2}=0.6 \mathrm{GeV}^{2} \ldots \ldots \ldots$ & 176 \\
\hline & or $g_{1}$ for $Q^{2}=0.84 \mathrm{GeV}^{2} \ldots \ldots$ & 178 \\
\hline & ${ }_{1}$ for $Q^{2}=1.0 \mathrm{GeV}^{2} \ldots \ldots \ldots$ & \\
\hline & for $g_{1}$ for $Q^{2}=1.2 \mathrm{GeV}^{2} \ldots \ldots \ldots$ & 180 \\
\hline & $g_{1}$ for $Q^{2}=1.4 \mathrm{GeV}^{2}$. & \\
\hline & $\mathrm{s}$ for $g_{1}$ for $Q^{2}=1.7 \mathrm{GeV}^{2}$. & 182 \\
\hline & for $Q^{2}=2.0 \mathrm{GeV}^{2}$. & 183 \\
\hline & Resu & 183 \\
\hline & $Q^{2}=3.0 \mathrm{GeV}^{2}$ & 184 \\
\hline & ts for $g_{1}$ for $Q^{2}=3$ & 184 \\
\hline & & \\
\hline & 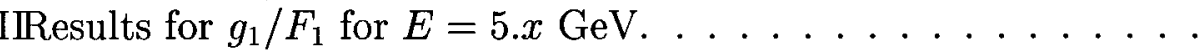 & \\
\hline
\end{tabular}


XXXIX Results for averaged $A_{1}$ for $Q^{2}$ between 1.4 and $4.52 \mathrm{GeV}^{2}$ and $W>1.6 \mathrm{GeV} \ldots \ldots \ldots \ldots \ldots \ldots \ldots \ldots$

XL Results for $\Gamma_{1}^{d}$ integrated up to the values in $W$ given in table VI (measured region). . . . . . . . . . . . . . 189

XLI $\quad \Gamma_{1}^{d}$ in the measured region plus the DIS contribution given by our model. . . . . . . . . . . . . . . . . . . . . . 190 


\section{LIST OF FIGURES}

1 First order Feynman diagram for lepton nucleon scattering. . . . . . . 5

2 Parton model description of deep inelastic electron nucleon scattering. 10

3 Deep inelastic scattering world data on $g_{1}^{p}$ [13] as of 1999. . . . . . . 11

4 Leading order and next to leading order contributions to electron scattering from a quark. ................. 12

5 Vertices which determine the splitting functions. . . . . . . . . . 13

6 Feynman diagrams that contribute to higher twist effects. . . . . . . . 14

$7 \quad$ Cross section for the inclusive reaction $e p \longrightarrow e^{\prime} X$ at $Q^{2}=1.4 \mathrm{GeV}^{2} . \quad 15$

8 The ratios $E_{1+} / M_{1+}, S_{1+} / M_{1+}$ for $\gamma^{*} p \Delta(1232)[20] \ldots \ldots$. . . . . 17

9 Measurement of $x g_{2}^{p}$ and $x g_{2}^{d}[24] \ldots \ldots \ldots 18$

10 The spin independent structure function $\nu W_{2}$ plotted as a function of $\xi$ for hydrogen at different $Q^{2}$ values $[27] \ldots \ldots$. . . . . . . . . 19

11 Different kinematic regions where (A) higher twist effects are negligible, (B) higher twist effects are non-negligible however are small and (C) higher twist effects are large and nonperturbative [19]. . . . . . . 20

12 Parton distributions at $Q^{2}=5 \mathrm{GeV}^{2}$ in CTEQ5M [30] parameterization. 22

13 Diagram of polarized deep inelastic scattering. . . . . . . . . . 23

14 Predictions for $A_{1}^{d}$ in the valence region. . . . . . . . . . . . 26

15 Two diagrams describing the transfer of momenta from the spectator quark pair to the struck quark as $x \rightarrow 1$. . . . . . . . . . 27

16 Forward virtual Compton scattering amplitude. . . . . . . . . 31

17 The plot shows higher twist calculations done by Ji and Melnitchouk

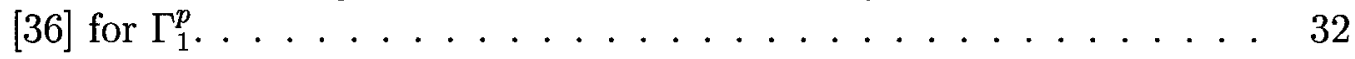

18 One-loop diagrams which contribute to $\chi \mathrm{PT}$ calculations (leading order). 34

19 Diagrams representing formulations of the VDM. . . . . . . . 35

20 Predictions for $\Gamma_{1}^{d} \ldots \ldots \ldots \ldots \ldots$

21 Spin of the deuteron as a combination of the proton and the neutron spin. . . . . . . . . . . . . . . . . 37

22 Kinematic coverage of the EG1a data [40] and the EG1b data. . . . . 40

23 The Thomas Jefferson National Accelerator Facility. . . . . . . . . 41

24 Charge distribution in the RF cavities at one instant. . . . . . . . . . 42

25 The energy level diagrams for GaAs grown on a combination of GaAs. 43

26 The X profile of the beam obtained using a Harp scan [41] . . . . . . 44

27 A view from the top of the Hall B Moller polarimeter [41]. . . . . . . 45

28 A cutaway view of the CLAS. . . . . . . . . . . . . . 46

29 Constant magnetic field contours for the CLAS torus at a current setting of $3860 \mathrm{~A}$ [41]. . . . . . . . . . . . . . . . . 47

30 Midplane slice of the CLAS, showing the region one, two and three DCs and other detectors [42]. . . . . . . . . . . . . . . . 48

31 One drift chamber sector showing the wire direction. . . . . . . . . . 49

32 Time of flight counters for one sector. . . . . . . . . . . . . 50 
33 A schematic of a Cerenkov segment. . . . . . . . . . . . . . 51

34 Exploded view of CLAS electromagnetic calorimeter module for one sector [45]. . . . . . . . . . . . . . . . . . 52

35 A side-view of the EG1 polarized target system [46] . . . . . . . . 54

36 A schematic of the target insert used during the EG1 run. . . . . . . 55

37 The energy level diagram of a spin $1 / 2$ nucleon electron system that is placed in a magnetic field. . . . . . . . . . . . . . 57

38 The transition between energy levels that are used in DNP. . . . . . 58

39 A diagram of the NMR circuit [46] . . . . . . . . . . . . . 59

40 An enhanced polarization NMR signal for $\mathrm{ND}_{3} \ldots \ldots \ldots 60$

41 Polarization measured by the NMR for different runs [46]. . . . . . 61

42 A schematic of the CLAS data flow. . . . . . . . . . . . . . . 62

43 A hit in the EC. . . . . . . . . . . . . . . 66

44 Invariant mass of $\pi^{0}$ and $\eta$, reconstructed using two photon events recorded in the EC [41]. . . . . . . . . . . . . . . . 67

45 The RF offset for one EG1 run. . . . . . . . . . . . . . 68

46 The RF offset vs RF time should not show any RF dependence when the TOF is properly calibrated. . . . . . . . . . . . . . . . 69

47 The mean of the RF offset (in ns) as a function of the TOF paddle number for one EG1 run. . . . . . . . . . . . . . . . . 69

48 A TBT track through a single superlayer. . . . . . . . . . . . 70

49 The contours of constants time and electric field lines for a Region 3 (R3) and a Region 2 (R2) cell drawn using the GARFIELD program $[42] . \ldots \ldots \ldots \ldots \ldots \ldots . \ldots \ldots$

50 The DOCA $(\mathrm{cm})$ as a function of drift time (ns) for Sector 1 Superlayer $6[51] . \ldots \ldots \ldots \ldots \ldots \ldots$

51 DC residuals vs calculated DOCA for the three regions for sector 1 for one EG1 run. . . . . . . . . . . . . . . . . . . 73

52 DC resolution, which is the sigma of a double Gaussian fit to residuals, as a function of run number for the $5.6 \mathrm{GeV}$ inbending data. . . . . . 74

53 The plot shows the kinematic coverage of the $5 . \mathrm{x} \mathrm{GeV}$ and $1 . \mathrm{x} \mathrm{GeV}$ data. ............................ 75

54 The inclusive electron rates for each sector as a function of the file number [52]. . . . . . . . . . . . . . . . . . 77

55 Integrated asymmetry as a function of run number for the $5.627 \mathrm{GeV}$

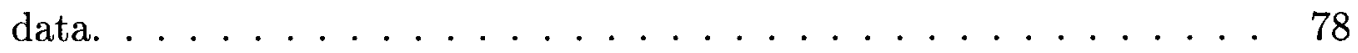

56 The plot shows the number of events scattered as a function of raster $x$ position and raster $y$ position. . . . . . . . . . . .

57 The number of photoelectrons vs $\phi_{D C}$ vs $\theta_{D C}$ for momentum between 1 and $3 \mathrm{GeV} . \ldots \ldots \ldots 1$

58 Comparison of the $\phi$ angle at the vertex (left) and the $\phi$ angle at the DC layer 1 (right) as a function of $\theta \ldots \ldots \ldots$

59 The number of events scattered from the regions where the Cerenkov efficiency is greater than $80 \%$. . . . . . . . . . . 83 
60 Same as Fig. 57 but for outbending torus current. . . . . . . . . . . . 84

61 Fiducial cuts for outbending torus settings. . . . . . . . . . . 85

62 The number of photoelectrons detected in the Cerenkov counter for $5.625 \mathrm{GeV}$ momentum $<3 \mathrm{GeV}$ data. . . . . . . . . . . . 86

63 The upper plot shows the rf offset vs the number of photoelectrons

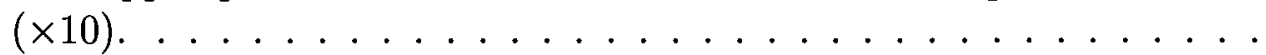

64 The reconstructed TOF mass after applying different photoelectron cuts. ........................ 88

$E_{\text {total }} / P_{e l}$ vs $E_{\text {inner }} / P_{e l}$ for different momentum bins. . . . . . . . . 89

66 Electromagnetic calorimeter cuts. . . . . . . . . . . . 90

67 The fit to the coefficients $X_{0}, Y_{0}, c_{x}$ and $c_{y}$ as a function of the run

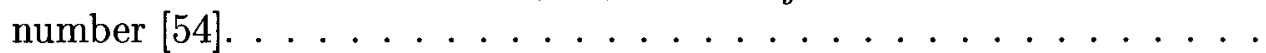

68 The figure shows the $\phi$ angle vs the $\mathrm{z}$ vertex position before (left) and after (right) applying the raster correction. . . . . . . . . . . .

69 After applying the raster correction a cut indicated by the two vertical lines was applied to select electrons scattered from the target. . . . .

70 The nominal value of the elastic peak position divided by the observed value as a function of the azimuthal angle $\phi$ for all six sectors [52]. . . The missing four momenta of events within $-1<\left|\phi_{e}-\phi_{p}\right|-180<1$. W distribution for inclusive electron scattering before (left) and after applying the momentum correction (right). . . . . . . . . . . 96 Same as Fig. 70 but after applying the momentum correction [52]. . . The structure of the beam helicity and the sync. . . . . . . . . . 98

75 The ratio of normalized ${ }^{4} \mathrm{He}$ and ${ }^{12} \mathrm{C}$ spectra for one $Q^{2}$ bin at the beam energy $5.6 \mathrm{GeV}$. . . . . . . . . . . . . 100 To obtain parameters $a$ and $b$, a global fit to all $Q^{2}$ and $W$ bins was performed. . . . . . . . . . . . . . . . . . 102

77 Comparison of the simulated ${ }^{15} \mathrm{~N}$ spectrum and the ${ }^{12} \mathrm{C}$ spectrum. . . 103

78 The figure shows the target length $l_{A}$ vs $W \ldots \ldots \ldots$. . . . . 103

79 The figure (top) shows the $\mathrm{ND}_{3}$ spectrum, the constructed total background and the deuteron spectrum. . . . . . . . . . . . 104

80 The dilution factor for all six sectors for one $Q^{2}$ bin. . . . . . . . . 105

81 The figure shows the $\mathrm{ND}_{3} /{ }^{12} \mathrm{C}$ ratio obtained using data and calculated using a model (dashed line) [57]. . . . . . . . . . . . . . . . 106

82 The dilution factor plotted as a function of $W$ for $1.6 \mathrm{GeV}$ data. . . . 108

83 The dilution factor plotted as a function of $W$ for the $5.6 \mathrm{GeV}$ data. . 108

84 The Cerenkov photoelectron spectrum was used to determine the pion contamination. . . . . . . . . . . . . . 109

85 The photoelectron spectrum and a well defined pion spectrum which has been normalized to the difference shown in Fig. 84. . . . . . . . . 110

86 Pion contamination vs momentum for one $\theta$ bin. . . . . . . . . . . 111

87 Pion contamination as a function of momentum extracted using the method described in the text for different $Q^{2}$ values. . . . . . . . . . 112 
88 The ratio $e^{+} / e^{-}$extracted using two different methods as a function of momentum for three theta bins. . . . . . . . . . . . . 113

89 Target polarization as a function of run number. . . . . . . . . . 115

90 The product of beam and target polarization was extracted by fitting the product extracted for each $Q^{2}$ bin with a constant function. . . . 118

$91 \quad \mathrm{ND}_{3} W$ spectrum obtained using ep coincidences for $Q^{2}=2 \mathrm{GeV}^{2}$. . 119

92 Comparison of $P_{b} P_{t}$ values extracted using the two methods described in the text for the $5.627 \mathrm{GeV}$ inbending data. . . . . . . . . . . . 119

93 The figure shows the Feynman diagrams for internal radiative corrections. . . . . . . . . . . . . . . . . . . . . 123

94 The figure shows $A_{\|}^{\text {rad }}$ and $A_{\|}^{B o r n}$ as a function of $W$ for a typical $Q^{2}$ bin. . . . . . . . . . . . . . . . . . . . 125

95 Plot shows the z-score values for different data sets. . . . . . . . . . . 131

96 The effect on the dilution factor due to different sources of systematic errors. . . . . . . . . . . . . . . . . . . . . 132

97 The $\sigma_{n} / \sigma_{D}$ values that were used in the analysis. . . . . . . . 133

98 The systematic error on $g_{1}$ due to the dilution factor compared to the statistical uncertainty for one $Q^{2}$ bin. . . . . . . . . . . 134

99 Different parameterizations of $A_{2}$ used to calculate systematic errors. 136

100 Measurement of $A_{2}$ for the deuteron and proton [24] . . . . . . 137

101 The total systematic uncertainty due to models used to extract asymmetries compared to the statistical uncertainty for one $Q^{2}$ bin. . . 138

102 The total systematic error given in Fig. 101, decomposed into different models used to determine the total error. . . . . . . . . . . . . 138

103 Different parameterizations of $R$ used to calculate systematic errors. . 139

104 Different parameterizations of $F_{1}$ used to calculate systematic errors. 140

105 The total systematic uncertainty due to modeling of structure functions compared to the statistical uncertainty for one $Q^{2}$ bin. . . . . 141

106 The total systematic error given in Fig. 105, decomposed into different models used to determine the total error. . . . . . . . . . . . . 141

107 The asymmetry $A_{1}+\eta A_{2}$ for the $1.6 \mathrm{GeV}$ data. . . . . . . . 143

108 The asymmetry $A_{1}+\eta A_{2}$ for $5 . \mathrm{x} \mathrm{GeV} \mathrm{data.} \mathrm{.} \mathrm{.} \mathrm{.} \mathrm{.} \mathrm{.} \mathrm{.} \mathrm{.} \mathrm{.} \mathrm{.} \mathrm{.} 144$

109 The figure compares $g_{1}$ and $A_{1}$ with the contribution from the unmeasured part. . . . . . . . . . . . . . . . 145

$110 g_{1}$ as a function of $x$ for $Q^{2}=0.05-0.17 \mathrm{GeV}^{2} \ldots \ldots \ldots \ldots$

111 Same as Fig. 110 but for $Q^{2}=0.2-0.5 \mathrm{GeV}^{2}$. . . . . . . . . . . 147

112 Same as Fig. 110 but for $Q^{2}=0.84-3.0 \mathrm{GeV}^{2} \ldots \ldots \ldots \ldots \ldots . \ldots 147$

113 Spin structure function $g_{1}$ as a function of the Natchmann variable $\xi$. 148

114 A comparison between the scaling structure function calculated using the GRSV2000 LO and NLO calculations [80] and a parameterization of world data at $Q^{2}=10 \mathrm{GeV}^{2} \ldots \ldots \ldots \ldots \ldots$

115 The ratio $I_{R E S / D I S}$ in the entire resonance region. . . . . . . . 150

116 The ratio $I_{R E S / D I S}$ calculated in the three prominent resonance enhancement regions given in the text. 
117 The figure shows the $Q^{2}$ evolution of the ratio $g_{1} / F_{1}$ for the deuteron. 152

118 The virtual photon asymmetry after correcting for the D state as a function of $x \ldots \ldots \ldots \ldots \ldots \ldots \ldots \ldots$

119 Comparison of $\Gamma_{1}$ to models described in the second chapter. . . . . 155

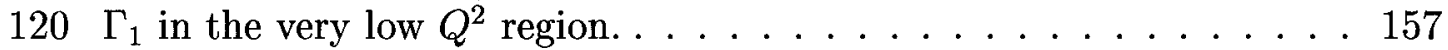

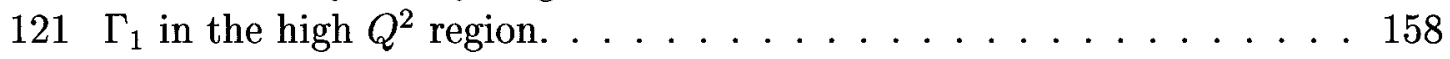




\section{CHAPTER 1}

\section{INTRODUCTION}

The best known tool to probe the nucleon structure is lepton-nucleon scattering. The short distance structure of the nucleon can be revealed by scattering highly energetic electrons, which emit virtual photons that are observed by the nucleon. Photons which have relatively low energy and momentum probe the excited states of the nucleon, known as resonances. High energy photons, on the other hand, can be absorbed by a single constituent of the nucleon in a process known as Deep Inelastic Scattering (DIS). Since the DIS experiments carried out in late 1960s and early 1970s confirmed the existence of a nucleon substructure, there has been a large interest in explaining the spin structure of the nucleon in terms of its constituents, quarks and gluons. In a simple nonrelativistic model of the nucleon, one would expect the quarks to carry the entire spin of the nucleon. One of the early theories that explained the partonic substructure of the nucleon, the Naive Parton Model, predicted that $60 \%$ of the nucleon spin is carried by the quarks.

During the last two decades much progress has been made in polarized beam and target technologies, making it possible to experimentally extract information on the shape and magnitude of the spin-dependent structure functions, $g_{1}$ and $g_{2}$, which are related to the spin carried by the quarks in the nucleon. One of the first experiments carried out at Stanford Linear Accelerator Center (SLAC), in a limited kinematic region, seemed to confirm the Naive Parton Model predictions. However subsequent measurements in a larger kinematic region performed by the European Muon Collaboration (EMC) experiment at Counseil Europáen pour la Nucléaire (CERN) reported that, contrary to the Naive Parton Model predictions, only $12 \pm 17 \%$ [1] of the spin is carried by quarks. This discovery, referred to as the "spin crisis", sparked a large interest in measuring the spin content of the nucleon and gave birth to several experiments. Since then experiments dedicated to measuring the spin fraction carried by quarks have been conducted at SLAC, CERN and DESY (Deutsches ElectronenSynchrotron). The subsequent theoretical developments of Quantum Chromodynamics (QCD) have clarified our picture of the nucleon spin structure in great detail. The Bjorken sum rule [2], which relates results of the inclusive, polarized deep inelastic

This dissertation follows the form of The Physical Review. 
lepton-nucleon scattering to the fundamental axial coupling constant, is a precise test of QCD. The interpretation of existing DIS results has verified the Bjorken sum rule at the level of $10 \%$ accuracy and has shown that only about $31 \pm 10 \%$ [3] of the nucleon spin is carried by the quarks; the rest of the spin must reside either in gluons or orbital angular momentum of its constituents. Experiments to measure the gluon contribution are underway at DESY, BNL (Brookhaven National Laboratory) and CERN.

Probing the nucleon structure at the other end of the energy scale provides information about the long distance structure which is associated with static properties of the nucleon. At the real photon point, the Gerasimov-Drell-Hearn (GDH) sum rule [4], which is based on very general principles, relates the total cross section of polarized photons on polarized nucleons with the anomalous magnetic moment of the nucleon. Although the GDH sum rule was formulated in the 1960's, it remained unappreciated until Anselmino et al. [5] pointed out the importance of the sum rule in an attempt to solve the "spin crisis." They showed that the GDH sum rule is intimately connected to the DIS region and, in fact, is the analytic extension of the Bjorken sum rule towards the real photon point. It implies a negative slope on $\Gamma_{1}$, the first moment of the spin structure function $g_{1}$, at the photoabsorption point. Later, Burkert et al. [6] pointed out that the rapid transition of $\Gamma_{1}$ between the real photon point and the DIS region is saturated by contributions from nucleon resonances. Since then Ji et al. [7] have extended the GDH sum rule beyond the real photon point.

This progress in theoretical work has triggered a large interest in measuring the spin structure functions and their moments in this relatively unexplored transition regime, between the real photon point and the DIS region. There is a large experimental program underway at Jefferson Laboratory in Newport News, VA to make precise measurements in this region. Experment E93-009, "The polarized structure function $G_{1 n}$ and the $Q^{2}$ dependence of the Gerasimov-Drell-Hearn sum rule for the neutron" [8], was performed in Hall B with the goal of measuring the spin structure function $g_{1}$ and its first moment over a large kinematic region which includes the transition region and complements the real photon point and the already measured DIS region. The main goal of this thesis is to analyze and evaluate the data of experiment E93-009 taken at beam energies of 1.x and 5.x GeV (x stands for slightly different beam energies) on polarized deuteron targets. The topics that can be studied with 
this enormous data set include an experimental verification of chiral perturbation theory and future lattice QCD calculations for $\Gamma_{1}$, an evaluation of higher twist effects for moments of $g_{1}$, the presence or absence of quark-hadron duality in the spin structure function $g_{1}$ and the behavior of the virtual photon asymmetry $A_{1}$ at large Bjorken $x$. This thesis is organized as follows. The formalism of polarized leptonnucleon scattering and a detailed overview of the subjects mentioned above will be discussed in the second chapter. In the third chapter, a brief overview of the experimental setup is given. The fourth and the fifth chapters mainly focus on the details of the analysis, including reconstruction of particle tracks from raw data. Finally, the results and the conclusions are given in chapters six and seven. 


\section{CHAPTER 2}

\section{FORMALISM AND PHYSICS OVERVIEW}

\subsection{LEPTON HADRON SCATTERING}

To observe the internal structure of hadrons, a probe which has a wave length smaller than the size of the object that is being observed has to be used. One of the best understood ways of probing the internal structure of the nucleon is through lepton scattering. The main process that will be discussed in this chapter is shown in Fig. 1 , where an incoming electron emits a virtual photon which is then absorbed by a nucleon. In inclusive measurements only the scattered lepton is detected, whereas additional final state particles are detected for exclusive reactions. In this section the formalism of polarized deep inelastic scattering for the inclusive reaction $e+N \longrightarrow$ $e^{\prime}+X$ will be discussed.

\subsubsection{Kinematics}

The electromagnetic interaction of a lepton with a nucleon is described by the exchange of virtual photons. Fig. 1 shows the Feynman diagram of the scattering of a lepton off a nucleon in the one photon exchange. All kinematic variables are defined in the lab frame. $k=\left(E, k_{x}, k_{y}, k_{z}\right), k^{\prime}=\left(E^{\prime}, k_{x}^{\prime}, k_{y}^{\prime}, k_{z}^{\prime}\right), p=(M, 0,0,0)$ and $q=\left(\nu, q_{x}, q_{y}, q_{z}\right)$ are the four momenta of the incoming lepton, outgoing lepton, target nucleon, and virtual photon. $\mathrm{M}$ is the target nucleon mass and $s$ and $s^{\prime}$ are the covariant spin four-vectors of the incoming and outgoing lepton, where $s \cdot k=s^{\prime} \cdot k^{\prime}=0$ and $s \cdot s=s^{\prime} \cdot s^{\prime}=-1$.

Several useful quantities can be defined from $k, k^{\prime}, p$ and $q$ :

- $Q^{2}=-q^{2}=2 E E^{\prime} \sin ^{2} \theta / 2$, where $\theta$ is the scattering angle of the lepton relative to the incoming direction.

- The energy of the virtual photon $\nu=E-E^{\prime}=\frac{p \cdot q}{M}$.

- The invariant mass $W^{2}=(p+q)^{2}=M^{2}+2 M \nu-Q^{2}$.

- Bjorken scaling variable $x=\frac{Q^{2}}{2 p \cdot q}=\frac{Q^{2}}{2 M \nu}$.

- $y=\frac{p \cdot\left(k-k^{\prime}\right)}{p \cdot k}=\frac{E-E^{\prime}}{E}$. 


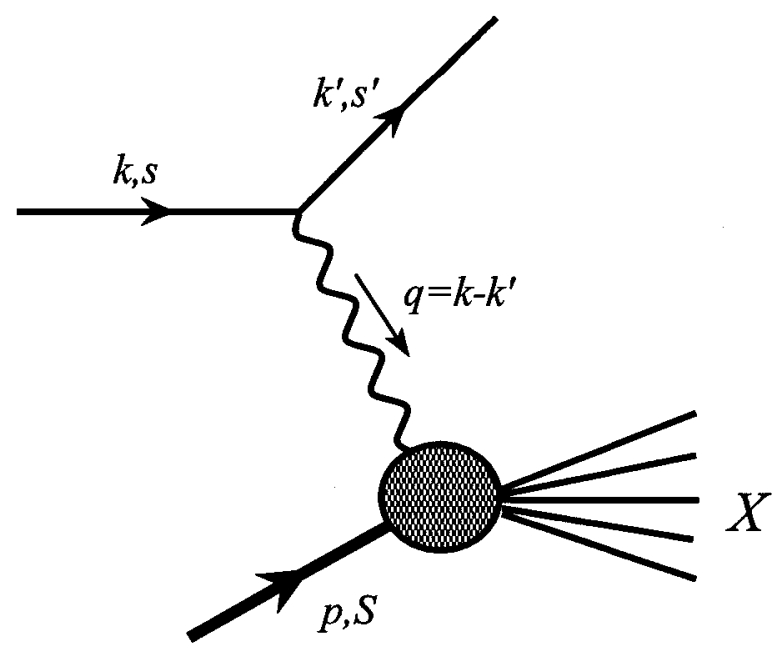

FIG. 1. First order Feynman diagram for lepton nucleon scattering.

\subsubsection{Formalism in One Photon Exchange}

Consider the inelastic scattering of leptons on nucleons. The double differential cross section, $\frac{d^{2} \sigma}{d E^{\prime} d \Omega}$, for detecting the final lepton in the solid angle $d \Omega$ can be expressed in tensor form as [9],

$$
\frac{d^{2} \sigma}{d \Omega d E^{\prime}}=\frac{\alpha^{2}}{2 M q^{4}} \frac{E^{\prime}}{E} L_{\mu \nu} W^{\mu \nu},
$$

where $\alpha$ is the fine structure constant, $L_{\mu \nu}$ is the leptonic tensor which describes the emission of the virtual photon, and $W^{\mu \nu}$ is the hadronic tensor which describes the absorption of the virtual photon by the nucleon. The leptonic tensor can be written as a sum of a symmetric $(S m)$ and an antisymmetric $(A)$ part [9],

$$
L_{\mu \nu}\left(k, s ; k^{\prime} s^{\prime}\right)=L_{\mu \nu}^{S m}\left(k ; k^{\prime}\right)+i L_{\mu \nu}^{A}\left(k, s ; k^{\prime}\right)+L_{\mu \nu}^{\prime S m}\left(k, s ; k^{\prime} s^{\prime}\right)+i L_{\mu \nu}^{A}\left(k ; k^{\prime} s^{\prime}\right) .
$$

On summing over the final lepton spin four vector $s^{\prime}$ we obtain,

$$
L_{\mu \nu}=2 L_{\mu \nu}^{S m}+2 i L_{\mu \nu}^{A}
$$

where,

$$
L_{\mu \nu}^{S m}\left(k ; k^{\prime}\right)=k_{\mu} k_{\nu}^{\prime}+k_{\mu}^{\prime} k_{\nu}-g_{\mu \nu}\left(k \cdot k^{\prime}-m^{2}\right)
$$

and

$$
L_{\mu \nu}^{A}\left(k, s ; k^{\prime}\right)=m \epsilon_{\mu \nu \alpha \beta} s^{\alpha}\left(k-k^{\prime}\right)^{\beta} .
$$

$g_{\mu \nu}=(1,-1-1-1)$ is the metric tensor and $\epsilon_{\mu \nu \alpha \beta}$ is the antisymmetric tensor. 
Similarly the hadronic tensor $W_{\mu \nu}$ also can be split into a symmetric and an antisymmetric part [9],

$$
W_{\mu \nu}(q ; p, S)=W_{\mu \nu}^{S m}(q ; p)+i W_{\mu \nu}^{A}(q ; p, S)
$$

where,

$$
\begin{aligned}
& \frac{1}{2 M} W_{\mu \nu}^{S m}(q ; p)=\left(-g_{\mu \nu}+\frac{q_{\mu} q_{\nu}}{q^{2}}\right) W_{1}\left(p \cdot q, q^{2}\right) \\
& +\left[\left(p_{\mu}-\frac{p \cdot q}{q^{2}} q_{\mu}\right)\left(\left(p_{\nu}-\frac{p \cdot q}{q^{2}} q_{\nu}\right)\right] \frac{W_{2}\left(p \cdot q, q^{2}\right)}{M^{2}}\right.
\end{aligned}
$$

and

$$
\begin{aligned}
& \frac{1}{2 M} W_{\mu \nu}^{A}(q ; p, S)= \\
& \epsilon_{\mu \nu \alpha \beta} q_{\alpha}\left[M S^{\beta} G_{1}\left(p \cdot q, q^{2}\right)+\left((p \cdot q) S^{\beta}-(S \cdot q) p^{\beta}\right) \frac{G_{2}\left(p \cdot q, q^{2}\right)}{M}\right] .
\end{aligned}
$$

Here, the coefficients $W_{1}, W_{2}$ are the unpolarized structure functions and $G_{1}$ and $G_{2}$ are the polarized structure functions. Structure functions describe the physics of the photon-nucleon interaction and are measurable quantities. In general the structure functions $W_{1}, W_{2}, G_{1}$ and $G_{2}$ depend on $\nu$ and $Q^{2}$. However, for scattering from point-like constituents inside the nucleon, i.e., quarks, the variable $x$ is more relevant than $\nu$. In the infinite momentum frame, $x$ is equal to the fraction of momentum carried by the struck quark. Therefore it is traditional to define new unpolarized and polarized structure functions, which depend on $x$ and $Q^{2}$ [9], [10]:

$$
\begin{aligned}
& M W_{1}\left(\nu, Q^{2}\right)=F_{1}\left(x, Q^{2}\right), \\
& \nu W_{2}\left(\nu, Q^{2}\right)=F_{2}\left(x, Q^{2}\right), \\
& M^{2} \nu G_{1}\left(\nu, Q^{2}\right)=g_{1}\left(x, Q^{2}\right), \\
& M \nu^{2} G_{2}\left(\nu, Q^{2}\right)=g_{2}\left(x, Q^{2}\right) .
\end{aligned}
$$

The contraction of the symmetric parts of $W_{\mu \nu}$ and $L_{\mu \nu}$ gives the spin averaged cross section,

$$
\begin{aligned}
\frac{d^{2} \sigma^{u n p}}{d \Omega d E^{\prime}} & =\frac{\alpha^{2}}{M Q^{4}} \frac{E^{\prime}}{E} L_{\mu \nu}^{(S m)} W^{\mu \nu(S m)} \\
& =\frac{4 \alpha^{2} E^{\prime 2}}{M Q^{4}}\left[2 \sin ^{2} \frac{\theta}{2} F_{1}\left(x, Q^{2}\right)+\frac{M}{\nu} \cos ^{2} \frac{\theta}{2} F_{2}\left(x, Q^{2}\right)\right]
\end{aligned}
$$


By contracting the antisymmetric part and taking cross section differences we can single out the spin dependent structure functions. In the case where the nucleon target and lepton beam are longitudinally polarized we obtain [9],

$$
\frac{d^{2} \sigma^{\downarrow \uparrow}}{d \Omega d E^{\prime}}-\frac{d^{2} \sigma^{\uparrow \uparrow}}{d \Omega d E^{\prime}}=\frac{4 \alpha^{2} E^{\prime}}{Q^{2} M E \nu}\left[\left(E+E^{\prime} \cos \theta\right) g_{1}\left(x, Q^{2}\right)-2 x M g_{2}\left(x, Q^{2}\right)\right] .
$$

The first arrow indicates the lepton beam helicity and the second arrow the spin direction of the target. Similarly for target nucleons that are transversely polarized with respect to the lepton beam we obtain [9],

$$
\frac{d^{2} \sigma^{\uparrow \hookleftarrow}}{d \Omega d E^{\prime}}-\frac{d^{2} \sigma^{\downarrow \uparrow}}{d \Omega d E^{\prime}}=\frac{4 \alpha^{2} E^{\prime 2}}{Q^{2} E M \nu} \sin \theta\left[g_{1}\left(x, Q^{2}\right)+\frac{4 x M E}{Q^{2}} g_{2}\left(x, Q^{2}\right)\right] .
$$

From these cross sections it is possible to construct longitudinal and transverse asymmetries,

$$
\begin{aligned}
& A_{\|}=\frac{\frac{d^{2} \sigma^{\downarrow \uparrow}}{d \Omega d E^{\prime}}-\frac{d^{2} \sigma^{\uparrow \uparrow}}{d \Omega d E^{\prime}}}{\frac{d^{2} \sigma^{\dagger \uparrow}}{d \Omega d E^{\prime}}+\frac{d^{2} \sigma^{\uparrow \uparrow}}{d \Omega d E^{\prime}}}, \\
& A_{\perp}=\frac{\frac{d^{2} \sigma^{\uparrow \leftarrow}}{d \Omega d E^{\prime}}-\frac{d^{2} \sigma^{\downarrow} \downarrow}{d \Omega d E^{\prime}}}{\frac{d^{2} \sigma^{\uparrow}}{d \Omega d E^{\prime}}+\frac{d^{2} \sigma^{\downarrow} \downarrow}{d \Omega d E^{\prime}}} .
\end{aligned}
$$

\subsubsection{Virtual Photon Asymmetries}

It is common practice and sometimes convenient to express structure functions and asymmetries $A_{\|}$and $A_{\perp}$ in terms of virtual photon asymmetries which are defined using photoabsorption cross sections. The Feynman diagram given in Fig. 1 can be viewed as a scattering process consisting of two parts: the emission of a virtual photon by the incident lepton and the absorption of the same photon by the nucleon. Using the optical theorem, the absorption cross section of the photon by the nucleon can be expressed as the imaginary part of the forward virtual photon-nucleon Compton scattering amplitude. The forward Compton scattering amplitude can be expanded in helicity amplitudes. For forward Compton scattering the helicity amplitude $\mathcal{M}_{i j, i^{\prime} j^{\prime}}$ can be computed in terms of the hadronic tensor $W_{\mu \nu}[11]$,

$$
\mathcal{M}_{i, j ; i^{\prime}, j^{\prime}}=\epsilon_{i}^{\mu^{*}} \epsilon_{i^{\prime}}^{\nu} W_{\mu \nu}
$$

where $\epsilon^{\mu}$ is the polarization vector of the virtual photon and $i, j, i^{\prime}, j^{\prime}$ are the spin projections of the incident virtual photon, incident nucleon, scattered virtual photon and scattered nucleon. Between a virtual photon with 3 possible polarization states 
and a spin $1 / 2$ target with 2 polarization states, it is possible to make 10 combinations of angular momentum conserving amplitudes. However, due to the invariance of parity,

$$
\mathcal{M}_{i, j ; i^{\prime}, j^{\prime}}=\mathcal{M}_{-i,-j ;-i^{\prime},-j^{\prime}}
$$

and invariance of time reversal,

$$
\mathcal{M}_{i, j ; i^{\prime}, j^{\prime}}=\mathcal{M}_{i^{\prime}, j^{\prime} ; i, j}
$$

the 10 amplitudes reduce to four independent helicity amplitudes,

$$
\mathcal{M}_{1,-\frac{1}{2} ; 1,-\frac{1}{2}}, \mathcal{M}_{1, \frac{1}{2} ; 1, \frac{1}{2}}, \mathcal{M}_{0, \frac{1}{2} ; 0, \frac{1}{2}}, \mathcal{M}_{1,-\frac{1}{2} ; 0, \frac{1}{2}}
$$

By substituting the expression for the hadronic tensor (equation (6)) into equation (18) and applying the optical theorem we obtain [11],

$$
\begin{aligned}
& \sigma_{1 / 2}^{T}=\frac{4 \pi \alpha^{2}}{K} \mathcal{M}_{1, \frac{1}{2} ; 1, \frac{1}{2}}=\frac{4 \pi^{2} \alpha}{M K}\left(F_{1}+g_{1}-\frac{2 M x}{\nu} g_{2}\right), \\
& \sigma_{3 / 2}^{T}=\frac{4 \pi \alpha^{2}}{K} \mathcal{M}_{1,-\frac{1}{2} ; 1,-\frac{1}{2}}=\frac{4 \pi^{2} \alpha}{M K}\left(F_{1}-g_{1}+\frac{2 M x}{\nu} g_{2}\right), \\
& \sigma_{1 / 2}^{L}=\frac{4 \pi \alpha^{2}}{K} \mathcal{M}_{0, \frac{1}{2} ; 0, \frac{1}{2}}=\frac{4 \pi^{2} \alpha}{K}\left[\frac{F_{2}}{\nu}\left(1+\frac{\nu_{2}}{Q^{2}}\right)-\frac{1}{M} F_{1}\right], \\
& \sigma_{1 / 2}^{T L}=\frac{4 \pi \alpha^{2}}{K} \mathcal{M}_{0, \frac{1}{2} ; 0,-\frac{1}{2}}=\frac{4 \pi^{2} \alpha}{K} \frac{\sqrt{Q^{2}}}{M \nu}\left(g_{1}+g_{2}\right),
\end{aligned}
$$

where $K=\frac{W^{2}-M^{2}}{2 M}$. The virtual photon asymmetries $A_{1}$ and $A_{2}$ are defined as,

$$
\begin{gathered}
A_{1}\left(x, Q^{2}\right)=\frac{\sigma_{1 / 2}^{T}-\sigma_{3 / 2}^{T}}{\sigma_{1 / 2}^{T}+\sigma_{3 / 2}^{T}}=\frac{g_{1}\left(x, Q^{2}\right)-\frac{Q^{2}}{\nu^{2}} g_{2}\left(x, Q^{2}\right)}{F_{1}\left(x, Q^{2}\right)}, \\
A_{2}\left(x, Q^{2}\right)=\frac{2 \sigma_{1 / 2}^{T L}}{\sigma_{1 / 2}^{T}+\sigma_{3 / 2}^{T}}=\frac{g_{1}\left(x, Q^{2}\right)+g_{2}\left(x, Q^{2}\right)}{F_{1}\left(x, Q^{2}\right)} \frac{1}{\nu / \sqrt{Q^{2}}} .
\end{gathered}
$$

$A_{1}$ and $A_{2}$ can be related to experimental asymmetries $A_{\|}$and $A_{\perp}$ introduced in section II.2 by,

$$
\begin{aligned}
& A_{\|}=D\left(A_{1}+\eta A_{2}\right), \\
& A_{\perp}=d\left(A_{2}-\zeta A_{1}\right),
\end{aligned}
$$

where $D, \eta, d$ and $\zeta$ are kinematic variables defined as,

$$
D=\frac{1-\epsilon \frac{E^{\prime}}{E}}{1+\epsilon R}
$$




$$
\begin{gathered}
\eta=\frac{\epsilon \frac{\sqrt{Q^{2}}}{E}}{1-\epsilon \frac{E^{\prime}}{E}} \\
\zeta=\frac{1+\epsilon}{2 \epsilon} \eta
\end{gathered}
$$

where,

$$
\epsilon=\frac{1}{1+2 \tan ^{2} \frac{\theta}{2}\left(1+\frac{\nu^{2}}{Q^{2}}\right)}
$$

and

$$
R=\frac{2 \sigma_{1 / 2}^{L}}{\sigma_{1 / 2}^{T}+\sigma_{3 / 2}^{T}} .
$$

The factor $D$ is known as the depolarization factor and it accounts for the loss of polarization from the lepton to the virtual photon. The ratio $R$ is related to the unpolarized structure functions $F_{1}$ and $F_{2}$ by the relation [12],

$$
R=\frac{F_{2}}{2 x F_{1}}\left(1+\frac{4 M^{2} x^{2}}{Q^{2}}\right)-1
$$

Separation of $A_{1}$ and $A_{2}$ allows for an independent measurement of both spin structure functions $g_{1}$ and $g_{2}$ given that the unpolarized structure functions $F_{1}$ and $R$ are known. $A_{1}$ and $A_{2}$ can be separated by either measuring both $A_{\|}$and $A_{\perp}$ at fixed kinematics or by measuring $A_{\|}$at different beam energies at fixed $Q^{2}$ and $W$. The latter method is possible due to the fact that kinematic factors in front of $A_{1}$ and $A_{2}$ are different for different beam energies.

\subsection{SPIN STRUCTURE FUNCTIONS}

It is well known that hadrons are not fundamental particles but are composite objects of point like dirac particles called quarks, which interact with each other through the strong force. The gauge boson of the strong interaction is the gluon.

The structure we observe by probing the nucleon by the virtual photon depends on the virtuality or $Q^{2}$ of the photon. At very large $Q^{2}$ the distances probed by the virtual photon are small and the parton structure of the nucleon can be observed. This region is known as the deep inelastic scattering region (Fig. 2). As $Q^{2} \longrightarrow \infty$, the effective coupling constant for the strong interaction, $\alpha_{s} \longrightarrow 0$. This is known as asymptotic freedom, where quarks behave like free particles. The naive parton model interpretations of structure functions are based on this assumption. At finite but large $Q^{2}$ the strong coupling constant no longer can be treated as zero. In this 


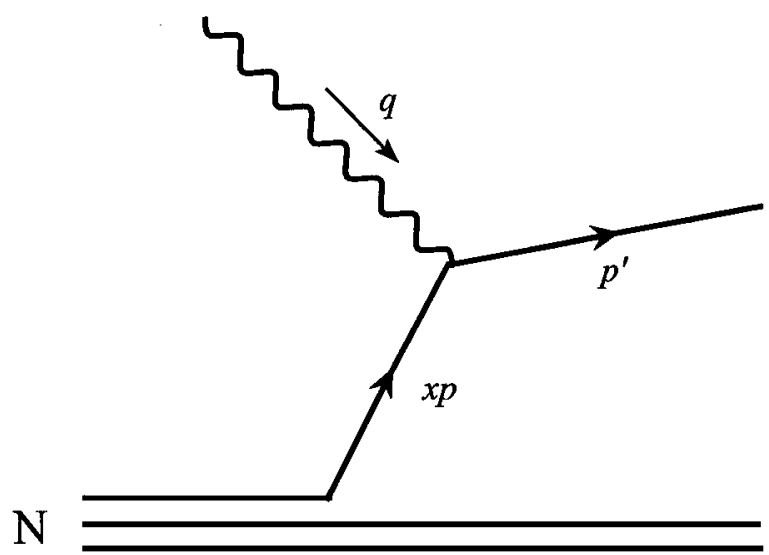

FIG. 2. Parton model description of deep inelastic electron nucleon scattering. $x p$ and $p^{\prime}$ are initial and final momentum of the quark.

region the theory of strong interaction Quantum Chromo Dynamics (QCD) can be treated perturbatively.

At very low $Q^{2}$ the wavelength of the virtual photon is not small enough to probe the individual quark composition of the nucleon; instead it probes the nucleon as an extended coherent object. This region is dominated by excitation of baryon resonances and (multi-)pion continuum states.

In this section, the $Q^{2}$ dependence of spin structure functions $g_{1}$ and $g_{2}$ will be discussed.

\subsubsection{Parton Model Interpretation of Structure Functions}

The parton model, which is described in a frame where the nucleon has an infinite momentum, treats partons (quarks and gluons) as free non-interacting constituents each carrying a fraction $x$ of the nucleon four-momentum and traveling in the same direction as the parent hadron. Hence the lepton-nucleon cross-section is described as the incoherent sum over the contribution of all lepton-parton cross sections. The parton model then relates the structure functions to the quark distribution functions according to,

$$
\begin{aligned}
& F_{1}(x)=\frac{1}{2} \sum_{i} e_{i}^{2} q_{i}(x), \\
& F_{2}(x)=x \sum_{i} e_{i}^{2} q_{i}(x)=2 x F_{1}(x), \\
& g_{1}(x)=\frac{1}{2} \sum_{i} e_{i}^{2} \Delta q_{i}(x),
\end{aligned}
$$




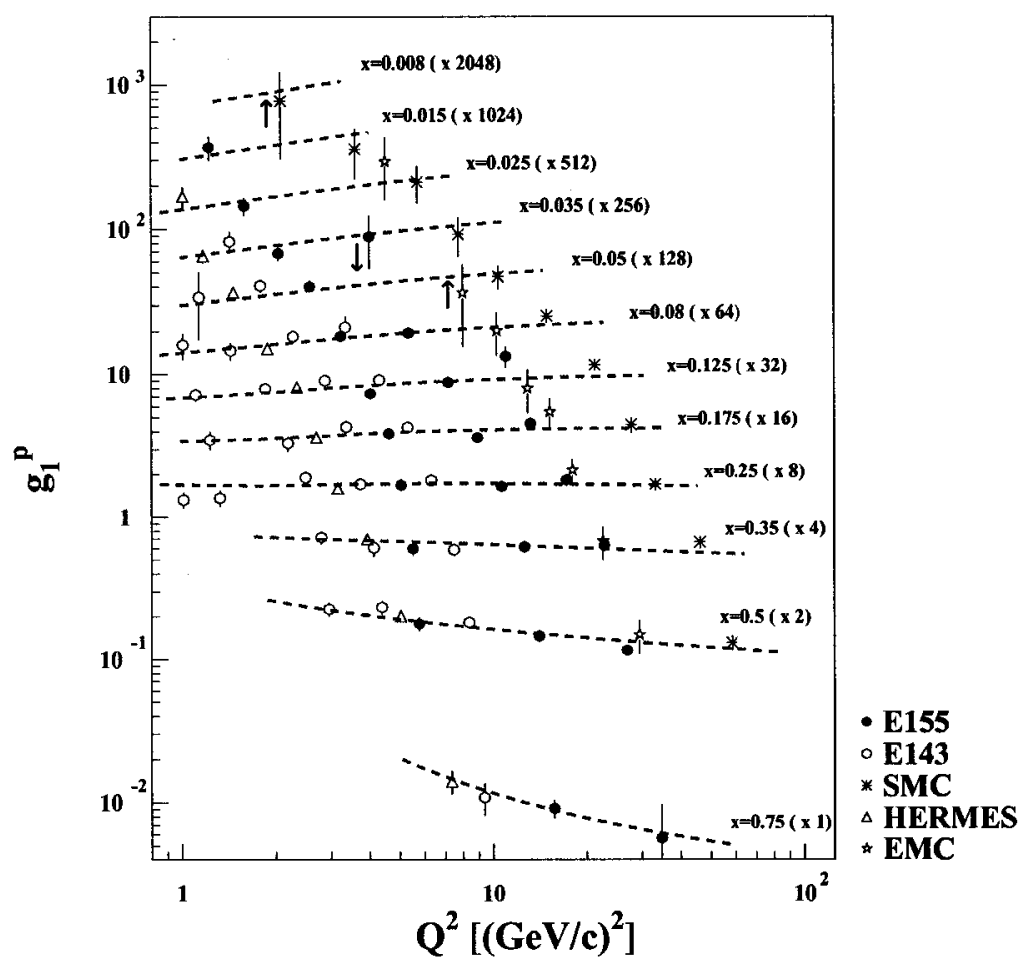

FIG. 3. Deep inelastic scattering world data on $g_{1}^{p}$ [13] as of 1999. The figure shows the $Q^{2}$ dependence of $g_{1}^{p}$ for different $x$ vales. Dashed lines are a next to leading order QCD fit.

$$
g_{2}(x)=0
$$

Here $q_{i}(x)$ gives the probability to find a quark of flavor $i$ in the nucleon with momentum fraction $x$ and charge $e_{i}$ and $\Delta q_{i}(x)=q_{i}^{\uparrow}(x)-q_{i}^{\downarrow}(x)$, where $q_{i}^{\uparrow}(x)$ is the quark distribution of $i^{\text {th }}$ flavor with the helicity parallel to the nucleon helicity and $q_{i}^{\downarrow}(x)$ is the quark distribution of $i^{\text {th }}$ flavor with the helicity antiparallel to the nucleon helicity. The most important feature of the parton model results is that the structure functions are independent of $Q^{2}$ and depend only on $x$.

\subsection{2 $Q^{2}$ Dependence of the Spin Structure Function $g_{1}$}

\section{$Q^{2}$ evolution of $g_{1}$ in pQCD}

The naive parton model discussed in the previous section makes the assumption that the partons in the nucleus do not interact with each other and hence structure functions are only a function of $x$. However, structure functions show a significant $Q^{2}$ dependence (Fig. 3) due to gluon bremsstrahlung and scattering from quark 
(a)

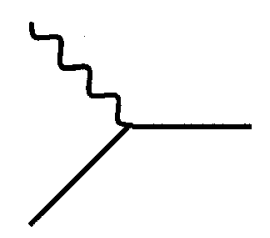

(b)

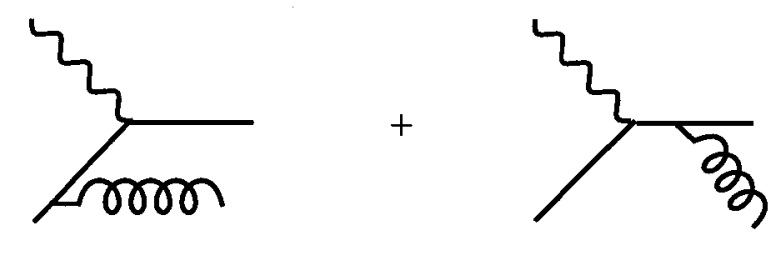

(c)
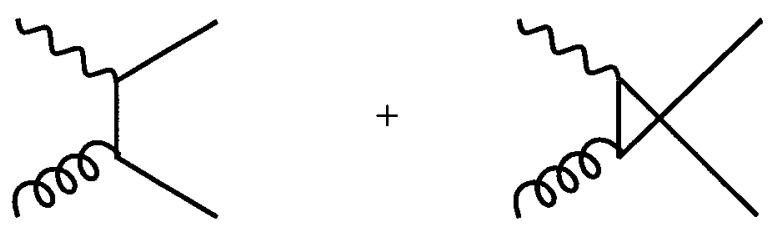

FIG. 4. Leading order and next to leading order contributions to electron scattering from a quark. (a) Leading order term (b) Gluon bremsstrahlung. These two graphs interfere. (c) A gluon producing a quark anti-quark pair and then one couples to the virtual photon. These two graphs interfere.

antiquark pairs produced by gluons (Fig. 4). At finite $Q^{2}$ Bjorken scaling is broken and the parton distribution functions show a slow logarithmic $Q^{2}$ dependence. These higher order QCD terms can be computed in perturbative QCD (pQCD) [14] and will be the focus of this section. The $Q^{2}$ dependence of the spin structure function $g_{1}$ at next to leading order (NLO) in pQCD is given by [14],

$$
\begin{aligned}
g_{1}\left(x, Q^{2}\right)= & \frac{1}{2} \sum_{i} e_{i}^{2} C_{q}\left(x, \alpha_{s}\right) \bigotimes \Delta q_{i}\left(x, Q^{2}\right) \\
& +C_{g}\left(x, \alpha_{s}\right) \bigotimes \Delta G\left(x, Q^{2}\right),
\end{aligned}
$$

where the sum is taken over all active quark flavors $N_{f}$. The convolution $\otimes$ is defined as,

$$
f\left(x, \alpha_{s}\right) \bigotimes g\left(x, Q^{2}\right)=\int_{x}^{1} \frac{d y}{y} f\left(\frac{x}{y}, \alpha_{s}\right) g\left(x, Q^{2}\right),
$$

$C_{q}\left(x, \alpha_{s}\right)$ and $C_{g}\left(x, \alpha_{s}\right)$ are Wilson coefficients and correspond to the photon-quark and photon-gluon hard scattering cross section respectively. These coefficients can be expanded in powers of $\alpha_{s}$,

$$
C_{i}\left(x, \alpha_{s}\right)=C_{i}^{0}(x)+\frac{\alpha_{s}}{2 \pi}\left(Q^{2}\right) C_{i}^{(1)}+\ldots \ldots
$$


In the leading order $C_{q}^{o}(x)=\delta(1-x)$ and $C_{g}^{0}(x)=0$. The $Q^{2}$ evolution of the quark $(\Delta q)$ and gluon $(\Delta G)$ distribution functions is described by the Dokshitzer-GribovLipatov-Altarelli-Parisi (DGLAP) [15] equations [14],

$$
\begin{aligned}
& \frac{d}{d \ln Q^{2}} \Delta q_{N S}\left(x, Q^{2}\right)=\frac{\alpha_{s}\left(Q^{2}\right)}{2 \pi} P_{q q}^{N S} \otimes \Delta q_{N S}, \\
& \frac{d}{d \ln Q^{2}}\left(\begin{array}{c}
\Delta q_{S} \\
\Delta G
\end{array}\right)=\frac{\alpha_{s}\left(Q^{2}\right)}{2 \pi}\left(\begin{array}{cc}
P_{q q} & P_{q G} \\
P_{G q} & P_{G G}
\end{array}\right) \otimes\left(\begin{array}{c}
\Delta q_{S} \\
\Delta G
\end{array}\right) .
\end{aligned}
$$

For three quark flavors, the non-singlet quark distribution $\Delta q_{N S}\left(x, Q^{2}\right)$ is given by,

$$
\Delta q_{N S}\left(x, Q^{2}\right)=(\Delta u+\Delta \bar{u})-\frac{1}{2}(\Delta d+\Delta \bar{d})-\frac{1}{2}(\Delta s+\Delta \bar{s})
$$

and the singlet quark distribution $\Delta q_{S}\left(x, Q^{2}\right)$ is given by,

$$
\Delta q_{S}\left(x, Q^{2}\right)=(\Delta u+\Delta \bar{u})+(\Delta d+\Delta \bar{d})+(\Delta s+\Delta \bar{s})
$$

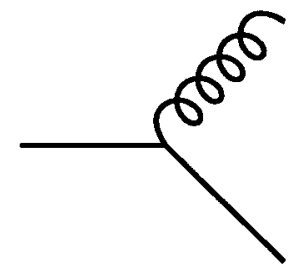

(a)

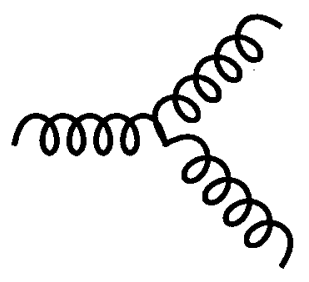

(b)

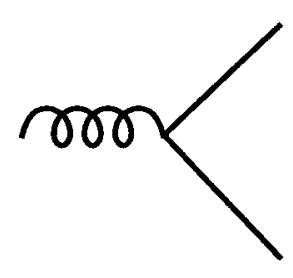

(c)

FIG. 5. Vertices which determine the splitting functions [16](a) $P_{q q}$ and $P_{q G}$ (b) $P_{G G}$ and $(\mathrm{c}) P_{G q}(g \rightarrow q \bar{q})$

The splitting functions $P_{i j}$ (Fig. 5) can be expanded as series in the strong coupling constant,

$$
\Delta P_{i j}=\Delta P_{i j}^{(0)}+\frac{\alpha_{s}\left(Q^{2}\right)}{2 \pi} \Delta P_{i j}^{(1)}+\ldots \ldots
$$

$\Delta P_{q q}$ is proportional to the probability of quark splitting into a quark and a gluon. $\Delta P_{G q}$ considers the possibility of a gluon producing a quark antiquark pair. Together with $\Delta P_{G G}$ it determines the evolution of the gluon density in the nucleon. Calculation of Wilson coefficients and splitting functions at and beyond NLO depends on the renormalization scheme used. Both of these quantities for the polarized case have been calculated in NLO in the Modified-Minimal-subtraction (M $\overline{\mathrm{S}}$ ) scheme [17]. 
If the quark and gluon distribution functions are known for a particular $Q^{2}$ for the complete $x$ range then they can be calculated for other $Q^{2}$ values using DGLAP equations.

\section{Higher twist corrections}
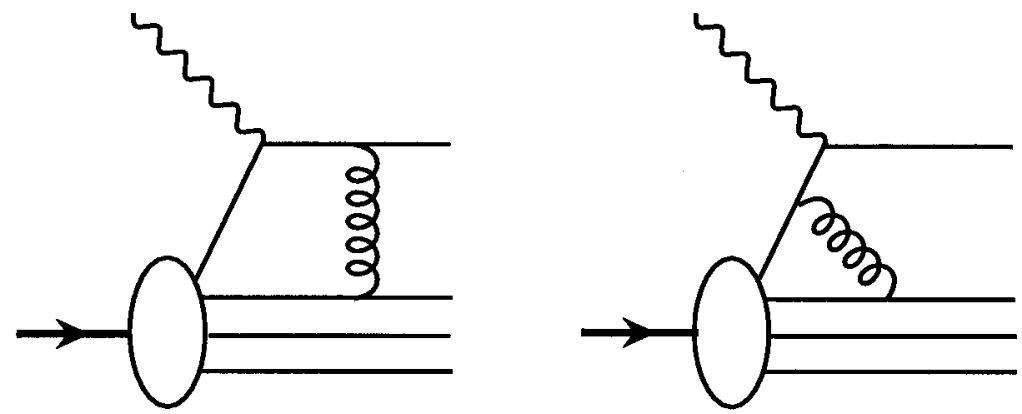

FIG. 6. Feynman diagrams that contribute to higher twist effects.

In addition to the logarithmic scaling violations that were discussed in the previous section, additional corrections, which arise due to multi-parton correlations in the nucleon (Fig. 6), known as higher twists, have to be taken into account. These corrections, which are relatively small at large $Q^{2}$ are expected to be non-negligible in the low $Q^{2}$, region. In general, contributions to structure functions that are suppressed relative to leading order contributions by the powers of $Q^{2}$ are known as higher twist corrections. The Operator Product Expansion (OPE) [18] is generally used as a theoretical tool to describe these corrections. For inclusive inelastic scattering OPE allows one to write composite operators as a sum of finite local operators multiplied by singular coefficient functions [10],

$$
J(x) J(0) \sim \sum_{\tau, n} C_{\tau, n}\left(x^{2}\right) x_{\mu_{1} \ldots \ldots \ldots .} x_{\mu_{n}} O_{\mu_{1} \ldots \ldots \ldots \mu_{n}}^{\tau}(0)
$$

where the twist is given by $\tau$ and $n$ is the spin of the operator $O_{i}^{\tau}$. The coefficients $C_{\tau, n}$, which describe the photon-quark interactions, are the Wilson coefficients and are calculable in perturbative QCD. The long distance structure of the nucleon is contained in the matrix elements of the operators $O_{\mu_{1} \ldots \ldots \ldots \mu_{n}}^{\tau}$. OPE allows one to expand the Cornwall-Norton moments of a scaling function $F\left(x, Q^{2}\right)$ [19],

$$
M_{n}\left(Q^{2}\right)=\int_{0}^{1} d x x^{n-2} F\left(x, Q^{2}\right),
$$


in powers of $1 / Q^{2}$,

$$
M_{n}\left(Q^{2}\right)=\sum_{k=0}^{\infty} E_{n k}\left(Q^{2} / \mu^{2}\right) M_{n k}\left(\mu^{2}\right)\left(\frac{1}{Q^{2}}\right)^{k},
$$

where $\mu$ is a normalization scale. The coefficients $E_{n k}$ are calculable in pQCD and $M_{n k}\left(\mu^{2}\right)$ are nucleon matrix elements of local operators which describe the quark gluon structure of the nucleon. In general the terms in equation (50), except for the first term, are known as higher twist corrections. The corresponding corrections for the structure functions themselves can be obtained by taking the inverse Mellin transforms of moments [10],

$$
F\left(x, Q^{2}\right)=\frac{1}{2 \pi i} \int_{a-i \infty}^{a+i \infty} d n x^{1-n} M_{n}\left(Q^{2}\right) .
$$

The value $a$ is chosen such that the contour of integration lies to the right of all singularities of $M_{n}$ in the complex $n$ plane. The use of OPE in particular for the first moment of $g_{1}$ will be discussed in more detail in section 2.5.2.

\subsubsection{Resonance Contribution to $g_{1}$}

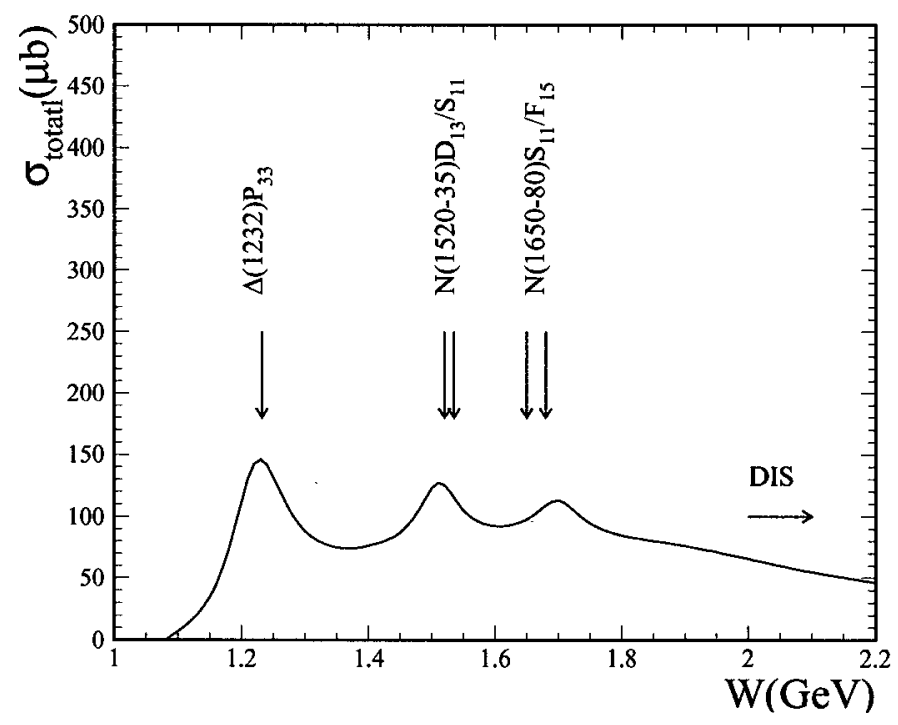

FIG. 7. Cross section for the inclusive reaction $e p \longrightarrow e^{\prime} X$ at $Q^{2}=1.4 \mathrm{GeV}^{2}$. Some of the well known resonances are indicated by arrows.

Resonances are spin or momentum excited states of the nucleon. These excited states have short life times and decay mainly by emitting a meson. The mass region below $W=2 \mathrm{GeV}$ is traditionally known as the resonance region. A resonance 
occurs at an invariant mass $W_{\text {res }}$ when a nucleon absorbs a photon of energy $\nu^{\text {res }}=$ $\frac{W_{r e s}^{2}-M^{2}+Q^{2}}{2 M}$. In inclusive measurements most of the resonances are hidden under three major resonance bumps making it difficult to separate and identify different excited states. The structure of different resonances depends on individual characteristics of the hadronic bound states. Electroproduction of resonances are often described using multipoles $E_{l \pm}, M_{l \pm}$ and $S_{l \pm}$, which characterize the excitation mechanism, $E$ electric, $M$-magnetic and $S$-coulomb or scalar, and the orbital $(l)$ and total angular momentum of the transition. In general the standard notation for identifying a resonance is $l_{2 I 2 J}$, where $l=0(S), 1(P), 2(D), 3(F)$ is the orbital angular momentum, $I=1 / 2$ or $3 / 2$ is the isospin and $J=|l \pm 1 / 2|$ is the total angular momentum of the final meson-baryon state. Fig. 7 shows some of the well known resonances for the inclusive reaction $e p \longrightarrow e^{\prime} X$. The entire excitation spectrum includes a nonresonant background in addition to resonances. The first resonance is the well known $\Delta(1232)$ resonance. In the $\mathrm{SU}(6)$ symmetric quark model, the transition from the spin $1 / 2$ nucleon ground state to the spin $3 / 2 \Delta(1232)$ excited state occurs dominantly due to a quark spin flip which corresponds to a $M_{1+}$ magnetic dipole transition and $E_{1+}=S_{1+}=0$. For a pure $M_{1+}$ transition the virtual photon asymmetry $A_{1}$ for the $\Delta(1232)$ excitation is -0.5 . However, non-zero values of the electric $\left(E_{1+}\right)$ and scaler $\left(S_{1+}\right)$ quadrupole transition amplitudes have been observed (Fig. 8) in several Jefferson Lab experiments [20]. The $\Delta(1232)$ resonance is expected to play a dominant role in the moments of spin structure functions at low $Q^{2}$ values.

In order to separate and identify different excited states, the resonance decay products have to be measured. The observables of the exclusive process $e+N \longrightarrow$ $e^{\prime}+R$ are usually described in terms of helicity amplitudes [21],

$$
G_{m}=\frac{1}{2 M}\left\langle R, \lambda^{\prime}=m-\frac{1}{2}\left|\epsilon_{\mu}^{(m)} \cdot j^{\mu}(0)\right| N, \lambda=\frac{1}{2}\right\rangle .
$$

$G_{m}$ describes the amplitude for producing a final baryon $R$ with helicity $\lambda^{\prime}$ by absorbing a virtual photon with polarization $m= \pm 1,0$ on a nucleon $N$ with helicity $\lambda$. The photon polarization vectors are,

$$
\begin{aligned}
\epsilon^{( \pm)} & =(0, \mp 1,-i, 0) / \sqrt{2}, \\
\epsilon^{(0)} & =\frac{1}{Q}(|\vec{q}|, 0,0, \nu) .
\end{aligned}
$$

The contribution of an isolated resonance to $g_{1}$ can be described in terms of helicity 


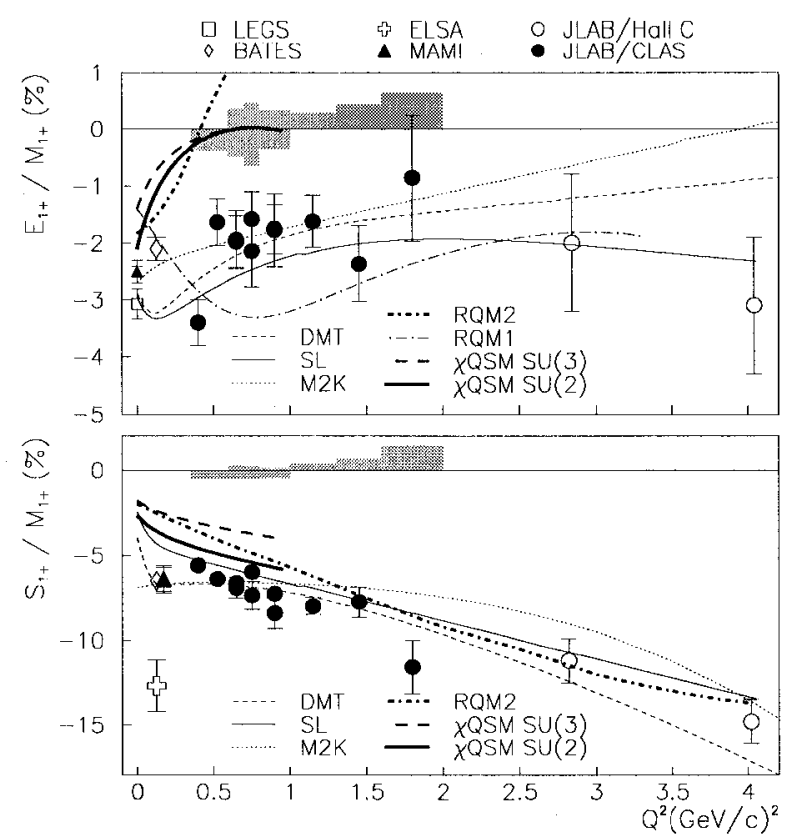

FIG. 8. The ratios $E_{1+} / M_{1+}, S_{1+} / M_{1+}$ for $\gamma^{*} p \Delta(1232)$ [20]. Plot includes MAMI, LEGS and BATES results taken after 1990 and recent Jefferson Lab results

amplitudes. For a given resonance $g_{1}$ can be written as,

$$
g_{1}=\frac{1}{1+Q^{2} / \nu^{2}} M^{2} \delta\left(W^{2}-M_{R}^{2}\right)\left[\left|G_{+}\right|^{2}-\left|G_{-}\right|^{2}+(-)^{S_{R}-\frac{1}{2}} \eta_{R} \frac{\sqrt{2 Q^{2}}}{\nu} G_{0}^{*} G_{+}\right]
$$

where $S_{R}$ and $\eta_{R}$ are the spin and parity of the resonances. The delta function is an approximation to the Breit-Wigner form,

$$
\delta\left(W^{2}-M\right)^{2} \approx \frac{1}{2 M_{R}} \frac{\Gamma_{R} / 2 \pi}{\left(W-M_{R}\right)^{2}+\Gamma_{R}^{2} / 4},
$$

where $M_{R}$ and $\Gamma_{R}$ are the mass and width of the resonance.

There has been some effort in incorporating the existing world data to parameterize $g_{1}^{p}$ in the resonance region [22]. These parameterizations have been performed by first expressing the contribution of each resonance to $g_{1}$ in terms of helicity amplitudes and then fitting the existing world data to obtain the $Q^{2}$ dependence of individual resonances. However the comparison of these models to proton data shows significant differences [23]. These models can further be developed when new data on the helicity amplitudes in the resonance region become available. 


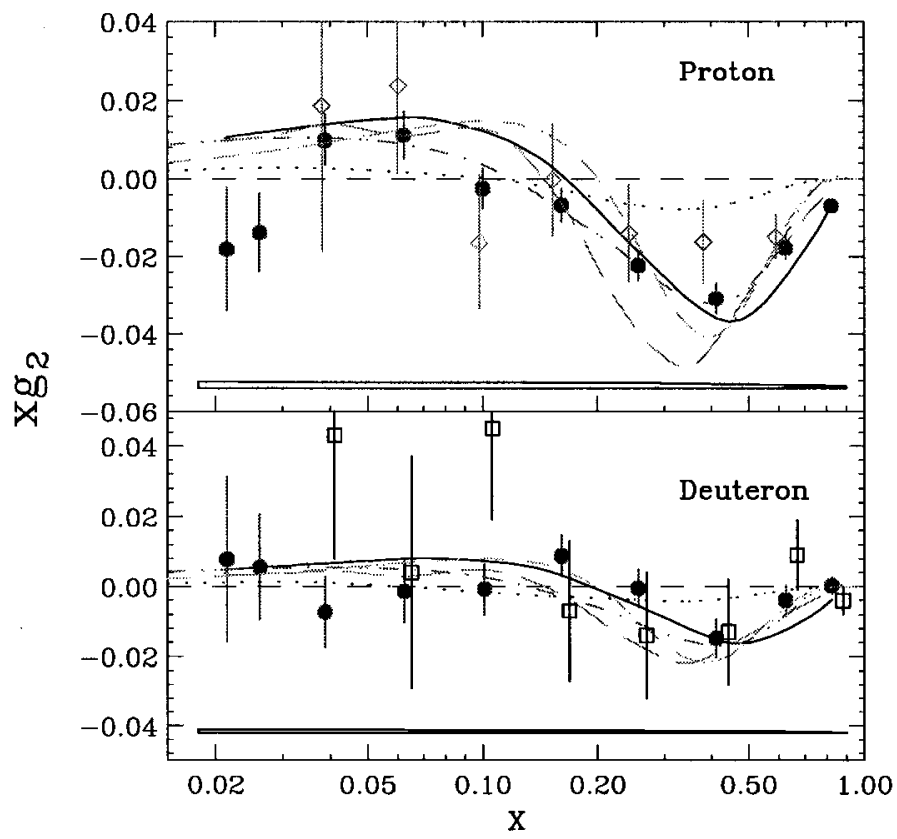

FIG. 9. Measurement of $x g_{2}^{p}$ and $x g_{2}^{d}$ [24]. Data are from E155 (solid circle and open diamond) and E143 (open square) experiments at SLAC. The solid curve shows the $g_{2}^{W W}$ calculations.

\subsubsection{Spin Structure Function $g_{2}$}

In the naive parton model, the nucleon transverse spin dependent structure function $g_{2}$ is predicted to be zero. However, the interpretation of $g_{2}$ in OPE indicates nonzero values. In general $g_{2}$ can be written as,

$$
g_{2}\left(x, Q^{2}\right)=g_{2}^{W W}\left(x, Q^{2}\right)+g_{2}^{H T}\left(x, Q^{2}\right)
$$

where $g_{2}^{W W}\left(x, Q^{2}\right)$, which contains only twist-2 terms coming from the same set of operators that contribute to $g_{1}$, is given by the Wandzura-Wilczek relation [25],

$$
g_{2}^{W W}\left(x, Q^{2}\right)=-g_{1}\left(x, Q^{2}\right)+\int_{x}^{1} \frac{g_{1}\left(y, Q^{2}\right)}{y} d y
$$

and the second term, $g_{2}^{H T}\left(x, Q^{2}\right)$, includes another twist-2 part, which arises due to quark transverse polarization, and a twist-3 part coming from quark-gluon interactions inside the nucleon (Fig. 9). 


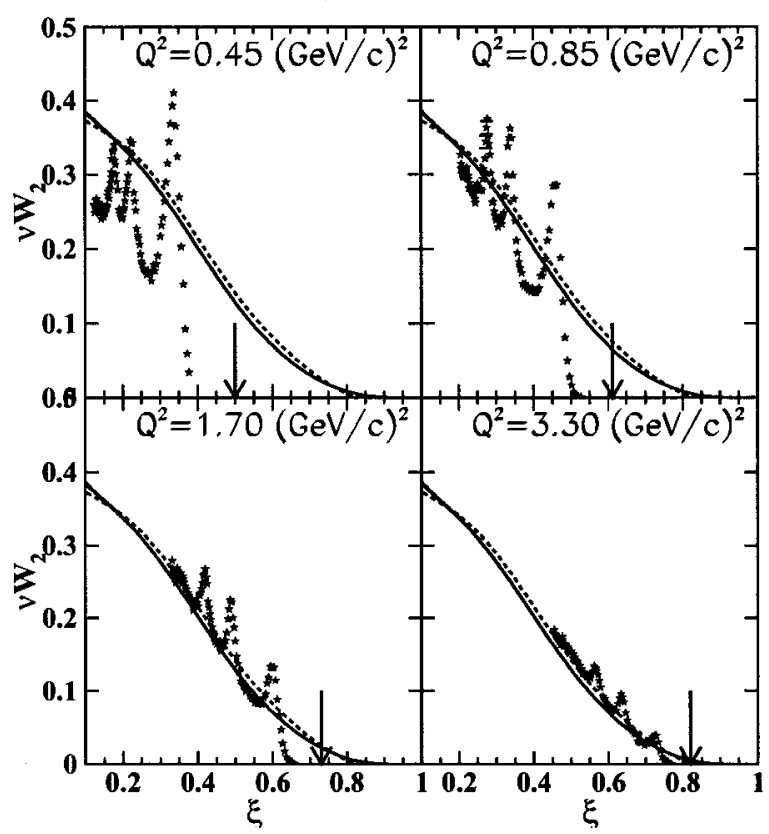

FIG. 10. The spin independent structure function $\nu W_{2}$ plotted as a function of $\xi$ for hydrogen at different $Q^{2}$ values [27]. The solid and dashed lines are a fit to deep inelastic structure function data at $Q^{2}=10\left(\mathrm{GeV} / \mathrm{c}^{2}\right)$ and $Q^{2}=5\left(\mathrm{GeV} / \mathrm{c}^{2}\right)$ respectively.

\subsection{QUARK-HADRON DUALITY}

The phenomenon known as quark-hadron duality was first observed by Bloom and Gilman [26] in 1970. Later this behavior was experimentally confirmed in the resonance region for the unpolarized structure functions [27] (Fig. 10). Quark hadron duality postulates that the electroproduction of resonances averages to the deep inelastic structure functions if a proper scaling variable that connects the two kinematic regions is used. In other words, it states that although electroproduction of resonances is a non-perturbative phenomenon if averaged over a large kinematic region, physical quantities calculated in the hadronic description give the same results as if they were calculated using the partonic description. Further duality may also hold for each resonance over restricted regions in $W$, which is known as local duality.

The interpretation of $x$ as the momentum fraction carried by the struck quark is only valid if $Q^{2}>>M^{2}$. However at low $Q^{2}$ a kinematic correction that arises due to the non-zero target mass has to be applied. Although this correction has a similar $1 / Q^{2}$ evolution as the twist- 4 corrections, it is not considered a higher twist effect. 
By using a proper scaling variable known as the Natchmann scaling variable,

$$
\xi=\frac{2 x}{1+\sqrt{1+4 x^{2} \frac{M_{N}^{2}}{Q^{2}}}},
$$

target mass effects are taken into account.

De Rujula, Georgi and Politzer [28] have pointed out that duality can be understood from an operator product expansion (OPE) of moments of structure functions. The OPE allows one to separate the short and long distance contributions to the moments of structure functions. Therefore equation (50) can be formally separated in to the leading twist term which can be calculated in pQCD and higher twist terms,

$$
M_{n}\left(Q^{2}\right)=\int_{0}^{1} \xi^{n-2} F\left(\xi, Q^{2}\right) d \xi=A_{n}\left(Q^{2}\right)+\sum_{k=1}^{\infty}\left[n \frac{M_{0}^{2}}{Q^{2}}\right]^{k} B_{n k}\left(Q^{2}\right),
$$

where the operators of twist $2 k+2$ gives the $k^{\text {th }}$ term in the sum and $M_{0}$ is a characteristic strong interaction mass which is of the order of a few hundred $\mathrm{MeV}$. The use of the variable $\xi$ ensures the inclusion of all target mass correction terms, which are powers of $M^{2} / Q^{2}$, in the leading twist, $A_{n}\left(Q^{2}\right)$, terms. Then the authors

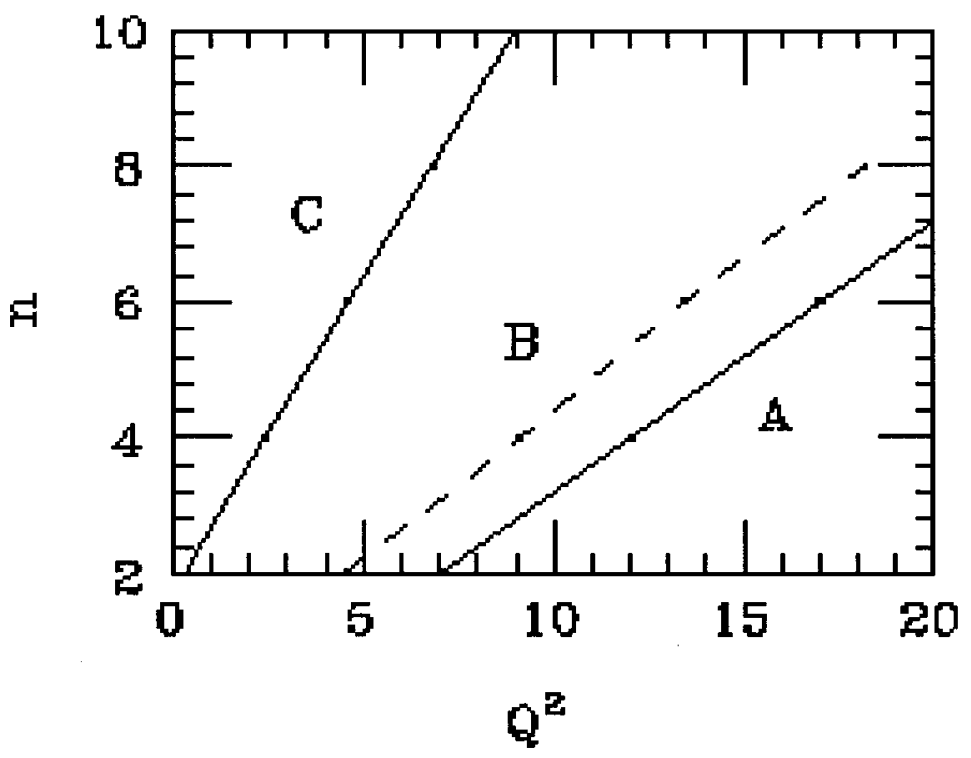

FIG. 11. Different kinematic regions where (A) higher twist effects are negligible, (B) higher twist effects are non-negligible however are small and $(\mathrm{C})$ higher twist effects are large and nonperturbative [19]. Details of each region are explained in the text.

argue that the $n-Q^{2}$ plane can be divided into three physically different regions 
(Fig. 11),

$$
\begin{aligned}
& A \longleftrightarrow n \ll Q^{2} / M_{0}^{2} \\
& B \longleftrightarrow n \leq Q^{2} / M_{0}^{2} \\
& C \longleftrightarrow n \geq Q^{2} / M_{0}^{2}
\end{aligned}
$$

Depending upon the moment, $n-2$, of the structure function $F$, a cutoff for $\mathrm{A}, \mathrm{B}$ and $\mathrm{C}$ in $Q^{2}$ can be identified depending on the importance of the higher twist contributions to that function (57). In region $A$ the effects of the higher twist operators, the second term in equation (57), are negligible and structure functions are smooth except for a small logarithmic $Q^{2}$ dependence. If $n \geq Q^{2} / M_{0}^{2}$, higher-twist dominates and the first term in (57) is much smaller than the second term. This is the region near or below the threshold. Region $\mathrm{B}$, where higher-twists are neither large nor negligible, can be identified as the resonance region, where bumps in $F\left(\xi, Q^{2}\right)$ at $W$ corresponding to resonances are seen. However, the moments of $F$ for these bumps cannot be much different than the corresponding scaling value since the higher twist effects are not large. Therefore, if $F$ rises above the scaling value it has to fall in the neighboring $\xi$ region in order to compensate for the increase in the moments above the scaling value. This behavior explains why resonances average to a smooth scaling curve.

Recently Carlson and Mukhopadhyay [21] have used a somewhat different approach in explaining duality for the spin structure function $g_{1}$. Using counting rules they obtain the following behavior for helicity amplitudes [given in equation (52)] at large $Q^{2}[29]$,

$$
G_{+}=\frac{g_{+}}{Q^{3}}, \quad G_{0}=M \frac{g_{0}}{Q^{4}}, \quad G_{-}=(M)^{2} \frac{g_{-}}{Q^{5}} .
$$

Using further analysis the authors show that the resonance contribution to $g_{1}$ for a given resonance at large $Q^{2}$ can be written as,

$$
g_{1}=\frac{M^{2}}{\pi M_{R} \Gamma_{R}} \frac{g_{+}^{2}}{\left(M_{R}^{2}-M^{2}\right)^{2}}(1-x)^{3} .
$$

Since,

$$
\frac{1}{Q^{2}}=\frac{1}{W^{2}-M^{2}} \frac{1-x}{x} \approx \frac{1}{M_{R}^{2}-M^{2}}(1-x),
$$

with increasing $Q^{2}$, the resonance contributions to structure functions fall-off and also the resonances move to larger $x$ values reaching the limit $x=1$. The authors argue that if this fall-off rate with $x \rightarrow 1$ is the same in the large $Q^{2}$ region and in 
the resonance region, that would indicate duality for structure functions. According to pQCD $g_{1}$ and $F_{1}$ are expected to behave similarly in the deep inelastic region. Since $F_{1} \propto(1-x)^{3}$ in the limit $x \rightarrow 1$, they conclude,

$$
\lim _{x \rightarrow 1} g_{1}(x) \propto(1-x)^{3}
$$

From equations (58) and (60) it is evident that the contribution to $g_{1}$ from the high $Q^{2}$ resonance region is the same as in the deep inelastic region, thereby suggesting duality.

If duality is established, the high $x$ region, which is difficult to measure in DIS, can be studied using measurements made in the resonance region. However duality is expected to fail for the polarized structure function $g_{1}$ in the low $Q^{2}$ region, especially in the $\Delta(1232)$ region, where $A_{1}$ is negative.

\section{$2.4 \quad A_{1}$ IN THE VALENCE REGION}

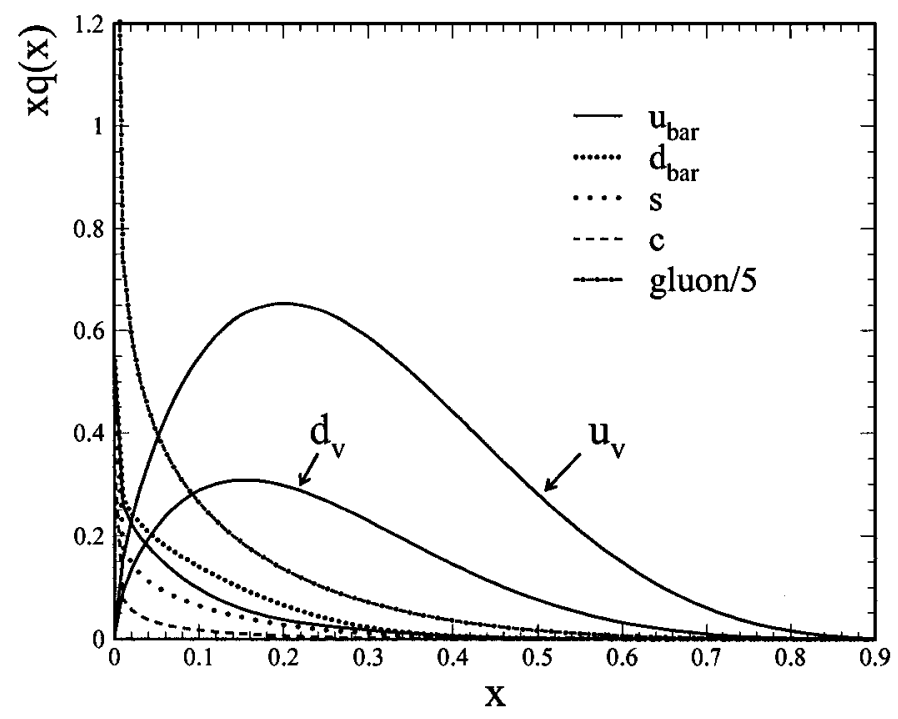

FIG. 12. Parton distributions at $Q^{2}=5 \mathrm{GeV}^{2}$ in CTEQ5M [30] parameterization.

The spin structure functions discussed in section 2.2.2 show a strong $Q^{2}$ dependence in the resonance region and a slow logarithmic $Q^{2}$ dependence in the DIS region. However in the DIS region $A_{1} \approx g_{1} / F_{1}$ shows a minimal $Q^{2}$ dependence since it depends on the ratios of distribution functions. But it should be emphasized that at small $x$ where gluon and sea densities become important $A_{1}$ is expected to be $Q^{2}$ dependent. However, the large $x$ region, where $A_{1}$ is not sensitive to the sea quark 
pairs and gluon densities, is dominated by valence quarks (Fig. 12) and can be used to test different models of nucleon structure.

In this section the physical interpretation of $A_{1}$ in the parton model and other models that can be tested in the valence region will be discussed.

\subsection{1 $A_{1}$ in the Parton Model}

The virtual photon asymmetry $A_{1}$, defined in section 2.1 .3 , has a simple physical interpretation in the parton model. Consider a transverse photon interacting with a quark. The photon can flip the spin of the quark on interaction if the quark spin is antiparallel to the photon spin. However, since quarks are spin $1 / 2$ particles, absorption of a photon by a quark with spin parallel to the photon spin cannot occur (Fig. 13b and 13d). .

(a)

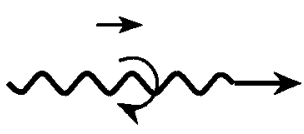

(b)

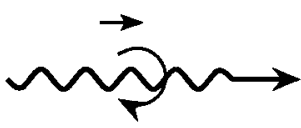

(c)

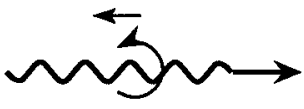

(d)

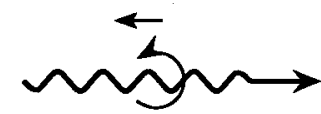

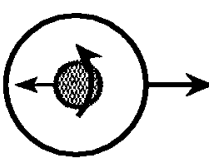
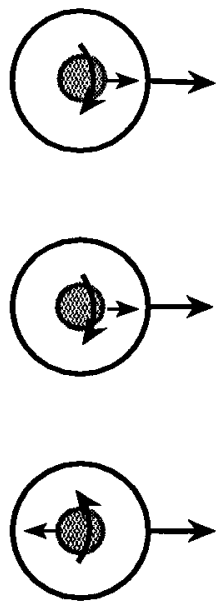

contributes to

$\sigma_{3 / 2}^{\mathrm{T}}$

cannot occur

contributes to

$\sigma_{1 / 2}^{\mathrm{T}}$

cannot occur

FIG. 13. Diagram of polarized deep inelastic scattering. A photon with helicity parallel (antiparallel) to the nucleon spin projection can be absorbed only by quarks with spin projection antiparallel (parallel) to the nucleon spin. The transverse momentum of the quark is assumed to be zero.

Taking the above argument into account, a photon with helicity parallel to the nucleon spin projection on the momentum axis can be absorbed only by quarks with spin projection antiparallel to the nucleon spin (Fig. 13a). Therefore,

$$
\sigma_{\frac{3}{2}}^{T} \sim \sum e_{i}^{2} q_{i}^{\downarrow}(x)
$$


TABLE I. The quantum numbers of the $\mathrm{SU}(3)$ group. Where $I$ is the isospin, $I_{3}$ is the third component of the spin, $S$ the strangeness, $B$ the baryon number and $Q$ is related to $I_{3}, B$ and $S$ by the Gell-Mann-Nishijima relation $Q=I_{3}+\frac{B+S}{2}$.

\begin{tabular}{lccccc}
\hline \hline flavor & charge (Q) & $I$ & $I_{3}$ & $S$ & $B$ \\
\hline $\mathrm{u}$ & $\frac{2}{3}$ & $\frac{1}{2}$ & $\frac{1}{2}$ & 0 & $\frac{1}{3}$ \\
$\mathrm{~d}$ & $-\frac{1}{3}$ & $\frac{1}{2}$ & $-\frac{1}{2}$ & 0 & $\frac{1}{3}$ \\
$\mathrm{~s}$ & $-\frac{1}{3}$ & 0 & 0 & -1 & $\frac{1}{3}$ \\
\hline \hline
\end{tabular}

where $q_{i}^{\downarrow}(x)$ is the quark distribution of the $i^{\text {th }}$ flavor with the helicity antiparallel to the nucleon helicity. Similarly quarks with their helicity parallel to the nucleon helicity can only contribute to $\sigma_{\frac{1}{2}}^{T}$ (Fig. 13c),

$$
\sigma_{\frac{1}{2}}^{T} \sim \sum e_{i}^{2} q_{i}^{\uparrow}(x)
$$

where $q_{i}^{\uparrow}(x)$ is the quark distribution of the $i^{\text {th }}$ flavor with the helicity parallel to the nucleon helicity. Therefor, for $A_{1}$ we get,

$$
A_{1}(x)=\frac{\sum e_{i}^{2}\left[q_{i}^{\uparrow}(x)-q_{i}^{\downarrow}(x)\right]}{\sum e_{i}^{2}\left[q_{i}^{\uparrow}(x)+q_{i}^{\downarrow}(x)\right]}=\frac{\sum e_{i}^{2} \Delta q_{i}(x)}{\sum e_{i}^{2} q_{i}(x)}=\frac{g_{1}(x)}{F_{1}(x)} .
$$

$A_{1}$ can be interpreted as the average (charge-square weighted) polarization of the quarks inside a polarized nucleon.

\subsubsection{Predictions for $A_{1}$}

\section{$\mathrm{SU}(6)$}

The SU(6) symmetric wave function is the simplest model for $A_{1}$. The SU(6) representation is obtained by combining the flavor $\mathrm{SU}(3)$ group (Table I) with the spin $\mathrm{SU}(2)$ group. The fundamental assumption of $\mathrm{SU}(3)$ for baryons is that they are bound states of the three quarks up(u), down(d) and strange(s). When combined with the spin $\mathrm{SU}(2)$ group $\mathrm{SU}(6)$ forms the basis $\mathrm{u} \uparrow, \mathrm{d} \uparrow, \mathrm{s} \uparrow, \mathrm{u} \downarrow, \mathrm{d} \downarrow, \mathrm{s} \downarrow$, where the $\uparrow(\downarrow)$ indicates the spin projection of quarks parallel (antiparallel) to the nucleon spin. 
The overall SU(6) wave function is given by $\Psi=\psi($ space $) \phi($ flavor $) \chi($ spin $) \xi($ color $)$, where $\psi($ space $)$ is the symmetric spatial wave function, $\phi($ flavor $)$ is the $\mathrm{SU}(3)$ flavor wave function, $\chi(\operatorname{spin})$ is the spin $\mathrm{SU}(2)$ wavefunction and $\xi($ color $)$ is the antisymmetric color wave function. The total dimensionality of $\mathrm{SU}(6)$ is given by [31],

$$
6 \bigotimes 6 \bigotimes 6 \bigotimes=56_{S} \bigoplus 70_{M, S} \bigoplus 70_{M, A} \bigoplus 20_{A}
$$

where $A, S$ and $M$ stands for antisymmetric, symmetric and mixed symmetric, which are related to the symmetry properties of the states. In the exact $\mathrm{SU}(6)$ symmetry the proton wave function is given by,

$$
\begin{gathered}
p \uparrow=\frac{1}{\sqrt{2}} u \uparrow(u d)_{S=0}+\frac{1}{\sqrt{18}} u \uparrow(u d)_{S=1}-\frac{1}{3} u \downarrow(u d)_{S=1} \\
-\frac{1}{3} d \uparrow(u u)_{S=1}-\frac{\sqrt{2}}{3} d \downarrow(u u)_{S=1},
\end{gathered}
$$

where $S$ denotes the total spin of the diquark component. The neutron wave function can be obtained by interchanging $u$ and $d$ in the proton wave function. Applying equation (61) to the proton and neutron wavefunctions leads to the predictions,

$$
A_{1}^{p}=\frac{5}{9} ; \quad A_{1}^{n}=0
$$

\section{Hyperfine-perturbed quark model}

In the hyperfine-perturbed quark model SU(6) symmetry is explicitly broken by introducing hyperfine interactions [32], $H_{h y p}$, between each pair of quarks $(i, j)$, which is of the form [33],

$$
H_{h y p}^{i j}=A\left[\frac{8 \pi}{3} \delta^{3}\left(\overrightarrow{r_{i j}}\right) \vec{S}_{i} \cdot \vec{S}_{j}+\frac{1}{r_{i j}^{3}}\left(3 \vec{S}_{i} \cdot \hat{r}_{i j} \vec{S}_{j} \cdot \hat{r}_{i j}-\vec{S}_{i} \cdot \vec{S}_{j}\right)\right]
$$

where $\vec{S}_{i}$ is the spin of the $i^{\text {th }}$ quark, $\overrightarrow{r_{i j}}$ is a vector joining the $i^{\text {th }}$ and $j^{\text {th }}$ quark and $A$ is a constant which depends on the quark masses and the strength of the interaction.

For the s-wave nucleons the ground state $(L=0)$ energies are perturbed only by the Fermi contact term $\vec{S}_{i} \cdot \vec{S}_{j} \delta^{3}\left(\overrightarrow{r_{i j}}\right)$ in $(62)$. In the nucleon rest frame, this perturbation raises the energy of the quark pairs with spin 1 and lowers the energy of pairs with spin 0 . At large $x$, since the struck quark carries most of the energy of the nucleon, the spectator quark pair, which is in a lower energy state, has to be in a spin 0 state. This means that at large $x$ the entire spin of the nucleon should 


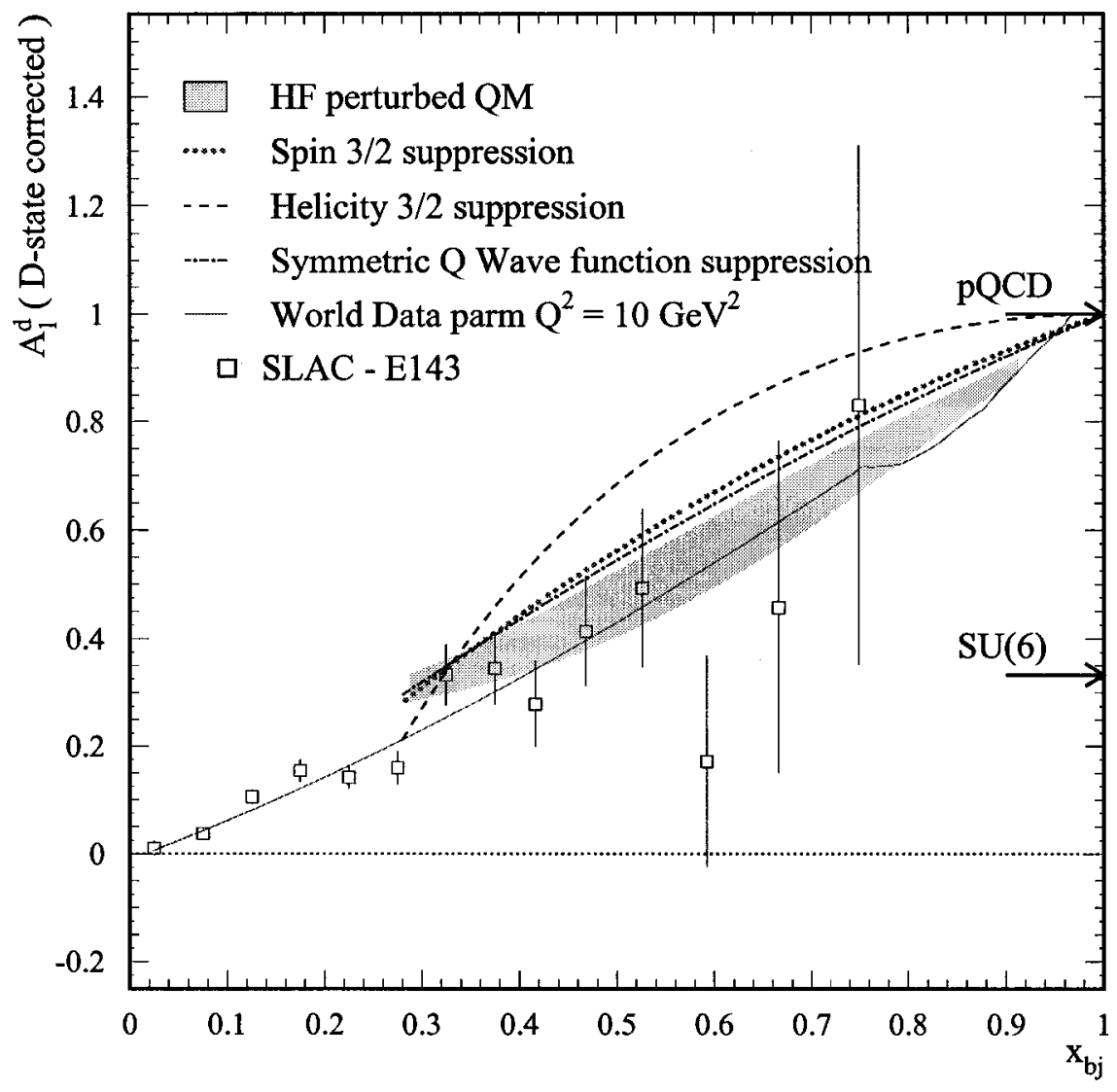

FIG. 14. Predictions for $A_{1}^{d}$ in the valence region. See the text for an explanation of the hyperfine (HP) perturbed quark model(QM) [32] and the three models by Close and Melnitchouk [34]. A parameterization of world data at $Q^{2}=10 \mathrm{GeV}^{2}$ and SLAC E143 [3] data are also included in the plot.

be carried by the struck quark. Therefore, as $x \longrightarrow 1$ the virtual photon asymmetry $A_{1} \longrightarrow 1$. Further analysis shows the following behavior for the distribution functions $[32]$,

$$
\begin{aligned}
& u_{v} \uparrow(x)=\left[1-\frac{1}{2} c_{A}(x)\right] u_{v}(x)-\frac{1}{3}\left[1-c_{A}(x)\right] d_{v}(x), \\
& u_{v} \downarrow(x)=\frac{1}{3}\left[1-c_{A}(x)\right] d_{v}(x)+\frac{1}{2} c_{A}(x) u_{v}(x), \\
& d_{v} \uparrow(x)=\frac{1}{3}\left[1+\frac{1}{2} c_{A}(x)\right] d_{v}(x), \\
& d_{v} \downarrow(x)=\frac{2}{3}\left[1-\frac{1}{4} c_{A}(x)\right] d_{v}(x),
\end{aligned}
$$

with $d(x) / u(x) \simeq \kappa(1-x)$, where $0.5<\kappa<0.6$ and $c_{A}(x)=n x(1-x)^{n}$, with 
$2<n<4$, can be used to predict the behavior of $A_{1}$ in the valence region. The shaded band given in Fig. 14 covers all possible combinations of $\kappa$ and $n$.

\section{Duality}

In a model by F.E. Close and W. Melnitchouk [34] several SU(6) breaking scenarios in the context of quark hadron duality are examined. They look into different cases where certain families of resonances are required to die out at large $Q^{2}$ in order to maintain duality. In particular they consider the suppression at large $x$ of resonances having the following conditions:

1. $\operatorname{spin} 3 / 2(S=3 / 2)$,

2. helicity $3 / 2\left(\sigma_{3 / 2}\right)$ and

3. symmetric wave function,

in the final state of $\gamma+N \longrightarrow N^{*}$, where the initial state is the symmetric $\mathrm{SU}(6)$ wave function and the final states are different states in the $56^{+}$and $70^{-}$dimensional representation in $\mathrm{SU}(6)$. Since the total photoabsorption cross section $\sigma_{1 / 2}+\sigma_{3 / 2}$ is proportional to $F_{1}$ and $\sigma_{1 / 2}-\sigma_{3 / 2}$ is proportional to $g_{1}$, the photoabsorption strengths of transitions from the ground state to each of the final states are incorporated into the model to make predictions for $A_{1} \approx g_{1} / F_{1}$. For each of these cases the final states are summed by giving an appropriate weight to the absorption strengths and the conditions given above are required to be satisfied as $x \longrightarrow 1$. The model predicts ( see Fig. 14) that $A_{1}^{n, p} \longrightarrow 1$ at $x \longrightarrow 1$ for all three conditions.

\section{pQCD}
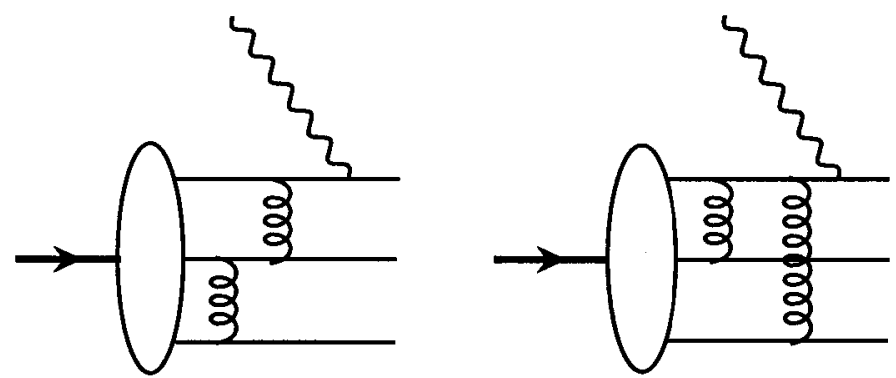

FIG. 15. Two diagrams describing the transfer of momenta from the spectator quark pair to the struck quark as $x \rightarrow 1$. 
Farrar and Jackson [35] have shown that the behavior of structure functions as $x \rightarrow 1$ can be calculated using perturbative QCD methods. As $x \rightarrow 1$ all of the hadron momentum must be carried by one quark. Then they proceeded to show that, in the $Q^{2} \rightarrow \infty$ limit, the minimal number of gluon exchanges required to transfer the momentum of the spectator pair to the struck quark can occur in two ways (Fig. 15). Further they show that these gluon exchanges can only occur if the spectator quark pair have opposite helicities. Therefore, the struck quark must carry the spin of the target nucleon. Hence they obtain the result that $A_{1} \rightarrow 1$ as $x \rightarrow 1$.

\subsection{SUM RULES}

Spin structure functions are often interpreted using sum rules. Most of the spin dependent sum rules are related to the first moment of $g_{1}\left(x, Q^{2}\right)$,

$$
\Gamma_{1}\left(Q^{2}\right)=\int_{0}^{1} g_{1}\left(x, Q^{2}\right) d x
$$

which is sensitive to the spin fraction carried by quarks. In the widely used definition of $\Gamma_{1}$, the elastic contribution is often excluded from the upper limit of the integral. In this section the $Q^{2}$ evolution of $\Gamma_{1}$ and the various sum rules that are related to $\Gamma_{1}$ will be discussed.

\subsubsection{Sum Rules at Kinematic End Points}

Since it was discovered that the nucleon is not an elementary particle, much effort has been devoted to explaining the nucleon spin in terms of its constituents. The main sources that can contribute to the spin of a fast moving nucleon are the intrinsic angular momentum (spins) carried by quarks and gluons and the orbital angular momentum carried by these constituents. Angular momentum conservation requires,

$$
\frac{1}{2} \Delta \Sigma+\Delta G+<L_{z}>=\frac{1}{2}
$$

where $\Delta \Sigma, \Delta G$ and $L_{z}$ are the contributions from the net quark spin, the net gluon spin and the orbital angular momentum, respectively.

At large $Q^{2}, \Delta \Sigma$ can be extracted using the first moment of $g_{1}\left(x, Q^{2}\right)$ along with the known values of nucleon and hyperon beta decay constants. To leading order in the quark parton model, $\Gamma_{1}$ can be written as,

$$
\Gamma_{1}=\int_{0}^{1} g_{1}(x) d x=\frac{1}{2} \int_{0}^{1} \sum e_{i}^{2} \Delta q_{i}(x) d x .
$$


Using isospin symmetry, which requires that,

$$
\begin{gathered}
\Delta u_{p}=\Delta d_{n}=\Delta u, \\
\Delta d_{p}=\Delta u_{n}=\Delta d, \\
\Delta s_{p}=\Delta s_{n}=\Delta s
\end{gathered}
$$

we have,

$$
\begin{aligned}
& \Gamma_{1}^{p}=\int_{0}^{1} g_{1}^{p}(x) d x=\frac{1}{2}\left(\frac{4}{9} \Delta u+\frac{1}{9} \Delta d+\frac{1}{9} \Delta s\right), \\
& \Gamma_{1}^{n}=\int_{0}^{1} g_{1}^{n}(x) d x=\frac{1}{2}\left(\frac{1}{9} \Delta u+\frac{4}{9} \Delta d+\frac{1}{9} \Delta s\right),
\end{aligned}
$$

where $\Delta q_{i}=\int_{0}^{1} \Delta q_{i}(x) d x$ is the fraction of the proton spin carried by the quark flavor $q_{i}$. The net quark spin, $\Delta \Sigma$, is given by,

$$
\Delta \Sigma=\int_{0}^{1} d x \sum \Delta q(x)=\Delta u+\Delta d+\Delta s .
$$

$\Delta \Sigma$ cannot be extracted from DIS data alone. One must use the SU(6) flavor symmetry as well as the weak decay constants $\mathrm{F}$ and $\mathrm{D}$, which are constrained by the hyperon and neutron beta decay measurements. The nucleon axial charges $a_{0}, a_{3}$ and $a_{8}$ are related to $F, D$ and $\Delta \Sigma$ by the relations,

$$
\begin{aligned}
& a_{0}=\Delta u+\Delta d+\Delta s=\Delta \Sigma \\
& a_{3}=\Delta u-\Delta d=F+D \\
& a_{8}=\Delta u+\Delta d-2 \Delta s=3 F-D .
\end{aligned}
$$

By combining equations (75), (76), (79) and (80) we obtain,

$$
\begin{aligned}
& \Gamma_{1}^{p}=\frac{1}{18}[9 F-D+6 \Delta s], \\
& \Gamma_{1}^{n}=\frac{1}{18}[6 F-4 D+6 \Delta s] .
\end{aligned}
$$

By solving equations (79), (80) and (81) for $\Delta u, \Delta d$ and $\Delta s$ the total contribution to the nucleon's spin from the quark spin can be obtained [3]:

$$
\Delta \Sigma=0.31 \pm 0.10
$$

Therefore, the rest of the spin of the nucleon resides either in gluons or in the orbital angular momentum of the nucleon constituents. 
The Bjorken sum rule [2], which was derived using quark current algebra and isospin symmetry, is a fundamental sum rule and has been verified at the level of $10 \%$ accuracy. In its original form it relates the proton neutron difference of $\Gamma_{1}$ to the axial coupling constant $g_{A}$ in neutron beta decay by the relation,

$$
\Gamma_{1}^{p}-\Gamma_{1}^{n}=\frac{g_{A}}{6} .
$$

To $3^{r} d$ order in $\alpha_{s}$ the Bjorken sum rule is given by,

$$
\Gamma_{1}^{p}-\Gamma_{1}^{n}=\frac{g_{A}}{6}\left[1-\left(\frac{\alpha_{s}}{\pi}\right)-3.58\left(\frac{\alpha_{s}}{\pi}\right)^{2}-20.2\left(\frac{\alpha_{s}}{\pi}\right)^{3}\right]+\mathcal{O}\left(\alpha_{s}^{4}\right)
$$

The sum rules discussed so far concern the large $Q^{2}$ behavior of $\Gamma_{1}$. On the other hand, at $Q^{2}=0$ the slope of $\Gamma_{1}$ is constrained by the Gerasimov-Drell-Hearn (GDH) sum rule [4]. The GDH sum rule, which is derived using the dispersion relations for forward Compton scattering and the low-energy theorem, relates the helicity dependent photoabsorption cross sections $\sigma_{1 / 2}$ and $\sigma_{3 / 2}$ to the target anomalous magnetic moment of the nucleon $\kappa$ by the relation,

$$
I_{G D H}=\frac{M^{2}}{8 \alpha \pi^{2}} \int_{\nu_{t h}}^{\infty}\left(\sigma_{\frac{1}{2}}(\nu)-\sigma_{\frac{3}{2}}(\nu)\right) \frac{d \nu}{\nu}=-\frac{1}{4} \kappa^{2},
$$

where $\nu_{t h}$ is the inelastic threshold. $I_{G D H}$ is related to $\Gamma_{1}$ by,

$$
\lim _{Q^{2} \rightarrow 0} \frac{\Gamma_{1}}{Q^{2}}=\frac{I_{G D H}}{2 M^{2}}=-\frac{\kappa^{2}}{8 M^{2}}
$$

which means that $\Gamma_{1}$ must be negative and have a negative slope before turning over to become positive in the deep inelastic scattering region. This has sparked a large interest in measuring $\Gamma_{1}$ in the transition region and is the main focus of the EG1 experiment at CLAS at Jefferson lab.

\subsubsection{Generalized Sum Rules for $\Gamma_{1}$}

In the previous section sum rules related to $\Gamma_{1}\left(Q^{2}\right)$ at the two kinematic end points were discussed. A theoretical tool to extend those sum rules to finite $Q^{2}$ has been suggested by Ji and Osborne [7].

Consider the time ordered forward virtual-photon Compton tensor (Fig. 16),

$$
T^{\mu \nu}=i \int d^{4} \xi e^{i q . \xi}\left\langle P S\left|T J^{\mu}(\xi) J^{\nu}(0)\right| P S\right\rangle .
$$




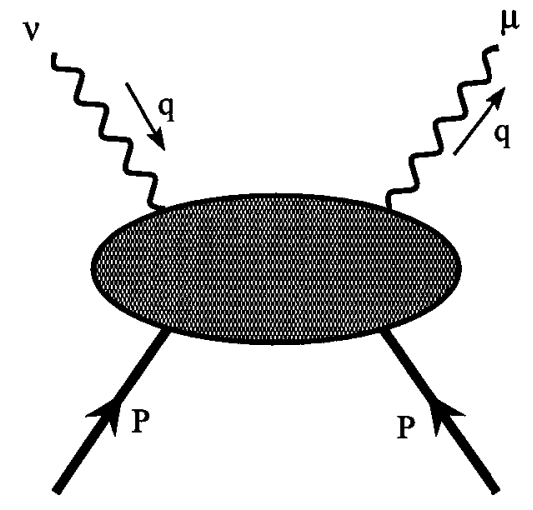

FIG. 16. Forward virtual Compton scattering amplitude.

Using Lorentz symmetry, parity and time-reversal invariance, the spin dependent part of $T^{\mu \nu}$ can be expressed as,

$$
T^{\mu \nu}(P, q, S)=-i \epsilon^{\mu \nu \alpha \beta} q_{\alpha}\left[S_{\beta} S_{1}\left(\nu, Q^{2}\right)+\left(M \nu S_{\beta}-S . q P_{\beta}\right) S_{2}\left(\nu, Q^{2}\right)\right]
$$

where $S_{1,2}\left(\nu, Q^{2}\right)$ are the spin-dependent Compton amplitudes. Since the GDH sum rule can be derived from dispersion relations for the Compton amplitude $S_{1}$ at $Q^{2}=0$, the authors use the same dispersion relations at nonzero $Q^{2}$ to obtain,

$$
S_{1}\left(0, Q^{2}\right)=\frac{8}{Q^{2}} \int_{0}^{1} g_{1}\left(x, Q^{2}\right) d x
$$

The upper limit of the integral includes the elastic contribution. The important feature of this equation is that the right side of the equation (88) is hard to calculate but it can be measured experimentally while the left side of the equation is difficult to measure but it can be calculated theoretically. Therefore theoretical calculations for $S_{1}$ can be experimentally tested by measuring $g_{1}\left(x, Q^{2}\right)$.

\section{$S_{1}\left(0, Q^{2}\right)$ in the parton description}

In the parton description $S_{1}$ can be formalized in the OPE in which the moments are expanded in powers of $1 / Q^{2}$,

$$
\Gamma\left(Q^{2}\right)=\frac{1}{8} Q^{2} S_{1}\left(0, Q^{2}\right)=\sum_{\tau=2,4} \frac{\mu_{\tau}\left(Q^{2}\right)}{\left(Q^{2}\right)^{(\tau-2) / 2}},
$$

where $\tau$ is the twist. The coefficients $\mu_{\tau}\left(Q^{2}\right)$ can be expanded as a perturbation series in the strong coupling constant $\alpha_{s}$. Since the application of OPE requires summing 


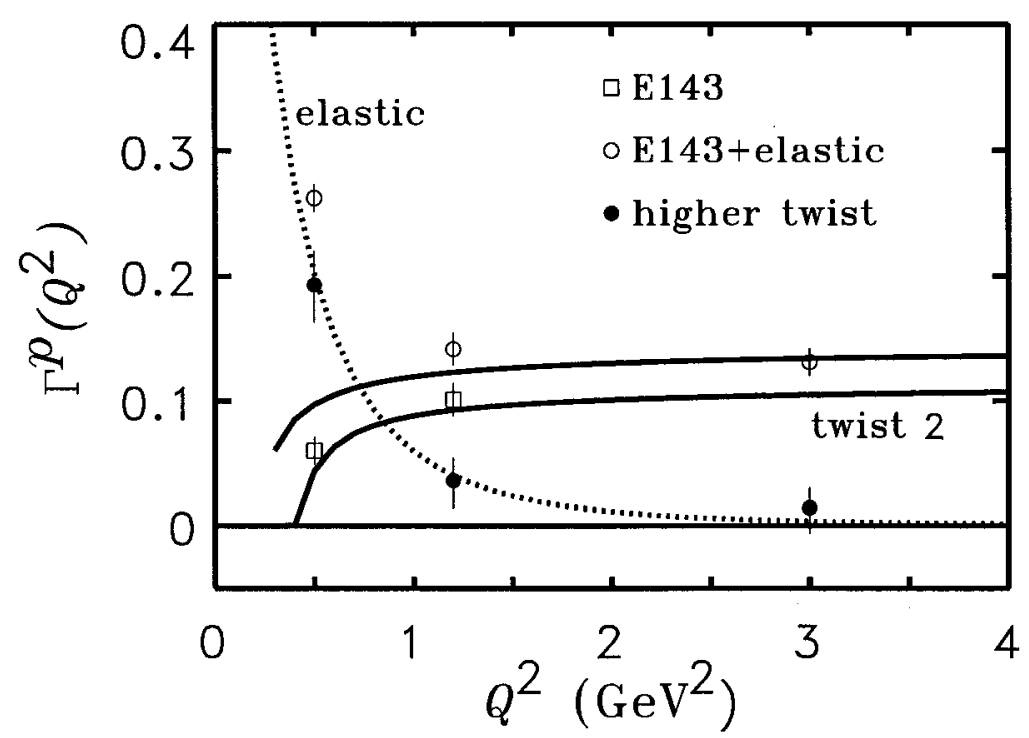

FIG. 17. The plot shows higher twist calculations done by Ji and Melnitchouk [36] for $\Gamma_{1}^{p}$. The squares are the inelastic contribution measured in the E143 experiment and the dashed line shows the elastic contribution to $\Gamma_{1}$ calculated using nucleon form factors. The two solid lines show the upper and lower limit of the twist-2 part calculated using pQCD up to $\mathcal{O}\left(\alpha^{3}\right)$. The solid circles show the higher-twist contribution.

over all final hadronic states, the definition of the first moment in OPE requires the inclusion of elastic components. To obtain $\Gamma_{1}\left(Q^{2}\right)$, the elastic contribution has to be subtracted,

$$
\Gamma_{1}\left(Q^{2}\right)=\Gamma\left(Q^{2}\right)-\Gamma^{e l}\left(Q^{2}\right)
$$

The elastic contribution $\Gamma^{e l}\left(Q^{2}\right)$ can be easily calculated using the known values of electric and magnetic form factors $G_{E}$ and $G_{M}$,

$$
\Gamma^{e l}\left(Q^{2}\right)=\frac{G_{E}\left(Q^{2}\right)\left(G_{E}\left(Q^{2}\right)+\rho G_{M}\left(Q^{2}\right)\right)}{2(1+\rho)},
$$

where $\rho=Q^{2} / 4 M^{2}$. A study of the physical scale parameter that controls the twist expansion, the average parton transverse momentum in the nucleon, shows that the OPE gives a good approximation to $\Gamma_{1}\left(Q^{2}\right)$ down to $Q^{2} \sim 0.5 \mathrm{GeV}^{2}$.

The leading twist term $\mu_{2}\left(Q^{2}\right)$ has been calculated in pQCD using the measured values for $a_{0}, a_{3}$ and $a_{8}$. However higher twist terms are not yet precisely determined due to lack of data in the small $Q^{2}$ region. Evaluation of the $\mu_{4}$ term using existing world data (Fig. 17) has been performed in by Ji and Melnitchouk [36] but the 
authors stressed the need for data in the low $Q^{2}$ region in order to obtain accurate results.

\section{$S_{1}\left(0, Q^{2}\right)$ in the hadron description}

In the hadron description chiral perturbation theory $(\chi \mathrm{PT})$, the effective low-energy theory of the strong interaction, can be used to understand $\Gamma_{1}\left(Q^{2}\right)$ in the $Q^{2}$ region 0 to $0.1 \mathrm{GeV}^{2} . \chi \mathrm{PT}$ is based on chiral symmetry properties of QCD and the effective field theory. In an ideal case where quarks are massless, the QCD Lagrangian is considered to be chiral symmetric, meaning right or left handed quarks will retain their handedness. However this exact chiral invariance is spontaneously broken by small quark mass terms allowing left and right handed quarks to mix, inducing very light pseudo-Goldstone bosons, which are much lighter than their hadronic counterparts. This allows one to make corrections to the chiral symmetry predictions of hadronic observables at small energy-momenta in a perturbative manner via an effective field theory- $\chi \mathrm{PT}$.

Since the common definition of $\Gamma_{1}\left(Q^{2}\right)$ does not include the elastic contribution, the authors [7] use a subtracted version of $S_{1}$ in the low $Q^{2}$ region,

$$
\bar{S}_{1}\left(0, Q^{2}\right)=S_{1}\left(0, Q^{2}\right)-S_{1}^{e l}\left(0, Q^{2}\right),
$$

where $S_{1}^{\text {el }}\left(0, Q^{2}\right)$ is the elastic contribution. $\bar{S}_{1}\left(0, Q^{2}\right)$ can be expanded around $Q^{2}=$ 0

$$
\frac{8}{Q^{2}} \Gamma_{1}\left(Q^{2}\right)=\bar{S}_{1}\left(0, Q^{2}\right)=-\frac{\kappa^{2}}{M^{2}}+\bar{S}_{1}^{\prime}(0,0) Q^{2}+\frac{1}{2} \bar{S}_{1}^{\prime \prime}(0,0) Q^{4}+\ldots \ldots \ldots,
$$

where $\bar{S}_{1}^{i}$ can be expanded in chiral perturbation theory in a power series of pion mass $m_{\pi}$,

$$
\bar{S}_{1}^{\prime}(0,0)=\frac{a}{m_{\pi}^{2}}+\frac{b}{m_{\pi}}\left(\log \left(m_{\pi}\right)+\beta\right)+\ldots . .
$$

The leading chiral contribution (Fig. 18) calculated in heavy baryon chiral perturbation theory (HB $\chi \mathrm{PT})$ [7] yields $a=0$. Therefore, to leading order $\bar{S}_{1}\left(0, Q^{2}\right)$ is $Q^{2}$ independent. However calculations at next-to-leading order in momenta, $\mathcal{O}\left(p^{4}\right)$, show a strong $Q^{2}$ dependence [37],

$$
\begin{aligned}
& 2 M^{2} \Gamma_{1}^{p}\left(Q^{2}\right)=\frac{\kappa_{p}^{2}}{4} Q^{2}+6.85 Q^{4}+\ldots . . \\
& 2 M^{2} \Gamma_{1}^{n}\left(Q^{2}\right)=\frac{\kappa_{n}^{2}}{4} Q^{2}+5.54 Q^{4}+\ldots . .
\end{aligned}
$$



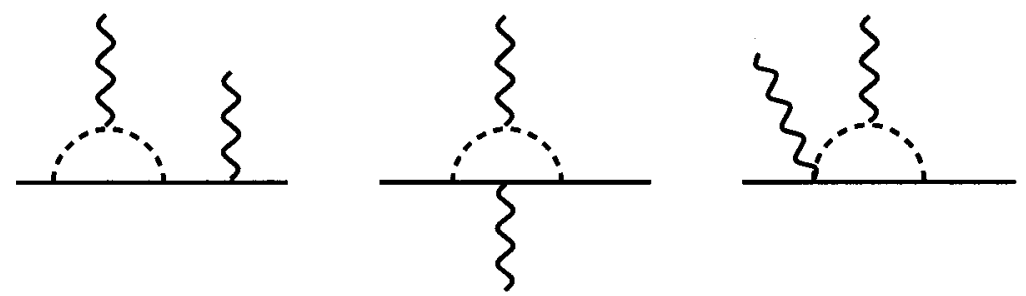

FIG. 18. One-loop diagrams which contribute to $\chi \mathrm{PT}$ calculations (leading order).

\section{Transition region}

There is a small region of $Q^{2}$ from 0.1 to $\sim 0.5 \mathrm{GeV}^{2}$ in which we still do not have a firm theoretical understanding. This is the region where the transition between parton and hadron degrees of freedom happens. However Ji and Osborne [7] point out that the forward virtual-photon Compton tensor can be transformed into Euclidian space making it possible to calculate $S_{1}$ in lattice QCD. Nevertheless, lattice QCD, in which the space time continuum is replaced with a hypercubic lattice by restricting quarks and gauge fields to the lattice nodes, uses numerical methods to do calculations and requires large computing power making it somewhat time consuming. Therefore, we still do not have any theoretical predictions in this $Q^{2}$ region. There is also the possibility of using some extension of the higher twist expansion and $\chi \mathrm{PT}$ with appropriate higher order terms to close the gap between the two regions [7].

\subsubsection{Phenomenological Models}

As shown in the previous section, there are some kinematic regions where we still do not have a good theoretical understanding. Because of that there are some attempts to model $\Gamma_{1}$ in the resonance region. In this section two models with which we compare our results will be discussed.

\section{Model by Burkert and Ioffe}

The model by Burkert and Ioffe [6] is a combination of resonance contributions and a $1 / Q^{2}$ parameterization;

$$
\frac{2 M^{2}}{Q^{2}} \Gamma_{1}\left(Q^{2}\right)=I_{G D H}\left(Q^{2}\right)=I^{r e s}\left(Q^{2}\right)+I^{\prime}\left(Q^{2}\right),
$$


where $I^{r e s}\left(Q^{2}\right)$ is the resonance contribution which is modeled up to $W=1.8 \mathrm{GeV}$ using meson electroproduction data. The integral $I_{G D H}\left(Q^{2}\right)$ at small $Q^{2}$ is mostly saturated by contributions from low-lying resonant states and the resonance contribution decreases rapidly with increasing $Q^{2}$. The second term, $I^{\prime}\left(Q^{2}\right)$, has the following parameterization,

$$
I^{\prime}\left(Q^{2}\right)=2 M^{2} \Gamma^{a s}\left[\frac{1}{Q^{2}+\mu^{2}}-\frac{c \mu^{2}}{\left(Q^{2}+\mu^{2}\right)^{2}}\right],
$$

where $\Gamma^{a s}=\Gamma_{1}\left(Q^{2} \rightarrow \infty\right)$ has been used as a constraining parameter at large $Q^{2}$ and $\mu$, which characterizes the scale of the $Q^{2}$ variation, is the $\rho$ (or $\omega$ ) mass. The variable $\mathrm{c}$ is then determined by using the GDH sum rule at $Q^{2}=0$,

$$
I(0)=I_{1}^{r e s}(0)+I_{1}^{\prime}(0)=-\frac{1}{4} \kappa^{2},
$$

which gives,

$$
c=1+\frac{1}{2} \frac{\mu^{2}}{M_{N}^{2}} \frac{1}{\Gamma^{a s}}\left[\frac{1}{4} \kappa^{2}+I^{r e s}(0)\right] .
$$

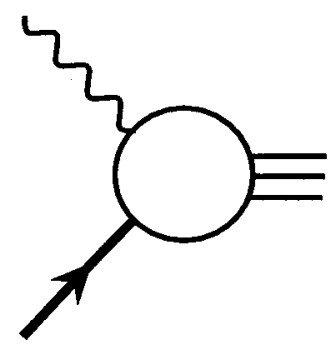

(a)

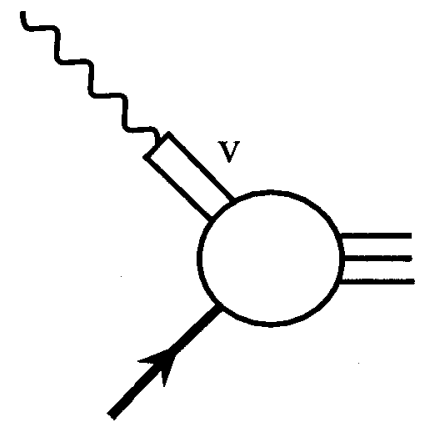

(b)

FIG. 19. Diagrams representing formulations of the VDM. (a) Direct coupling of the photon to the hadron. (b) Coupling of the photon to the hadron through a vector meson $\mathrm{v}$.

The authors argue that the two terms in the parameterization for $I^{\prime}\left(Q^{2}\right)$ can be explained using the Vector Dominance Model (VDM). VDM assumes that the photon couples to the hadron by fluctuating into a vector meson with the same quantum numbers as the photon. The authors point out that the second term in equation (97) can be explained using diagrams in which the photon couples to the hadron through vector mesons (Fig. 19b) and the first term corresponds to cases where one of the photons couples directly to the hadron and the second photon couples to the hadron 
through a vector meson (Fig. 19a and 19b). However it is important to point out that the constants in equation (97) have not been calculated using the VDM. The predictions for $\Gamma_{1}$ by Burkert and Ioffe is shown for the deuteron in Fig. 20.

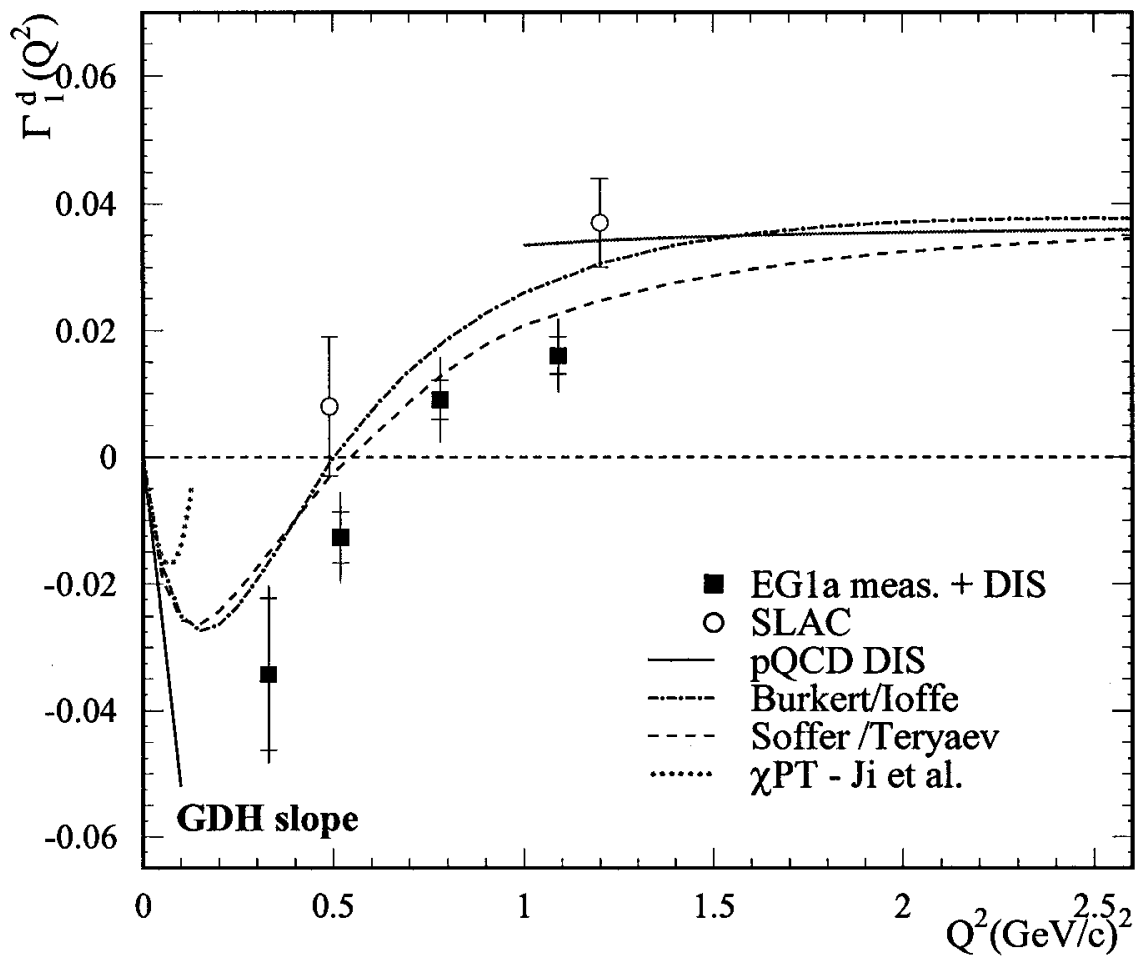

FIG. 20. Predictions for $\Gamma_{1}^{d}$. Details of each curve are given in the text. Previous CLAS data [40] and SLAC E143 [3] data are also plotted.

\section{Model by Soffer and Teryaev}

The model by Soffer and Teryaev [38] is an interpolation of the integral $\Gamma_{1+2}\left(Q^{2}\right)=$ $\Gamma_{1}\left(Q^{2}\right)+\Gamma_{2}\left(Q^{2}\right)$, where

$$
\Gamma_{2}\left(Q^{2}\right)=\int_{0}^{1} g_{2}\left(x, Q^{2}\right) d x .
$$

As $Q^{2} \longrightarrow \infty, \Gamma_{2}$ is given by the well known Burkhardt-Cottingham sum rule,

$$
\int_{0}^{1} g_{2}(x) d x=0
$$

Therefore at large $Q^{2}$ the sole contribution to $\Gamma_{1+2}\left(Q^{2}\right)$ comes from the asymptotic value of $\Gamma_{1}$ which is known from previous experiments. The $Q^{2}$ dependence of $\Gamma_{2}$ is given by the Schwinger sum rule,

$$
\frac{2 M^{2}}{Q^{2}} \Gamma_{2}\left(Q^{2}\right)=I_{2}\left(Q^{2}\right)=\frac{1}{4} \mu G_{M}\left(Q^{2}\right) \frac{\mu G_{M}\left(Q^{2}\right)-G_{E}\left(Q^{2}\right)}{1+Q^{2} / 4 M^{2}},
$$


where $\mu$ is the nucleon magnetic moment and $G_{E}$ and $G_{M}$ are nucleon electric and magnetic form factors. At $Q^{2}=0 I_{2}$ can be obtained from $I_{2}(0)=\frac{\kappa^{2}+e \kappa}{4}$ [38], and $I_{1}(0)$ is given by the GDH sum rule. Therefore we obtain $I_{1+2}(0)=\frac{e \kappa}{4}$ which is positive. Since $I_{1+2}$ is known at $Q^{2}=0$ and at large $Q^{2}$ the authors use a simple parameterization in the intermediate $Q^{2}$ region. Then $\Gamma_{1}\left(Q^{2}\right)$ can be easily deduced from $\Gamma_{1}\left(Q^{2}\right)=\Gamma_{1+2}\left(Q^{2}\right)-\Gamma_{2}\left(Q^{2}\right)$. The main advantage of using $\Gamma_{1+2}$ instead of $\Gamma_{1}$ is that $\Gamma_{1+2}$ is positive at $Q^{2}=0$ and at large $Q^{2}$ which makes the parameterization much smoother since it avoids a sign change in the intermediate region as required by $\Gamma_{1}$ (Fig. 20). Unfortunately, the extension of this model to the neutron is not straightforward since $I_{1+2}(0)=0$ in this case.

\subsection{DEUTERON AS A PROTON+NEUTRON TARGET}

\subsubsection{State Correction}

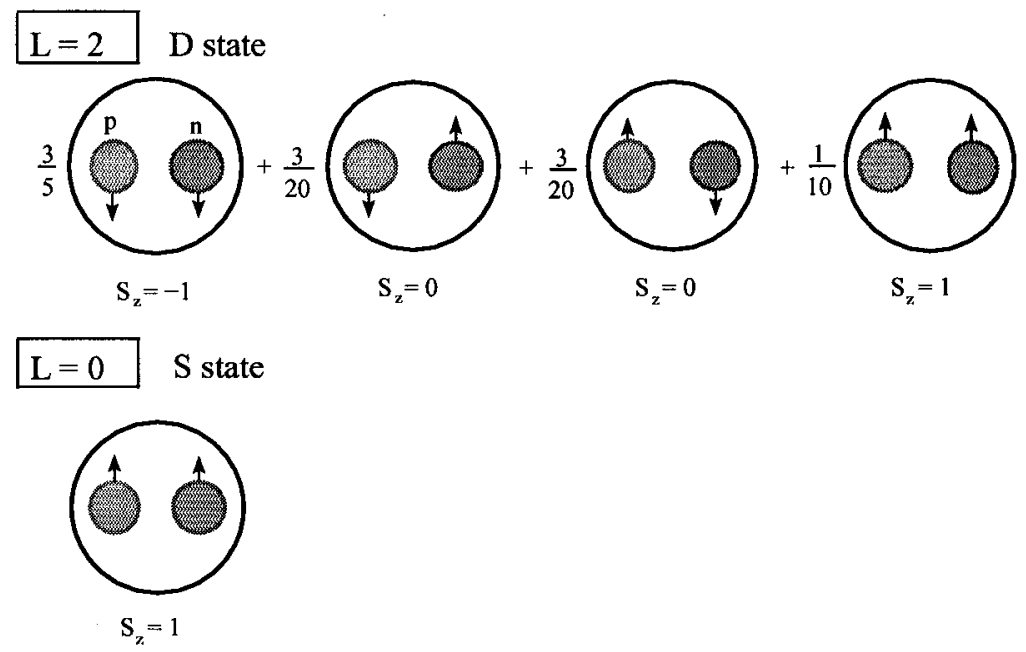

FIG. 21. Spin of the deuteron as a combination of the proton and the neutron spin. The two possible angular momentum states $L=0$ and $L=2$ are shown. The $\mathrm{z}$ projection of the angular momentum for each $L=2$ configuration found in Eq. (99).

The formalism explained so far describes photon absorption on a free nucleon. However this work is based on scattering from a deuteron target which is a bound state of a proton and a neutron. The deuteron is a particle with total angular momentum $J=1,+$ parity and the spin $S=1$. Therefore the deuteron can have orbital angular momentum is zero (S state) or two (D state). In the $\mathrm{S}$ state,

$$
\left|J=1 ; J_{z}=1\right\rangle=\left|L=0, L_{z}=0\right\rangle\left|S=1, S_{z}=1\right\rangle .
$$


Therefore, in this state the spin of the proton and the neutron are aligned with the deuteron spin. However in the D state z projection of the spin is not always aligned with the total angular momentum,

$$
\begin{aligned}
\left|J=1 ; J_{z}=1\right\rangle & =\sqrt{\frac{3}{5}}\left|L=2 ; L_{z}=2\right\rangle\left|S=1 ; S_{z}=-1\right\rangle \\
& -\sqrt{\frac{3}{10}}\left|L=2 ; L_{z}=1\right\rangle\left|S=1 ; S_{z}=0\right\rangle \\
& +\sqrt{\frac{1}{10}}\left|L=2 ; L_{z}=0\right\rangle\left|S=1 ; S_{z}=1\right\rangle .
\end{aligned}
$$

Therefore, for the D state, the following three situations have to be considered:

- The case where both nucleon spins are aligned with the deuteron spin which has a probability of $1 / 10$.

- The case where both nucleon spins have spin opposite to the deuteron spin, which has a probability of $3 / 5$.

- The case where one of the nucleon spins is aligned and the other one is antialigned with the deuteron spin which has a probability of $3 / 10$.

The probability of finding the deuteron in the $\mathrm{D}$ state, $\omega_{d}$, is approximately 0.056 . Therefore the probability of finding a nucleon with spin down is $\omega_{d}\left(\frac{1}{2} \frac{3}{10}+\frac{3}{5}\right)=\frac{3}{4} \omega_{d}$. If Fermi smearing and binding effects are ignored, the following relations between the deuteron and nucleon cross sections can be obtained,

$$
\begin{aligned}
& \sigma_{d}^{\uparrow \downarrow}=\left(1-\frac{3}{4} \omega_{d}\right) \sigma_{N}^{\uparrow \downarrow}+\frac{3}{4} \omega_{d} \sigma_{N}^{\uparrow \uparrow}, \\
& \sigma_{d}^{\uparrow \uparrow}=\left(1-\frac{3}{4} \omega_{d}\right) \sigma_{N}^{\uparrow \uparrow}+\frac{3}{4} \omega_{d} \sigma_{N}^{\uparrow \downarrow},
\end{aligned}
$$

where the first arrow indicates the lepton beam helicity and the second arrow the spin direction of the target. The nucleon cross section $\sigma_{N}$ is given by,

$$
\sigma_{N}=\frac{1}{2}\left(\sigma_{p}+\sigma_{n}\right)
$$

When substituted into equation (26) we obtain,

$$
A_{1}^{d}=\left(1-\frac{3}{2} \omega_{d}\right)\left(\frac{\sigma_{p}}{\sigma_{d}} A_{1}^{p}+\frac{\sigma_{n}}{\sigma_{d}} A_{1}^{n}\right) .
$$

By replacing the cross sections with the corresponding $F_{2}$ we obtain,

$$
A_{1}^{d}(x)=\frac{1-\frac{3}{2} \omega_{d}}{1+F_{2}^{n}(x) / F_{2}^{p}(x)}\left(A_{1}^{p}(x)+\frac{F_{2}^{n}(x)}{F_{2}^{p}(x)} A_{1}^{n}(x)\right) .
$$

Similarly $g_{1}^{d}$ can be written as,

$$
g_{1}^{d}\left(x, Q^{2}\right)=\left(1-\frac{3}{2} \omega_{d}\right)\left[g_{1}^{p}\left(x, Q^{2}\right)+g_{1}^{n}\left(x, Q^{2}\right)\right] .
$$




\subsubsection{Nuclear-binding Effects}

In addition to the $\mathrm{D}$ state correction explained above the extraction of neutron results from the deuteron requires correction for nuclear binding effects in the deuteron. An overview of some of those corrections is given in the following section.

- EMC effect : This effect takes into account the distortion of the structure of a free-nucleon by a nuclear medium. This correction is usually observed as the deviation of the ratio $r_{E M C}=\sigma_{A} / \sigma_{D}$ from unity. Here $\sigma_{A}$ is the pernucleon cross section of a nucleus, which has an atomic number (A) greater than two, and $\sigma_{D}$ is the per nucleon cross section for a deuteron. This effect was first observed in lepton scattering cross sections for iron and deuterium by the European Muon Collaboration (EMC) [39].

- Fermi motion : This correction arises due to the fact that the bound nucleons are moving inside the nucleus and thus have a momentum distribution relative to the center-of-mass energy of the nucleus. This causes the center-of-mass energy seen by the constituent nucleons to shift by a "Doppler effect." In the kinematic regions where the free nucleon cross sections are strongly energy dependent (low $Q^{2}$, large $x$ ), this effect becomes large causing a smearing effect in cross sections.

- Binding (off-shelf) effects : The proton and neutron inside the deuteron are bound together with a binding energy of $2.2 \mathrm{MeV}$. Therefore the mass of the deuteron $\left(M_{d}\right)$ can be written in terms of the masses of the proton $\left(M_{p}\right)$ and neutron $\left(M_{n}\right)$ as,

$$
M_{d}=M_{p}+M_{n}-2.2 \mathrm{MeV},
$$

which means that both nucleons cannot be on the mass shell at the same time. Therefore a correction has to be made to take into account the photon interaction with an off shell nucleon.

- Non-nucleonic d.o.f : There are also effects from pions and $\Delta$ in the deuteron wave function plus potentially exotic effects such as six quark bags.

These effects make a direct extraction of $A_{1}^{n}$ or $g_{1}^{n}$ from equations (103) and (105) challenging and somewhat model dependent.

The existing CLAS deuteron data (part of the EG1a experiment) [40] are the highest resolution spin structure data, for the deuteron, in the resonance region to 


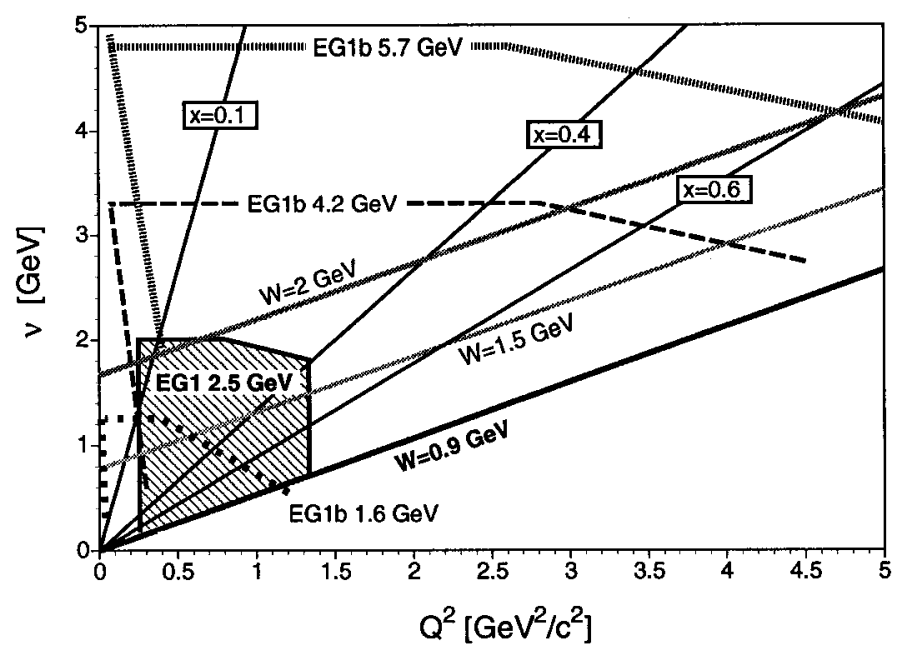

FIG. 22. Kinematic coverage of the EG1a data [40] and the EG1b data. Details are in the text.

date. However, as can be seen from Fig. 20 the precision of the data is not good enough to make accurate conclusions. The second part of the same experiment known as EG1b, which is the main focus of this thesis, has taken ten times more data in the same kinematic region and also has a wider kinematic coverage both towards smaller and larger $Q^{2}$ values (Fig. 22). The focus of this work is on measuring $A_{1}, g_{1}$ and $\Gamma_{1}$ for the deuteron. In this thesis, data covering a $Q^{2}$ range from $0.05 \mathrm{GeV}^{2}$ to 4.2 $\mathrm{GeV}^{2}$, based on data taken at the beam energies $1 . x \mathrm{GeV}$ and $5 . x \mathrm{GeV}$ ( $x$ stands for slightly different beam energies) will be discussed. These measurements complement the data at the photoabsorption point and in the deep inelastic scattering region. This opens up the possibility of studying the transition from hadronic to quark degrees of freedom over a wide range of $Q^{2}$. Details of the experiment are presented in the next chapter. 


\section{CHAPTER 3}

\section{EXPERIMENTAL SETUP}

The EG1 experiment took place at the Thomas Jefferson National Accelerator Facility (TJNAF), where the Continuous Electron Beam Accelerator Facility (CEBAF) delivers beam to three experimental halls. The EG1 experiment took place in experimental Hall B, which houses the CEBAF Large Acceptance Spectrometer (CLAS). The experiment was designed to probe the spin structure of the nucleons, the proton and the neutron, at low to moderate $Q^{2}$ values. Therefore in addition to the CLAS, a polarized target was also required. In this chapter, a brief overview of all the equipments used in the experiment will be discussed.

\subsection{THE ACCELERATOR AND THE POLARIZED BEAM}

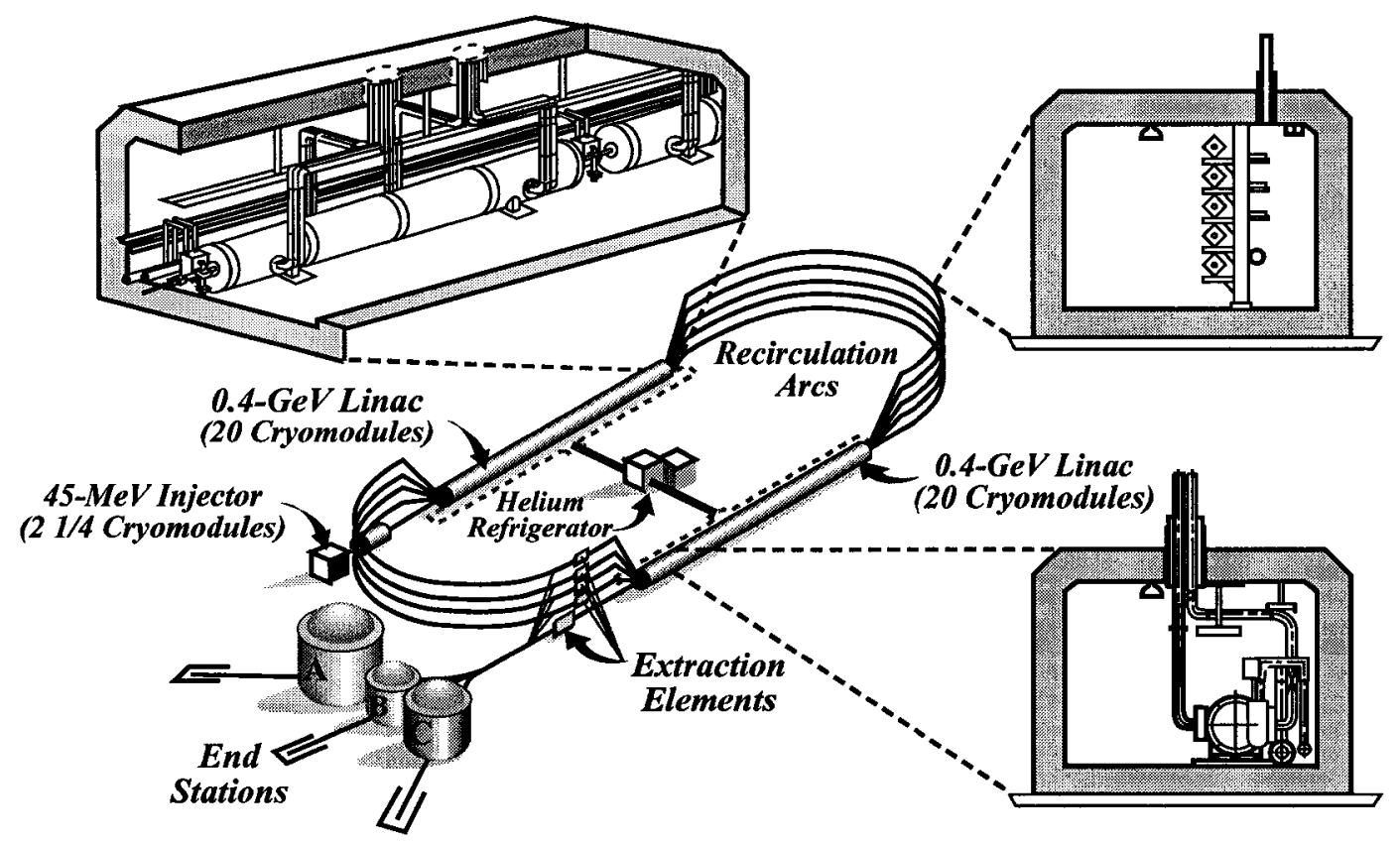

FIG. 23. The Thomas Jefferson National Accelerator Facility.

The CEBAF is composed of two linear accelerators (linacs), five sets of recirculating arcs and a $45 \mathrm{MeV}$ injector. The beam can travel through the two linacs up to five times before delivery to the three experimental halls. The accelerator is capable of providing different beam energies to the three experimental halls simultaneously. It can provide beam energies between $800 \mathrm{MeV}$ and $5.8 \mathrm{GeV}$ (there are plans for a 
future upgrade to $12 \mathrm{GeV}$ ). It is designed to probe the structure of the nucleon and the nucleus over a large range of $Q^{2}$, encompassing the quark and parton degrees of freedom.

The layout of the accelerator is shown in Fig. 23. The beam begins at the injector. The linacs are made of superconducting radio-frequency (SRF) cavities. In total $338 \mathrm{SRF}$ cavities were used to build the linacs. Each of these cavities is grouped into 8 , to make a cryomodule. All cryomodules are held in a $2 \mathrm{~K}$ helium bath, which keeps the accelerator cavities superconducting. Cavities are operated by injecting an oscillating electromagnetic field such that the electron always sees a net positive charge in front of it (Fig. 24) which forces the electrons to accelerate continuously throughout the cavity. The two linacs are connected to two $180^{\circ}$ arc

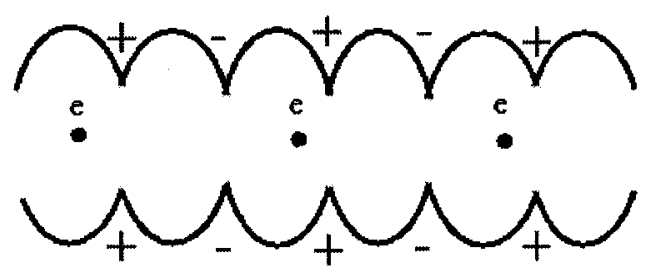

FIG. 24. Charge distribution in the RF cavities at one instant. When the electron moves forward the oscillating electromagnetic field induces a positive charge in front of the electron thereby accelerating it continuously when it is traveling in the cavity.

sections so that the beam can be accelerated up to five times through the two linacs before it is delivered to the three experimental halls. The energy and the phase of the cavities are identical in the two linacs. To bend the beam and keep it focused as it passes through the arcs, several quadrupole and dipole magnets are used. The beam, accelerated to the required beam energy, is chopped and every third pulse is delivered to each experimental hall. These bunches have a relative phase of $120^{\circ}$ and a bunch length of $1.7 \mathrm{ps}$.

Depending upon the experiment, the electrons can be generated either in a polarized state from a DC photo-emission gun using a cathode, or in an unpolarized state from a thermonic gun. The polarized electron source consists of layers of GaAs combinations in which pure GaAs is grown on top of $\mathrm{GaAs}_{0.72} \mathrm{P}_{0.28}$ and some other combinations of GaAs. Using a GaAs combination has been used to successfully increase the polarization over pure GaAs. The emission of electrons from a semiconductor material can be divided into three steps.

1. Circularly polarized photons are absorbed by the material. 
2. The high concentration of electrons that are in the valence band are moved to the surface.

3. The electrons are emitted from the surface.

The circularly polarized light, which is directed on to the cathode, is produced by sending the laser light through a Pockels cell, which is an electro-optical crystal that converts linearly polarized light to circularly polarized light. The helicity of the directed light can be varied by changing the voltage of the Pockels cell, which can be used to change the helicity of the emitted electrons. The energy level diagram and transition probabilities of a strained-lattice GaAs cathode is given in Fig. 25. When the proper thickness of GaAs is grown on top of $\mathrm{GaAs}_{0.72} \mathrm{P}_{0.28}$ the resulting strain induces a gap in the $P_{3 / 2}$ energy levels, whereas all the energy levels are degenerate in a pure GaAs cathode. Therefore, if the cathode is illuminated with circularly polarized laser light with energy between $E_{g}$ and $E_{g}+\delta$, the electrons from the energy level $P_{3 / 2}, m_{j}=+3 / 2$ excite into the conduction band and subsequently escape into the surrounding vacuum. However, because of the energy gap, $\delta$, the electrons from the $P_{3 / 2}, m_{j}=+1 / 2$ energy level are not excited by the laser light. Therefore in principle one can achieve a beam polarization of $100 \%$.

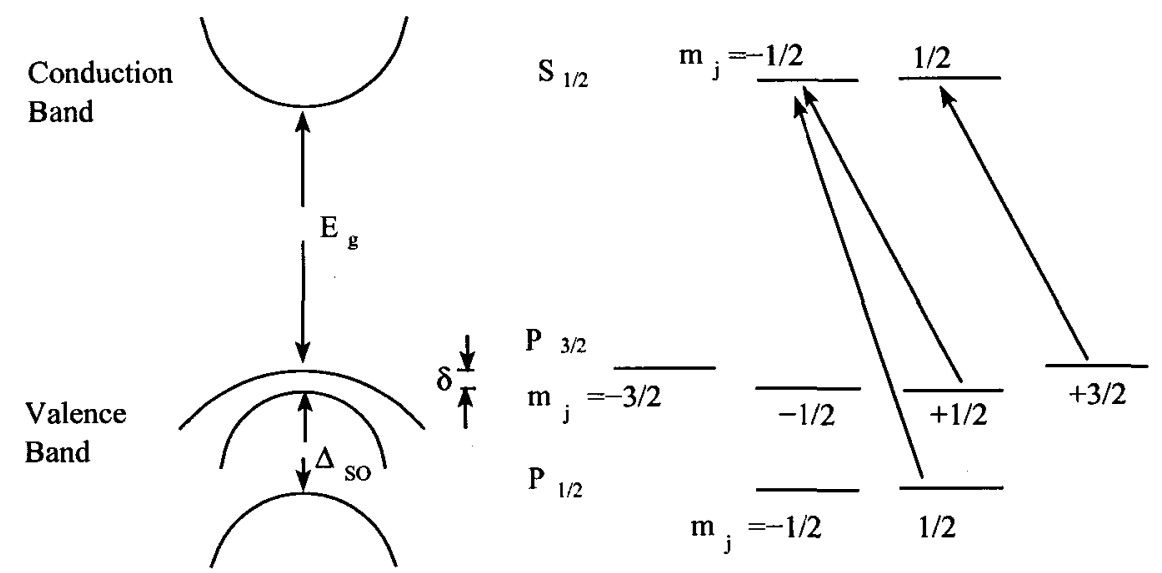

FIG. 25. The energy level diagrams for GaAs grown on a combination of GaAs. Details are in the text.

The spin orientation of the injected electrons are controlled by a Wien Filter, which consists of electric and magnetic fields that are transverse to the direction of motion of the electron. The resulting polarization was measured using a Mott polarimeter. However the beam polarization was also frequently measured once it 
entered the experimental hall using a Moller polarimeter, which will be discussed in detail in section 3.2 .

\subsection{HALL B BEAM LINE DEVICES}

Most of the beam line devices, which includes the harp, beam position monitors, Moller polarimeter and Faraday cup, are used to steer the beam and to monitor the quality of the beam that is delivered to the hall. In this section a very brief overview of some of these devices is discussed.

\section{Electron beam position monitors}

Continuous monitoring of the beam delivered to Hall $B$ is done by three beam position monitoring devices (BPMs) that are located $36.0 \mathrm{~m}, 24.6 \mathrm{~m}$ and $8.2 \mathrm{~m}$ upstream of the Hall B spectrometer, CLAS. These BPMs provide the position and the intensity of the beam at the above mentioned positions and are read at a rate of $1 \mathrm{~Hz}$.

\section{Beam profile and quality monitors: Harps}

The Harp is a system of thin wires which measures the beam position in the $x$ and $y$ direction. This is performed by moving the wires through the beam with the use of a stepper motor and by measuring the Cerenkov light produced by scattered electrons in photomultiplier tubes (Fig. 26). These measurements are very useful in tuning the beam after major changes are made. Since the beam has to be intercepted, a Harp scan can be only done when the data taking is not in progress.

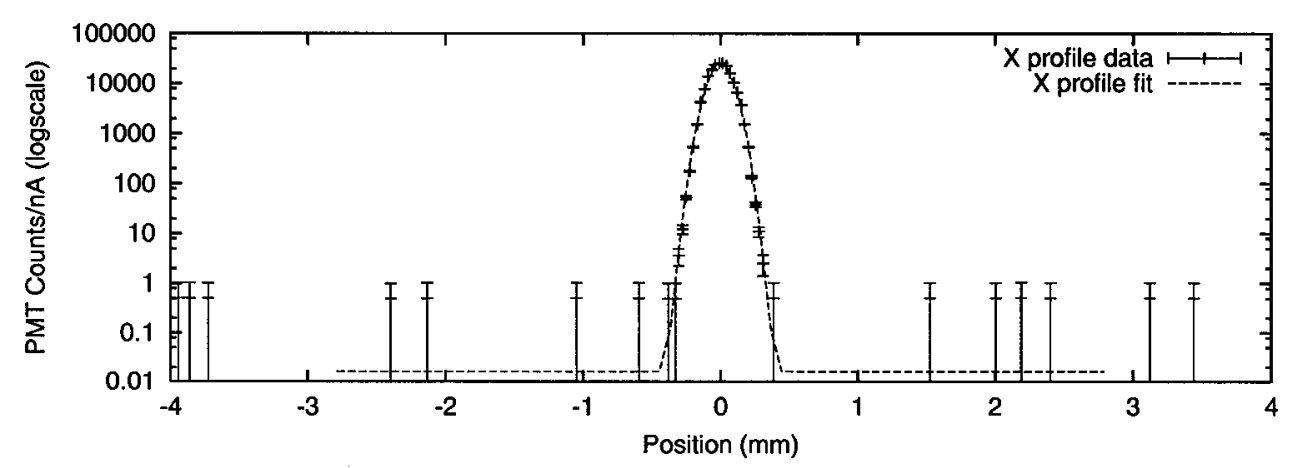

FIG. 26. The X profile of the beam obtained using a Harp scan [41]. 


\section{The Faraday Cup}

The Faraday cup enables precise measurements of the integrated beam charge, which is used in determining the flux of the beam. By gating the Faraday cup signal on the beam helicity it is possible to measure the beam charge asymmetry, which arises when there is more current in one helicity state than another.

\section{Moller polarimeter}

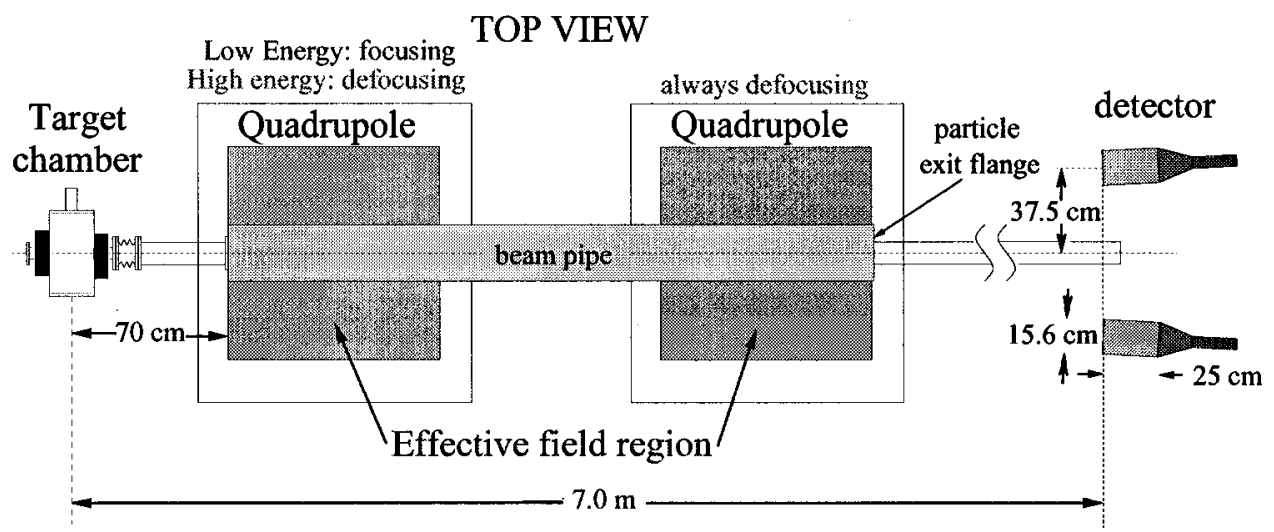

FIG. 27. A view from the top of the Hall B Moller polarimeter [41].

The Moller polarimeter is designed to measure the beam polarization. The statistical uncertainty of a Moller measurement, which runs for about 30 minutes, is about 1\%. The polarization is measured by scattering polarized electrons in the beam off polarized atomic electrons in the target foil. The interaction between the beam and the target depends on the polarization of both the beam, $P^{b}$ and the target, $P^{t}$.

$$
\frac{d \sigma}{d \Omega} \propto\left(1+\sum_{i, j=x, y, z} P_{i}^{t} A_{i j} P_{j}^{b}\right),
$$

where $P_{i}$ are the components of the electron polarization on the $x, y$ and $z$ axis and $A_{i j}$ are asymmetry parameters, which depend on the scattering angle in the center of mass $(\mathrm{CM})$ frame, $\theta_{C M}$. If we consider only the electrons scattered at $\theta_{C M}=90^{\circ}$ and further assume the electron beam is in the $z$ direction and the scattering occurs in the $X Z$ plane, the asymmetry parameters $A_{x x}, A_{y y}$ and $A_{z z}$,

$$
\begin{aligned}
& -A_{x x}=A_{y y}=\frac{\sin ^{4} \theta_{C M}}{\left(3+\cos ^{2} \theta_{C M}\right)^{2}} \\
& -A_{z z}=\frac{\left(7+\cos ^{2} \theta_{C M}\right) \sin ^{2} \theta_{C M}}{\left(3+\cos ^{2} \theta_{C M}\right)^{2}}
\end{aligned}
$$


are maximum and the other asymmetry parameters are negligible. Therefore, with the precise knowledge of the target polarization parameter on the three axes, the polarization of the beam can be extracted.

The Hall B Moller polarimeter which is shown in Fig. 27, consists of a target chamber, two quadrupole magnets and two detectors. The target, which is a foil, is magnetized using a coil system. The two quadrupoles are used for separating the scattered electrons from the unscattered beam. The two detectors are located downstream of the target. The number of coincidences from the two detectors is recorded for each helicity state and is used to calculate the asymmetry, which subsequently is used to determine the beam polarization.

During the EG1 running period the beam polarization was frequently measured using the Moller polarimeter and was between $60 \%$ and $80 \%$.

\subsection{THE CLAS}

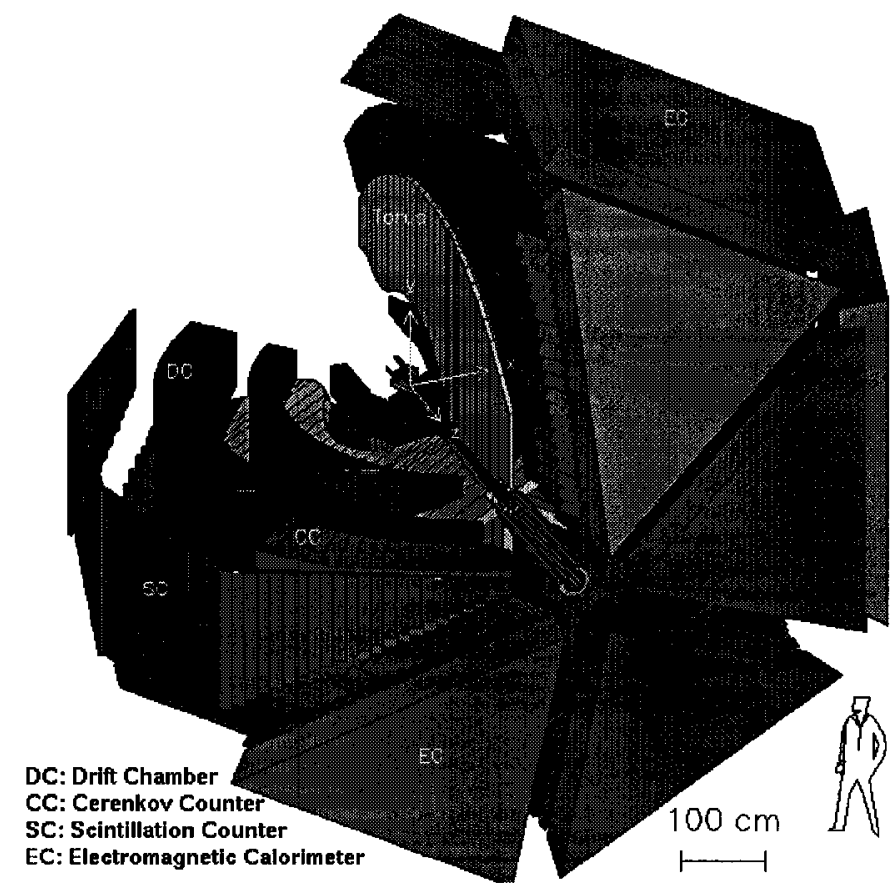

FIG. 28. A cutaway view of the CLAS. The beam direction is out of the page.

The CLAS located in experimental Hall B, has a toroidal magnetic field produced by six superconducting coils. The superconducting coils separate the detector into six wedge-shaped sectors, each of which covers approximately $60^{\circ}$ of the sphere. Each 
sector contains Drift Chambers (DC) for tracking charged particles, Cerenkov Counters (CC) to separate electrons from pions, Scintillation Counters (SC) for determining particle flight time and Electromagnetic Calorimeters (EC) to identify electrons and neutral particles. The CLAS is designed to detect particles in the range $8^{\circ}$ to $140^{\circ}$ in the polar angle with up to $80 \%$ coverage in the azimuthal angle. Because of its large acceptance CLAS is well suited for experiments that require detection of multi particle final states and also can be used to run several experiments at the same time. Fig. 28 illustrates the overall layout of the detector. In this section a brief overview of each of these CLAS subsystems will be discussed.

\subsubsection{The Torus Magnet}

The torus magnet consists of six superconducting coils arranged around the electron beam line. It generates a magnetic field, with main field components in the $\phi$ direction, that circles the beam line. This magnetic field allows one to measure the particle's momentum by inducing a curvature, which depends on the charge and the momentum of the particle, on its path. In addition to this, the cryostat holding the coils also serves as a support structure to hold the other detector packages around the beam line.

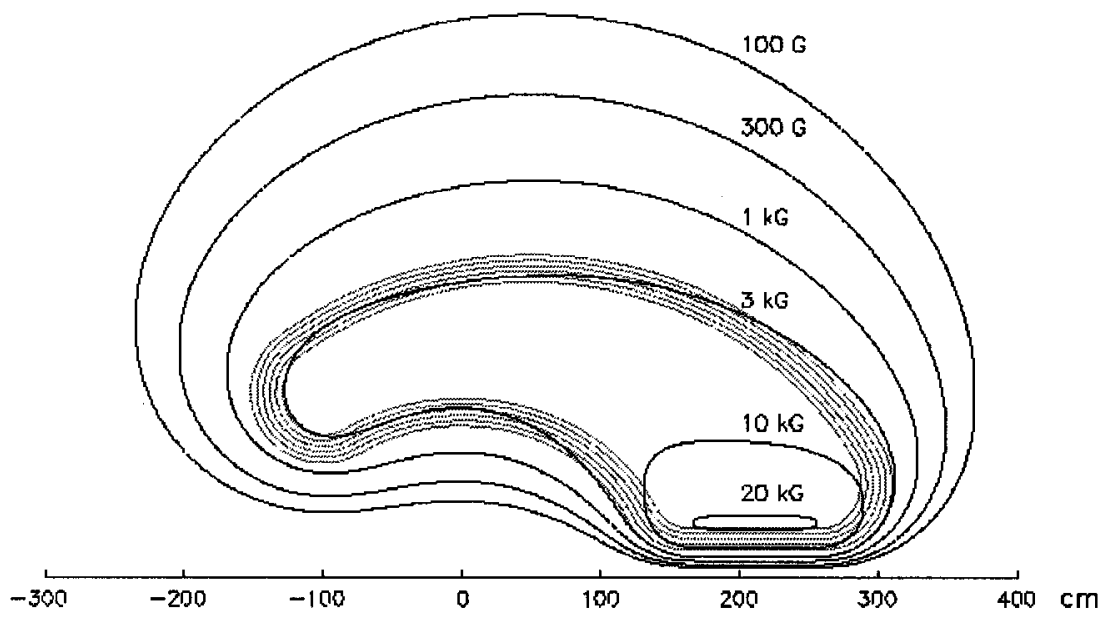

FIG. 29. Constant magnetic field contours for the CLAS torus at a current setting of $3860 \mathrm{~A}$ [41]. 


\subsubsection{Drift Chambers}

The drift chambers (DC) [42] in each sector are separated into three regions. Region one is in a low magnetic field and is the closest to the target. Region two is situated in between the torus magnet coils. Region three is the largest and is located outside the magnet coils. The relative position of the three regions are shown in Fig. 30.

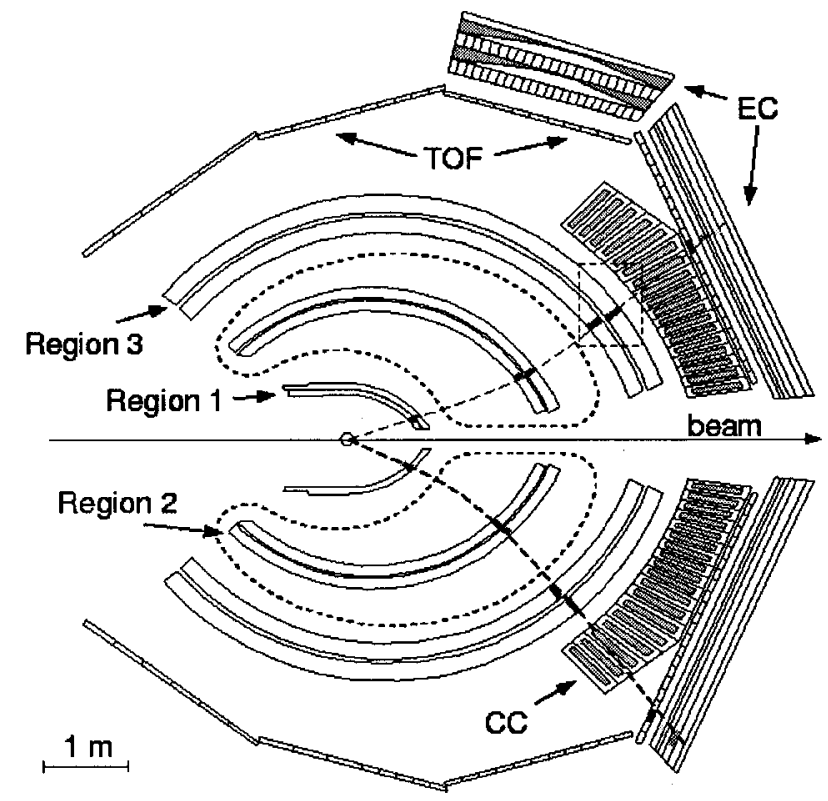

FIG. 30. Midplane slice of the CLAS, showing the region one, two and three DCs and other detectors [42].

Each region has two superlayers. A single superlayer consists of six layers of drift cells, except for superlayer one which has only four layers. A charged particle originating from the target would therefore cross 34 layers of drift cells. Each cell has six field (cathode) wires surrounding one sense (anode) wire and has a hexagonal shape. The hexagonal shape has been chosen since it provides a good approximation to the circular cell in which the drift time to drift distance conversion shows minimal dependence on the entrance angle of a particle track. The cell size (radius) increases uniformly from region 1 to region 3 and is $0.7 \mathrm{~cm}$ in region $1,1.5 \mathrm{~cm}$ in region 2 and $2.0 \mathrm{~cm}$ in region 3 .

The three regions are constructed of wires that connect to the endplates, which face each other at a $60^{\circ}$ angle, in each sector as shown in Fig. 31.

The drift cells were constructed from $20 \mu \mathrm{m}$ gold plated tungsten sense wires and 
$140 \mu \mathrm{m}$ aluminum field wires. All six sectors are filled with $88 \%$ argon and $12 \%$ carbon dioxide gas mixtures. The field wires are kept at a negative potential and the sense wires are kept at a positive potential through a high voltage system.

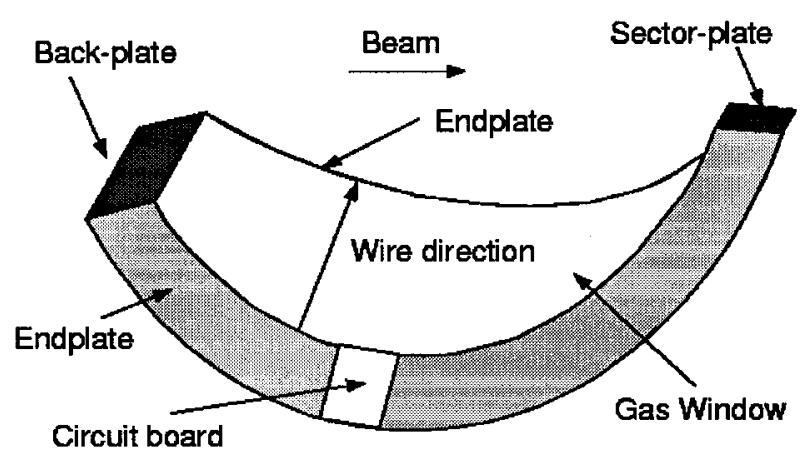

FIG. 31. One drift chamber sector showing the wire direction. The beam is from left to right.

When a charged particle goes through CLAS it ionizes the gas and produces electron ion pairs. Electrons drift to the sense wire along the field lines and produce a voltage pulse on the sense wire. The signal size depends on the operating voltage, the type of gas used, and other effects including the specific ionization of the particle track through the chamber.

\subsubsection{Time of Flight Counters}

In order to determine the mass of a charged particle the velocity of the particle has to be known, in addition to the momentum determined by the DC tracking. The CLAS Time of Flight (TOF) system [43] provides a high resolution timing measurement that can be used to calculate the mass of a charged particle. The CLAS Time of Flight counters consist of 57 scintillators per sector, of which the last 18 are paired into nine logical counters ; they are all connected to a photomultiplier tube and a light guide at each end. Within a sector these scintillators are mounted in four panels as shown in Fig. 32. The "forward-angle" counters, which are the counters $1-23$, cover out to $45^{\circ}$ and are $15 \mathrm{~cm}$ wide. The remaining scintillators, large-angle scintillators, are $22 \mathrm{~cm}$ wide. These specific widths were selected to optimize the timing resolution of a single counter. The length of the counters varies from $30 \mathrm{~cm}$ to $450 \mathrm{~cm}$. Each scintillator, which has a thickness of $5.08 \mathrm{~cm}$, is positioned in a way 


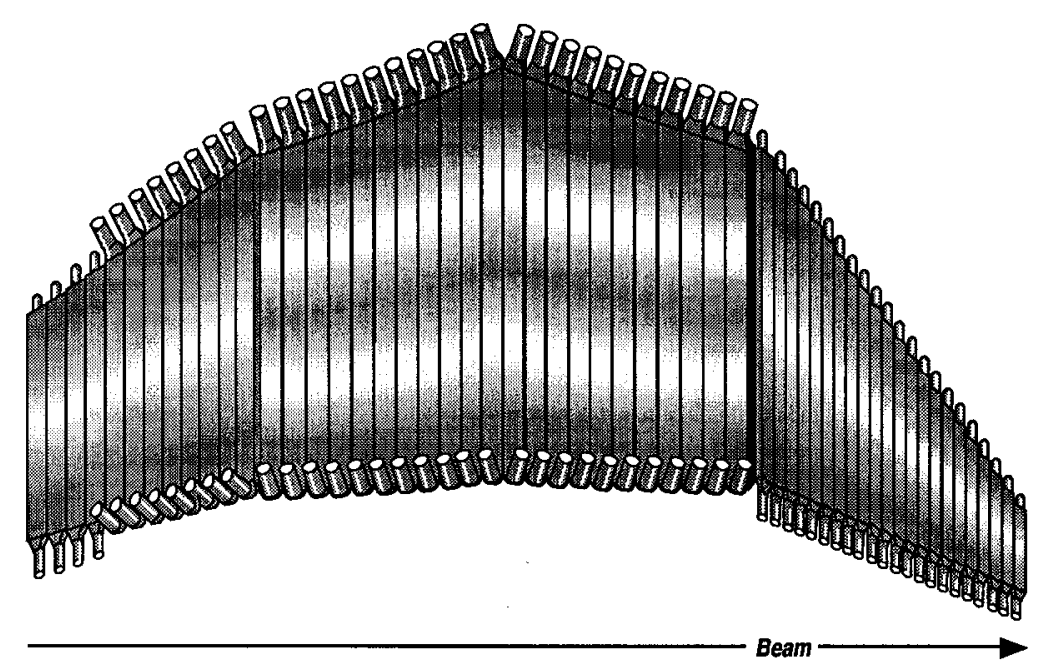

FIG. 32. Time of flight counters for one sector. It is built in 4 panels which fits the CLAS geometry [43].

that it is perpendicular to a given particle trajectory. Located approximately $5 \mathrm{~m}$ from the target, the SC are designed to fit in between the Cerenkov counters and the calorimeter and cover from $8^{\circ}$ to $142^{\circ}$ in the polar angle and $100 \%$ of the azimuthal angle. The timing resolution of each counter depends on its length. The forward counters have a better resolution than the large angle counters.

\subsubsection{Cerenkov Counters}

The CLAS Cerenkov Counters (CC) [44], which are positioned between the Drift Chamber region 3 and the Time of Flight counters, serve as part of the electron trigger and used to separate pions from electrons. It covers the region of $\theta$ up to $45^{\circ}$ in the forward direction. This was accomplished by placing light collecting cones and photomultiplier tubes in the shadow of the torus magnet and filling much of the available space with mirrors. Each sector is divided into 18 optical segments. Each of these segments is again divided into two modules along the symmetry plane of each sector, forming 36 optical modules per sector. Each of the modules is designed to focus the light in the $\phi$ direction to preserve the information of the polar angle.

Each module consists of an elliptical, a hyperbolic, and a cylindrical mirror for focusing and a "Winston" light collection cone to direct light into the photomultiplier tube. Since the photomultipliers are located in the fringe field region of the torus magnet, each PMT is shielded by a magnetic field, the magnitude of which varies 
as a function of PMT position reaching $70 \mathrm{G}$ at large $\theta$ angles. Since the Cerenkov counter is in front of the time of flight detectors and the electromagnetic calorimeters, it was necessary to keep the weight of the total material used in the construction of the CC as small as possible. This was accomplished by using layered Kevlar and vinyl foam structures for mirror support. The physics behind the CC lies in the fact

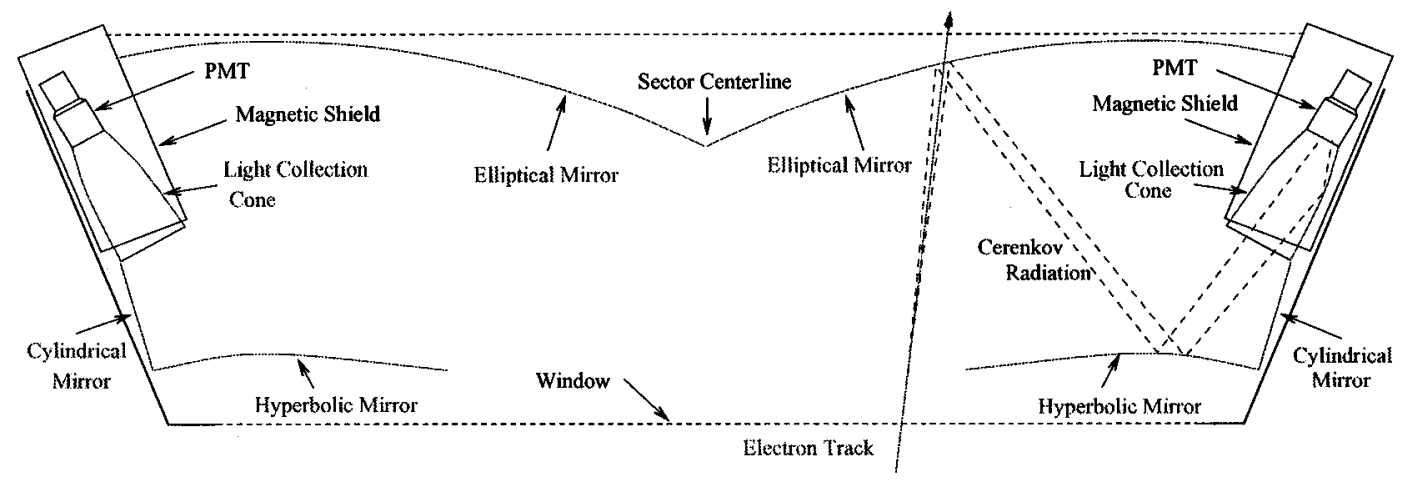

FIG. 33. A schematic of a Cerenkov segment. An example of an electron track and how the light produced is collected by the light collection cone is also shown [41].

that if a charged particle traveling in a medium exceeds the speed of light in that medium it will produce Cerenkov light. Therefore for a medium with a refraction index $n$, a particle will emit Cerenkov light if it has a velocity,

$$
v>\frac{c}{n}
$$

This Cerenkov light is collected by photomultiplier tubes and provides a signal. The medium inside the $\mathrm{CC}$ was chosen such that only charged particles which have a mass close to the electron mass will be fast enough to produce Cerenkov light. A gas that matches the above criteria, perfluorobutane $\left(\mathrm{C}_{4} \mathrm{~F}_{10}\right)$, was selected to fill the Cerenkov detector. It has an index of refraction of 1.00153 at nominal pressure and temperature and has excellent light transmission properties. This gas separates electrons from pions up to a pion momentum of about $2.7 \mathrm{GeV}$. However there is a small percentage, approximately $1 \%$, of pions that generate Cerenkov light due to primary and secondary ionization of the atomic electrons in the gas and structural elements even below a momentum of $2.7 \mathrm{GeV}$.

\subsubsection{Electromagnetic Calorimeter}

The Electromagnetic Calorimeter (EC) [45] is part of the electron trigger. In addition it can also detect neutral particles such as photons at energies above $0.2 \mathrm{GeV}$, which 


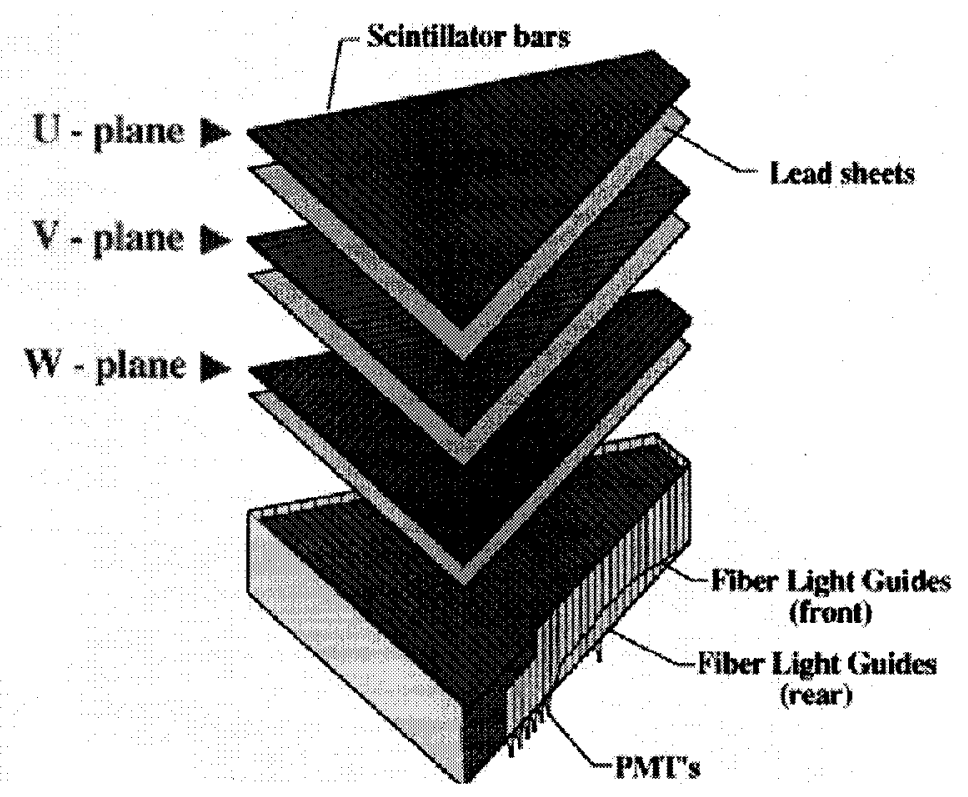

FIG. 34. Exploded view of CLAS electromagnetic calorimeter module for one sector [45].

enables the reconstruction of $\pi_{0}$ and $\eta$ from measurement of $2 \gamma$ decays, and it detects neutrons. The electromagnetic calorimeter covers a polar angles from $8^{\circ}$ to $45^{\circ}$ and $80 \%$ of the azimuthal angle. Each sector is equipped with a calorimeter which has a total thickness of 16 radiation lengths. Each calorimeter is made of alternating layers of $10 \mathrm{~mm}$ thick scintillator strips and $2.2 \mathrm{~mm}$ thick lead sheets. To fit to the hexagonal geometry of the CLAS each of these calorimeter modules was constructed in the shape of an equilateral triangle. Each of the scintillator layers is divided into 36 strips parallel to one of the edges of the triangle. The successive scintillator layers are rotated by $120^{\circ}$ to form the U, V and $\mathrm{W}$ plane as shown in Fig. 34. This helps us to determine the location of the energy deposition. Each calorimeter, which contains 13 layers, is further subdivided into an inner stack which has 5 layers and an outer stack which has 8 layers. A single photomultiplier is connected through a fiber optic cable to a single strip (1-36), plane (U, V, W) and a stack (inner, outer). Therefore, in total each calorimeter has 216 photomultiplier tubes.

The basic principle behind the EM calorimeter lies in the manner in which particles deposit energy inside the detector material. When a particle hits the calorimeter 
material it deposits energy in the material which leads to light emission in the scintillators, collected by the photomultiplier tubes. There are two primary means of depositing energy in the calorimeter, ionization and radiation. Most electrons lose all their energy through radiation and subsequent showering of $\mathrm{e}^{+} \mathrm{e}^{-}$pairs, while heavier particles, such as pions, are minimum ionizing and deposit much less energy $\left(2 \mathrm{MeV} / \mathrm{g} / \mathrm{cm}^{3}\right)$. This unique characteristic of the EC helps in subsequent particle identification. In general, about $27 \%$ of the energy deposited in the EC is detected by the scintillators, the rest is absorbed by the layers of lead.

\subsection{POLARIZED TARGET}

The polarized target materials used in the EG1 experiment were frozen $\mathrm{NH}_{3}$ and

$\mathrm{ND}_{3}$. In addition to the two polarized targets ${ }^{12} \mathrm{C},{ }^{4} \mathrm{He}$ and ${ }^{15} \mathrm{~N}$ targets were also used. The deuteron and proton nucleons were polarized using a technique called Dynamic Nuclear Polarization (DNP). The targets were placed in a $5 \mathrm{~T}$ magnetic field and $1 \mathrm{~K}$ liquid He bath. A nuclear Magnetic Resonance (NMR) system was used to monitor the polarization in addition to continuous offline calculation of target polarization using the data. In this section, an overview of the target, the theory behind DNP and how NMR was used to measure the polarization will be discussed.

\subsubsection{The Target System}

The polarized target was designed to fit inside the CLAS (Fig. 35), where the effects of the torus magnetic field are minimal. The target consisted of several subsystems,

- A pair of superconducting Helmholtz coils, which produced a uniform $5 \mathrm{~T}$ magnetic field at the location of the target cell. The magnet was kept at a temperature of $4.2 \mathrm{~K}$ through a liquid helium reservoir that was located outside the CLAS.

- A refrigeration system to keep the target material at $1 \mathrm{~K}$. This system used an evaporation refrigerator.

- A target insert. The insert shown in Fig. 36 consisted of an aluminum structure and four target cells which contained the target materials. Each cell was 10 $\mathrm{mm}$ in length and $15 \mathrm{~mm}$ in diameter. In addition each target cell was sealed using a thin aluminum foil on the entrance window and a kapton foil on the 


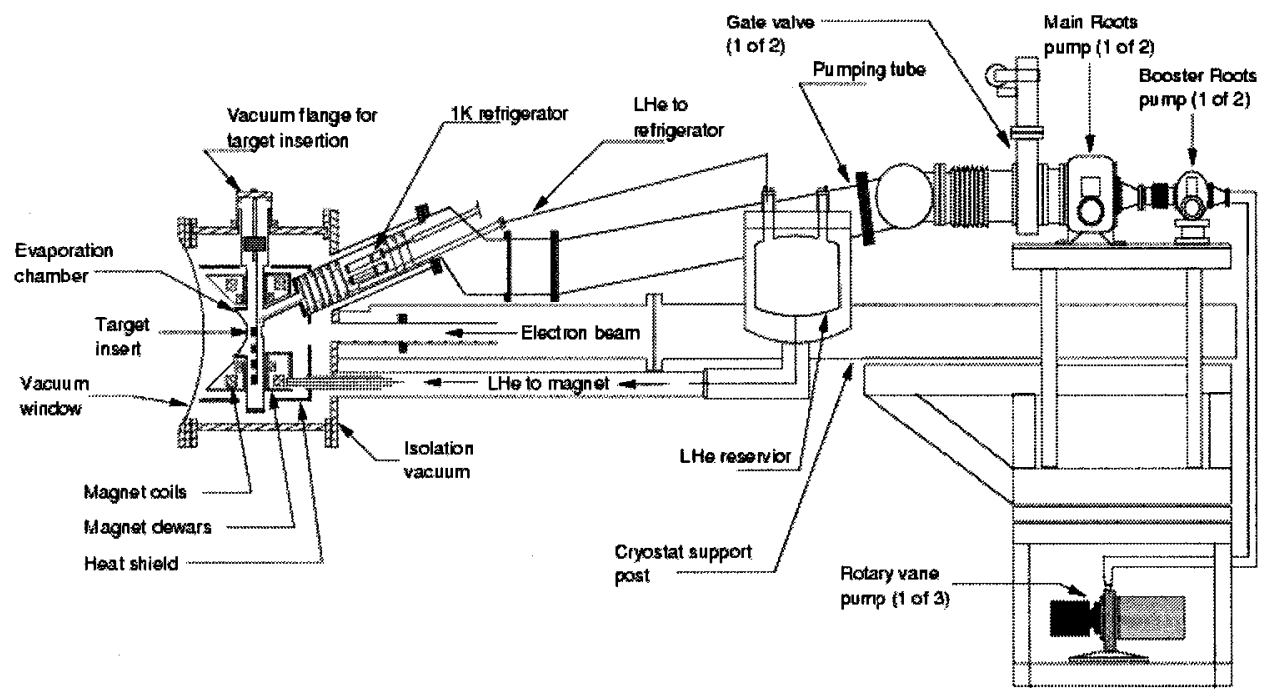

FIG. 35. A side-view of the EG1 polarized target system [46].

exit window. The insert was connected to a stepping motor, which was used to switch the targets mechanically. The NMR coils, which were used to monitor the polarization, were placed outside the $\mathrm{ND}_{3}$ and $\mathrm{NH}_{3}$ cell walls. Temperature sensors were placed on the insert to monitor the condition of the target materials.

- A NMR system. The NMR system consisted of a resonant LRC circuit. More details about the NMR measurements will be discussed in the section 3.4.4.

The entire target system was built so that scattered particles could exit in a forward cone of $50^{\circ}$ or perpendicular to the beam from $75^{\circ}$ to $105^{\circ}$ in the azimuthal angle. A more detailed overview of the EG1 target system is given in [46].

\subsubsection{Polarized Target Materials}

The polarized target materials used in the EG1 experiment were ${ }^{15} \mathrm{NH}_{3}$ and ${ }^{15} \mathrm{ND}_{3}$. These materials were chosen for several reasons,

- Both these materials have a high percentage of polarizable nucleons, $16.7 \%$ for ${ }^{15} \mathrm{NH}_{3}$ and $28.6 \%$ for ${ }^{15} \mathrm{ND}_{3}$.

- They show a high resistance to radiation damage. 


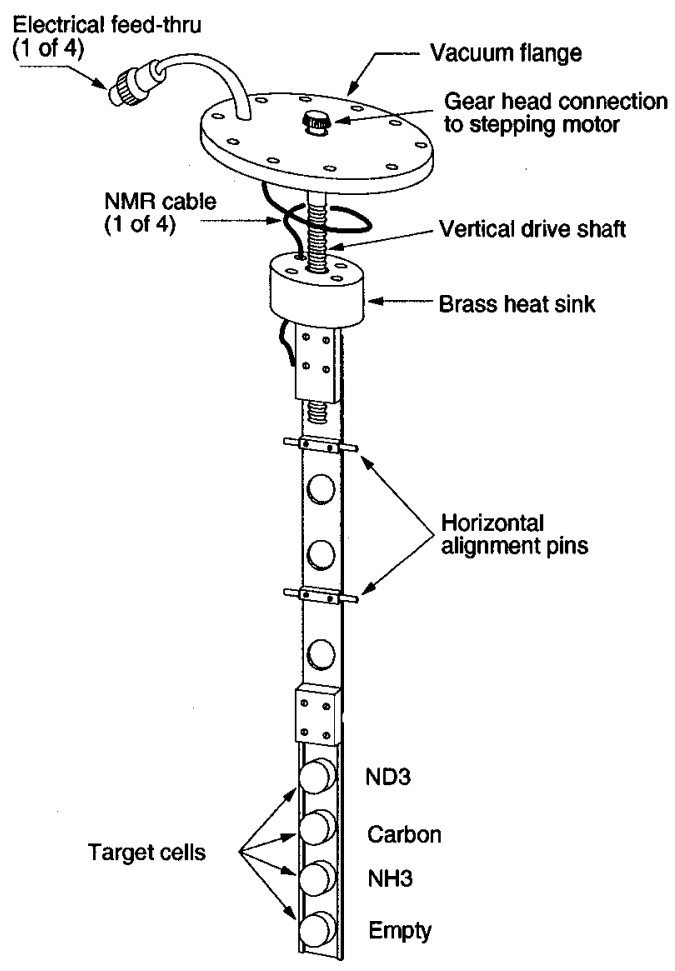

FIG. 36. A schematic of the target insert used during the EG1 run. The NMR coils are not shown [46].

- Since the spin of the ${ }^{15} \mathrm{~N}$ is carried by a single valence proton, asymmetries due to the polarization of ${ }^{15} \mathrm{~N}$ are well understood and easier to take into account.

Gaseous ammonia was frozen and then was crushed into small beads to make the target. Small beads were used to prevent overheating of the target.

\subsubsection{Dynamic Nuclear Polarization}

When a material is placed in a magnetic field unpaired electron and nuclear spins tend to align themselves in the direction of the magnetic field due to interactions between the magnetic moments, $\mu$, and the magnetic field. The magnetic moment itself depends on the spin, $S$, of the particle and for an electron it is given by,

$$
\mu=g \mu_{e} S
$$

and for a nucleon it is given by,

$$
\mu=g \mu_{N} S
$$


where $\mu_{e}$ and $\mu_{N}$ are the Bohr magneton and the nuclear magneton and the factor $g$ is the $\mathrm{g}$-factor of the particle. The polarization is a measure of this alignment of nuclear spins. For a nucleus with spin J, the nuclear polarization, $\mathrm{P}$, is given by,

$$
P_{J}=\frac{\sum_{i} J_{Z, i} N_{i}}{J \sum_{i} N_{i}}
$$

where $N_{i}$ is the total number of nuclei, with spin projection along the axis of the magnetic field. The sum is over the $2 J+1$ orientations of the spin along the direction of the magnetic field, which is considered here as the $z$ axis. The polarization $P$ depends on the magnetic field intensity, $B$, and the spin temperature of the system, $T$. Therefore the polarization of a spin $1 / 2$ particle, $P_{1 / 2}$, is given by,

$$
P_{1 / 2}=\frac{N_{1 / 2}-N_{-1 / 2}}{N_{1 / 2}+N_{-1 / 2}}=\tanh \frac{\mu B}{k T},
$$

and for a spin 1 particle, $P_{1}$, the polarization is given by,

$$
P_{1}=\frac{N_{1}-N_{-1}}{N_{1}+N_{0}+N_{-1}}=\frac{4 \tanh \frac{\mu B}{2 k T}}{3+\tanh ^{2} \frac{\mu B}{2 k T}},
$$

where $k$ is the Boltzmann's constant. For temperatures and magnetic fields that can be physically achieved, the polarization attainable for a spin 1 or a spin $1 / 2$ nucleus stays below $1 \%$. However under the same conditions the electrons can have a polarization of $99 \%$.

Dynamic Nuclear Polarization (DNP) is used to increase these low polarizations enormously. To use DNP the target material has to be doped with paramagnetic impurities, usually in the form of unpaired electrons at a relatively low concentration $\left(\sim 10^{-4}\right)$. These impurities can be included into the material either by introducing chemicals or irradiating the material. The EG1 target materials were doped using either the $20 \mathrm{MeV}$ electron beam of the Stanford University SUNSHINE facility or the $38 \mathrm{MeV}$ electron beam of the TJNAF Free Electron Laser while keeping the material at a temperature close to $80 \mathrm{~K}$. On average $10^{17}$ electrons $/ \mathrm{cm}^{2}$ were applied to the target materials.

To understand the principle behind DNP, lets consider the Hamiltonian of a system of free electrons and a spin $1 / 2$ nucleon placed in a magnetic field,

$$
H=H_{0}-\overrightarrow{\mu_{e}} \cdot \vec{B}-\overrightarrow{\mu_{N}} \cdot \vec{B}+H_{s s} .
$$

The term $H_{0}$ is the free Hamiltonian for the electrons and the nucleon. The second term describes the interaction between the electron and the magnetic field while the 
third term describes the interaction between the nucleon and the magnetic field. The final term arises due to dipole-dipole interactions between the electron and the nucleon. The effects of $H_{s s}$ on the free Hamiltonian is relatively small compared to the second and the third term. Therefore the eigen states of $H$ can first be calculated by treating $-\overrightarrow{\mu_{e}} \cdot \vec{B}-\overrightarrow{\mu_{n}} \cdot \vec{B}$ as a perturbation to the free Hamiltonian. Fig. 37 shows
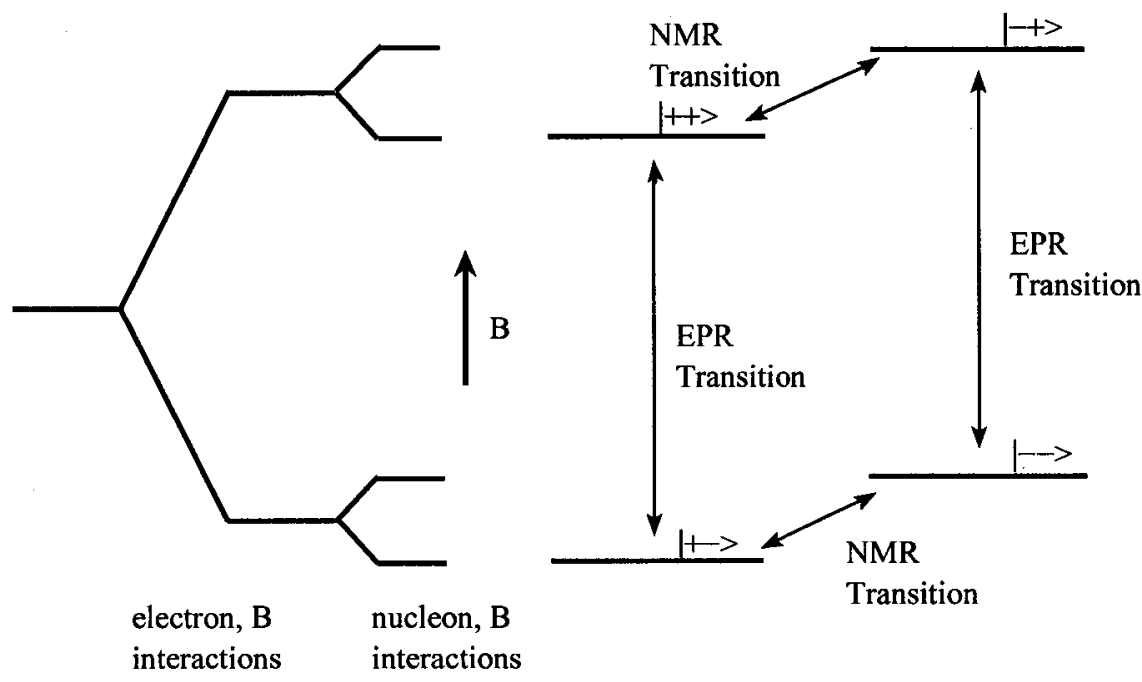

Transition

FIG. 37. The energy level diagram of a spin $1 / 2$ nucleon electron system that is placed in a magnetic field. Details are given in the text.

the splitting of energy levels, known as the Zeeman effect, due to this perturbation. There are four states: $|+-\rangle,|--\rangle,|++\rangle$ and $|-+\rangle$, where the first sign indicates the nucleon spin direction and the second sign indicates the electron spin direction. The transitions $|-+\rangle \rightarrow|++\rangle$ and $|--\rangle \rightarrow|+-\rangle$ are due to the nucleon spin flip, NMR transitions, and the transitions $|-+\rangle \rightarrow|--\rangle$ and $|++\rangle \rightarrow|+-\rangle$ are due to electron spin flip, EPR (Electron Paramagnetic Resonance) transitions. However, transitions $|++\rangle \rightarrow|--\rangle$ and $|-+\rangle \rightarrow|+-\rangle$ cannot occur since that requires flipping both the nucleon and the electron spin and are forbidden due to the dipole selection rules. The $H_{s s}$ term can be treated as a perturbation to the Hamiltonian, $H_{0}-\overrightarrow{\mu_{e}} \cdot \vec{B}-\overrightarrow{\mu_{n}} \cdot \vec{B}$. The $H_{s s}$ causes mixing of the energy levels due to the first perturbation (Fig. 38),

$$
\begin{aligned}
& |1\rangle=a|++\rangle+b|-+\rangle, \\
& |2\rangle=a|+-\rangle+b|--\rangle, \\
& |3\rangle=a|-+\rangle+b^{*}|++\rangle, \\
& |4\rangle=a|--\rangle+b^{*}|+-\rangle .
\end{aligned}
$$


However in all the above cases the term $a \approx 1$ and the term $b \approx 0$ making it very sim-

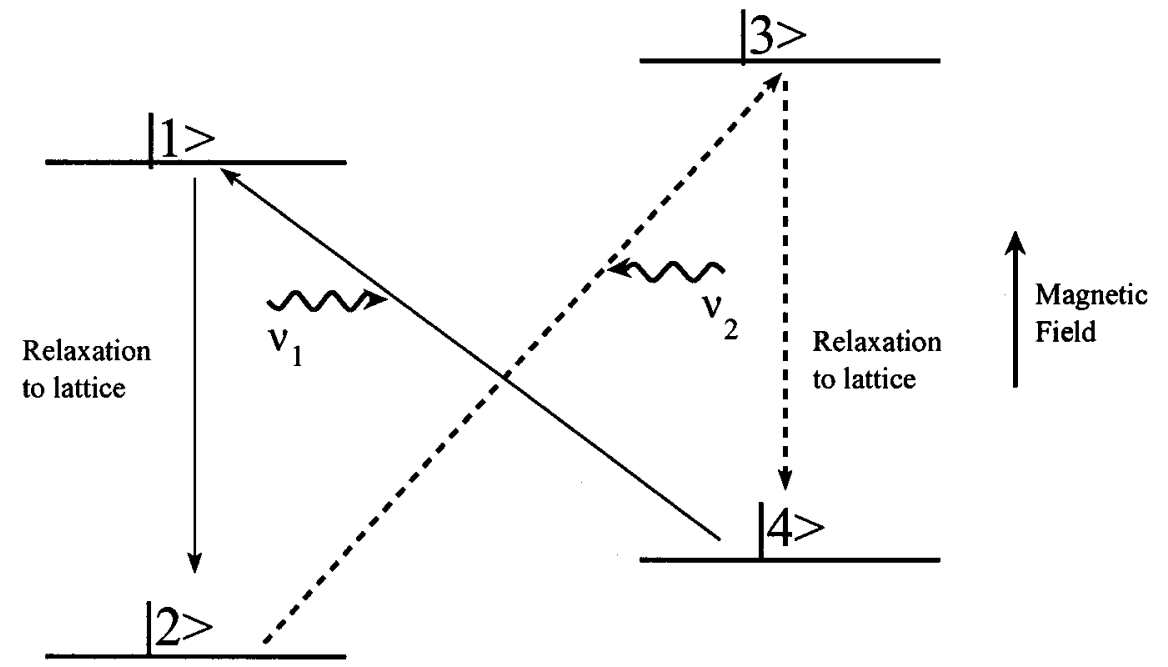

FIG. 38. The transition between energy levels that are used in DNP.

ilar to simple Zeeman splitting given in Fig. 37. If photons of the correct frequency, $\nu_{1}=\left(\mu_{e}-\mu_{n}\right) B / h$ and $\nu_{2}=\left(\mu_{e}+\mu_{n}\right) B / h$, are directed on the material the forbidden transitions $|4\rangle \rightarrow|1\rangle$ and $|2\rangle \rightarrow|3\rangle$ will occur. For the EG1 experiment microwaves were used to induce these transitions. The relaxation time for electrons is small, $\sim 10^{-3} \mathrm{~s}$, so almost all the electrons will relax back to the lower energy state (Fig. 38) very quickly and can be used to flip the spin of additional nucleons. However, since the relaxation time of the nucleons is much larger, $\sim 10^{3} \mathrm{~s}$, nucleons near a free electron will populate one spin state. The direction of the total polarization can be selected by choosing the appropriate frequency $\nu_{1}$ or $\nu_{2}$. This method of improving the nuclear polarization by transferring the high polarization of the electrons to the nucleon is known as DNP.

\subsubsection{Monitoring the Target Polarization}

A Nuclear Magnetic Resonance (NMR) system was used to monitor the polarization of the EG1 target. As described in the previous section the spin of the target nucleons were aligned in a preferred direction by applying a magnetic field $B$, which causes the energy levels to split. In addition, the spins of the nucleons precess about the direction of the applied $B$ field because the nucleon undergoes a torque of the form, $|\gamma \vec{L} \times \vec{B}|$, where $\gamma$ is the gyromagnetic ratio and $L$ is its angular momentum. The frequency of this precession, known as the Larmor frequency, is given by 


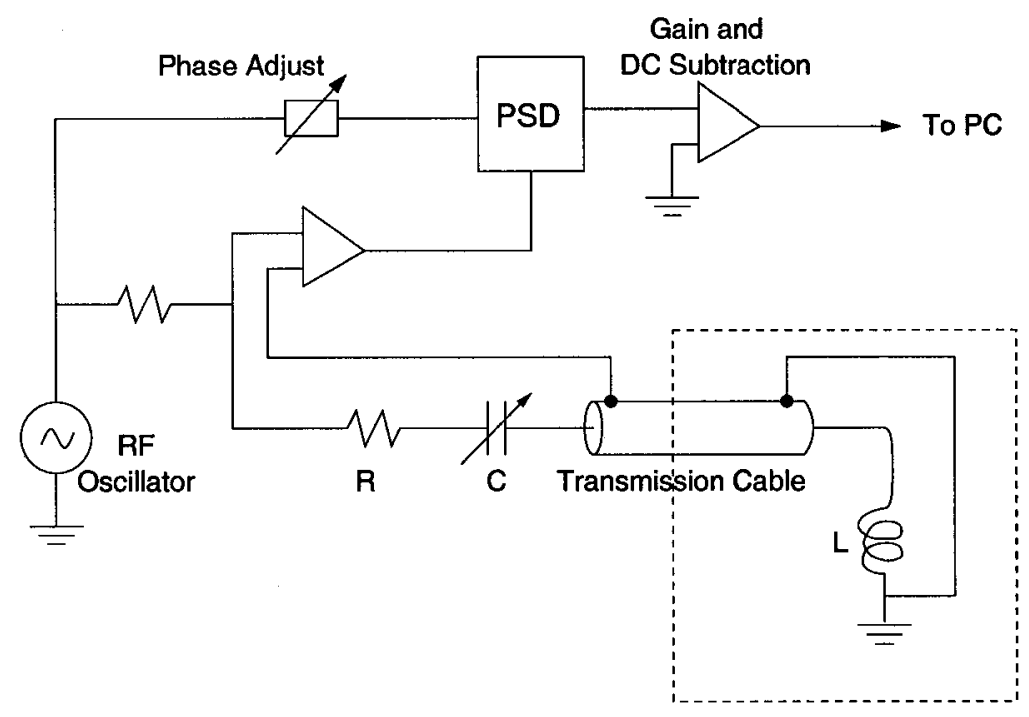

FIG. 39. A diagram of the NMR circuit [46].

$\omega_{0}=\gamma|\vec{B}|$. In the NMR technique an oscillating magnetic field $B_{1}$ (radiofrequency) of appropriate frequency $(\omega)$ and duration, perpendicular to the static $B$ field is applied. This causes the nucleons to have a spin precession along the field of $B_{1}$ at a frequency, $\omega_{1}=\gamma\left|\vec{B}_{1}\right|$. Since the system has discrete energy levels, at the frequency $\omega=\omega_{0}$ (and closely around because of the Heisenberg uncertainty and Doppler effects), the system absorbs the energy applied by the signal and flips the spin of the nucleons which in-turn affects the susceptibility of the material,

$$
\chi(\omega)=\chi^{\prime}(\omega)-i \chi^{\prime \prime}(\omega) .
$$

For a given spin type the absorptive part, $\chi^{\prime \prime}(\omega)$, of the susceptibility is sensitive to the polarization of the material [47],

$$
P \propto \int_{0}^{\infty} \chi^{\prime \prime}(\omega) d \omega .
$$

In the EG1 experiment the NMR system shown in Fig. 39 was designed to apply the required magnetic field and to measure the susceptibility of the target material, thereby measuring the polarization. A wire coil was placed around the target to apply the oscillating magnetic field $B_{1}$. A radiofrequency near the Larmor frequency was applied to flip the spin of a small fraction of the nucleons. This causes the susceptibility of the material to change. The coil is also used as an inductor in an alternating current LRC circuit. The circuit can be used to measure the susceptibility 
of the sample since the inductance of the coil depends on the susceptibility of the material which surrounds the coils,

$$
L(\omega)=L_{0}(1+4 \pi \eta \chi(\omega)),
$$

where $L_{0}$ is the inductance of the coil when the material is completely unpolarized and the filling factor $\eta$ is a parameter describing the coupling of the material to the coil. The susceptibility, which is required to measure the polarization, is observed as a change in the complex impedance of the circuit. The RF frequency is swept

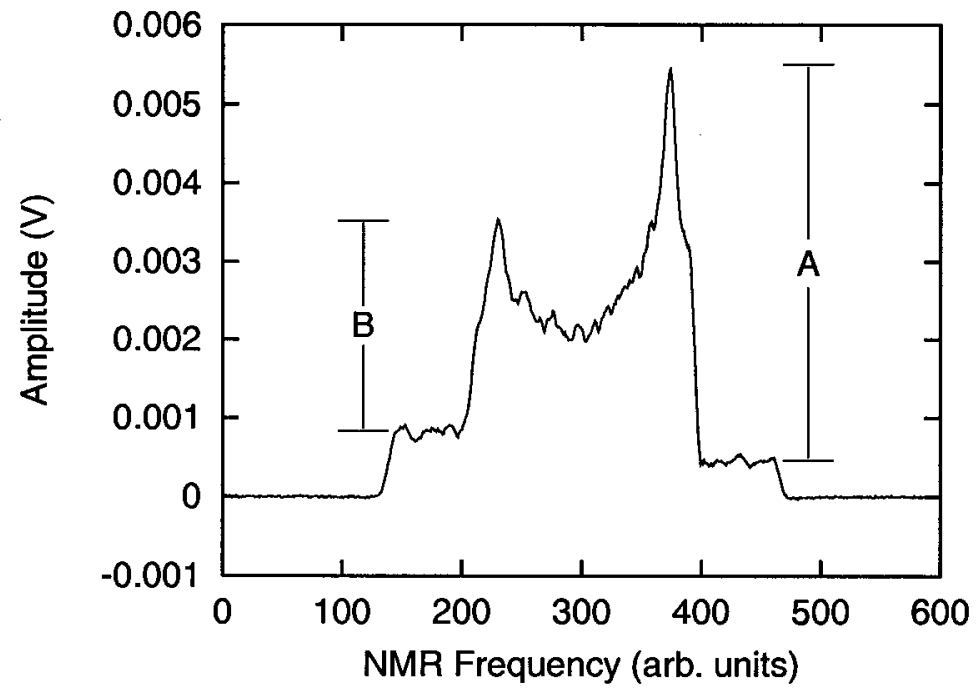

FIG. 40. An enhanced polarization NMR signal for $\mathrm{ND}_{3}$. Details are in the text [46].

through a range of frequencies above and below the larmor frequency to obtain the NMR signal. The area under the curve is proportional to the polarization of the target. To accurately determine the polarization it is also necessary to remove the factor that depends on the circuit and sample parameters. Therefore another measurement at thermal equilibrium (i.e., no beam, microwaves were off so that there was a uniform, known temperature in the target cell), where the polarization is known, was made to properly correct those effects. Fig. 40 shows the corrected $\mathrm{NMR}$ signal for $\mathrm{ND}_{3}$. Since $\mathrm{ND}_{3}$ is a spin 1 particle, in an external magnetic field it has 3 magnetic substates. The deuterium has a significant quadrupole moment which couples to the electric field gradient created from the atomic electrons in the $\mathrm{ND}_{3}$ lattice to distort the Zeeman states. The energy spacing between the 3 states is therefore not uniform and there are two NMR transitions with two frequencies. The two peaks correspond to these two frequencies. The polarization of the deuteron can 
be measured either as the area under the curve or using the relative heights of the two peaks, $P=\left(r^{2}-1\right) /\left(r^{2}+r+1\right)$, where $r=A / B$. Fig. 41 shows some of NMR measurements taken during the EG1 run period.

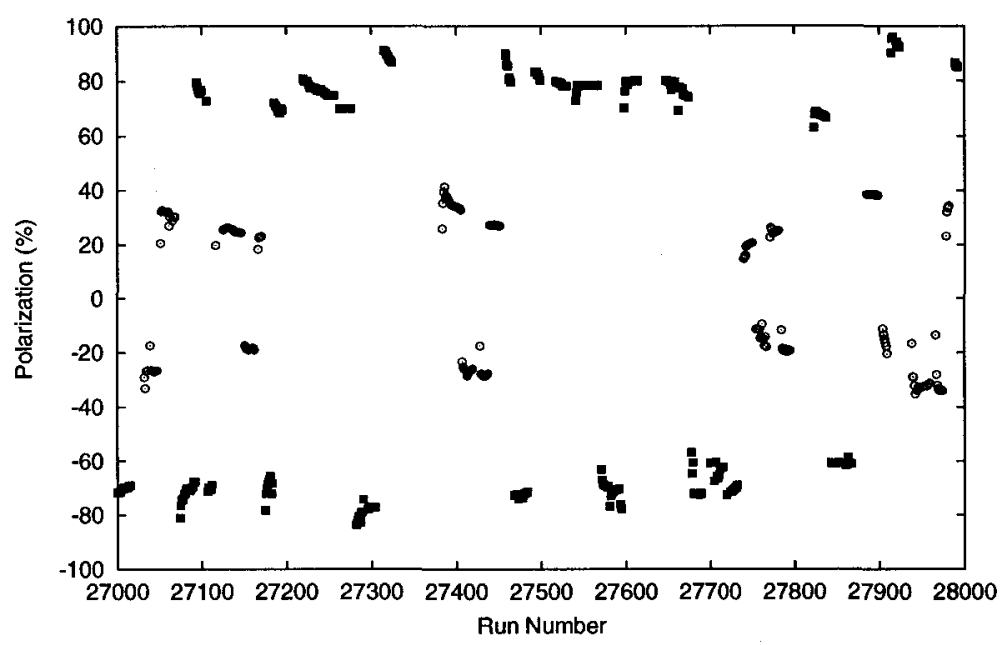

FIG. 41. Polarization measured by the NMR for different runs [46]. Filled squares correspond to the $\mathrm{NH}_{3}$ measurements and $\odot$ correspond to $\mathrm{ND}_{3}$ measurements.

\subsection{THE TRIGGER AND THE DATA ACQUISITION SYSTEM}

The CLAS trigger system is built around a two level system, the level-1 and the level-2 trigger. The level one trigger requires a signal above threshold in the $\mathrm{CC}$ and EC (the TOF can be included in the trigger). If an event passes through the level one trigger then the level-2 trigger selects events by using the DC track hits and by identifying a preliminary track. Selecting a proper trigger is necessary to maximize the number of good events recorded and to minimize the background hits. It also reduces the unnecessary offline processing time of background events.

For the EG1 experiment, only the level one trigger was used. The level one settings required a hit in coincidence in the $\mathrm{CC} * \mathrm{EC}_{\text {inner }} * \mathrm{EC}_{\text {total }}$. The thresholds for each beam and torus setting were selected appropriately. The trigger thresholds of different EG1 data sets can be found at [48]. A complete overview of the CLAS trigger system is given in [41].

For events that pass the trigger requirements of the experiment energy and timing information for all detectors with a signal above the specification threshold are digitized in 24 FASTBUS and VME crates and are collected by 24 VME Readout 


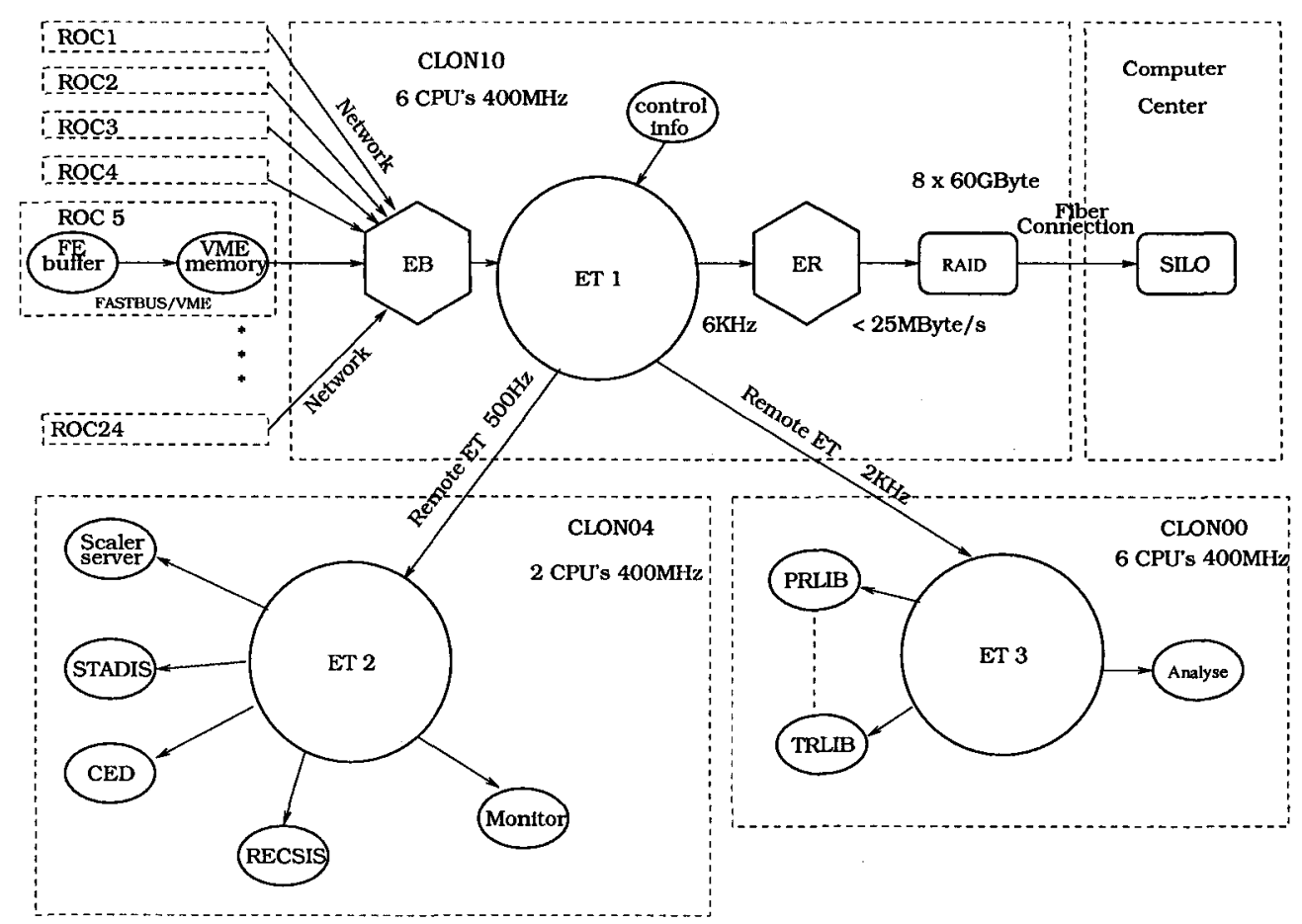

FIG. 42. A schematic of the CLAS data flow.

Controllers. These digitized data are then translated into tables and transferred through fast Ethernet lines to the online data acquisition system. The data acquisition system performs three important functions (Fig. 42):

- Event Builder (EB) links the different detector information together and builds a complete event. These events are organized in a predefined arrays called BOS banks and an event number and a run number for each event is assigned.

- The EB transfers the completed events to the Event Transport (ET). Some of the ET systems are used for temporary storage of build data to perform online monitoring, such as online analysis, event displays and detector performance.

- The Event Recorder (ER) performs the final task of data acquisition, permanent storage of data. First the data are written into a disk and then are transferred to tape that is located in the control room of the computer center.

The accelerator, the CLAS detector and the EG1 polarized target together provided an excellent combination of resources to collect data on polarized electrons scattered off of polarized nucleons. The well controlled beam and target polarization 
permitted us to study the spin structure of the nucleon. In the next chapter the first step towards the analysis and extraction of results, the event reconstruction, will be described. 


\section{CHAPTER 4}

\section{EVENT RECONSTRUCTION}

One of the important features of the CLAS is its ability to distinguish between different particles enabling the study of multi particle final states. The identification of different particles depends on the resolution of each detector which in-turn depends on the proper calibration of each detector mentioned in the previous chapter. The standard procedure is to calibrate each detector separately, obtaining reasonably good calibration constants, evaluate the quality of the processed data and do another round of calibrations if necessary. The calibration constants of each data set are saved in a data base, which subsequently will be used by the reconstruction software. The reconstruction software combines all the information together to identify the tracks and the relevant information for the event. The reconstruction of events consists of identification of charged and neutral particles along with their momenta. Identification of the trigger particle, the electron, requires a matching track in $\mathrm{DC}$, $\mathrm{CC}, \mathrm{EC}$ and TOF counters. In this chapter the procedure by which information from different detectors is combined to identify an event and the important role detector calibrations play in achieving this goal will be discussed.

\subsection{TRACK RECONSTRUCTION}

The resolution of each detector is ultimately related to the final track reconstructed by the reconstruction software. Therefore calibration of detectors and the track reconstruction cannot be treated as two different processes. More detailed instructions on how the CLAS detectors are calibrated are given in references [49], [50] and [51]. The main purpose of this section is to give a brief overview of how an event is reconstructed from raw data, TDC and ADC values, and the quality of the reconstructed EG1 data that were included in this thesis.

\subsubsection{Finding a Particle Track}

To find a good track, matching hits in time and position in the relevant detector components must be identified. The manner in which a track is identified is different for charged and neutral particles. The following section gives a brief description of how the reconstruction software identifies a hit and a possible track in CLAS for 
both charged and neutral particles.

\section{Charged particles}

- DC

The first stage of track reconstruction only uses the hit position to identify a possible particle track. The procedure, which is called hit based tracking (HBT), is based on a series of pattern finding algorithms. A possible charged particle track in the DC is first identified as follows [41],

1. First a cluster of hits in each superlayer is identified.

2. Then a lookup table is used to find a group of hits that form a track in a given superlayer.

3. Finally all segments of each superlayer are linked, using a lookup table to identify a possible track.

- $\mathrm{CC}$

To identify a hit in the CC that matches a hit in other detectors, the difference in polar angle between the track projected to the CC plane and the detected position of the hit in the CC should be less than $12^{\circ}$. Information related to the azimuthal hit position is not available for $\mathrm{CC}$ due to the manner in which the mirrors are oriented. Therefore for the $\mathrm{CC}$ only polar hit position can be matched.

- SC

To identify a hit in the TOF counter and a possible track for that hit, the distance between the $\mathrm{z}$ position of the projected track and the $\mathrm{z}$ position of the detected hit on the TOF scintillation counter should be within $30 \mathrm{~cm}$ and no information on the azimuthal position is matched.

- EC

To find a hit in the EM calorimeter, groups of strips in each U, V and W plane are first identified (Fig. 43). Then the groups are sorted according to the total energy for all strips and a centroid is calculated for each view. Finally all the information is combined to try to identify a possible match. Because of the unique geometry of the calorimeter a match can be found by requiring 
a constant for the sum of $\mathrm{U}, \mathrm{V}$ and $\mathrm{W}$ peak positions (triangle sum rule). To identify a track that agrees with the other detector components a software cut of $30 \mathrm{~cm}$ between the identified hit position and the projected track is applied.

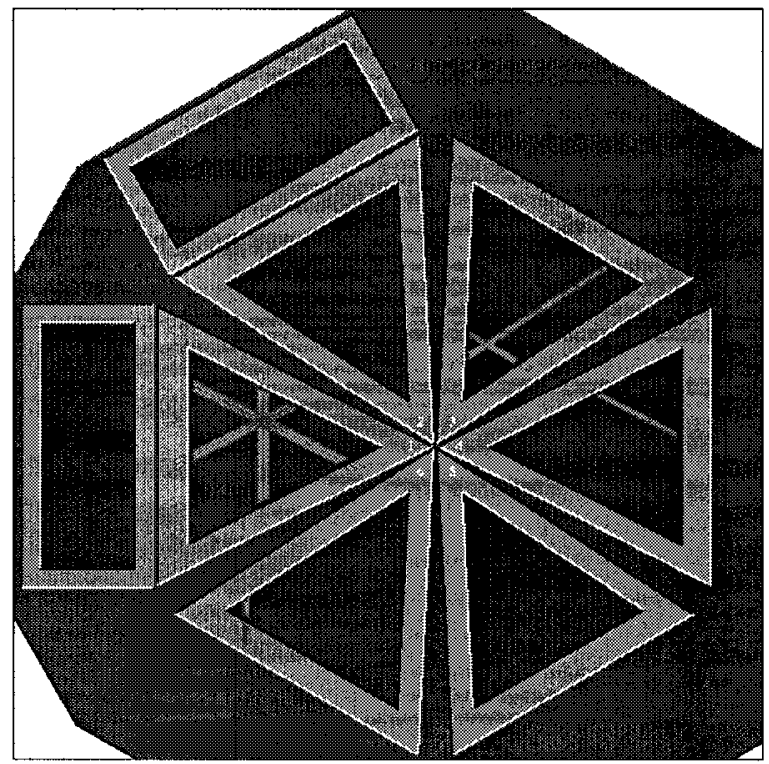

FIG. 43. A hit in the EC. The U, V and $\mathrm{W}$ view for the hit in sector 1 can be seen.

\section{Neutral particles}

Neutral particles are detected either in the EC, SC or both. The neutral particles are identified by finding clusters in the outer detectors with no charged particle track. The clusters are identified by determining their energy, the position and the time of the hit. Since photons only deposit energy in the calorimeter, $\pi^{0}$ and $\eta$ mesons are identified by detecting two photon hits in coincidence in the EC and constructing the mass $\left(M_{2 \gamma}\right)$ (Fig. 44 ) of the primary particle that corresponds to the $2 \gamma$ decay using the measured energy $\left(E_{\gamma 1,2}\right)$ and the polar angle $\left(\theta_{\gamma 1,2}\right)$ of each photon [45],

$$
M_{2 \gamma}=2 E_{\gamma 1} E_{\gamma 2}\left(1-\cos \left(\theta_{\gamma 1}+\theta_{\gamma 2}\right)\right)
$$

The resolution of the identified particles depends on the measured energy and the angle of the identified photon.

The neutrons are detected by identifying a hit in the calorimeter that does not satisfy any of the requirements of a charged particle. Neutrons are distinguished from photons by their flight time to the EC. The neutron detection efficiency increases with 
the momentum of the neutron, from $5 \%$ for $0.6 \mathrm{GeV}$ neutrons to about $50 \%$ above 2 $\mathrm{GeV}$.

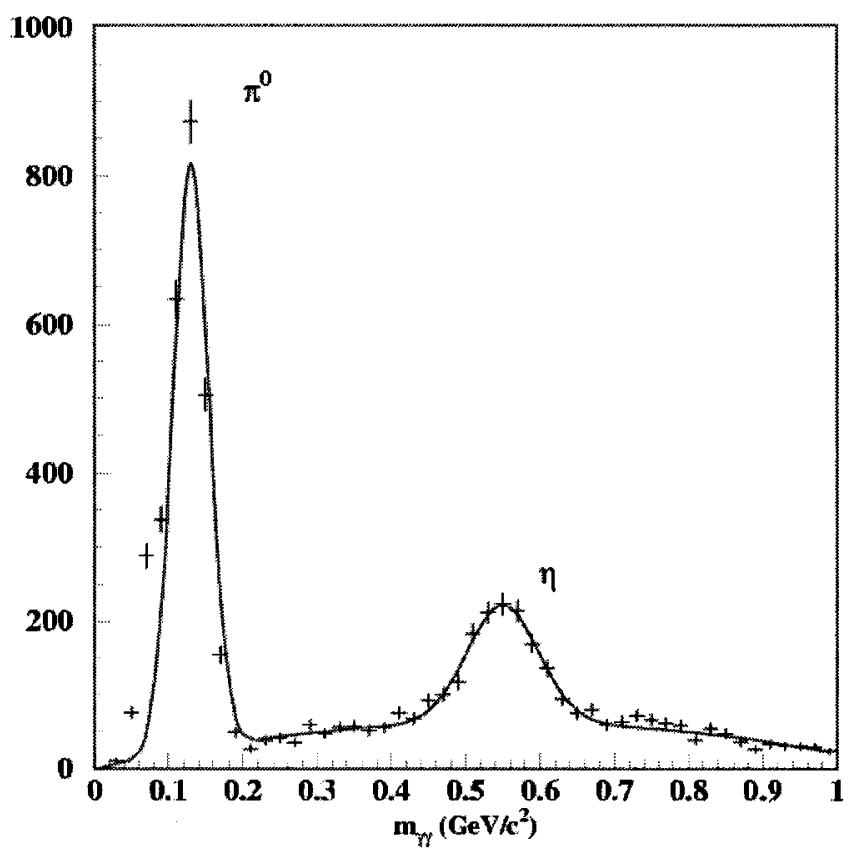

FIG. 44. Invariant mass of $\pi^{0}$ and $\eta$, reconstructed using two photon events recorded in the $\mathrm{EC}[41]$.

\subsubsection{Reconstruction of the Start Time}

One of the most important things in the reconstruction of a track is to find its start time. The start time of an identified track is determined using the time of flight counter. The calibration of the time of flight counter includes converting the raw time-to-digital (TDC) and amplitude-to-digital (ADC) values to time and energy respectively. In order to do this, any additional time delays caused by the leading edge discriminator on the signal amplitude, the time walk correction, must also be taken into account. After all these are properly calibrated, the time delays for individual scintillators with respect to each other are then adjusted. This is done by using the RF-signal from the accelerator as the reference timing signal. As previously mentioned, the beam is delivered to Hall-B in bunches with a frequency of $499 \mathrm{~Hz}$, which corresponds to a time interval of $2.0039 \mathrm{~ns}(\Delta T)$ between two separate bunches. Since all electron bunches sent to the hall are separated by the same time interval, it can be used to align the timing of all scintillation counters to the same RF bunch. 
Once all the individual channels are calibrated, the start time $\left(T_{\text {start }}^{e l}\right)$ of the trigger electron can be calculated using,

$$
T_{\text {start }}^{e l}=T_{S C}^{e l}-\frac{l}{\beta_{e l} c}-T_{R F \text { off }}
$$

where $T_{S C}^{e l}$ is the time recorded at the TOF counter and $c$ is the speed of light. The term $l / \beta_{e l} c$ calculates the start time using the total length of the electron track by tracing it to the vertex and assuming it is traveling at the speed of light, that is by assuming $\beta_{e l}=1$. The time $T_{R F o f f}$ is given by,

$$
T_{R F o f f}=\bmod \left(\left(T_{S C}^{e l}-\frac{l_{e l}}{\beta_{e l} c}-t_{R F}\right), \Delta T\right)-\frac{\Delta T}{2},
$$

where $t_{R F}$ is the RF time. Since the RF time is sent to the hall with a prescale factor of 40 it is not possible to identify which bunch contained the trigger electron. Therefore it is necessary to divide the difference between the start time calculated using only the TOF information, $T_{S C}^{e l}-\frac{l_{e l}}{\beta_{e l} c}$ and the RF time of that particular bunch $\left(t_{R F}\right)$ by $\Delta T$ and to take the remainder (Fig. 45). The calibration of each individual

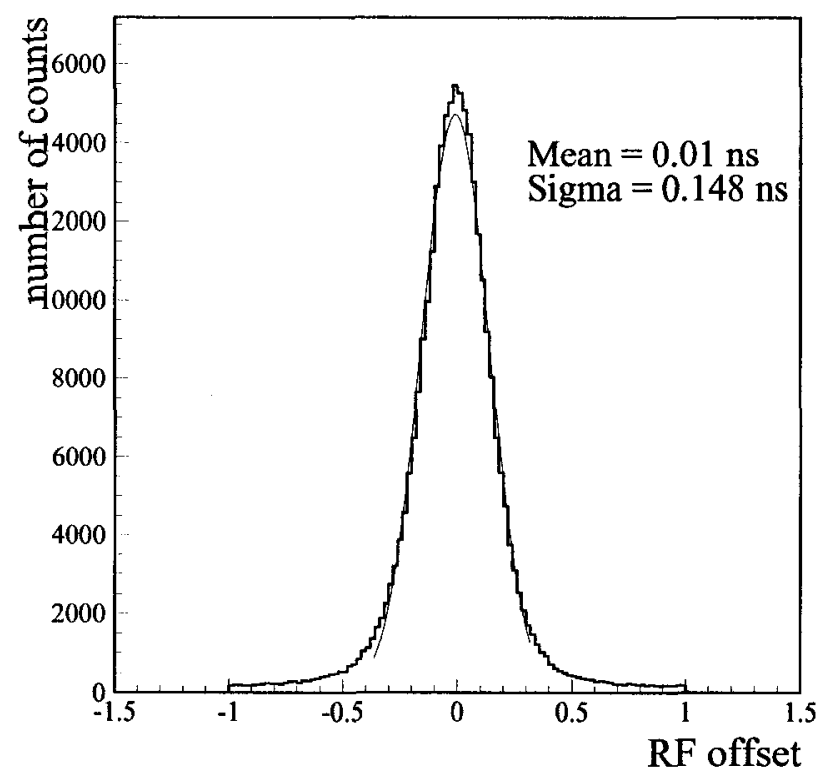

FIG. 45. The RF offset for one EG1 run. Data are taken at a beam energy of 1.6 $\mathrm{GeV}$.

channel also depends on the calibration of the RF-signal itself. Miscalibration of the RF time will be reflect in the RF offset which should look like Fig. 46 when properly calibrated. Once all different aspects of the TOF calibration are taken into 


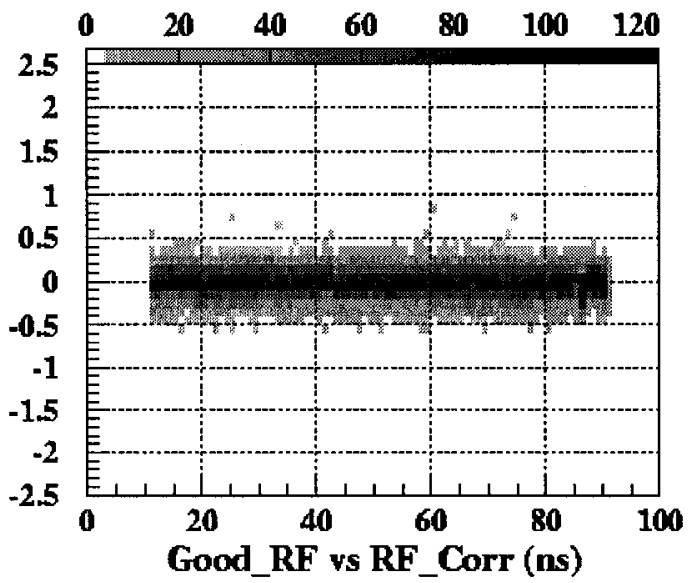

FIG. 46. The RF offset vs RF time should not show any RF dependence when the TOF is properly calibrated.

account the RF offset distribution should be peaked around zero and should have a sigma in the range of 150 to $200 \mathrm{ps}$ (Fig. 45). The RF offset is the primary means of measuring the resolution of the TOF counter (Fig. 47). The TOF beta of other

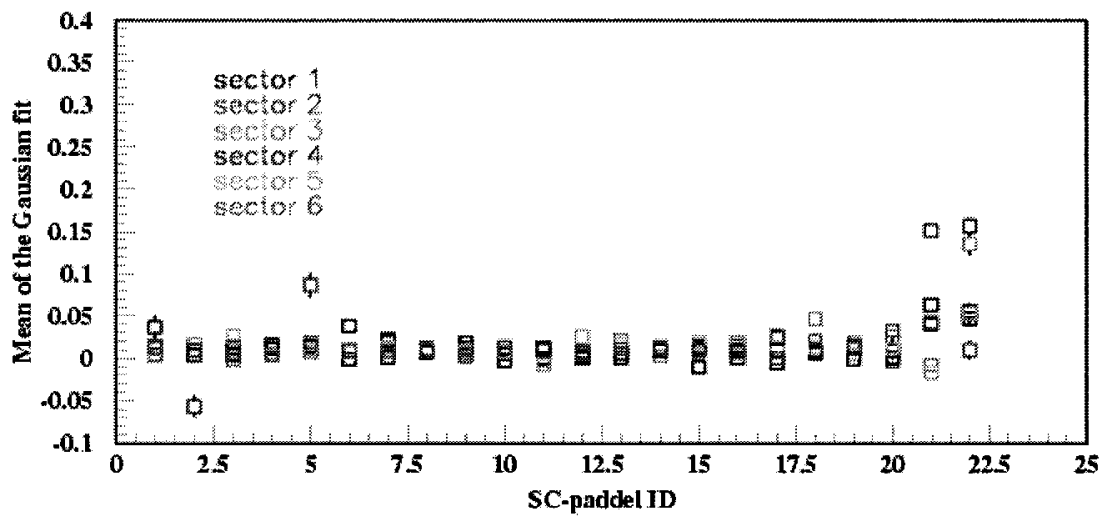

FIG. 47. The mean of the RF offset (in ns) as a function of the TOF paddle number for one EG1 run.

particles for a given event $i$ can then be calculated using,

$$
\beta=\frac{l_{i}}{c\left(T_{S C}^{i}-T_{\text {start }}^{e l}\right)} .
$$




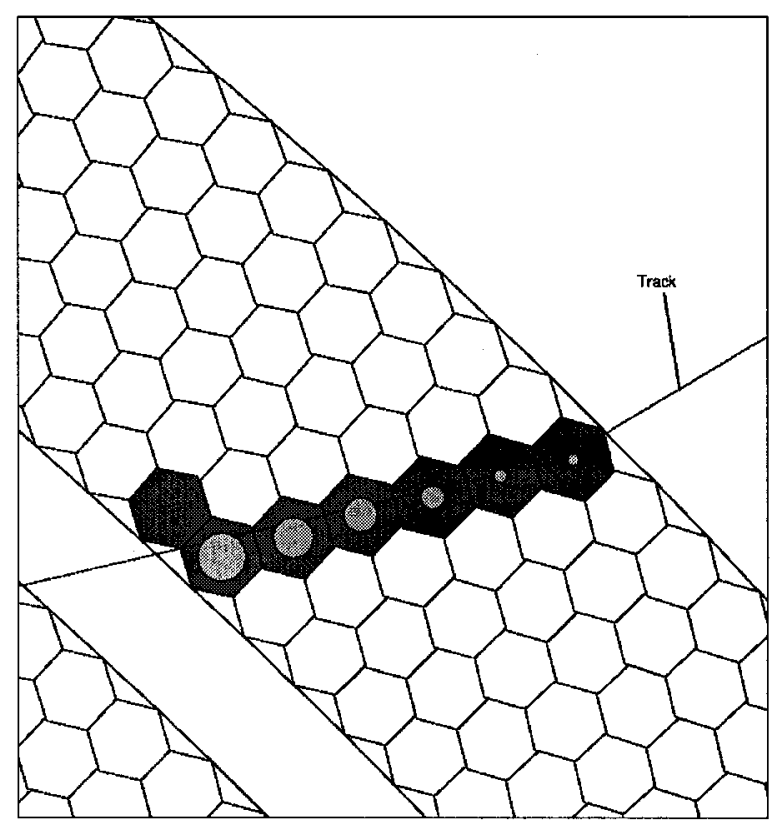

FIG. 48. A TBT track through a single superlayer. The circles are DOCA calculated using the drift velocity function. Details are in the text.

\subsection{TIME BASED TRACKING}

Once the start time is known, the recorded time of each DC cell is used in the final stage of track reconstruction. This method is called time based tracking (TBT). Although reconstruction software is available for constructing particle tracks using only hit based tracking, the data analyzed for this thesis only used tracks reconstructed with TBT. To do time based tracking the raw TDC time measured at each sense wire must be converted to a drift time, $t_{d r i f t}$, which is the time for electrons created by the passing charged particle to drift to the sense wire. The drift time is obtained as follows:

$$
t_{\text {drift }}=T_{\text {cable }}-T_{\text {start }}-t_{T D C}-t_{\text {flight }}-t_{\text {prop }}-t_{\text {walk }},
$$

where,

- $T_{\text {cable }}$ accounts for cable delays; the DC signal cables have different lengths.

- $T_{\text {start }}$ is the start time described in the previous section.

- $t_{T D C}$ is the raw time measured by the TDC, 
- $t_{\text {flight }}$ accounts for the flight time of the particle from the target to the sense wire and

- $t_{\text {walk }}$ is the time walk correction.

Charged particles with different momenta produce different amounts of electron ion pairs in the drift chamber gas. Since ionization is proportional to $1 / \beta^{2}$, high momentum particles ionize the gas less than low momentum particles. The larger the ionization the larger the signal and the sooner it crosses the discriminator threshold. Large momenta (low ionization) tracks are smeared to larger drift times. Therefore we have to subtract off a correction term from the time for high momentum tracks which is called the time walk correction. Since the signal size also depends on how far a track is from the sense wire of each cell the timewalk correction is a function of time.
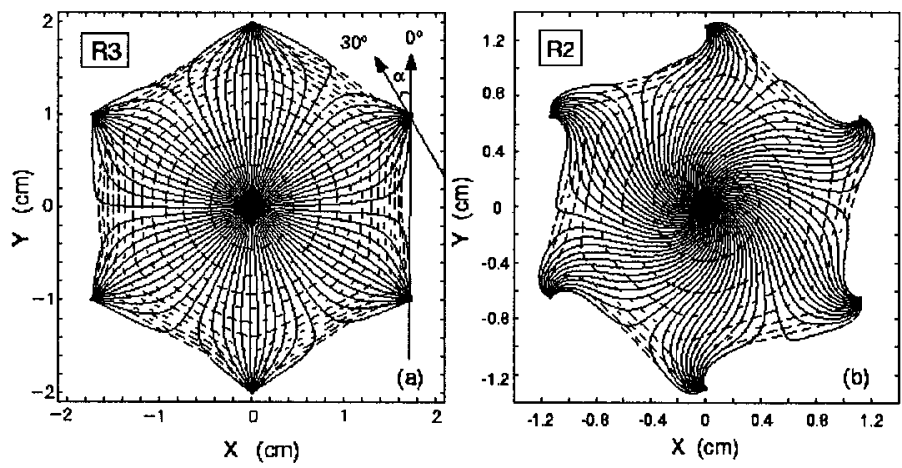

FIG. 49. The contours of constants time and electric field lines for a Region 3 (R3) and a Region 2 (R2) cell drawn using the GARFIELD program [42].

Once the drift time is calculated it is converted to the corresponding distance $\left(x_{\text {calcDOCA }}\right)$ which is the calculated DOCA (Distance Of Closest Approach), using the drift velocity function. The drift velocity function is the relation between the DOCA of a particle track and the drift time. The conversion of drift time to drift distance is complicated due to several factors,

- Since the drift cells and the resulting isochrones do not have the ideal circular shape (Fig. 49(a)), determining the drift distance requires a knowledge of the entry angle $(\alpha)$ of the track. 
- Since region 2 chambers are located in the torus magnetic field, the effective electric field lines of the cell (reflecting a combination of electric and magnetic force on the electron) rotate and shrink as shown in Fig. 49(b).

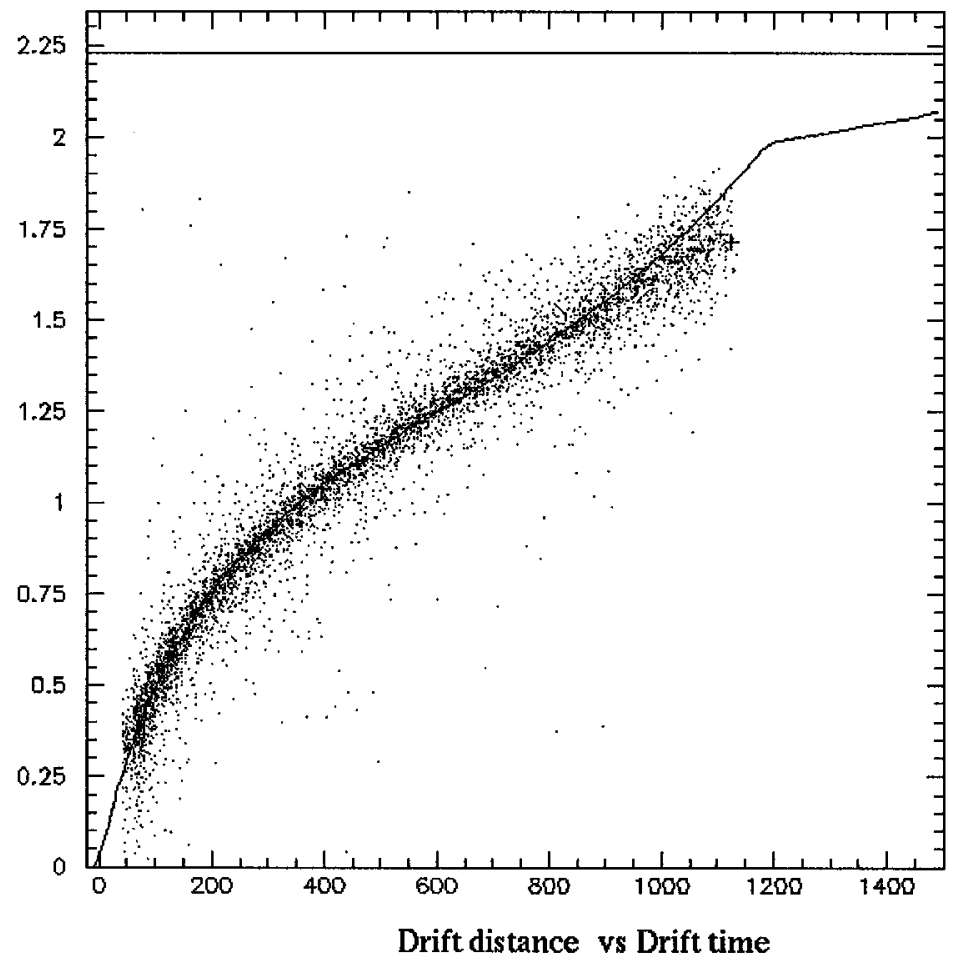

FIG. 50. The DOCA (cm) as a function of drift time (ns) for Sector 1 Super-layer 6 [51]. Also shown is the fitted drift velocity function described in the text.

The CLAS drift chambers are calibrated by parameterizing the drift velocity function for every superlayer in every sector. The functional form consists of a base function,

$$
x_{\text {calcDOCA }}(t)=v_{0} t+\eta\left(\frac{t}{t_{\text {max }}}\right)^{p}+\kappa\left(\frac{t}{t_{\text {max }}}\right)^{q},
$$

where $t=t_{d r i f t}, t_{\text {max }}$ is the time it takes for ions created in the drift cell to travel from the very edge of the drift cell to the sense wire and $v_{0}$ is the value of the saturated drift velocity near $t=0$. The parameters of the function, $p, q, \eta$ and $\kappa$, for each superlayer of each sector are obtained by doing a fit to the calculated DOCA as a function of drift time. An example is shown in Fig. 50. In addition to the base function, corrections for the non-circular cell shape and distortions caused by magnetic fields were properly included in the functional form.

After determining the calculated DOCA a circle with DOCA as the radius is defined around each sense wire that was hit. Then the tracking program does a least 
square fit to minimize the difference between the $x_{c a l c D O C A}$ and the distance of closest approach for each cell for the trial trajectory $x_{\text {fitDOCA }}$ (Fig. 48),

$$
\chi^{2}=\sum_{i} \frac{\left|x_{\text {calcDOCA }}-x_{f i t D O C A}\right|^{2}}{\sigma_{i}^{2}},
$$

where the uncertainty $\sigma_{i}$ is known for each drift chamber. The distance calculated from the track fit to each sense wire is called the fit DOCA.
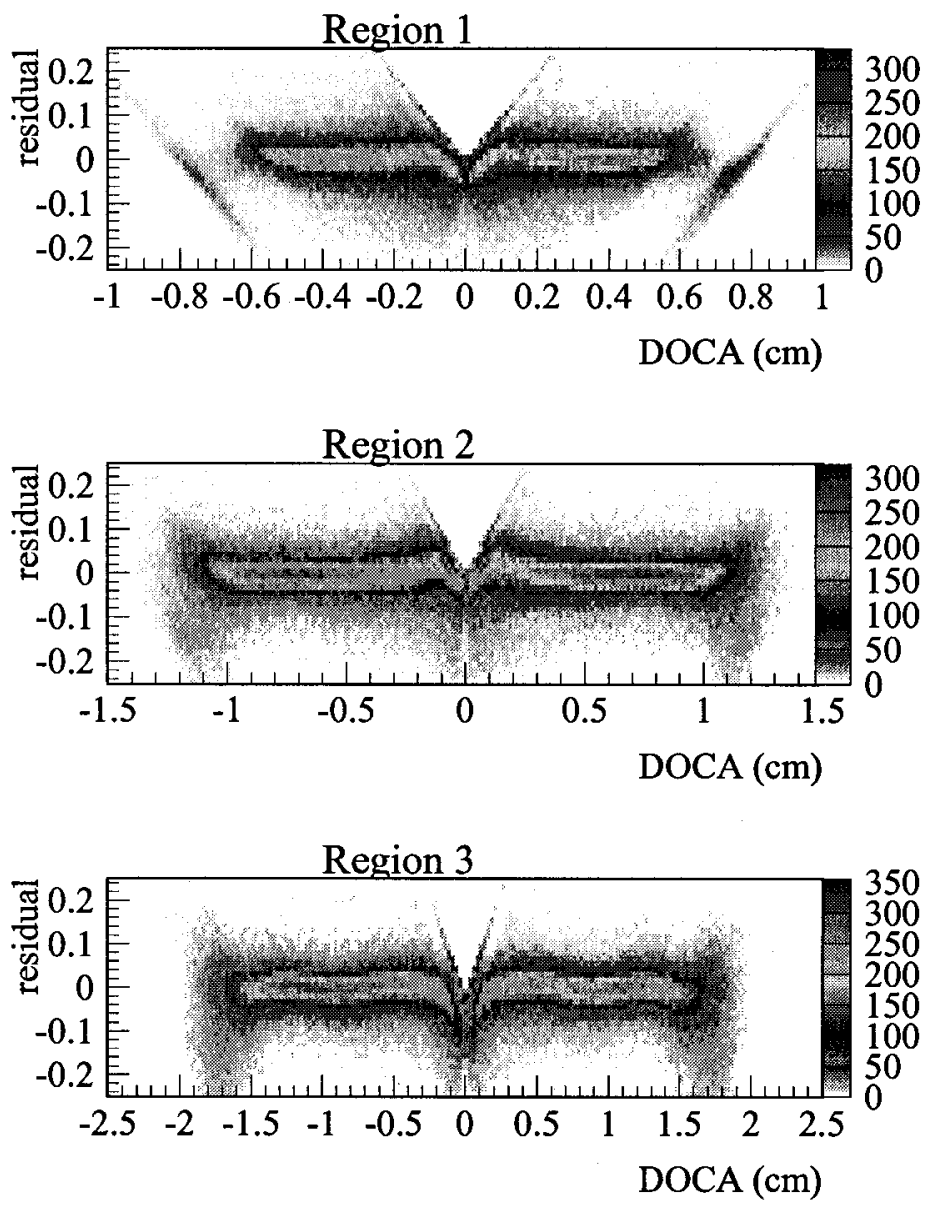

FIG. 51. DC residuals vs calculated DOCA for the three regions for sector 1 for one EG1 run. Data were taken at $5.6 \mathrm{GeV}$.

The difference between the absolute value of the calculated DOCA and the absolute value of the fit DOCA is defined as the residual for each cell and is the primary means of measuring the resolution of the drift chambers. When properly calibrated the residuals should not show any dependence on drift time or the calculated distance. An example of residual vs calculated DOCA is given in Fig. 51. 
In this analysis the calibration quality of each of the detectors were throughly checked before performing the data analysis. Fig. 52 shows the resolution of the drift chamber for $5.627 \mathrm{GeV}$ data. All the other detectors were also checked in a similar manner.

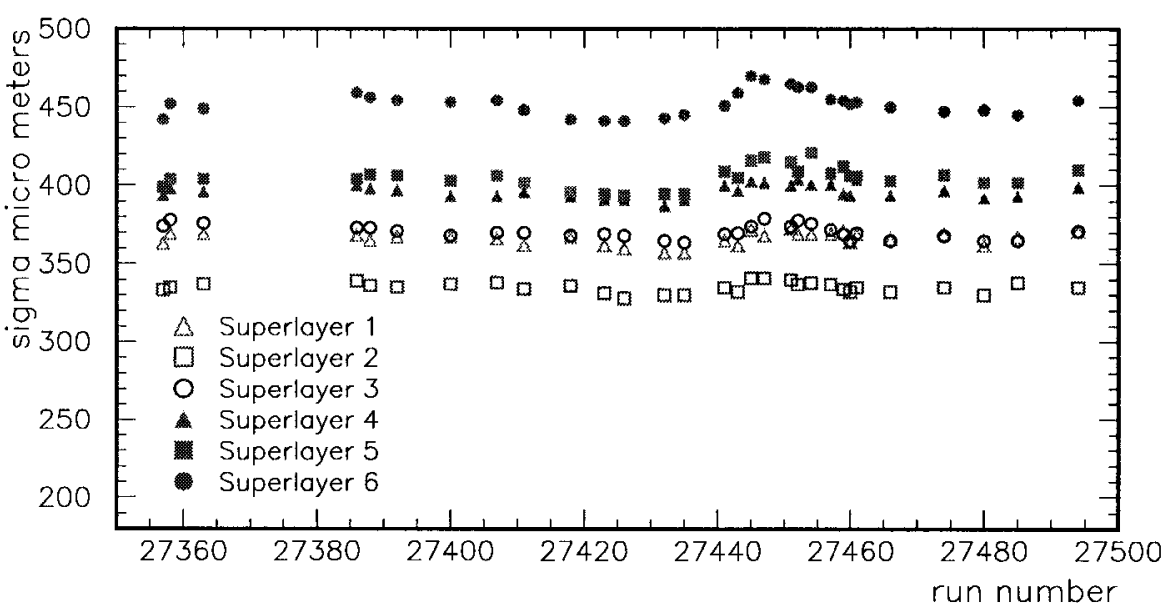

FIG. 52. DC resolution, which is the sigma of a double Gaussian fit to residuals, as a function of run number for the $5.6 \mathrm{GeV}$ inbending data.

Once the reconstruction of events are completed all the data files can be used for the physics analysis of the data. As explained above all the events are reconstructed using somewhat loose criteria. Before doing any physics analysis, each particle has to be properly identified using more careful requirements. The next chapter explains how this was performed and how the results were extracted. 


\section{CHAPTER 5}

\section{DATA ANALYSIS}

The first step in extracting $A_{1}, g_{1}$ and $\Gamma_{1}$ is to measure the asymmetry $A_{\|}$described in Chapter 1. The most important step in extracting these physics quantities is to identify the scattered electron and the helicity state properly. In addition, to extract $A_{\|}$, the target polarization and the background due to ${ }^{15} \mathrm{~N}^{\text {in }} \mathrm{ND}_{3}$ also have to be determined. In an inclusive analysis it is impossible to avoid the electron sample being contaminated by particles such as $\pi^{-}$and electrons originating from pair symmetric processes. Therefore, these effects have to be studied and corrected for. In this chapter the procedure used to extract the asymmetry $A_{\|}$from the data will be discussed.

\subsection{BEAM SETTINGS}

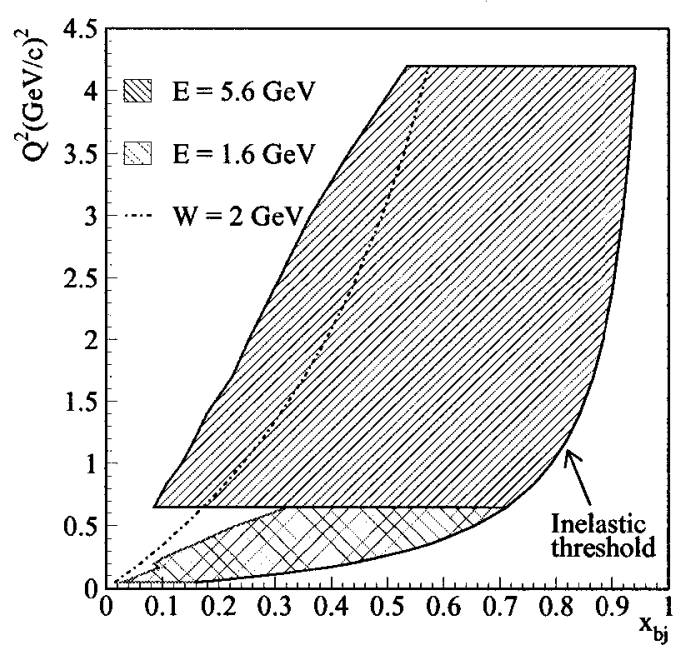

FIG. 53. The plot shows the kinematic coverage of the $5 . \mathrm{x} \mathrm{GeV}$ and $1 . \mathrm{x} \mathrm{GeV}$ data.

The entire EG1b experiment which ran from September 2000 to April 2001 includes four main data sets with four different beam energies, $1.6 \mathrm{GeV}, 2.6 \mathrm{GeV}, 4.2$ $\mathrm{GeV}$ and $5.7 \mathrm{GeV}$, each consisting of smaller data sets that have slightly different beam energies and different torus settings. This thesis presents the analysis and results of the $1.6 \mathrm{GeV}$ and $5.6 \mathrm{GeV}$ data (Table II). The kinematic coverage of these two beam settings are shown in Fig. 53. Each of the beam energy and torus 
TABLE II. Summary of data analyzed in this thesis.

\begin{tabular}{|l|c|c|r|}
\hline $\begin{array}{c}\text { Name used } \\
\text { in the text }\end{array}$ & $\begin{array}{c}\text { beam energy } \\
(\mathrm{GeV})\end{array}$ & Targets & Torus setting \\
\hline $1 . \mathrm{x} \mathrm{GeV}$ & 1.606 & $\mathrm{ND}_{3}, \mathrm{NH}_{3},{ }^{12} \mathrm{C},{ }^{4} \mathrm{He}$ & Inbending \\
& 1.606 & $\mathrm{ND}_{3}, \mathrm{NH}_{3},{ }^{2} \mathrm{C},{ }^{4} \mathrm{He}$ & Outbending \\
& 1.724 & $\mathrm{ND}_{3}, \mathrm{NH}_{3},{ }^{2} \mathrm{C},{ }^{4} \mathrm{He}$ & Outbending \\
& 1.724 & ${ }^{15} \mathrm{~N}$ & Inbending \\
& 1.724 & ${ }^{15} \mathrm{~N},{ }^{12} \mathrm{C}$ & Outbending \\
\hline $5 . \mathrm{x} \mathrm{GeV}$ & 5.764 & $\mathrm{ND}_{3}, \mathrm{NH}_{3},{ }^{12} \mathrm{C},{ }^{4} \mathrm{He}$ & Outbending \\
& 5.735 & $\mathrm{ND}_{3}, \mathrm{NH}_{3},{ }^{12} \mathrm{C},{ }^{4} \mathrm{He}$ & Outbending \\
& 5.735 & $\mathrm{ND}_{3}, \mathrm{NH}_{3},{ }^{12} \mathrm{C},{ }^{4} \mathrm{He}$ & Inbending \\
& 5.627 & $\mathrm{ND}_{3}, \mathrm{NH}_{3},{ }^{2} \mathrm{C},{ }^{4} \mathrm{He}$ & Inbending \\
& 5.627 & ${ }^{15} \mathrm{~N},{ }^{12} \mathrm{C}$ & Inbending \\
& 5.627 & ${ }^{15} \mathrm{~N},{ }^{12} \mathrm{C}$ & Outbending \\
\hline
\end{tabular}

configurations listed in Table II were analyzed separately and combined at the end.

\subsection{RUN SELECTION}

To select runs to be used in the analysis quality checks on a run by run basis were performed. This prevents us from using data that may have been taken during detector break downs and using files which may have been corrupted due to problems in the data acquisition system. Detector performance was checked by looking at the inclusive electron rates for each sector separately. Fig. 54 shows the rates for each sector for the $5.6 \mathrm{GeV}$ data. Ideally, the rates should be constant with run number. Runs that showed large deviations from the other runs were removed from the data set.

In a double polarization asymmetry analysis it is important to determine the sign of the product of beam and target polarization correctly. Combining runs which have different signs for the product would dilute the asymmetries and hence produce wrong results. The sign of the product of beam and target polarization for each run was studied by integrating the elastic asymmetry in the $0.88(\mathrm{GeV}) \leq W \leq 1.0(\mathrm{GeV})$ region and checking that it was positive. However, because of poor statistics in the elastic region this method could not be applied for the 5.x GeV data. For all 5.x 

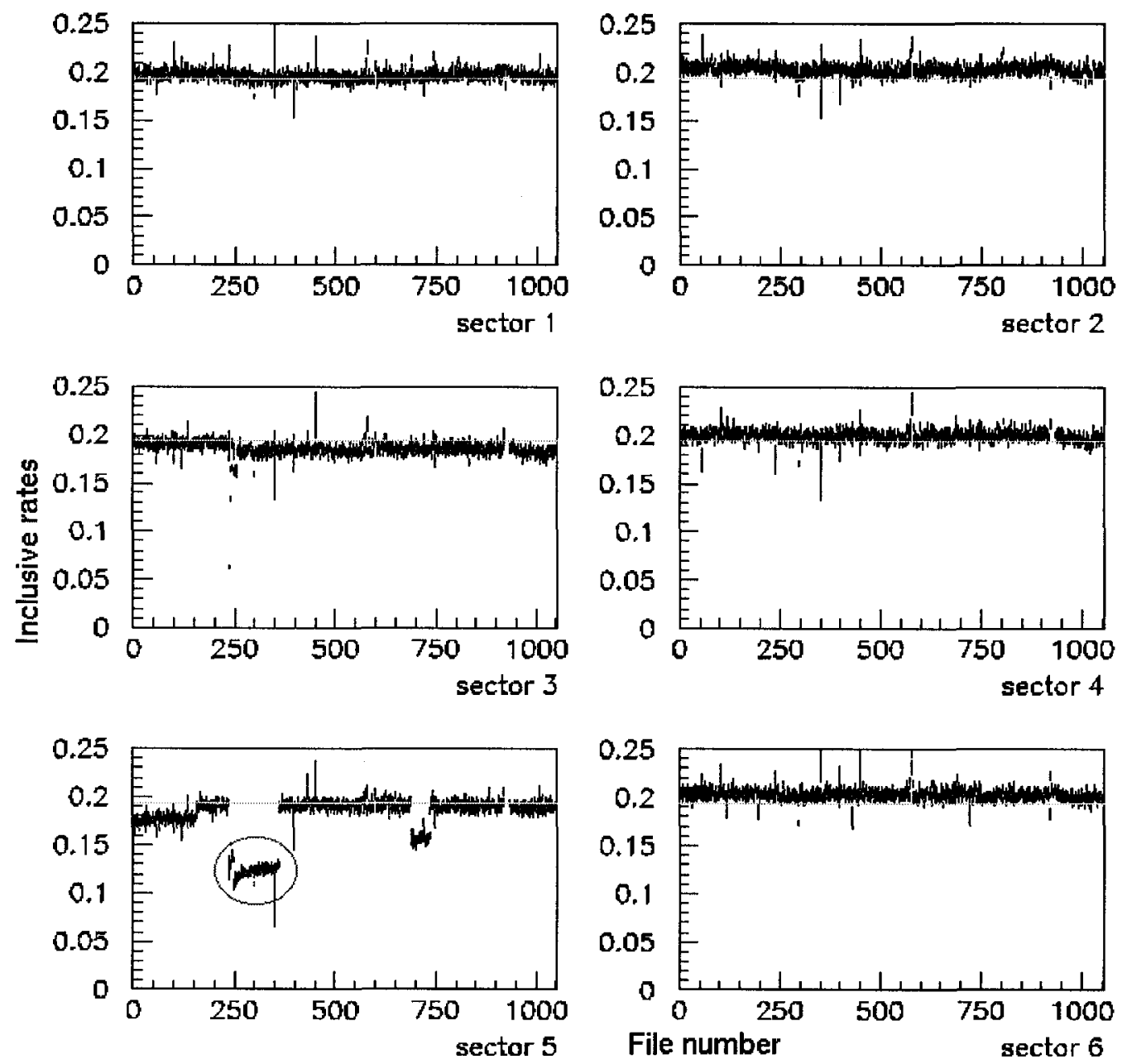

FIG. 54. The inclusive electron rates for each sector as a function of the file number [52]. The set of files circled are an example of bad files. Those files were not included in the analysis. 


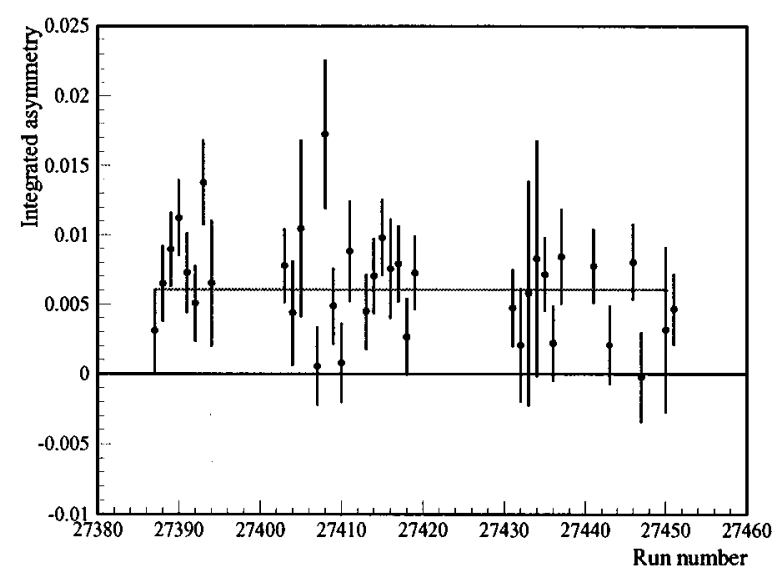

FIG. 55. Integrated asymmetry as a function of run number for the $5.627 \mathrm{GeV}$ data. The asymmetry has been integrated between $1.7(\mathrm{GeV}) \leq W \leq 2.5(\mathrm{GeV})$.

$\mathrm{GeV}$ data, the asymmetry in the range $1.7(\mathrm{GeV}) \leq W \leq 2.5(\mathrm{GeV})$ was integrated to check the sign of the product of beam and target polarization. The integrated inelastic asymmetry for the $5.627 \mathrm{GeV}$ data are shown in Fig. 55, which shows the same sign for all runs.

In addition the number of events scattered as a function of the beam position on the target was studied (Fig. 56). Runs where the beam was mis-steered and hit the target cup were removed from the runs selected to be used in the analysis.

\subsection{BIN SELECTION}

Since this analysis is part of a larger analysis project it was agreed to use a common set of $W$ and $Q^{2}$ bins. A $W$ bin width of $10 \mathrm{MeV}$ and the $Q^{2}$ bins listed in Table III were chosen. The primary reason for choosing small $W$ bins was to enable us to do all the integrals with a smaller step size, which makes the approximation integral $=$ sum a fair assumption. However, depending on statistics the results for $A_{1}$ and $g_{1}$ are plotted and tabulated in larger $W$ bins.

\subsection{FIDUCIAL CUTS}

The purpose of applying fiducial cuts is to remove inefficient regions of detectors where the acceptance is poorly understood. The advantage of doing an asymmetry analysis is that the detector efficiencies cancel out in the asymmetry given in equation 
TABLE III. The table lists the $Q^{2}$ bins used in the analysis. $Q_{n o m}^{2}$ is the number used in tables and labels. $Q_{\min }^{2}$ and $Q_{\max }^{2}$ are the upper and the lower limit of the bin. $\left\langle Q^{2}\right\rangle$ is the actual arithmetic mean of the bin limit.

\begin{tabular}{|c|c|c|c|}
\hline$Q_{\text {nom }}^{2}\left(\mathrm{GeV}^{2}\right)$ & $Q_{\min }^{2}\left(\mathrm{GeV}^{2}\right)$ & $Q_{\max }^{2}\left(\mathrm{GeV}^{2}\right)$ & $\left\langle Q^{2}\right\rangle\left(\mathrm{GeV}^{2}\right)$ \\
\hline 0.05 & 0.0452 & 0.054 & 0.0496 \\
0.06 & 0.0540 & 0.0645 & 0.0592 \\
0.07 & 0.0645 & 0.0770 & 0.0707 \\
0.084 & 0.0770 & 0.0919 & 0.0844 \\
0.1 & 0.092 & 0.110 & 0.101 \\
0.12 & 0.110 & 0.131 & 0.120 \\
0.14 & 0.131 & 0.156 & 0.144 \\
0.17 & 0.156 & 0.187 & 0.171 \\
0.2 & 0.187 & 0.223 & 0.205 \\
0.24 & 0.223 & 0.266 & 0.244 \\
0.3 & 0.266 & 0.317 & 0.292 \\
0.35 & 0.317 & 0.379 & 0.348 \\
0.42 & 0.379 & 0.452 & 0.416 \\
0.5 & 0.452 & 0.540 & 0.496 \\
0.6 & 0.540 & 0.645 & 0.592 \\
0.7 & 0.645 & 0.770 & 0.707 \\
0.84 & 0.770 & 0.919 & 0.844 \\
1.0 & 0.92 & 1.10 & 1.01 \\
1.2 & 1.10 & 1.31 & 1.20 \\
1.4 & 1.31 & 1.56 & 1.44 \\
1.7 & 1.56 & 1.87 & 1.71 \\
2.0 & 1.87 & 2.23 & 2.05 \\
2.4 & 2.23 & 2.66 & 2.44 \\
3.0 & 2.66 & 3.17 & 2.92 \\
3.5 & 3.17 & 3.79 & 3.48 \\
4.2 & 3.79 & 4.52 & 4.16 \\
\hline
\end{tabular}




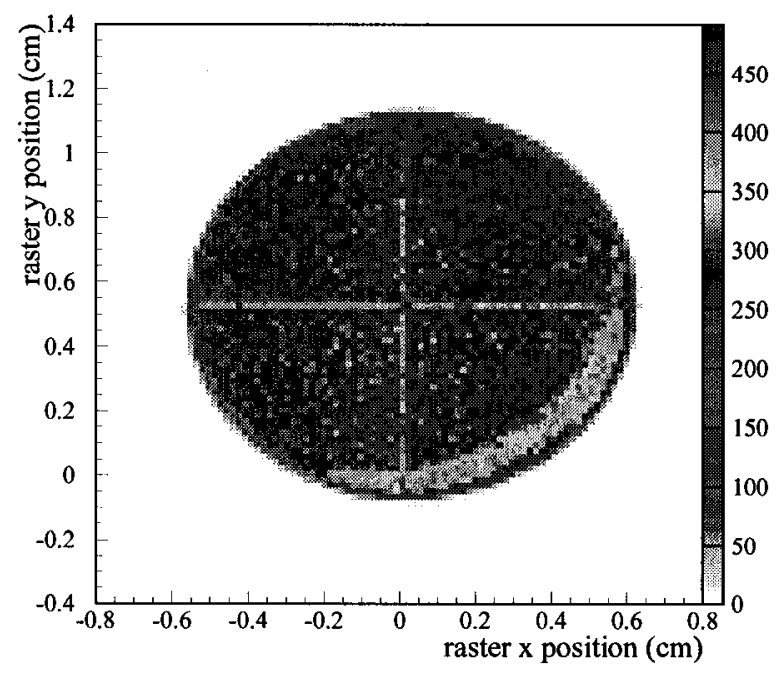

FIG. 56. The plot shows the number of events scattered as a function of raster $x$ position and raster $y$ position. The concentration of events on the bottom right indicates that the beam was hitting the target cup. Because of that, this run was not included in the analysis.

16. However, the carbon and helium runs that are used to remove the nitrogen background from $\mathrm{ND}_{3}$ have to have the same acceptance as the $\mathrm{ND}_{3}$ runs. Fluctuation of acceptance between these different target types was minimized by taking carbon and He runs in the middle of the $\mathrm{ND}_{3}$ runs. However, due to rapid fluctuation of the Cerenkov efficiency at the edges of the detector (Fig. 57) it was necessary to apply fiducial cuts to ensure the same acceptance for the $\mathrm{ND}_{3}$, carbon and helium runs. Therefore fiducial cuts were determined by evaluating the Cerenkov efficiency in the $\theta, \phi$ plane. For each bin in $\theta$ and $\phi$ the number of Cerenkov photoelectrons is assumed to be in a Poisson distribution. Therefore the inefficiency for a given bin can be written in terms of the expected average number of photo-electrons $(\mu)$ and the minimum detection threshold (or cuts) in photo-electrons $(c)$ as,

$$
\text { Inefficiency }=\sum_{n=0}^{n<c} \frac{\mu^{n} e^{-\mu}}{n !} .
$$

To determine our fiducial cuts first a value for $\mu$ was calculated from equation (131) by using a predetermined efficiency and photoelectron cut. For our analysis an efficiency of $80 \%$ was determined to be reasonable. For each data set $\mu$ was calculated according to the photoelectron cut that was employed for that particular data set.

The fiducial cuts were further complicated by the additional magnetic field present 


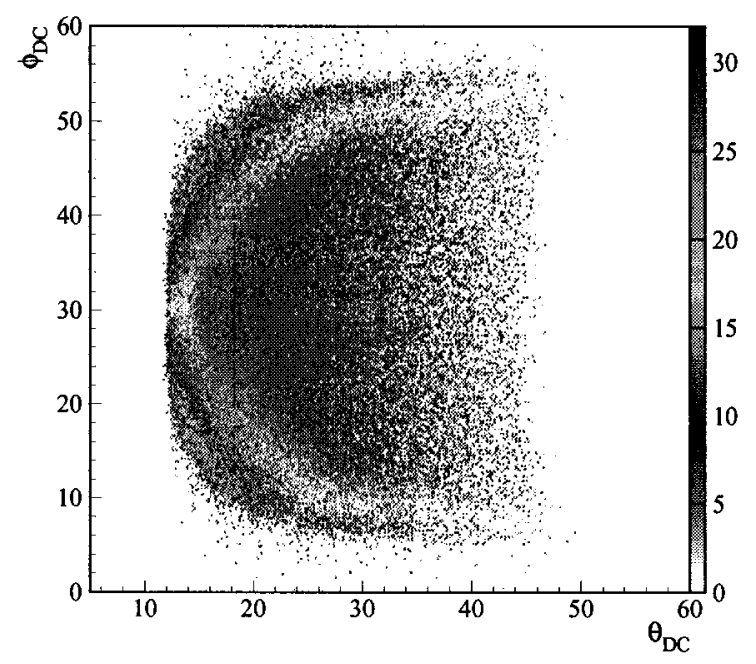

FIG. 57. The number of photoelectrons vs $\phi_{D C}$ vs $\theta_{D C}$ for momentum between 1 and $3 \mathrm{GeV}$. The inefficient regions at the edges, where no photoelectrons are recorded, are clearly visible.

due to the polarized target. This axial field causes the charged particles leaving the target to experience a change in $\phi$. Therefore the $\phi$ angle reconstructed by the drift chambers is shifted with respect to the vertex $\phi$ as shown in Fig. 58. The fiducial cuts were applied to $\theta$ and $\phi$ at the drift chamber layer $1\left(\theta_{D C}, \phi_{D C}\right)$ where the acceptance is symmetrical.

For inbending electrons this inefficiency has been studied and documented [53]. The Cerenkov efficiency function was used to plot the efficiency as a function of $\theta$ and $\phi$. The fiducial cuts were chosen so that the accepted region had an efficiency greater than $80 \%$. However for outbending electrons the Cerenkov efficiency is not known. Therefore, to determine the fiducial cut, the average number of photoelectrons in the $\theta_{D C}, \phi_{D C}$ plane were plotted using the data and the inefficient regions of the Cerenkov counter were identified.

For inbending electrons the fiducial cut was defined as follows:

$$
30-\Delta \phi<\phi_{s e c}<30+\Delta \phi \text { and } \theta_{c u t}<\theta_{D C}<45
$$

where $\phi_{\text {sec }}$ is the $\phi$ angle at the DC layer 1 in sector coordinates and

$$
\begin{gathered}
\Delta \phi=A\left(\sin \left(\theta_{D C}-\theta_{\text {cut }}\right)\right)^{\text {expon }}, \\
\text { expon }=B\left(P_{\text {el }} \frac{3375}{I_{\text {torus }}}\right)^{C},
\end{gathered}
$$



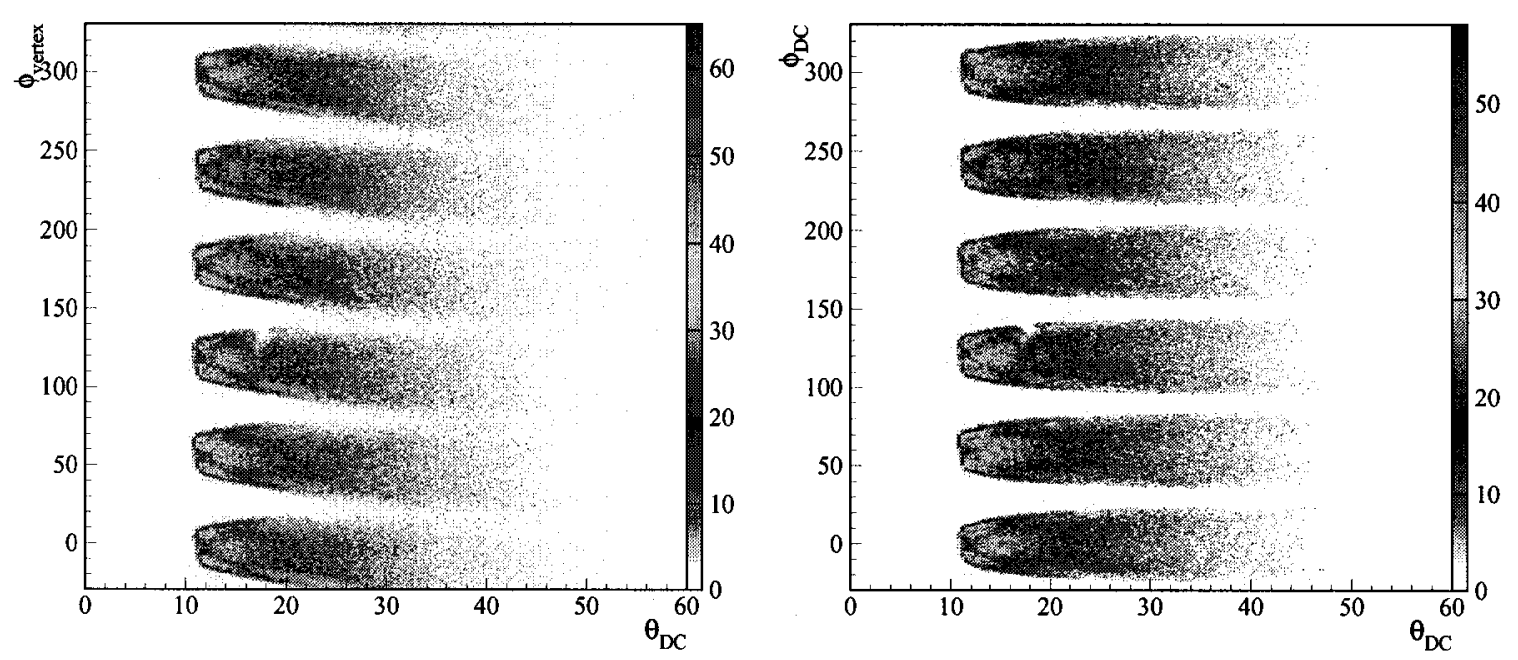

FIG. 58. Comparison of the $\phi$ angle at the vertex (left) and the $\phi$ angle at the DC layer 1 (right) as a function of $\theta$. The distortion in the distribution in the vertex coordinates is due to the target magnetic field.

$$
\text { and } \quad \theta_{c u t}=D+\frac{E}{\left(P_{e l}+F\right) \frac{3375}{I_{\text {torus }}}},
$$

where $P_{e l}$ is the momentum of the electron and $I_{\text {torus }}$ is the torus current. $\theta_{c u t}$ is the minimum accepted theta, which depends on momentum and is always smaller than the parameter $D$. The parameter $E$ takes into account how much a particle's trajectory is bent due to the torus magnetic field, the higher the momentum the smaller the cut should be. Parameter $F$ controls how rapidly the function should change with momentum. Parameters $A, B$, and $C$ control the "tightness" of the cut in $\phi$. The parameters $A$ through $F$ for each data set were determined empirically. Further for $P_{e l}<3 \mathrm{GeV}$ and $12<\phi_{D C}<48, \Delta \phi=18$ was used. Since a different requirement for the number of photoelectrons was used for $P_{e l}>3 \mathrm{GeV}$, a different set of constants for parameters $A$ through $F$ were chosen, corresponding to a much "looser" cut than the $P_{e l}<3 \mathrm{GeV}$ case. Fig. 59 shows the applied fiducial cuts for some of the momentum bins.

For outbending electrons the center of the detector consisted of prominent inefficient regions (Fig. 60). Therefore, in addition to the edges, the center also had to be removed. For outbending electrons the following fiducial cut was applied;

$$
30-\Delta \phi<\phi_{s e c}<30+\Delta \phi \text { and } \theta_{c u t}<\theta_{D C}<\theta_{\max }
$$



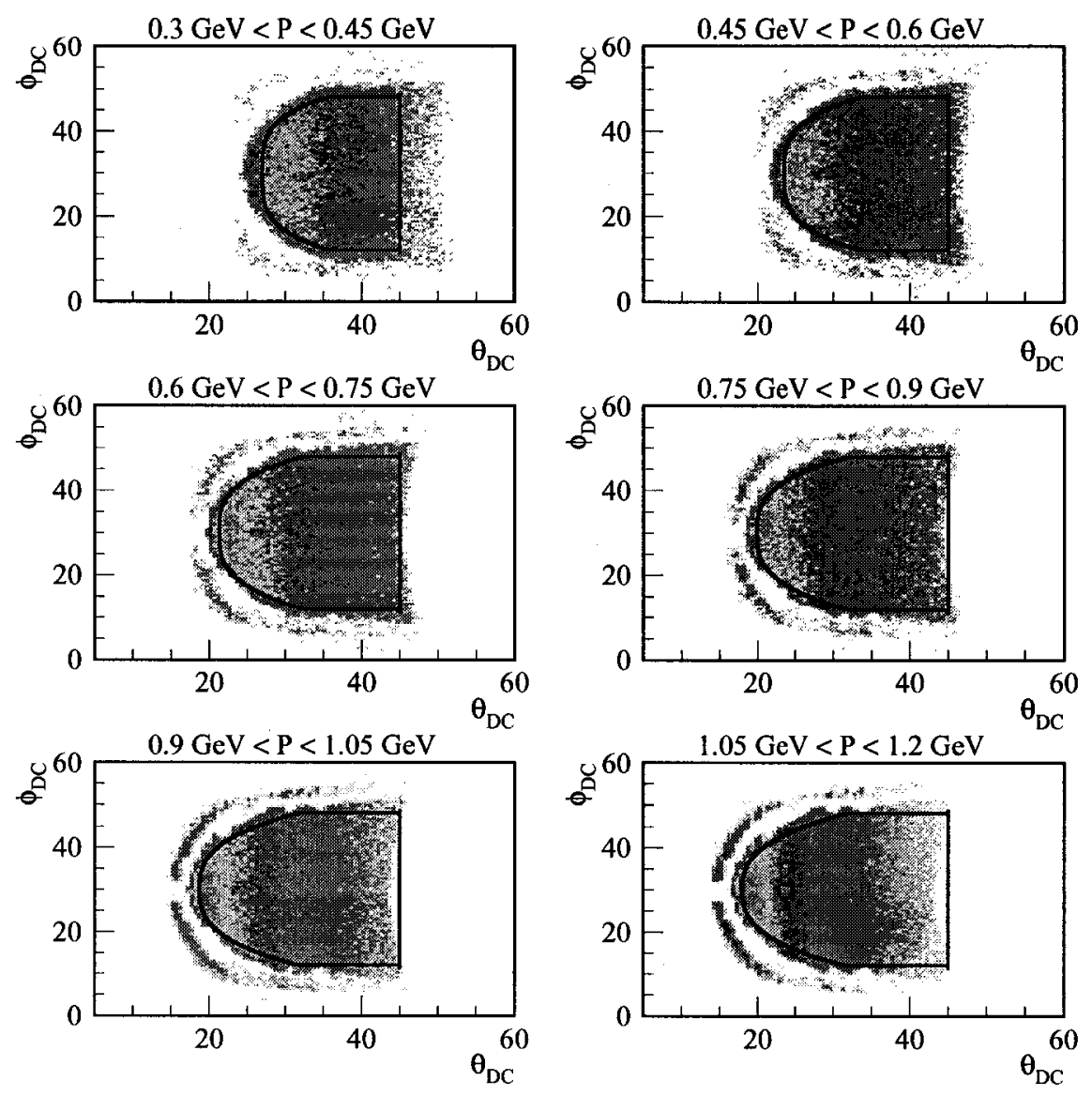

FIG. 59. The number of events scattered from the regions where the Cerenkov efficiency is greater than $80 \%$. The black line outlines the boundary of the applied fiducial cut. Events inside the lines were accepted. These are inbending $5.6 \mathrm{GeV}$ data.

where

$$
\begin{gathered}
\Delta \phi=A^{\prime}\left(\sin \left(\theta_{D C}-6.5\right)\right)^{\text {expon }} \\
\text { expon }=B^{\prime}\left(P_{\text {scale }} / 4\right)^{C^{\prime}} \\
\theta_{\text {cut }}=D^{\prime}+E^{\prime}\left(1-P_{\text {scale }} / 4\right)^{F^{\prime}} \\
\theta_{\max }=\min \left(40, \theta_{\text {nom }}\right) \\
\left.\theta_{\text {nom }}=35\left[\left(P_{\text {el }} \frac{3375}{\left|I_{\text {torus }}\right|}+2.5\right)\right) \frac{1}{5}\right]^{1 / 3} \text { and } P_{\text {scale }}=P_{\text {el }} \frac{1500}{\left|I_{\text {torus }}\right|} .
\end{gathered}
$$

To remove the center the following cut was used,

$$
30+\phi_{\text {center }}<\phi_{\text {sec }}<30-\phi_{\text {center }}
$$




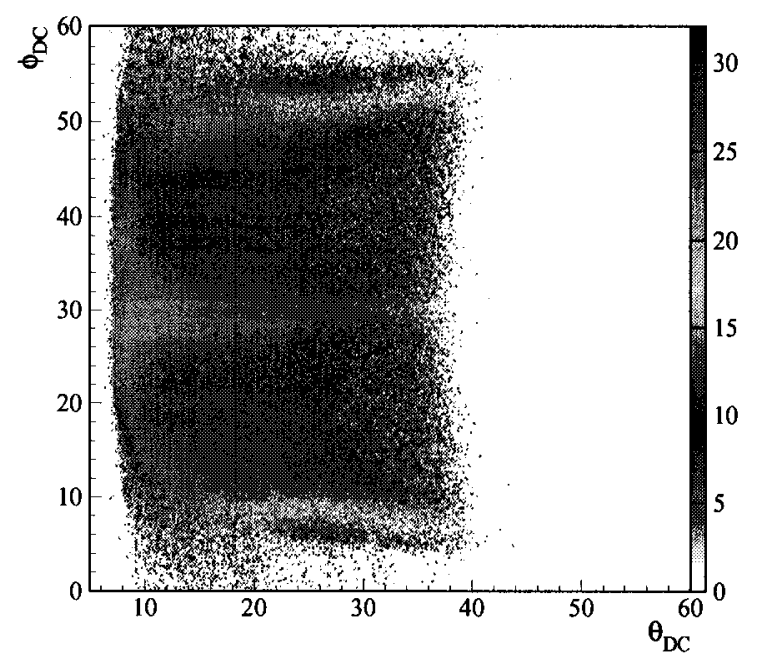

FIG. 60. Same as Fig. 57 but for outbending torus current. Notice the large inefficiency in the center of the detector in addition to the inefficiencies observed at the edges.

where,

$$
\phi_{\text {center }}=\frac{G^{\prime}}{\sin \left(\theta_{D C}+H^{\prime}\right)} .
$$

Similarly parameters $A^{\prime}$ through $H^{\prime}$ were chosen empirically. Depending on the inefficiencies in the center, sector dependent $\phi_{\text {center }}$ cuts were used. Fig. 61 shows the fiducial cuts for few momentum bins for the outbending torus settings.

\subsection{ELECTRON IDENTIFICATION}

The hardware trigger used during data taking required signals in the electromagnetic calorimeter and the Cerenkov counter in coincidence. The offline track reconstruction code uses a more restricting definition of a possible electron candidate and rejects some of the events which passed the trigger requirements. However, for the physics analysis, more careful cuts have to be used in order to minimize contamination by other negatively charged particles. Therefore in this analysis electrons were accepted only if the following criteria were satisfied:

1. A good track identified by the track reconstruction code which is determined using time based tracking.

2. A hit in Cerenkov counter and the EM calorimeter which corresponds to 

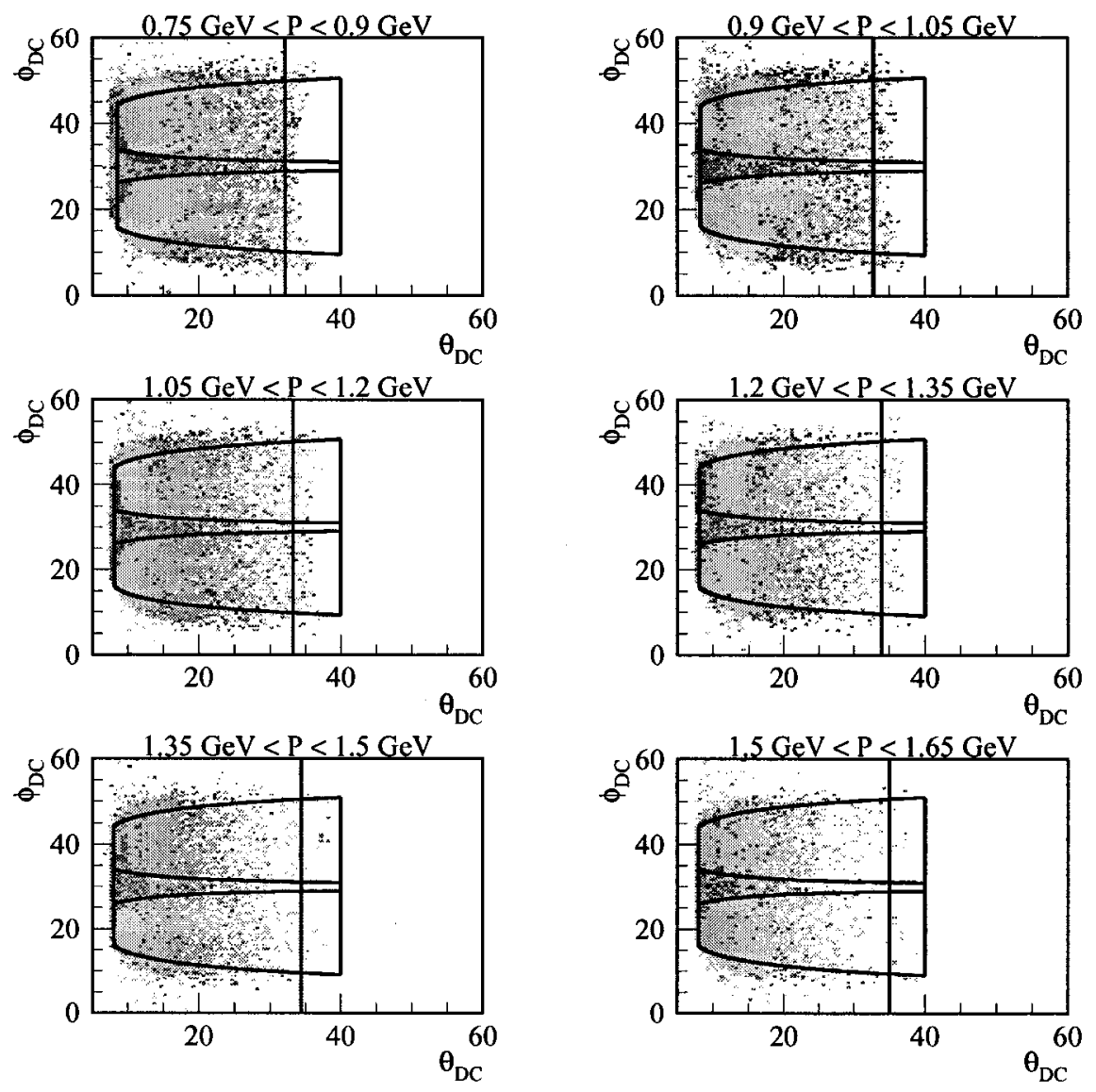

FIG. 61. Fiducial cuts for outbending torus settings. The gray shaded area represents the region where the Cerenkov efficiency is greater than $80 \%$. The black line outlines the boundary of the applied cut. The center was removed using a cut similar to the one shown in the figure.

trigger bits 1 through 6 .

3. The momentum of the electron satisfies $0.15 E_{\text {beam }}<P_{e l}<E_{\text {beam }}$ for $5 . \mathrm{x} \mathrm{GeV}$ data and $0.5 \mathrm{GeV}<P_{e l}<E_{\text {beam }}$ for $1 . \mathrm{x} \mathrm{GeV}$ data, where $E_{\text {beam }}$ is the beam energy. In addition to rejecting pions the low momentum cut also rejects events with high radiative effects.

4. It is within the fiducial region.

5. Charge $=-1$.

6. It satisfies the Cerenkov photoelectron requirement (see section 5.5.1). 
7. It passes cuts on the energy deposited in the EM calorimeter (see section 5.5.2).

8. $\mathrm{Z}$ vertex cut (see section 5.5.3).

The following subsections will describe the cuts 6,7 and 8 in detail.

\subsubsection{Cerenkov Cut}

The Cerenkov counter provides a separation of electrons from pions. This is possible because pions have a much higher threshold $(2.7 \mathrm{GeV})$ than electrons (9 $\mathrm{MeV})$. However separation of pions from electrons for pions with momentums above $2.7 \mathrm{GeV}$ becomes difficult because above the threshold velocity of the pion is large enough to produce Cerenkov light.

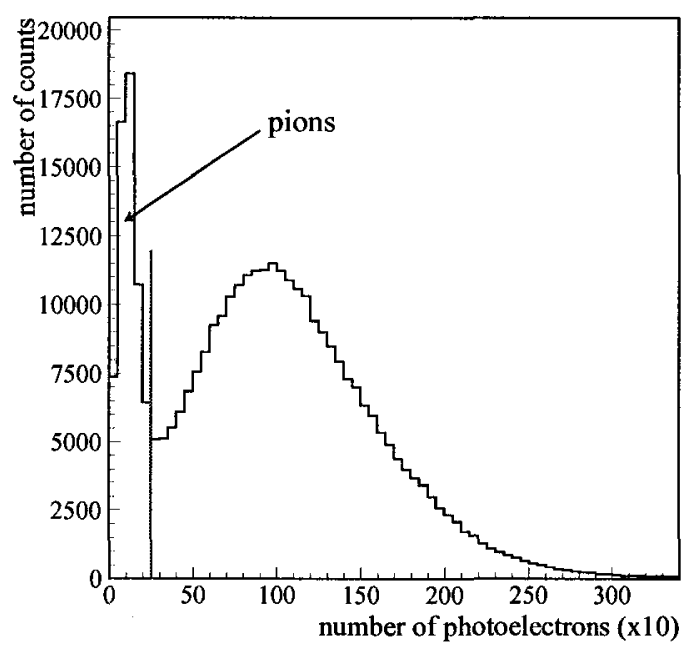

FIG. 62. The number of photoelectrons detected in the Cerenkov counter for 5.625 $\mathrm{GeV}$ momentum $<3 \mathrm{GeV}$ data. The red line indicates the applied cut.

Since most of the Cerenkov inefficiencies were removed using fiducial cuts, all the particles that gave a small signal in the Cerenkov counter were assumed to be pions which were misidentified as electrons. Depending on the beam setting and the momentum, different Cerenkov cuts were used to remove these pions,

- For the 5.x GeV data $\left(\mathrm{P}_{\mathrm{el}}<3 \mathrm{GeV}\right)$ events with a photoelectron signal $>2.5$ were accepted,

- For the 5.x GeV data $\left(\mathrm{P}_{\mathrm{el}}>3 \mathrm{GeV}\right)$ events with a photoelectron signal $>0.5$ were accepted, 
- For the 1.x GeV data events with a photoelectron signal > 2 were accepted.
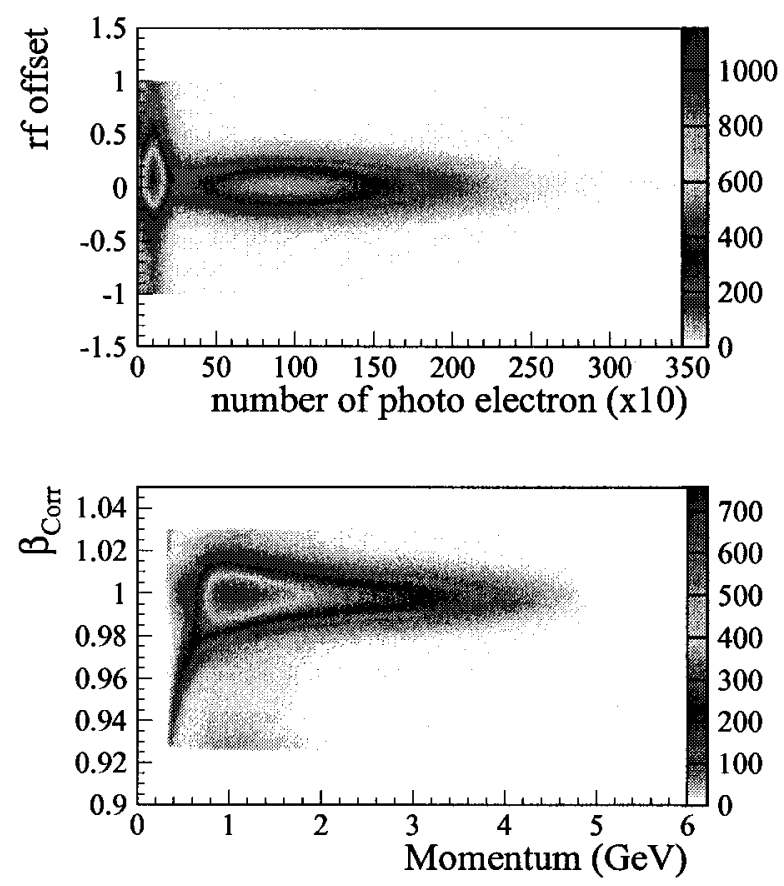

FIG. 63. The upper plot shows the rf offset vs the number of photoelectrons $(\times 10)$. The concentration of events below 2.5 photoelectrons are not centered around zero and have wrong start times. The lower plot is the reconstructed beta for the particles shown in the upper plot after correcting for the additional distance traveled by the particles.

To ensure that the particles which have a photoelectron signal smaller than the photoelectron cut are not electrons, the time of flight mass of these particles was reconstructed using the $\mathrm{rf}$ offset. Since we do not have enough information to independently calculate the beta of these misidentified particles, which is required to

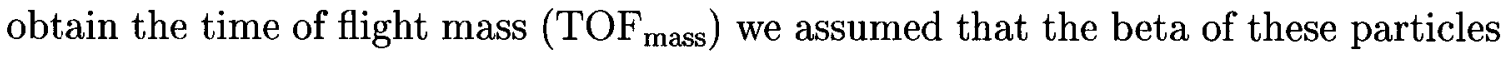
is close to one, but then made a small correction due to the measured additional travel time which is encoded in rf offset (Fig. 63),

$$
\begin{array}{ll}
\beta<1.3 & \beta_{\text {Corr }} c=\left(\frac{\text { tof path length }}{\text { tof path length } / c+\mathrm{rf} \mathrm{offset}}\right) \\
\beta>1.3 & \beta_{\text {Corr }} c=\left(\frac{\text { tof path length }}{\text { tof path length } / c+\mathrm{rf} \text { offset }+2.004}\right),
\end{array}
$$

where $c$ is the speed of light. The reconstructed TOF mass with and without photoelectron cuts for the $5 . \mathrm{x} \mathrm{GeV}$ inbending data is plotted in Fig. 64. Events for which 
the number of photoelectrons was less than the photoelectron cut are peaked around a mass close to the pion mass, confirming the argument. For the 1.x GeV data, unlike the 5.x GeV data, the percentage of misidentified electrons was very small.

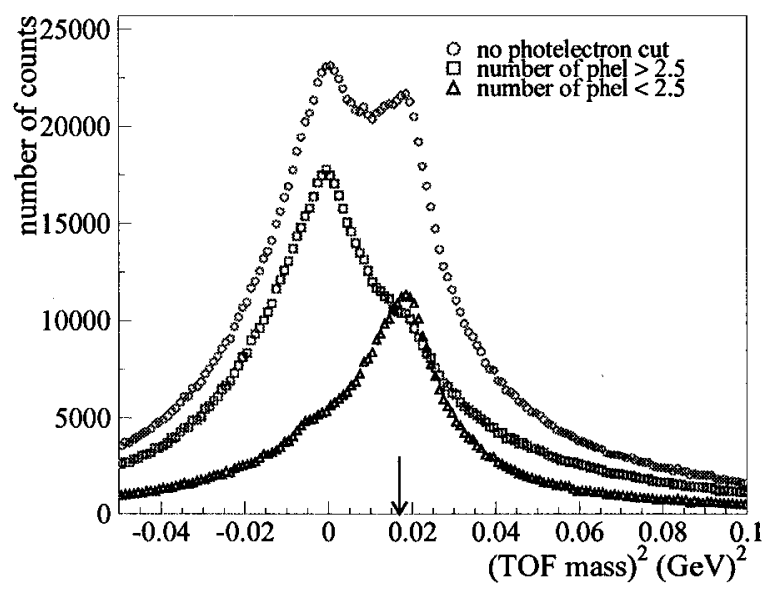

FIG. 64. The reconstructed TOF mass after applying different photoelectron cuts. The TOF mass for events with number of photoelectrons (phel) less than 2.5 is peaked around the pion mass which is indicated by an arrow in the plot.

\subsubsection{Electromagnetic Calorimeter Cuts}

The Cerenkov counter provides a good separation between electrons and pions particularly for momenta less than $2.7 \mathrm{GeV}$. Additional cuts based on the manner in which particles deposit their energy in the EM calorimeter can be used to further separate pions from electrons. The primary energy loss mechanism for electrons in the calorimeter is through pair production and subsequent showering reactions, while pions lose most of their energy through ionization. The total energy deposited by an electron in the EM calorimeter, $E_{t o t}$, is proportional to its momentum,

$$
\frac{E_{t o t}}{P_{e l}}=\alpha_{s}
$$

The constant of proportionality, known as the sampling fraction, $\alpha_{s}$, is a characteristic of the material used. Pions, on the other hand, are minimum ionizing and deposit a small amount of energy in the calorimeter; the energy they deposit is independent of the pion energy and depends only on the material and the detector thickness. Electrons lose most of their total energy in the inner part of the calorimeter while pions lose only a small fraction of their energy in the inner part of the calorimeter.Because the ratio of the inner to outer calorimeter thickness is $5 / 8$ the energy deposited by 

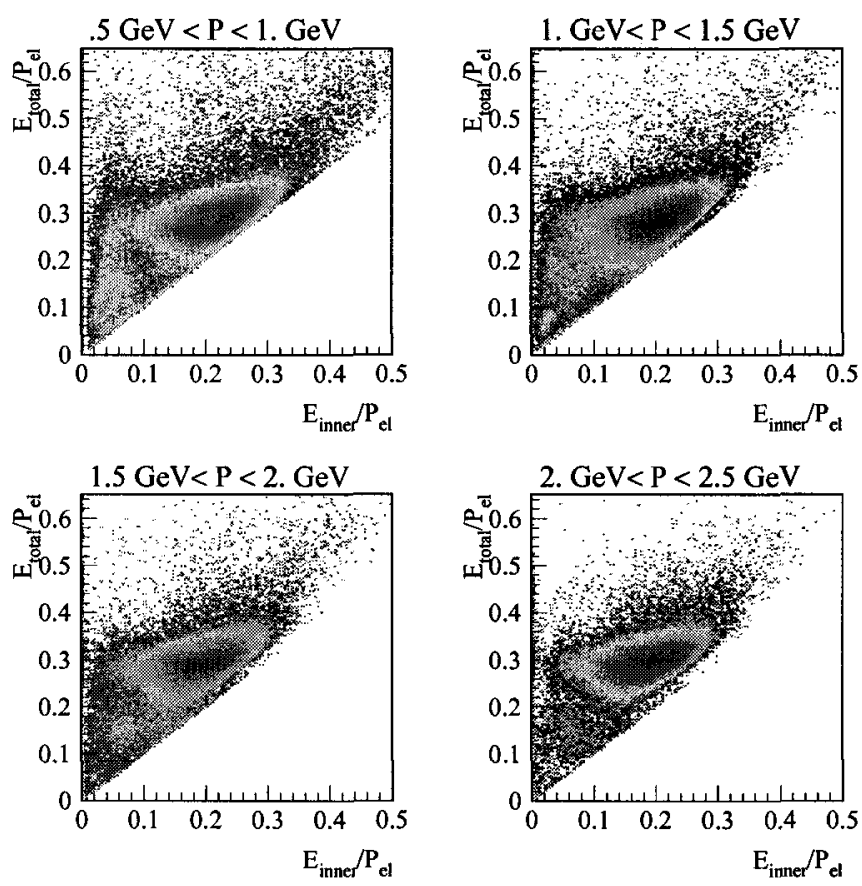

FIG. 65. $E_{\text {total }} / P_{e l}$ vs $E_{\text {inner }} / P_{e l}$ for different momentum bins. The concentration of events in the left bottom corner are pions.

pions in the inner part compared to the total energy they deposit in the calorimeter is approximately given by,

$$
E_{\text {inner }}=\frac{5}{13} E_{\text {total }}
$$

All these signature characteristics of the calorimeter which are unique for pions and electrons can therefore be used to separate them. Fig. 65 shows $E_{t o t a l} / P_{e l}$ vs $E_{i n n e r} / P_{e l}$ for different momentum bins. The concentration of events in the lower left corner of each plot are pions and are clearly separated from electrons, which are the events concentrated at $E_{\text {total }} / P_{e l} \approx 0.3$. From the plot it can clearly be seen that the separation between electrons and pions become more evident with increasing momentum, since for pions $E_{i n n e r} / P_{e l}$ and $E_{t o t a l} / P_{e l}$ decrease with increasing momentum while $E_{t o t a l} / P_{e l}$ remains the same for electrons. To reject pions the following cuts were applied (see Fig. 66,

- For the 5.x GeV data $\left(\mathrm{P}_{\mathrm{el}}<3 \mathrm{GeV}\right)$ events with $E_{\text {inner }} / P_{e l}>0.08$ and $E_{t o t a l} / P_{e l}>0.2$ were accepted (Fig. 66 (b) and (c)).

- For the 5.x GeV data $\left(\mathrm{P}_{\mathrm{el}}>3 \mathrm{GeV}\right)$ events with $E_{\text {inner }} / P_{\text {el }}>0.06$ and 
$E_{\text {total }} / P_{e l}>0.24$ were accepted (Fig. 66 (b) and (c)).

- For the $1.6 \mathrm{GeV}$ inbending data events with $E_{\text {inner }} / P_{e l}>0.08$ and $E_{\text {total }} / P_{e l}>$ $0.27-0.071 / \sqrt{P_{e l}}$ were accepted (Fig. 66(a)).

- For the $1 . \mathrm{x} \mathrm{GeV}$ outbending data $E_{\text {inner }} / P_{e l}>0.08$ and a sector and run dependent $E_{\text {total }} / P_{e l}$ cut which has the same functional form as for the 1.6 $\mathrm{GeV}$ inbending data were used.
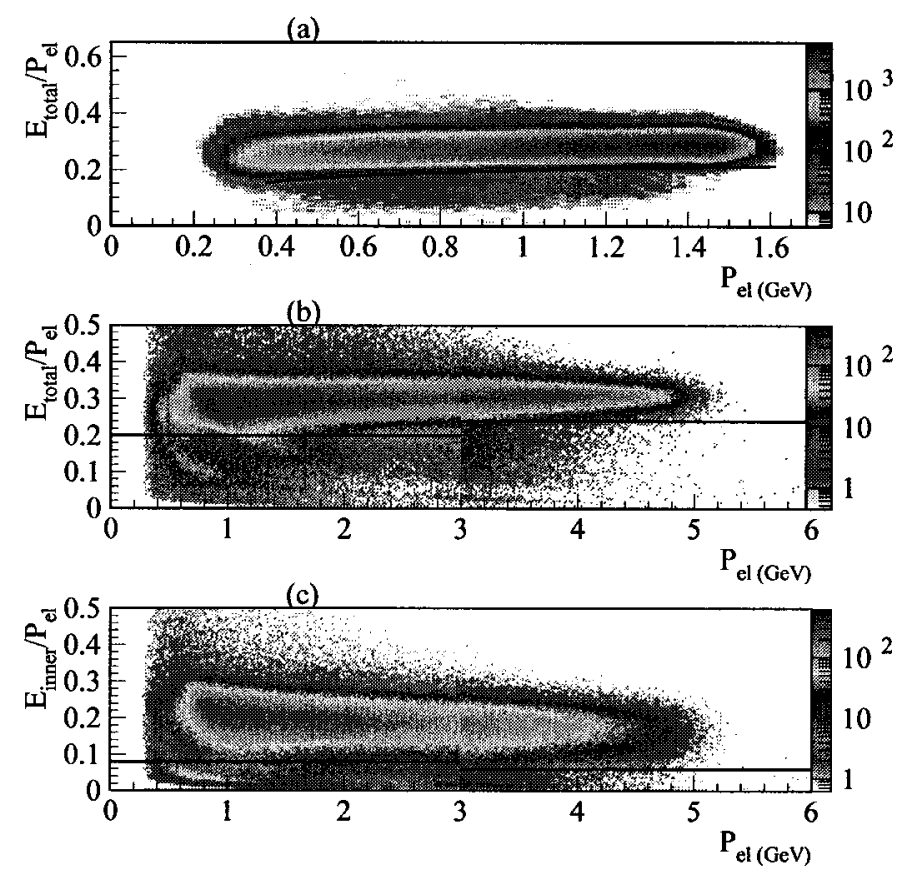

FIG. 66. Electromagnetic calorimeter cuts. (a) $E_{\text {inner }} / P_{e l}$ cut applied to the $1.6 \mathrm{GeV}$ inbending data, (b) $E_{\text {total }} / P_{e l}$ cuts applied to the $5 . \mathrm{x} \mathrm{GeV}$ data and (c) $E_{\text {inner }} / P_{e l}$ cuts applied to the $5 . \mathrm{x} \mathrm{GeV}$ data.

\subsubsection{Raster Correction and Vertex Cut}

A vertex cut is required to remove events which may have scattered from materials other than the target cell. To get a better vertex resolution the ADC values of the current that went into the raster magnet which produced a slow spiral motion of the beam were calibrated [54]. The $\mathrm{z}$ vertex position was corrected by adding the $\mathrm{z}$ component of the distance along the track length that was not taken into account in tracking,

$$
z_{\text {corr }}=z_{\text {meas }}+\Delta_{x y} / \tan (\theta),
$$


where $z_{\text {meas }}$ is the vertex position calculated by the tracking code and $\theta$ is the angle that the track makes with the beam line. The value $\Delta_{x y}$ is related to the raster $x$ and $y$ positions which are given in $\mathrm{cm}$ by,

$$
\Delta_{x y}=\left[x \cos \left(\phi_{s}\right)+y \sin \left(\phi_{s}\right)\right] / \cos \left(\phi-\phi_{s}\right),
$$

where $\phi$ and $\phi_{s}$ are the azimuthal angle of the track at the vertex and the sector angle in degrees. The raster $x$ and $y$ positions in centimeters were obtained by assuming that the raster $\operatorname{ADC} X$ and $Y$ values are linearly related to $x$ and $y$,

$$
\begin{aligned}
& x=\left(X-X_{0}\right) * c_{x}, \\
& y=\left(Y-Y_{0}\right) * c_{y} .
\end{aligned}
$$

The values $X_{0}, Y_{0}, c_{x}$ and $c_{y}$ for each run were determined by minimizing the $\chi^{2}$
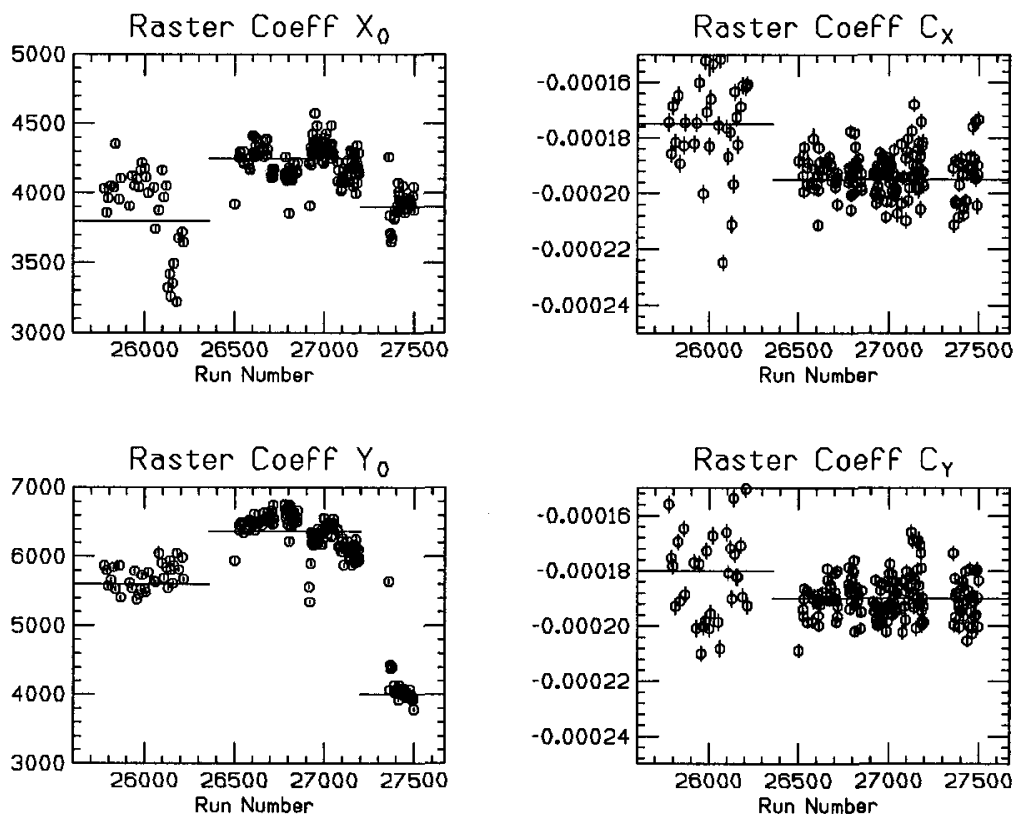

FIG. 67. The fit to the coefficients $X_{0}, Y_{0}, c_{x}$ and $c_{y}$ as a function of the run number [54]. The lines are the fit to each group of runs which have approximately the same number for each of the coefficients.

defined by the following equation,

$$
\chi^{2}=\sum_{1}^{N}\left(z_{\text {corr }}-z_{0}\right)^{2},
$$

where $z_{0}$ is another fit parameter which defines the center of the target. A fit to each group of runs was done to obtain an average value for each parameter (Fig. 67). 
This average value of each coefficient was used in the analysis to correct that group of runs.

Since we are now assuming that the particles have traveled a different track length than that assumed by the tracking code, in addition to the corrections discussed above, the $\phi$ angle also had to be corrected. This was corrected using,

$$
\phi_{c}=\phi_{0}-(q)(50)\left(\Delta_{x y} / 100\right) / 33.356 / P_{t r a}
$$

where $q$ is the charge of the particle, $P_{t r a}$ is the transverse momentum of the particle in $\mathrm{GeV}$ and the numbers 50, 100 and 33.356 are the magnetic field in $\mathrm{kG}$, a factor to convert $\mathrm{cm}$ to $\mathrm{m}$ and the inverse speed of light in appropriate units respectively.
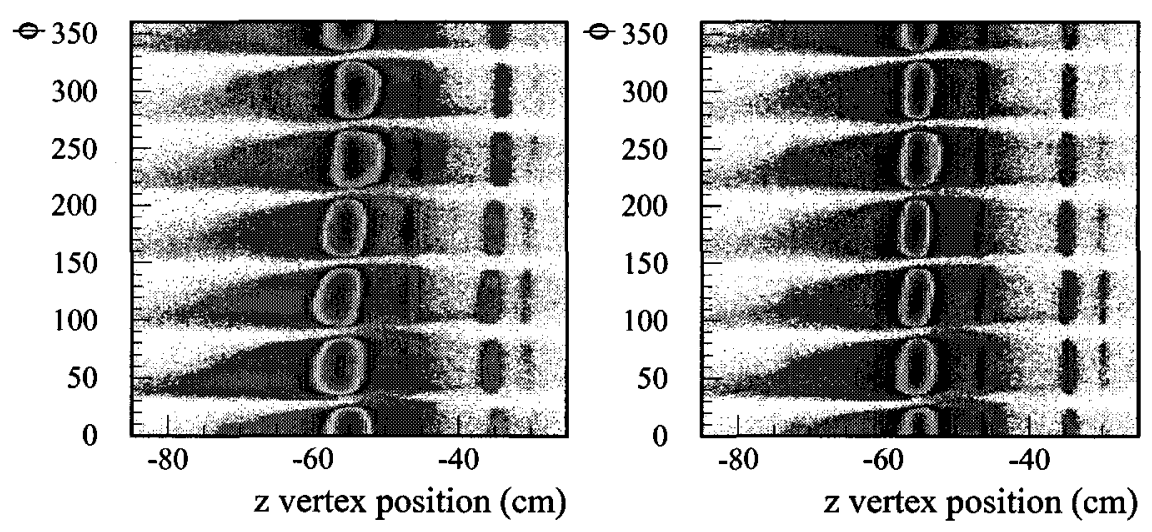

FIG. 68. The figure shows the $\phi$ angle vs the $\mathrm{z}$ vertex position before (left) and after (right) applying the raster correction. Data are $1.606 \mathrm{GeV}$ inbending data.

Fig. 68 shows the $\mathrm{z}$ vertex position before and after applying the correction. It can clearly be seen that the correction provided a better vertex resolution and removes the phi dependence of the $z$ position. We used a cut of $-58<z_{\text {vertex }}<-52$ after applying the correction to reject events scattered from upstream and downstream windows (Fig. 69).

\subsection{MOMENTUM CORRECTION}

Once the electrons are properly identified the next step is to construct the invariant mass spectrum for each kinematic bin. Since $W$ is an invariant quantity it should not show any kinematic dependence. However a $\theta$ and a $\phi$ (Fig. 70) dependence was observed in the $W$ spectrum because of systematic deviations of the particle momenta reconstructed by the tracking code. These deviations have been studied 


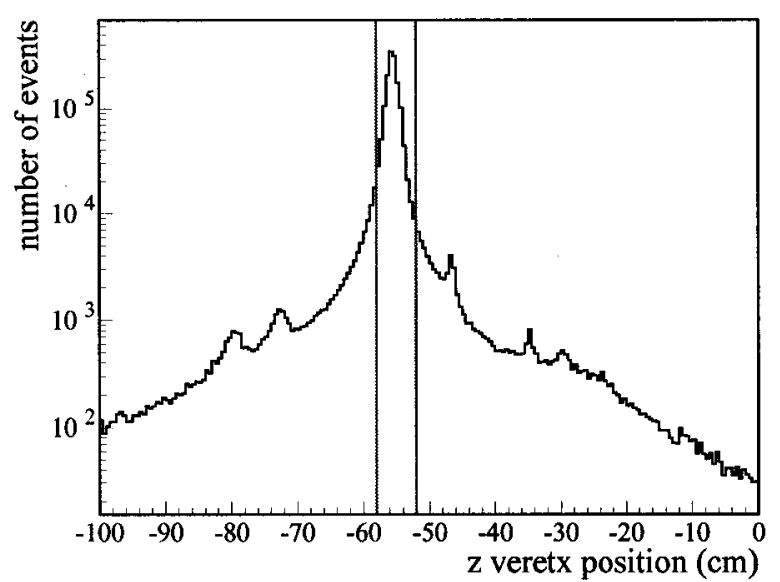

FIG. 69. After applying the raster correction a cut indicated by the two vertical lines was applied to select electrons scattered from the target. Data are $5.627 \mathrm{GeV}$ inbending data.

and understood to be due to a misalignment of the drift chambers relative to their nominal positions, different factors affecting the drift chamber wire position such as wire sag, incorrect information about drift chambers in the track reconstruction code and incomplete knowledge of the torus magnetic field distribution. Different CLAS analyses have been using different methods to take these effects into account; we used a method developed by the E6 run group. Details about this method are described in the CLAS note 2003-005 [55]. The method, which assumes that the deviations observed in the momentum are due to drift chamber displacements and incorrect values of the magnetic field in the track reconstruction code, corrects all momenta and all polar angles. To correct for the drift chamber position the displacement of a given reconstructed track is formalized as a change in the polar scattering angle $(\Delta \theta)$ and a change in the momentum $(\Delta p)$,

$$
\Delta \theta=(A+B \phi) \frac{\cos \theta}{\cos \phi}+(C+D \phi) \sin \theta
$$

and

$$
\frac{\Delta p}{p}=\left((E+F \phi) \frac{\cos \theta}{\cos \phi}+(G+H \phi) \sin \theta\right) \frac{p}{q B_{\text {Torus }}}
$$

where,

$$
\begin{aligned}
& B_{\text {Torus }}=0.76 \frac{I_{\text {Torus }} \sin ^{2} 4 \theta}{3375 \theta / r a d} \text { for } \theta<\pi / 8 \\
& B_{\text {Torus }}=0.76 \frac{I_{\text {Torus }}}{3375 \theta / \text { rad }} \text { for } \theta \geq \pi / 8
\end{aligned}
$$



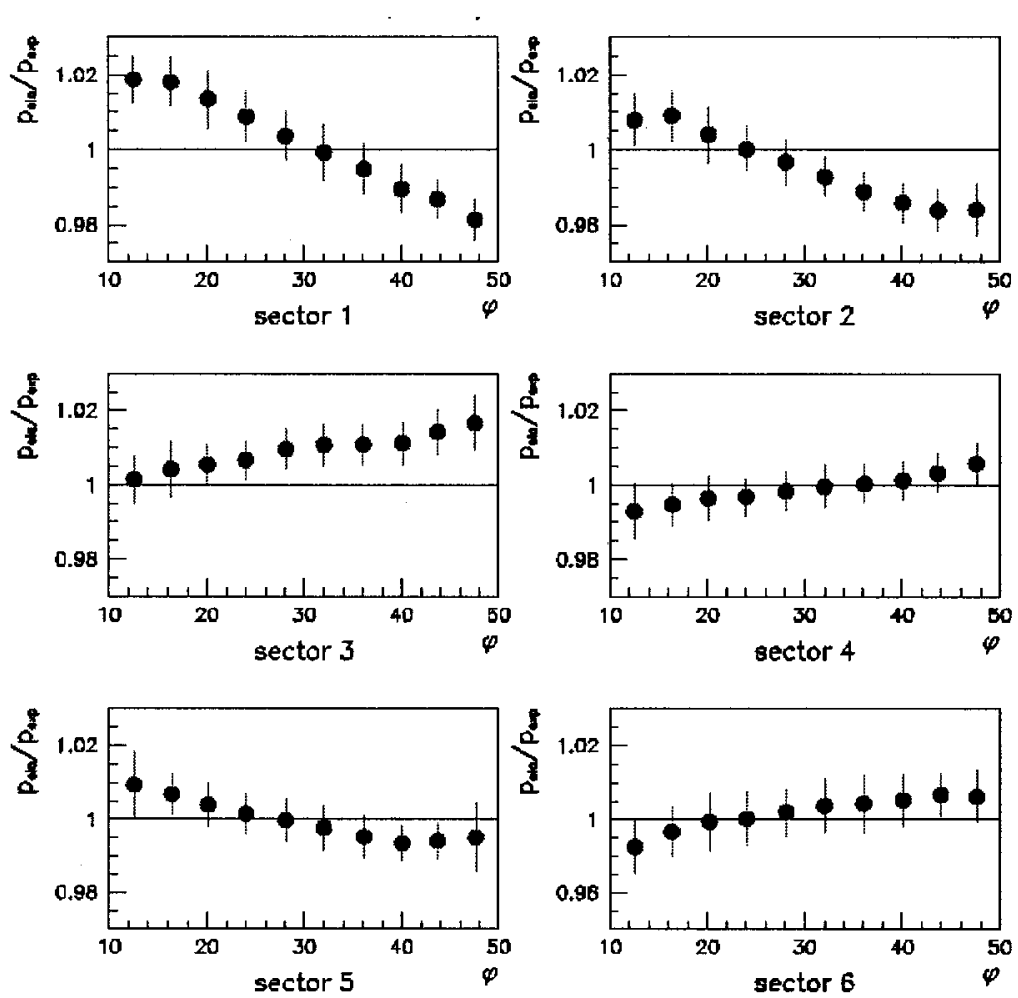

FIG. 70. The nominal value of the elastic peak position divided by the observed value as a function of the azimuthal angle $\phi$ for all six sectors [52]. The $\phi$ dependence of the ratio is clearly visible.

The constants $A$ through $H$ take into account drift chamber displacements along and away from the beam line, a phi dependent displacement and a $x$ dependent displacement for region 2 and 3 . The difference between the magnetic field map used in the track reconstruction code and the actual magnetic field distribution was corrected using the function,

$$
f=(J \cos (\theta)+K \sin (\theta)+L \sin (2 \theta))+(M \cos (\theta)+N \sin (\theta)+O \sin (2 \theta)) \phi
$$

Since the method is based on 4-momentum conservation a sample of elastic $p(e, e / p)$ events were used to determine fit parameters. The electrons were chosen using electron cuts and fiducial cuts described in the previous sections and the protons were chosen using the nominal reconstruction particle ID. To select elastic $p(e, e / p)$ events the following cuts were applied: $-1<\left|\phi_{e}-\phi_{p}\right|-180<1, \mid E($ miss $) \mid \leq 0.1 \mathrm{GeV}$, $\mid P_{z}$ (miss) $|\leq 0.1 \mathrm{GeV},| P_{x}$ (miss) $\mid \leq 0.07 \mathrm{GeV}$ and $\mid P_{y}$ (miss) $\mid \leq 0.07 \mathrm{GeV}$ (Fig. 71). The events so selected were then used to determine the fit parameters. Technical details about how the fit was done and how it was applied to correct the momentum 

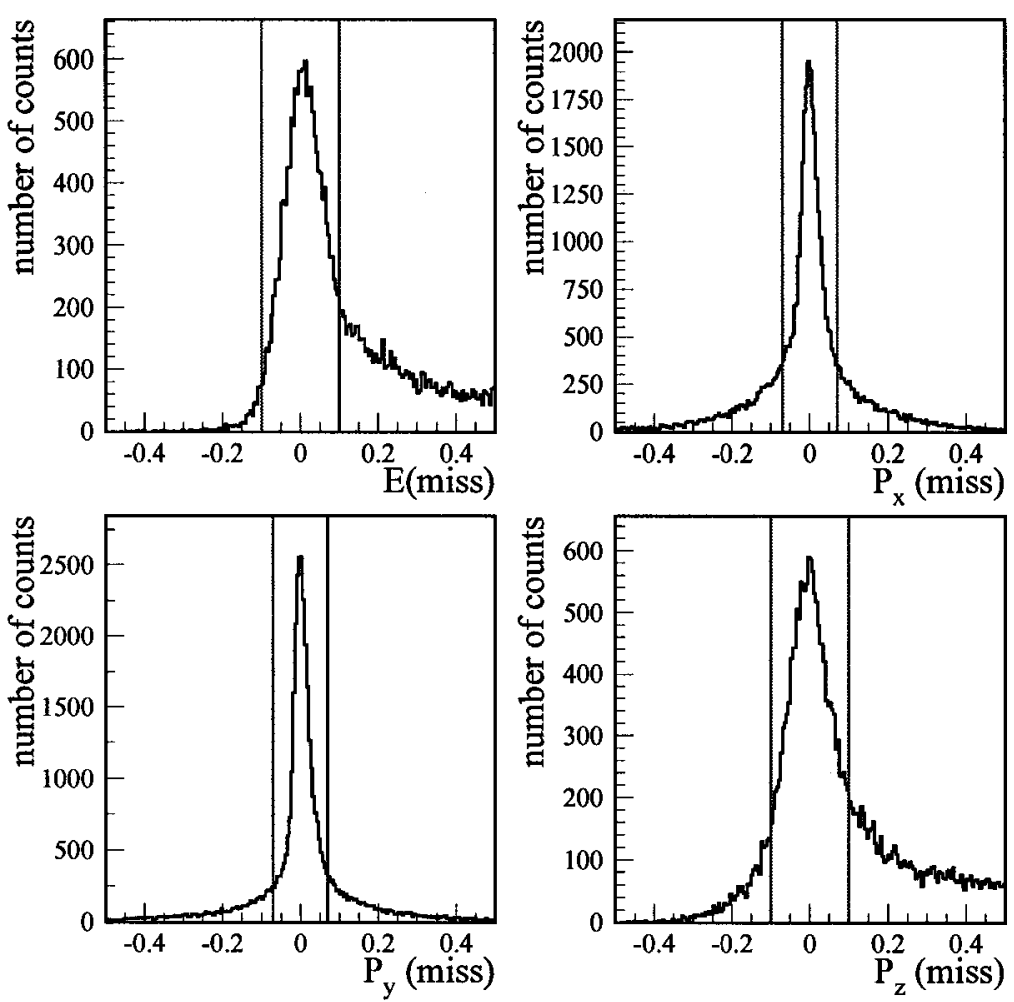

FIG. 71. The missing four momenta of events within $-1<\left|\phi_{e}-\phi_{p}\right|-180<1$. The vertical lines indicate the cuts applied to select elastic $p(e, e / p)$ events which were used in the fitting program to determine the fit parameters.

is described in the reference [55]. Fig. 72 shows the $W$ spectra for the $5.627 \mathrm{GeV}$ inbending data before and after applying the correction and Fig. 73 shows the phi dependence of the nominal value of the elastic peak position divided by the observed value after applying the correction. The correction improved the resolution of the elastic peak and also removed the phi dependence.

\subsection{EXTRACTION OF THE ASYMMETRY $A_{\|}$}

In experiments the asymmetry given in equation (16) is measured by observing the scattering rates since in an asymmetry analysis all the terms in the cross section except the rates cancel out leaving an asymmetry of rates. After taking into account the fact that the beam polarization, $P_{b}$, and the target polarization, $P_{t}$, are not $100 \%$ and correcting for unpolarized materials in the target, $F_{D F}$, the measured asymmetry 

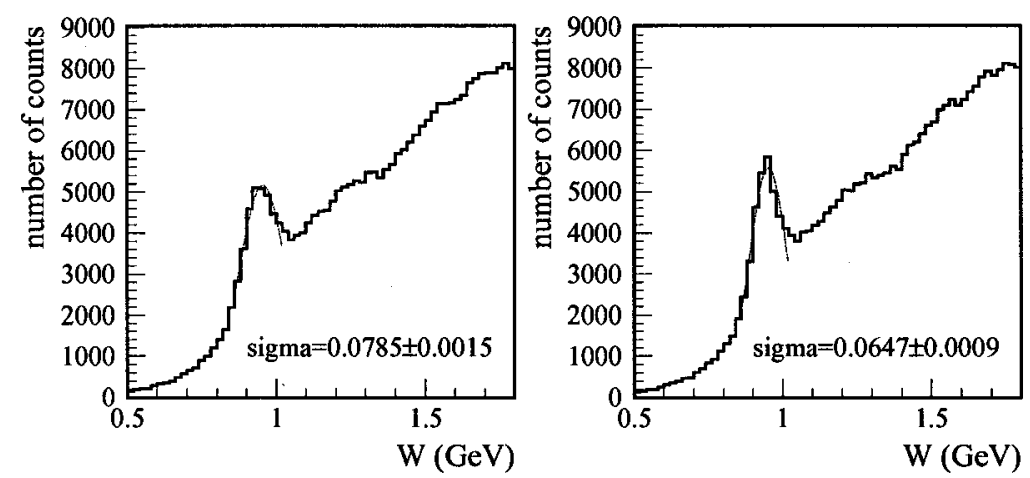

FIG. 72. W distribution for inclusive electron scattering before (left) and after applying the momentum correction (right). The data are $5.627 \mathrm{GeV}$ inbending data and the numbers quoted for sigma are in $\mathrm{GeV}$.
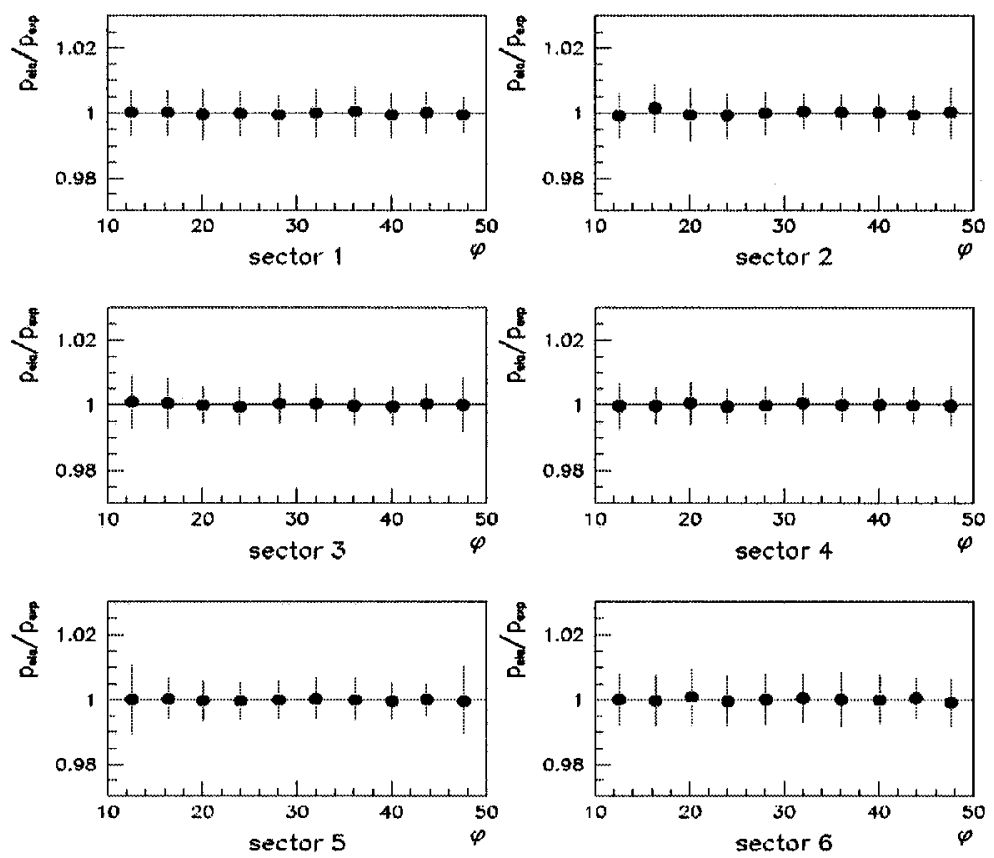

FIG. 73. Same as Fig. 70 but after applying the momentum correction [52]. 
$A_{\|}^{\text {meas }}$ can be written as,

$$
A_{\|}^{\text {meas }}=\frac{C_{b a c k}}{P_{b} P_{t} F_{D F}}\left(\frac{n^{-}-n^{+}}{n^{-}+n^{+}}\right)
$$

where $n^{+}\left(n^{-}\right)$are the raw counting rates normalized to the accumulated beam charge for beam helicity parallel (antiparallel) to the target spin, and $C_{b a c k}$ is a correction due to the presence of electrons which are coming from pair symmetric processes and pions in the electron sample. In addition the radiative effects, which are the corrections to the first order Feynman diagram shown in Fig.1, also have to be taken into account. In this section each of these terms will be discussed in detail.

\subsubsection{Raw Asymmetry}

The raw asymmetry, $A_{\text {raw }}$, is the asymmetry due to raw counting rates,

$$
A_{\text {raw }}=\frac{n^{-}-n^{+}}{n^{-}+n^{+}}
$$

To obtain the raw asymmetry, $A_{\text {raw }}$, the helicity state of each event has to be determined carefully. The helicity sequence of the beam delivered to the hall is formed by pairs of electron helicity states (original and the compliment) that are opposite in sign. The original helicity state was pseudo-randomly selected at the injector and the corresponding helicity complement state always follows the original state. The flipping of the helicity was done at a $30 \mathrm{~Hz}$ rate and the readout of the integrated beam charge for a given helicity state was stored in the data stream immediately after a helicity flip. A "Sync" pulse with twice the frequency of the helicity pulse was delivered to the hall and was recorded in the data stream along with the helicity information which was later used in identifying the helicity flips (Fig. 74).

The helicity states recorded by the data acquisition system sometimes failed due to dead time problems. Therefore it is possible to have broken sequences which would result in unpaired helicity states. These unpaired helicity states can introduce a false asymmetry. Therefore a program was developed to match the original helicity with its complement and to throw away any unpaired helicity states.

We also had to be careful not to integrate the beam charge when the data acquisition system fails to record events. This problem was avoided by gating the beam charge with the data acquisition system such that the beam charge would not be integrated during dead times. 
Sync bit

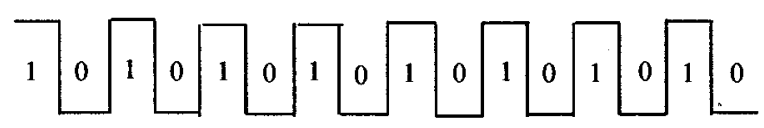

Helicity bit

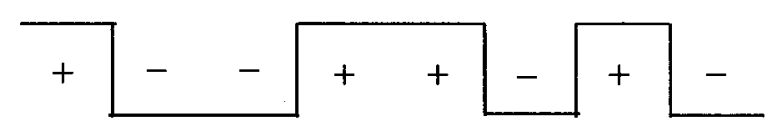

Original/Complement state

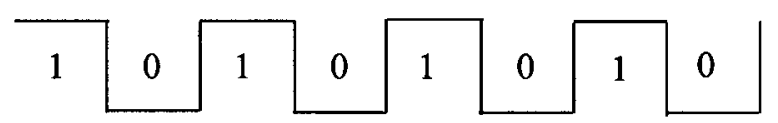

FIG. 74. The structure of the beam helicity and the sync. The original and the complement states are identified by matching the sync and the helicity sequence. More details are in the text.

Once the helicities are paired, the normalized counts for a given helicity state can be obtained by simply taking the number of counts for that helicity state and then dividing it by the respective "gated" integrated beam charge.

\subsubsection{Dilution Factor}

In this experiment we are interested only in scattering from the polarized deuterium. Therefore to extract asymmetries it is necessary to remove the events scattered from the unpolarized target materials. The fraction of events scattered from the polarized deuterium in the target is known as the dilution factor,

$$
F_{D F}=\frac{N_{N D_{3}}-N_{b a c k}}{N_{N D_{3}}}
$$

where $N_{\mathrm{ND}_{3}}$ and $N_{b a c k}$ are the number of counts scattered from the $\mathrm{ND}_{3}$ target and the nondeuteron materials in the target. The $\mathrm{z}$ vertex cut only removes the scattering contribution from the external heat shield and the cryostat windows. However other materials such as ${ }^{15} \mathrm{~N}$, helium, foils and target windows also have to be removed. The best possible way to remove the ${ }^{15} \mathrm{~N}$ contribution from $\mathrm{ND}_{3}$ is by taking ${ }^{15} \mathrm{~N}$ data at the exact beam settings as $\mathrm{ND}_{3}$. However due to technical difficulties taking ${ }^{15} \mathrm{~N}$ data in between $\mathrm{ND}_{3}$ runs was not an option. Therefore a material which has 
TABLE IV. Target parameters.

\begin{tabular}{|l|c|}
\hline Item & Value \\
\hline$f$ & 0.177 \\
\hline$\rho_{H e}$ & $0.145 \mathrm{~g} / \mathrm{cm}^{3}=0.0362 \mathrm{~mol} / \mathrm{cm}^{3}$ \\
\hline$L$ & $1.9 \mathrm{~cm}$ \\
\hline$\rho_{C}$ & $2.17 \mathrm{~g} / \mathrm{cm}^{3}=0.180 \mathrm{~mol} / \mathrm{cm}^{3}$ \\
\hline$l_{C}$ & $0.23 \mathrm{~cm}$ \\
\hline$\rho_{N}$ & $1.1 \mathrm{~g} / \mathrm{cm}^{3}=0.07325 \mathrm{~mol} / \mathrm{cm}^{3}$ \\
\hline$l_{N}$ & $0.65 \mathrm{~cm}$ \\
\hline$\rho_{A}$ & $1.056 \mathrm{~g} / \mathrm{cm}^{3}=0.0502 \mathrm{~mol} / \mathrm{cm}^{3}$ \\
\hline
\end{tabular}

a structure close to ${ }^{15} \mathrm{~N},{ }^{12} \mathrm{C}$, was chosen instead. To remove the contribution from the ${ }^{15} \mathrm{~N}$ in ammonia as well as the helium and foils in the target, data on ${ }^{12} \mathrm{C}$ and ${ }^{4} \mathrm{He}$ were taken at all beam energies. However scattering from ${ }^{12} \mathrm{C}$ is not identical to scattering from ${ }^{15} \mathrm{~N}$ due to the different number of nucleons in the two materials and the extra neutron in ${ }^{15} \mathrm{~N}$, to address this problem we were able to take data on a solid ${ }^{15} \mathrm{~N}$ target at some beam energies. Therefore the ${ }^{15} \mathrm{~N}$ spectrum was simulated using ${ }^{12} \mathrm{C}$ data by parameterizing the ${ }^{15} \mathrm{~N}$ cross section as a function of the ${ }^{12} \mathrm{C}$ cross section. In this section the procedure developed to simulate the ${ }^{15} \mathrm{~N}$ background and extract the dilution factor will be discussed.

\section{Fit to ${ }^{15} \mathrm{~N}$ data}

To create an accurate background spectrum, the ${ }^{15} \mathrm{~N}$, foils and helium have to be taken into account. Although the carbon target was constructed to have the same external foils and window thicknesses and total radiation lengths as the $\mathrm{ND}_{3}$ target, there was more helium in the carbon target than in the $\mathrm{ND}_{3}$ target since carbon is more dense than ammonia. Therefore the correct amount of helium in each target has to be determined using other known quantities. As a first step the normalized counts for all four targets, carbon $\left(n_{C}\right)$, Helium $\left(n_{M T}\right)$, nitrogen $\left(n_{N}\right)$ and $\mathrm{ND}_{3}\left(n_{A}\right)$ can be written as sums of contributions from entrance and exit foils $(F)$, Helium 
$(H e)$, carbon $(C)$, nitrogen $(N)$ and deuterium [56],

$$
\begin{aligned}
& n_{M T}=\left(\rho_{F} l_{F} \frac{\sigma_{F}}{\sigma_{D}}+\rho_{H e} L \frac{\sigma_{H e}}{\sigma_{D}}\right) F \sigma_{D}=\left(\rho_{C} l_{C} f \frac{\sigma_{C}}{\sigma_{D}}+\rho_{H e} L \frac{\sigma_{H e}}{\sigma_{D}}\right) F \sigma_{D}, \\
& n_{C}=\left(\rho_{C} l_{C}(1+f) \frac{\sigma_{C}}{\sigma_{D}}+\rho_{H e}\left(L-l_{C}\right) \frac{\sigma_{H e}}{\sigma_{D}}\right) F \sigma_{D}, \\
& n_{N}=\left(\rho_{C} l_{C} f \frac{\sigma_{C}}{\sigma_{D}}+\rho_{H e}\left(L-l_{N}\right) \frac{\sigma_{H e}}{\sigma_{D}}+\rho_{N} l_{N} \frac{\sigma_{N}}{\sigma_{D}}\right) F \sigma_{D}, \\
& n_{A}=\left(\rho_{C} l_{C} f \frac{\sigma_{C}}{\sigma_{D}}+\rho_{H e}\left(L-l_{A}\right) \frac{\sigma_{H e}}{\sigma_{D}}+\rho_{A} l_{A}\left[\frac{\sigma_{N}}{\sigma_{D}}+3\right]\right) F \sigma_{D},
\end{aligned}
$$

where $\rho$ is the density in mol per $\mathrm{cm}^{3}$ and $l$ the length of each component. The cross sections are in $\mathrm{cm}^{2}$ per nucleus. The factor $F$ contains all conversion factors and the acceptance and overall efficiency of CLAS at a given kinematic point and $f$ is the contribution to the count rate from all foils combined expressed as a fixed fraction of the contribution from ${ }^{12} \mathrm{C}$ in the carbon target. Table IV lists the values of these constants. The physical length of the target, $L$, which is the distance between the banjo entrance and exit window can also be extracted by doing a fit to $r=n_{M T} / n_{C}$ in the $1.5 \mathrm{GeV}<W<2 \mathrm{GeV}$ region and using the relation,

$$
L=\left(\frac{3 \rho_{C} l_{C}[(1+f) r-f]}{\rho_{H e}}-r l_{C}\right) /(1-r),
$$

in which we assume that $\sigma_{C}=3 \sigma_{\mathrm{He}}$. Figure 75 shows $r$ extracted using data for one $Q^{2}$ bin. The number obtained for $L$ using this value agrees well with the physical target measurements.

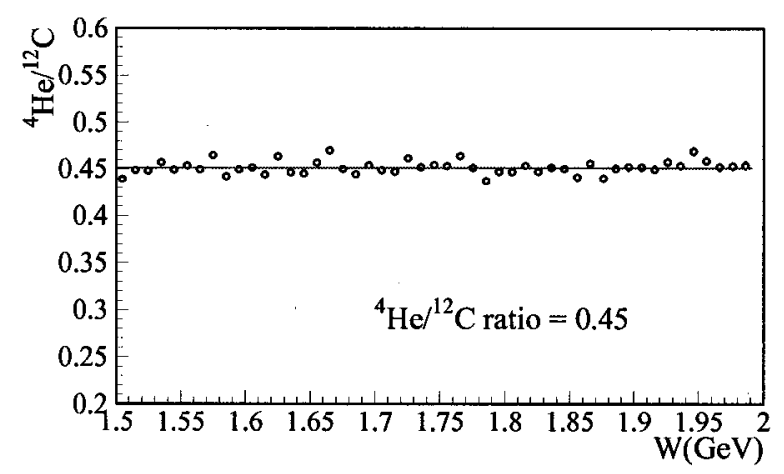

FIG. 75. The ratio of normalized ${ }^{4} \mathrm{He}$ and ${ }^{12} \mathrm{C}$ spectra for one $Q^{2}$ bin at the beam energy $5.6 \mathrm{GeV}$. The average value of the ratio is approximately equal to .45 which gives a target length, $L$, of $1.9 \mathrm{~cm}$.

Using equations (147) and (148) normalized counts from the ${ }^{12} \mathrm{C}$ slab only, $\rho_{C} l_{C} \sigma_{C}=n_{12}^{\prime}$, and the counts per $1 \mathrm{~cm}$ length of liquid ${ }^{4} \mathrm{He}, \rho_{\mathrm{He}} \sigma_{\mathrm{He}}=n_{4 \mathrm{He}}^{\prime}$, 
can be obtained,

$$
\begin{aligned}
& n_{{ }_{12} C}^{\prime}=\frac{L}{L+f l_{C}} n_{C}-\frac{L-l_{C}}{L+f l_{C}} n_{M T}, \\
& n_{4_{H e}}^{\prime}=\frac{1+f}{L+f l_{C}} n_{M T}-\frac{f}{L+f l_{C}} n_{C} .
\end{aligned}
$$

The nitrogen target spectrum can then be expressed using $n_{{ }_{12} C}^{\prime}$ and $n_{4 \mathrm{He}}^{\prime}$ as,

$$
n_{N}=f n_{12}^{\prime} C+\left(L-l_{N}\right) n_{4 H e}^{\prime}+l_{N} \rho_{N} \frac{\sigma_{N}}{\sigma_{D}} F \sigma_{D} .
$$

Further, assuming that the ${ }^{15} \mathrm{~N}$ cross section can be parameterized using ${ }^{12} \mathrm{C}$ cross section as,

$$
\sigma_{N}=\left(a+b \frac{\sigma_{n}}{\sigma_{D}}\right) \sigma_{C}
$$

equation (155) can be written as,

$$
n_{N}=n_{M T}-l_{N} n_{4}^{\prime}{ }_{H e}+\frac{l_{N} \rho_{N}}{l_{C} \rho_{C}}\left(a+b \frac{\sigma_{n}}{\sigma_{D}}\right) n_{{ }_{12} C}^{\prime} .
$$

Ideally,

$$
\sigma_{N}=\left(\frac{7 \sigma_{D}+\sigma_{n}}{6 \sigma_{D}}\right) \sigma_{C},
$$

which gives $a=7 / 6$ and $b=1 / 6$. Since we have nitrogen data at some beam energies, parameters $a$ and $b$ were determined by minimizing the $\chi^{2}$ between the ${ }^{15} \mathrm{~N}$ spectrum constructed using equation (157), $n_{N}^{\text {sim }}$ and the real ${ }^{15} \mathrm{~N}$ spectrum, $n_{N}^{\text {real }}$ (Fig. 76),

$$
\chi^{2}=\sum_{i} \sum_{j}\left(n_{N}^{r e a l}-n_{N}^{s i m}\right)^{2},
$$

where $i$ and $j$ are the $W$ and $Q^{2}$ bins respectively. Table $\mathrm{V}$ lists the $a$ and $b$ values obtained using the $1.7 \mathrm{GeV}$ outbending and the $5.6 \mathrm{GeV}$ inbending ${ }^{15} \mathrm{~N}$ data.

Fig. 77 shows the carbon spectrum and the equivalent simulated nitrogen spectrum. It can clearly be seen that in the inelastic region there is a noticeable difference.

\section{Target length $l_{A}$}

The ammonia in the target, which is composed of small granules, cannot fill the entire space of the target cell. The length occupied by these ammonia beads, $l_{A}$, can be obtained using two different methods [56]. 
TABLE V. Summary of $a$ and $b$.

\begin{tabular}{|c|c|c|}
\hline Beam energy & $a$ & $b$ \\
\hline $1.7 \mathrm{GeV}$ & $1.3781 \pm 0.31947 E-02$ & $0.29700 \pm 0.91581 E-02$ \\
$5.6 \mathrm{GeV}$ & $1.1803 \pm 0.48557 E-02$ & $0.09000 \pm 0.96114 E-01$ \\
\hline
\end{tabular}

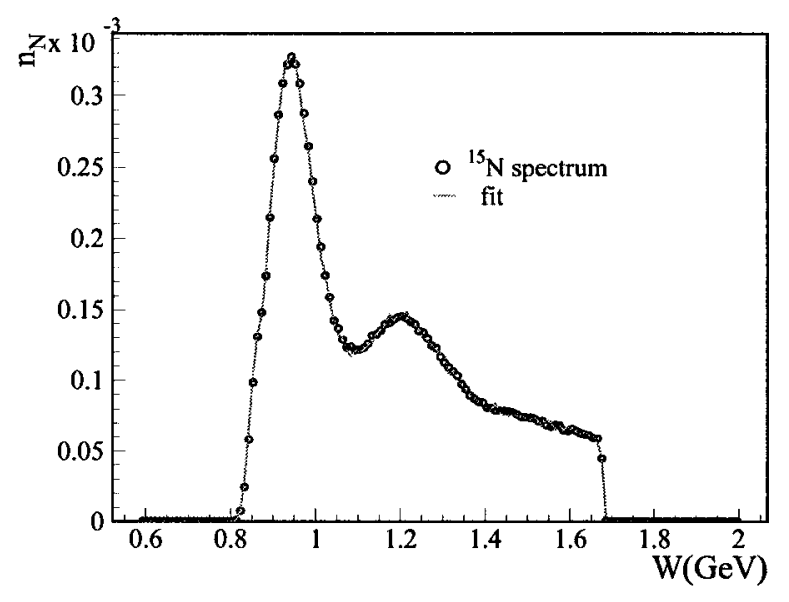

FIG. 76. To obtain parameters $a$ and $b$, a global fit to all $Q^{2}$ and $W$ bins was performed. The plot shows a comparison of the real ${ }^{15} \mathrm{~N}$ spectrum and the fit results for the $Q^{2}=0.14 \mathrm{GeV}^{2}$ bin. Details about the fit are given in the text.

In the first method the ammonia target spectrum and the combined ${ }^{12} \mathrm{C}$ and ${ }^{4} \mathrm{He}$ spectra are compared in the low- $\mathrm{W}$ tail $(W<0.9 \mathrm{GeV})$, where deuterium does not contribute. However for the deuteron there is a large momentum tail in the low-W region. Therefore it is difficult to determine a region of $W$ that is dominated by nondeuteronic material. Due to this uncertainty this method was not used to determine $l_{A}$.

The second method makes the assumption that the ratio of cross sections for different target materials in the high- $W$ region $(W>1.5 \mathrm{GeV})$ can be expressed in terms of the composite number of protons and neutrons in the material. Therefore $\sigma_{N D_{3}} / \sigma_{C}$ can be written as,

$$
\frac{\sigma_{N D_{3}}}{\sigma_{C}}=a+b \frac{\sigma_{n}}{\sigma_{D}}+3 \frac{\sigma_{D}}{\sigma_{C}}
$$




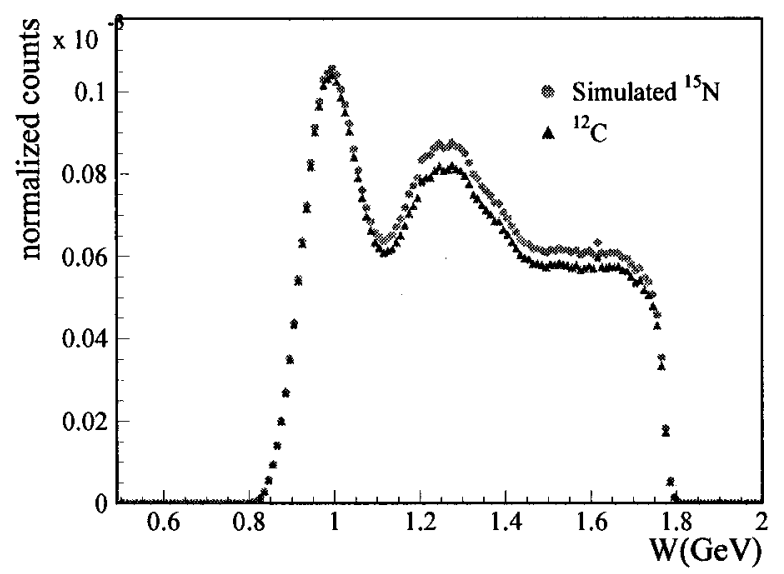

FIG. 77. Comparison of the simulated ${ }^{15} \mathrm{~N}$ spectrum and the ${ }^{12} \mathrm{C}$ spectrum.

and $n_{A}$ can be written as,

$$
\begin{array}{r}
n_{A}=\left(\left[\rho_{A} l_{A}\left(a+b \frac{\sigma_{n}}{\sigma_{D}}+3 \frac{\sigma_{D}}{\sigma_{C}}\right)+\rho_{C} l_{C} f\right] \frac{n_{12 C}^{\prime}}{\rho_{C} l_{C}}+\left(L-l_{A}\right) n_{4 H e}^{\prime}\right) \\
=n_{M T}+l_{A}\left(\left[\frac{\rho_{A}}{\rho_{C} l_{C}}\left(a+b \frac{\sigma_{n}}{\sigma_{D}}+3 \frac{\sigma_{D}}{\sigma_{C}}\right)\right] n_{{ }_{12} C}^{\prime}-n_{4_{H e}}^{\prime}\right) .
\end{array}
$$

Then $l_{A}$ can be extracted by further assuming that $\sigma_{D}=1 / 6 \sigma_{C}$,

$$
l_{A}=\left(n_{A}-n_{M T}\right) /\left(\left[\frac{\rho_{A}}{\rho_{C} l_{C}}\left(a+b \frac{\sigma_{n}}{\sigma_{D}}+0.5\right)\right] n_{1_{C} C}^{\prime}-n_{4}^{\prime} H e .\right.
$$

Fig. 78 shows $l_{A}$ for one $Q^{2}$ bin for the $5.6 \mathrm{GeV}$ data, which clearly is constant over the inelastic region.

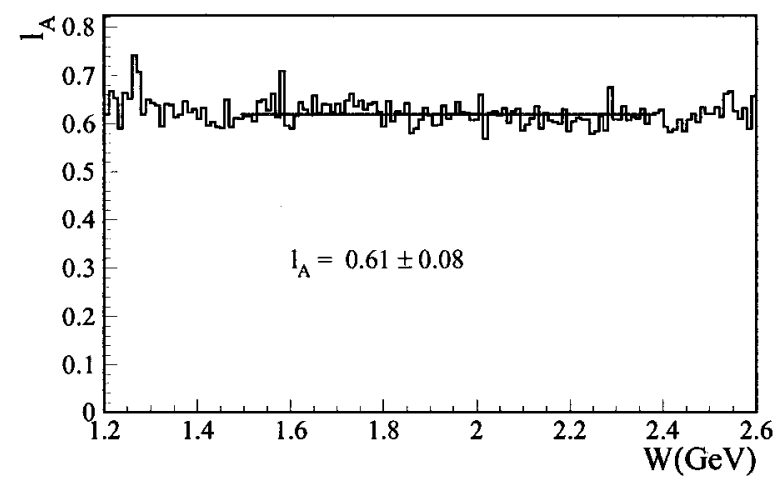

FIG. 78. The figure shows the target length $l_{A}$ vs $W . l_{A}$ is determined by doing a fit to the high $W$ region where it is constant. 

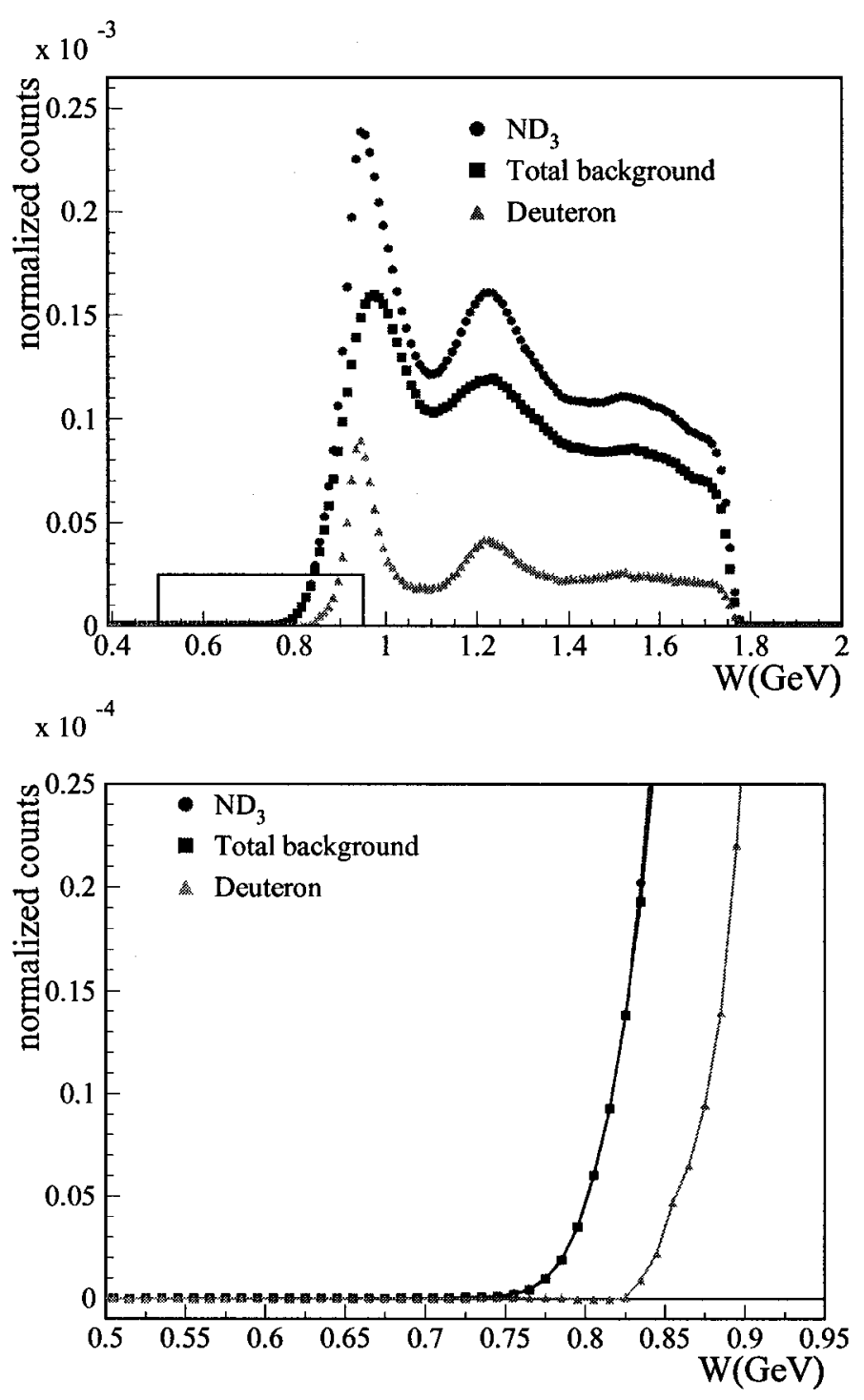

FIG. 79. The figure (top) shows the $\mathrm{ND}_{3}$ spectrum, the constructed total background and the deuteron spectrum. The bottom plot is focused on the the low $W$ tail outlined by the box on the top plot. From the plot it is evident that we are not under or over subtracting the background. 


\section{Background subtraction}

The non deuteronic target background, $n_{A-D}$, can be obtained by adding the simulated nitrogen, all the foils and the proper amount of helium in the ammonia target,

$$
\begin{aligned}
n_{A-D}= & \left(\left[\frac{\rho_{A} l_{A}}{\rho_{C} l_{C}}\left(a+b \frac{\sigma_{n}}{\sigma_{D}}\right)+f\right] n_{{ }_{12} C}^{\prime}+\left(L-l_{A}\right) n_{4_{H e}^{\prime}}^{\prime}\right) \\
& =n_{M T}+l_{A}\left(\left[\frac{\rho_{A}}{\rho_{C} l_{C}}\left(a+b \frac{\sigma_{n}}{\sigma_{D}}\right)\right] n_{{ }^{2} C}^{\prime}-n_{4 H e}^{\prime}\right) .
\end{aligned}
$$

Fig. 79 shows the $\mathrm{ND}_{3}$ spectrum, the total simulated background and the $\mathrm{D}_{3}$ spectrum. To make sure that we are not over or under subtracting the background, the low $\mathrm{W}$ tail of each $Q^{2}$ bin was also throughly checked. From the plots it is clear that the background simulated using this method is giving the correct background and matches very well with the low $\mathrm{W}$ tail of ammonia.

Once the background is subtracted the dilution factor can be determined using equation (146). To make sure there is no sector dependence, the dilution factor for each sector was compared for each $Q^{2}$ bin (Fig. 80).

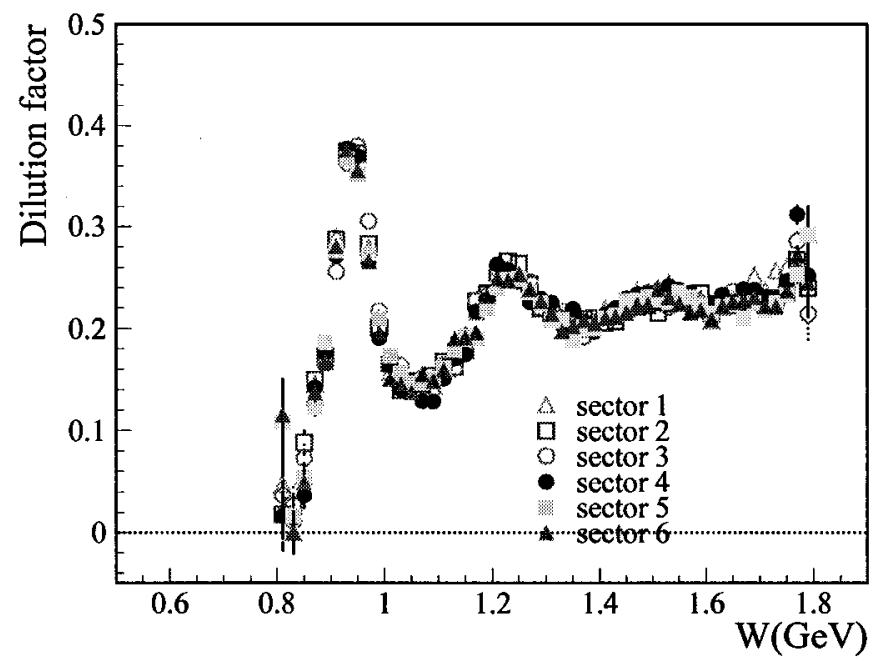

FIG. 80. The dilution factor for all six sectors for one $Q^{2}$ bin. The data are $1.6 \mathrm{GeV}$ inbending data.

Fig. 81 shows the ratio $\mathrm{ND}_{3} / \mathrm{C}$ calculated using models which include radiative corrections for both $\mathrm{C}$ and $\mathrm{ND}_{3}$. The ratio obtained using data shows large deviations from the calculated ratio at high $W$. The same behavior was also observed in the dilution factor measurements. Since the reasons for this behavior are not well understood and believed to be due to background processes such as pion contamination and pair symmetric processes, a $Q^{2}$ dependent $W$ cut was used to remove this 
region. Table VI lists the upper $\mathrm{W}$ limit selected for each $Q^{2}$ bin. When selecting

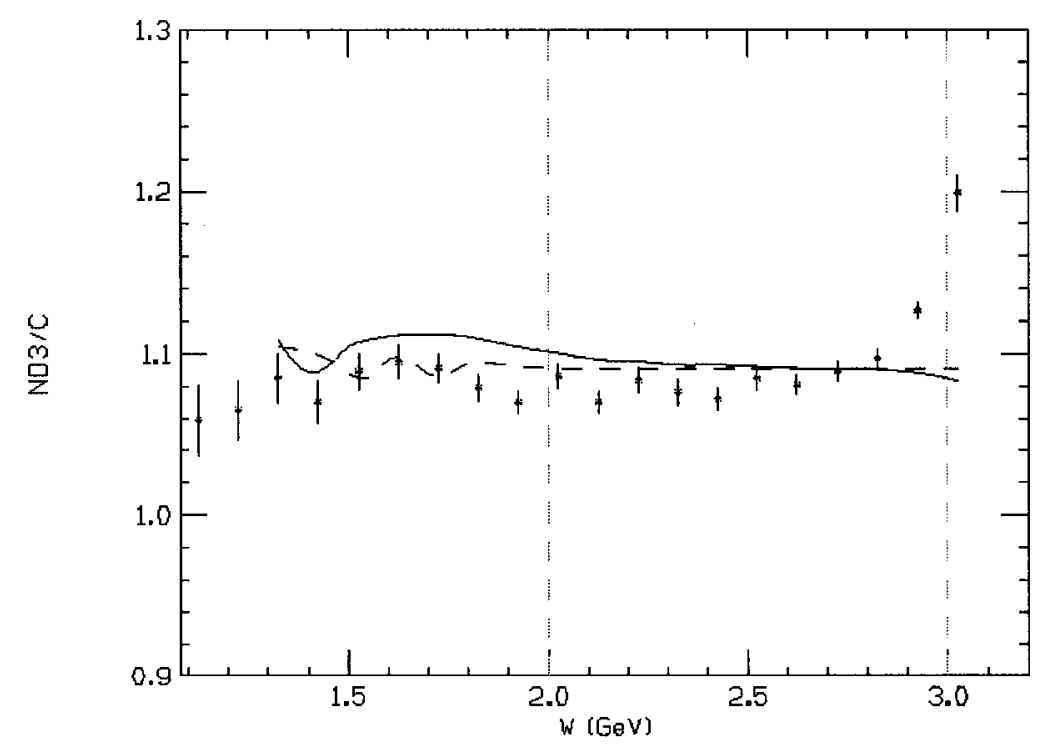

FIG. 81. The figure shows the $\mathrm{ND}_{3} /{ }^{12} \mathrm{C}$ ratio obtained using data and calculated using a model (dashed line) [57]. The solid line is the ratio calculated after applying radiative corrections. Notice the large deviations between the data and the calculated ratios in the high $W$ region.

this $W$ limit the contamination due to pair symmetric processes and pion contamination were also taken into account (more details in section 5.7.3). The high $W$ limit for each $Q^{2}$ bin was chosen as the lesser of the $W$ bin corresponding to a $20 \%$ contamination for pair symmetric processes or a $20 \%$ correction to pion contamination.

Fig. 82 shows the dilution factor for all $1.6 \mathrm{GeV} Q^{2}$ bins. From the plots it is evident that the dilution factor is not strongly $Q^{2}$ dependent.

For the $5 . \mathrm{x} \mathrm{GeV}$ data there was an additional problem in determining the dilution factor. Since the 5.x GeV data are divided among a large number of $\mathrm{W}$ bins, the dilution factor showed large statistical fluctuations from bin to bin. Therefore to determine the dilution factor of the $i^{\text {th }} \mathrm{W}$ bin and the $j^{\text {th }} Q^{2}$ bin, the counts for $\mathrm{ND}_{3}, n_{A}$, and the background, $n_{A-D}$, of the $(i, j)^{t h}$ bin were averaged as follows,

$$
\begin{aligned}
& n_{A}(i, j)=\sum_{j-1}^{j+1} \sum_{i-3}^{i+3} n_{A}(i, j) \\
& n_{A-D}(i, j)=\sum_{j-1}^{j+1} \sum_{i-3}^{i+3} n_{A-D}(i, j)
\end{aligned}
$$

The dilution factor obtained for some of the 5.x GeV settings are shown in Fig. 83. 
TABLE VI. The table lists the high $W$ cut applied to each $Q^{2}$ bin.

\begin{tabular}{|l|c|c|}
\hline beam energy & $Q^{2}\left(\mathrm{GeV}^{2}\right)$ & upper $W$ limit $(\mathrm{GeV})$ \\
\hline $1 . \mathrm{x} \mathrm{GeV}$ & 0.05 & 1.59 \\
& 0.06 & 1.59 \\
& 0.07 & 1.59 \\
& 0.084 & 1.59 \\
& 0.1 & 1.59 \\
& 0.12 & 1.59 \\
& 0.14 & 1.59 \\
& 0.17 & 1.59 \\
& 0.2 & 1.74 \\
& 0.24 & 1.74 \\
& 0.3 & 1.71 \\
& 0.35 & 1.67 \\
& 0.42 & 1.63 \\
& 0.5 & 1.60 \\
& 0.6 & 1.50 \\
\hline $5 . \mathrm{G} \mathrm{GeV}$ & 0.7 & 2.80 \\
& 0.84 & 2.80 \\
& 1.0 & 2.70 \\
& 1.2 & 2.70 \\
& 1.4 & 2.70 \\
& 1.7 & 2.60 \\
& 2.0 & 2.60 \\
& 2.4 & 2.56 \\
& 3.0 & 2.48 \\
& 3.5 & 2.35 \\
& 4.2 & 2.13 \\
\hline
\end{tabular}



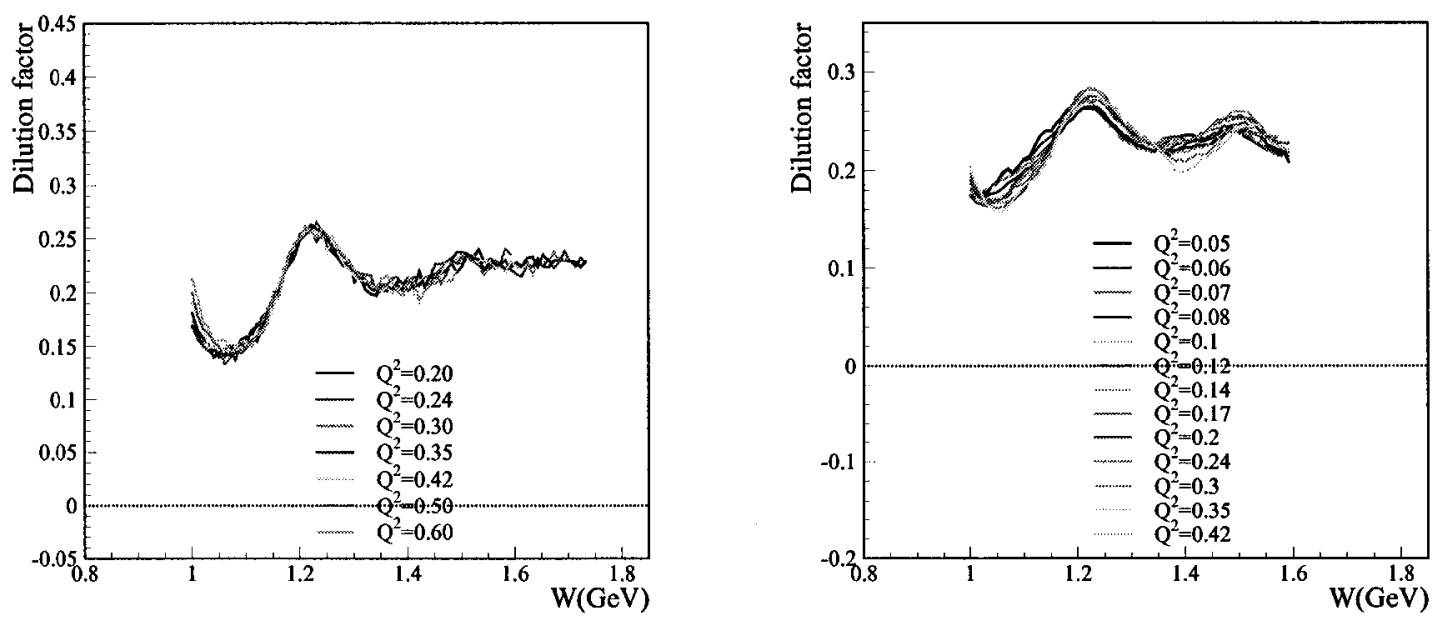

FIG. 82. The dilution factor plotted as a function of $W$ for $1.6 \mathrm{GeV}$ data. The left plot is for inbending torus setting and the right plot is for outbending torus setting.
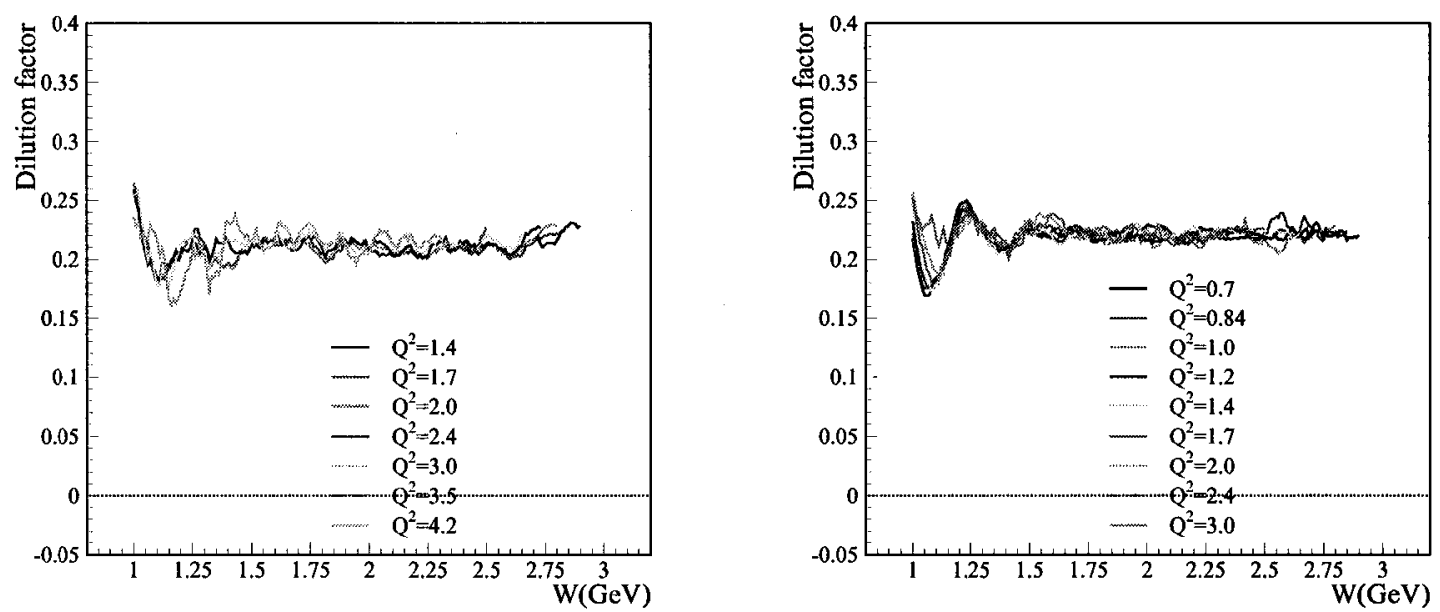

FIG. 83. The dilution factor plotted as a function of $W$ for the $5.6 \mathrm{GeV}$ data. The left plot is for inbending torus setting and the right plot is for outbending torus setting. 
As with the $1.6 \mathrm{GeV}$ data it shows minimal $Q^{2}$ dependence.

\subsubsection{Backgrounds}

The number of events recorded as electrons also contains a small percentage of events coming from charge symmetric processes as well as misidentified pions. These events provide an additional background and therefore have to be taken into account. In this section details about how these contaminations were determined and how they were applied to the measured asymmetry will be discussed.

\section{Pion contamination}

All the electron cuts were chosen carefully to reject most of the negatively charged pions and to accept electrons. However there is a small contamination of pions that pass the electron cuts and are misidentified as electrons. The measured asymmetry is therefore diluted due to these additional pions in the electron sample and has to be corrected appropriately.

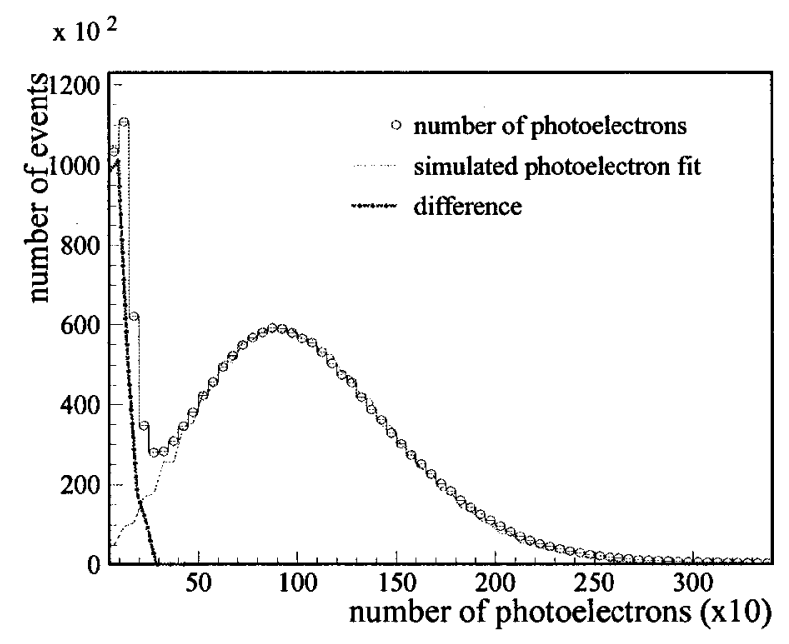

FIG. 84. The Cerenkov photoelectron spectrum was used to determine the pion contamination. The plot shows the photoelectron spectrum, the simulated photoelectrons which is fitted to the photoelectron spectrum and the difference between the two.

The pion contamination was determined by comparing the Cerenkov photoelectron spectrum after applying all the electron cuts other than the photoelectron cut with a photoelectron spectrum at the same kinematics simulated using a random 
number generator and the Cerenkov efficiency function (Fig. 84). The main assumption of this procedure is that the data photoelectron spectrum (after applying all the cuts other than the photoelectron cut) is due to photoelectrons produced by pions and electrons whereas the simulated photoelectron spectrum contains photoelectrons produced only by electrons. Therefore the difference between the two gives the spectrum of photoelectrons produced by pions. Then the photoelectron spectrum of a well defined pion sample was normalized to the difference spectrum. The pion contamination is the fraction of events attributable to pions above the photoelectron cut used for electron identification (Fig. 85).

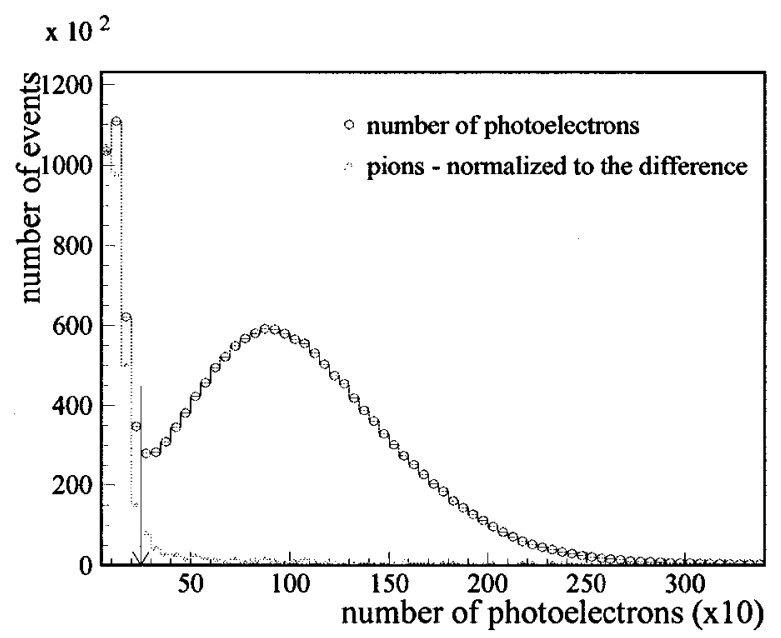

FIG. 85. The photoelectron spectrum and a well defined pion spectrum which has been normalized to the difference shown in Fig. 84. The arrow indicates the applied photoelectron cut.

Since pions with momenta above $2.7 \mathrm{GeV}$ produce Cerenkov radiation and cannot be separated from electrons, this method can be used only for momenta less than $2.7 \mathrm{GeV}$. Therefore to obtain a good estimate of pions at momenta greater than 3 $\mathrm{GeV}$, the total pion contamination for momenta less than $2.7 \mathrm{GeV}$ was fitted with an exponential function with two fit parameters $P_{1}$ and $P_{2}$,

$$
\text { total pion contamination }=P_{1} e^{-P_{2}(\text { momentum })}
$$

and was extrapolated to higher momenta (Fig. 86).

Similarly to obtain the pion contamination for momenta less than $2.7 \mathrm{GeV}$, the pion contamination (above the photoelectron cut) was fitted with a function similar to equation (166). In the intermediate region , $2.7 \mathrm{GeV}<$ momentum $<3.0 \mathrm{GeV}$, 


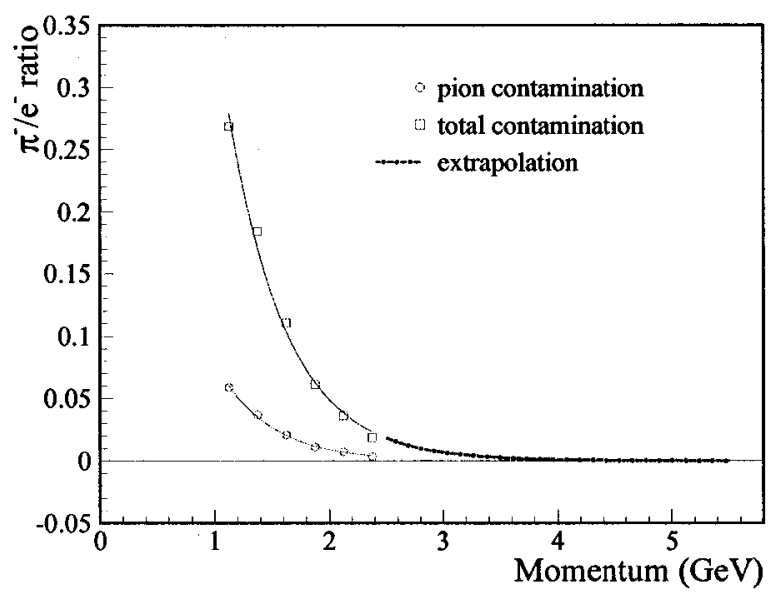

FIG. 86. Pion contamination vs momentum for one $\theta$ bin. The total number of pions to electrons ratio for number of photoelectrons greater than the applied photoelectron cut (pion contamination) and the total number of pions to total number of electrons (total contamination) as a function of momentum for $20<\theta<30$ are plotted. The two solid lines are the fit to the two ratios and the dashed line is the extrapolation of total contamination to higher momentums.

a linear combination of the two fitted functions was used. Fig. 87 shows the pion contamination, $R_{\pi^{-} / e^{-}}$, for different $Q^{2}$ bins as a function of the momentum.

\section{Pair symmetric correction}

Pair symmetric processes are electron positron pairs created in decay reactions. The electrons created in such processes are indistinguishable from electrons scattered from the target. One source of electron positron pair production is $\pi^{0} \rightarrow e^{+} e^{-} \gamma$ which occurs with a $1.2 \%$ probability. Additional pairs come from the reaction $\pi^{0} \rightarrow \gamma \gamma \rightarrow e^{+} e^{-} e^{+} e^{-}$. Bremsstrahlung photons can also produce $e^{+} e^{-}$pairs. However calculations done using formulas from Tsai show that pair production due to bremsstrahlung photons is negligible [57].

An estimation of electrons coming from pair production can be obtained by comparing the electron rates for a specific bin with positron rates for the same kinematic bin but taken with opposite torus setting, where the number of positrons detected in the region where inbending electrons were detected is assumed to be equal to the number of electrons created by pair symmetric processes. Choosing the opposite torus setting ensures that the detected positrons have the same acceptance as 


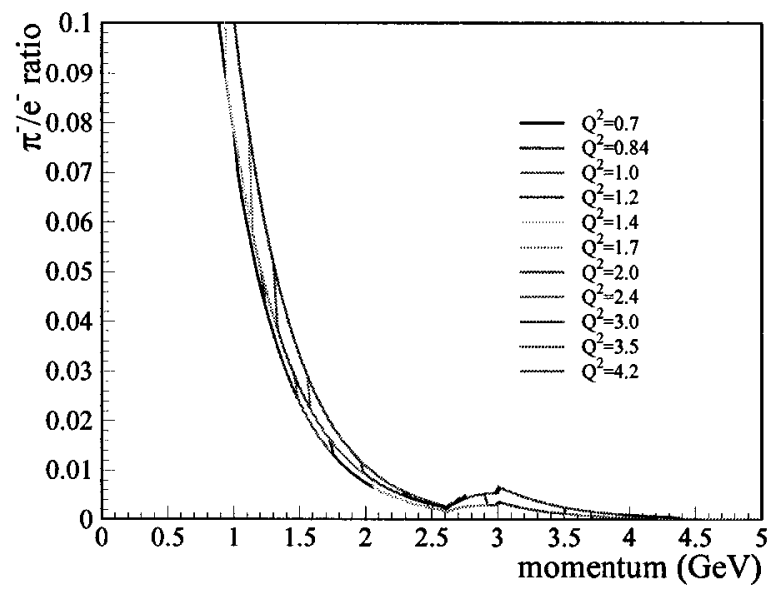

FIG. 87. Pion contamination as a function of momentum extracted using the method described in the text for different $Q^{2}$ values.

the electrons. To identify positrons, the same cuts that were used to identify electrons, except the charge, which is positive for positrons, were applied. To obtain the number of positrons as well as to subtract the misidentified $\pi^{+}$from the positrons, the same pion contamination procedure was applied. Then the number of photoelectrons due to electron and positron events were obtained and normalized to respective accumulated beam charge.

Another method to estimate the contamination was developed by Peter Bosted [58] in which the $E_{t o t} / P$ distribution discussed in section 5.5 for different kinematic bins for positrons and electrons are compared.

The estimate of the pair symmetric correction, $R_{e^{+}} / e^{-}$, obtained using these two methods for the inbending electron setting is compared in Fig. 88. It is evident that both methods give the same results. Therfore we used the $E_{\text {tot }} / P$ method to correct the pair symmetric background, since that method is more accurate for the outbending electron settings.

\section{Asymmetries due to background}

In addition to the corrections described above, the pions and positrons can also introduce some false asymmetries. To determine this asymmetries a well defined sample of pions and $e^{+}$was studied. The asymmetries due to pions $\left(A_{\pi^{-}} / e^{-}\right)$and electrons from the pair symmetric background $\left(A_{e^{+} / e^{-}}\right)$were measured using an identical analysis procedure as was used for the target electrons. The asymmetries so determined 

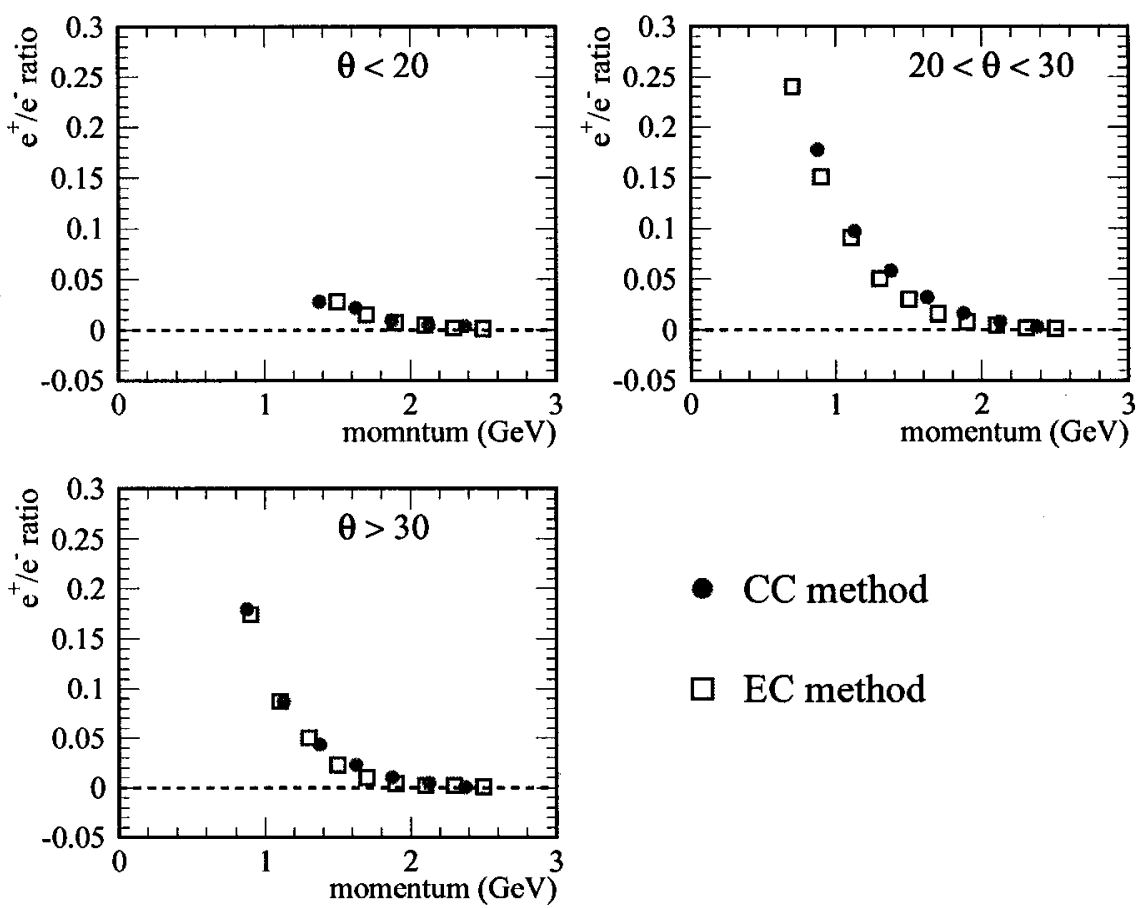

- CC method

EC method

FIG. 88. The ratio $e^{+} / e^{-}$extracted using two different methods as a function of momentum for three theta bins. The two methods give very similar results. The data are 5.x GeV inbending data.

are extremely small and are consistent with zero [58]. However, it was included in the systematic errors.

\section{Total correction due to background}

The final correction, $C_{b a c k}$, to the asymmetry given in equation (144) is then given by,

$$
C_{b a c k}=\frac{1-\left(R_{\pi^{-} / e^{-}} A_{\pi^{-} / e^{-}}\right)-\left(R_{e^{+} / e^{-}} A_{e^{+} / e^{-}}\right)}{1-R_{\pi^{-} / e^{-}}-R_{e^{+} / e^{-}}}
$$

where $A_{\pi^{-}} / e^{-}$and $A_{e^{+} / e^{-}}$were assumed to be zero.

\subsubsection{Electroweak Asymmetry}

Another source of false asymmetry is the asymmetry due to parity-violating effects, which arise due to the interactions between the electromagnetic and weak neutral currents. The asymmetry due to the interference of the $\gamma$ and $Z^{0}$ exchange amplitudes 
is given by,

$$
A^{E W}=\frac{\sigma_{R}-\sigma_{L}}{\sigma_{R}+\sigma_{L}}
$$

where $\sigma_{R}$ and $\sigma_{L}$ are the cross sections for the right and left handed electrons. The electroweak asymmetry is parameterized as [59],

$$
A^{E W} \approx-(1.0 \pm 0.3) \times 10^{-4} Q^{2} .
$$

The electroweak asymmetry is not sensitive to the direction of the target polarization. Therefore any false asymmetries due to this effect can be minimized by reversing the target polarization. During data taking half of the runs were taken with target polarization in one direction and the remaining half were taken with reversed target polarization. The correction is approximately $10^{-5}$ in the resonance region. Since the correction is very small the asymmetries were not corrected for this effect, which was considered negligible.

\subsubsection{Beam and Target Polarization}

To obtain asymmetries it is necessary to know the product of beam and target polarization. During data taking target polarization measurements made using the NMR system were used to monitor the polarization. The NMR coils on the EG1 target cells are located outside the cell and are more sensitive to the outer layer of the target material. However the entire target was not rastered during the data taking and the outer layer of the target was not always exposed to the beam. When the target is exposed to the beam the target material produces a local depolarization causing the inner region of the target to have a smaller polarization than the outer layer. Therefore the NMR readings reported erroneous values for beam polarization and often were larger than the real polarization of the target (Fig. 89). Hence the known asymmetry for elastic scattering off protons and neutrons was used to extract the product of beam and target polarization,

$$
P_{b} P_{t}=\frac{A_{q u a s i-e l}^{\text {meas }}}{F_{D F} A_{\text {quasi-el }}^{d}}
$$

where $A_{q u a s i-e l}^{\text {meas }}$ is the measured asymmetry in the quasi-elastic peak. The elastic asymmetry, $A_{e l}^{N}$, for the proton and neutron can be calculated using [60],

$$
A_{e l}^{N}=\frac{\cos \theta^{*} \sqrt{1-\epsilon^{2}} A_{1}+\sin \theta^{*} \sqrt{2 \epsilon(1-\epsilon)} A_{2}}{1+\epsilon \frac{Q^{2}}{\nu^{2}} \frac{G_{E}^{2}\left(Q^{2}\right)}{G_{M}^{2}\left(Q^{2}\right)}},
$$




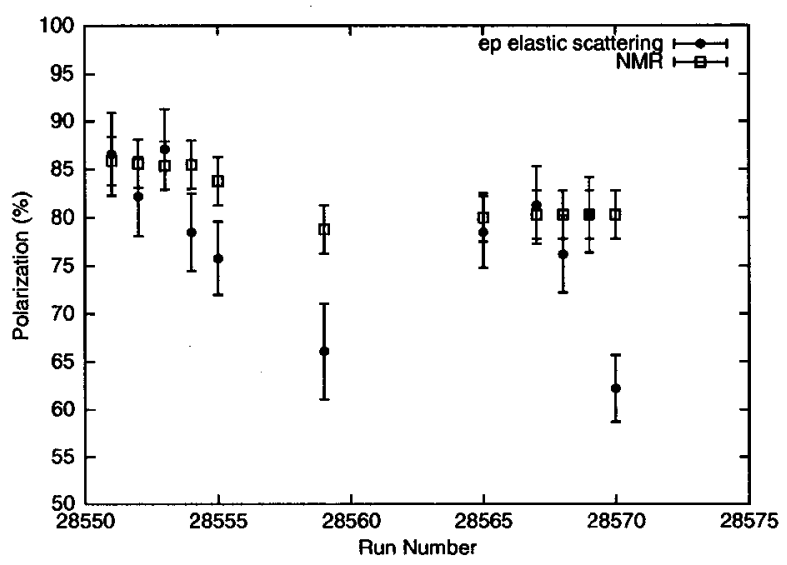

FIG. 89. Target polarization as a function of run number. An extensive study of the target polarization measured using the NMR and the target polarization extracted from the data has been done by the target group [46]. The plot shows the target polarization determined using these two methods for a few $\mathrm{NH}_{3}$ runs. It clearly shows large deviations for some runs, demonstrating the importance of extracting the polarization from ep elastic scattering data.

where $\theta^{*}$ is the polar angle between the target spin direction and the direction of the virtual photon and the virtual photon asymmetries $A_{1}$ and $A_{2}$ for elastic scattering are given by,

$$
\begin{aligned}
& A_{1}=1, \\
& A_{2}=\sqrt{\frac{Q^{2}}{\nu^{2}}}\left(\frac{G_{E}\left(Q^{2}\right)}{G_{M}\left(Q^{2}\right)}\right) .
\end{aligned}
$$

The quantities $G_{E}$ and $G_{M}$ are the electric and magnetic form factors of the nucleon, which can be calculated using [61],

$$
\begin{aligned}
G_{E}^{p}\left(Q^{2}\right) & =\frac{1.0087-0.1273 Q^{2}}{1+0.14 Q+3.01 Q^{2}+0.02 Q^{3}+1.20 Q^{4}+0.32 Q^{5}} \\
G_{M}^{p}\left(Q^{2}\right) & =\frac{\mu_{p}}{1+0.14 Q+3.01 Q^{2}+0.02 Q^{3}+1.20 Q^{4}+0.32 Q^{5}} \\
G_{M}^{n}\left(Q^{2}\right) & =\frac{\mu_{n}}{1-1.74 Q+9.29 Q^{2}-7.63 Q^{3}+4.63 Q^{4}} \\
G_{E}^{n}\left(Q^{2}\right) & =\frac{-a \mu_{n} \tau G^{\text {dipole }}\left(Q^{2}\right)}{1+b \tau}
\end{aligned}
$$

where $a=1.25, b=18.3$ and $G^{\text {dipole }}$ is,

$$
G^{\text {dipole }}=\left(1+\frac{Q^{2}}{0.71 \mathrm{GeV}^{2}}\right)^{-2}
$$


TABLE VII. Comparison of $A_{\text {quasi-el }}^{d}$ obtained using two different methods for the $5.735 \mathrm{GeV}$ outbending data. The PWIA numbers were obtained using a simulation [62] and $A_{e l}^{N}$ numbers were obtained using the elastic asymmetry for the proton and neutron.

\begin{tabular}{|l|c|c|}
\hline$Q^{2}\left(\mathrm{GeV}^{2}\right)$ & using $A_{e l}^{N}$ & PWIA \\
\hline 0.70 & 0.096 & 0.098 \\
0.84 & 0.112 & 0.113 \\
1.0 & 0.129 & 0.132 \\
1.2 & 0.151 & 0.154 \\
1.4 & 0.176 & 0.178 \\
1.7 & 0.207 & 0.208 \\
2.0 & 0.246 & 0.242 \\
2.4 & 0.290 & 0.286 \\
3.0 & 0.344 & 0.341 \\
\hline
\end{tabular}

The asymmetry, $A_{\text {quasi-el }}^{d}$ for the deuteron can be obtained by taking the weighted average (weighted by the corresponding elastic cross sections $\left(\sigma_{p(n)}^{e l}\right)$ elastic asymmetry given in equation (171) for the proton and neutron,

$$
A_{q u a s i-e l}^{d}=\frac{\sigma_{p}^{e l} A_{p}^{e l}+\sigma_{n}^{e l} A_{n}^{e l}}{\sigma_{p}^{e l}+\sigma_{n}^{e l}}
$$

and then correcting it for the D-state of the deuteron. We also used a simulation which calculates $A_{q u a s i-e l}^{d}$ in the Plane Wave Impulse Approximation (PWIA) using the most up-to-date fits to the nucleon form factors and a Paris potential wave function for the deuteron [62]. In the PWIA one assumes that the virtual photon is completely absorbed by one of the nucleons, which then leaves the nucleus without further interacting with the residual nucleus and thus can be treated as a plane wave. However, the numbers calculated using the two methods are very close and the difference in most cases is of order $10^{-3}$ (Table VII). Table VIII lists $A_{\text {quasi-el }}^{d}$ obtained using the simulation for the $1.6 \mathrm{GeV}$ and $5.6 \mathrm{GeV}$ beam settings integrated from $0.88 \mathrm{GeV}$ to $1 \mathrm{GeV}$ in $\mathrm{W}$ for different $Q^{2}$ values.

To obtain $A_{\text {quasi-el }}^{\text {meas }}$ for each $Q^{2}$ bin asymmetry corrected for dilution due to nontarget materials was integrated in $W$ from $0.88 \mathrm{GeV}$ to $1 \mathrm{GeV}$. Then $P_{b} P_{t}$ for each $Q^{2}$ bin was obtained by dividing it by the calculated asymmetry, $A_{q u a s i-e l}^{d}$. The final 
TABLE VIII. $A_{\text {quasi-el }}^{d}$ obtained [62] using a simulation for different $Q^{2}$ bins. The numbers contain the proper CLAS acceptance weighting. The inbending numbers are slightly different than the outbending numbers due to different acceptances.

\begin{tabular}{|l|c|c|c|}
\hline beam energy & $Q^{2}\left(\mathrm{GeV}^{2}\right)$ & $A_{\text {quasi-el }}^{d}$ (inbending) & $A_{\text {quasi-el }}^{d}$ (outbending) \\
\hline $1 . \mathrm{x} \mathrm{GeV}$ & 0.17 & 0.128 & \\
& 0.2 & 0.146 & \\
& 0.24 & 0.169 & \\
& 0.3 & 0.194 & \\
& 0.35 & 0.226 & \\
& 0.42 & 0.263 & \\
& 0.5 & 0.305 & 0.0981 \\
& 0.6 & 0.357 & 0.1136 \\
& 0.7 & & 0.1323 \\
& 0.84 & & 0.1541 \\
& 1.0 & & 0.1782 \\
& 1.2 & & 0.2083 \\
& 1.4 & 0.183 & 0.2424 \\
& 1.7 & 0.214 & 0.2869 \\
& 2.0 & 0.247 & 0.3418 \\
& 2.4 & 0.306 & \\
& 3.0 & 0.349 & \\
& 3.5 & 0.415 & \\
& 4.2 & 0.464 & \\
\hline
\end{tabular}


value of $P_{b} P_{t}$ for a particular data set was determined by doing a linear fit to all the $Q^{2}$ bins for that beam setting (Fig. 90).

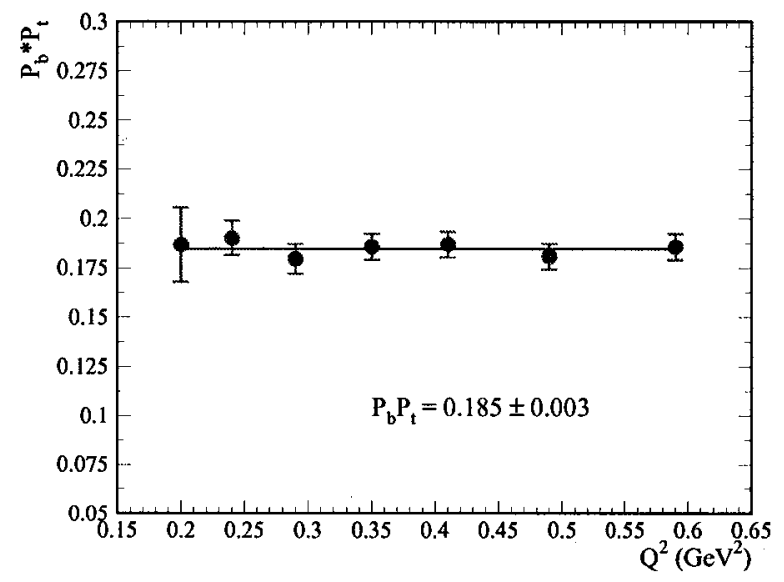

FIG. 90. The product of beam and target polarization was extracted by fitting the product extracted for each $Q^{2}$ bin with a constant function. The plot shows the fit for the $1.6 \mathrm{GeV}$ inbending data.

The product of beam and target polarization can also be extracted using elastic electron-proton coincidences. This method was used to extract $P_{b} P_{t}$ for the $5 . \mathrm{x} \mathrm{GeV}$ inbending data since it results in smaller errors. Electrons were selected using the electron cuts explained in the previous sections. The proton was defined by $0.7<$ $\beta<1.55$, number of photoelectrons $<0.5$ and a calorimeter cut of $E_{\text {total }} / P_{e l}<0.2$ and $E_{\text {inner }} / P_{e l}<0.08$. Further, to obtain elastic protons we required the total missing transverse momentum $<0.08 \mathrm{GeV}$, the missing energy $<0.15 \mathrm{GeV}$ and the missing momentum in the $\mathrm{z}$ direction $<0.12 \mathrm{GeV}$. To obtain the background, the same method used to obtain the inclusive scattering background was used. However, since we are looking only at elastic scattering from the proton in the deuteron all the formulas explained in section 5.7.2 were used with $b=0$. Fig. 91 shows a plot of the background subtraction for these elastic events. The calculated asymmetry for each $Q^{2}$ bin was obtained using equation (171) for the proton. However, an additional correction factor was applied to correct for the ratio of proton polarization to deuteron polarization due to the D-state,

$$
A_{e l}^{p}(\mathrm{D} \text { state corrected })=\left(1-1.5 \omega_{D}\right) A_{e l}^{p}
$$

To account for the acceptance of each $Q^{2}$ bin, the predicted value was calculated for each event and then an average was taken. The final $P_{b} P_{t}$ values were extracted 


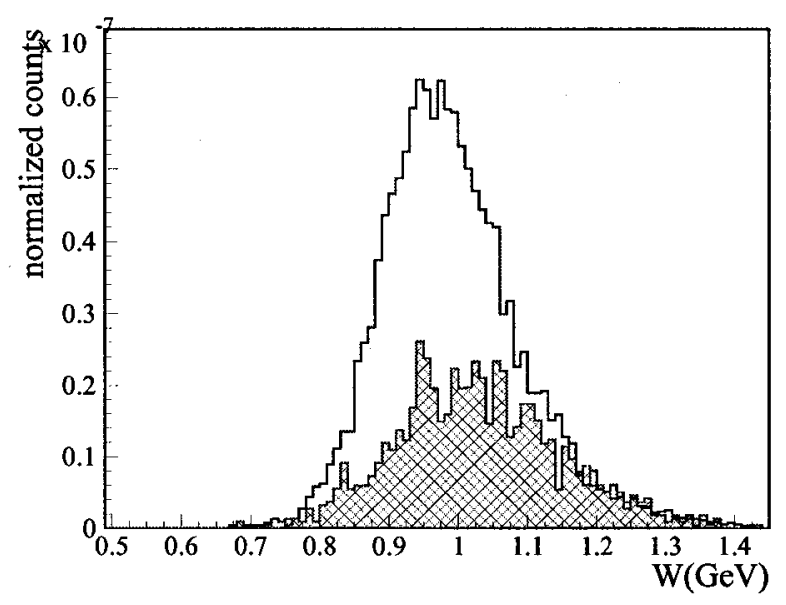

FIG. 91. $\mathrm{ND}_{3} W$ spectrum obtained using ep coincidences for $Q^{2}=2 \mathrm{GeV}^{2}$. The shaded region is the background.

by doing a fit to all $Q^{2}$ values. Fig. 92 shows the $P_{b} P_{t}$ values extracted for the $5.627 \mathrm{GeV}$ inbending data using this method and the inclusive method. The values obtained using ep coincidences gave smaller errors for $5 . \mathrm{x} \mathrm{GeV}$ inbending settings. Therefore the product obtained using this method was used in the analysis of $5 . x$ $\mathrm{GeV}$ inbending data.

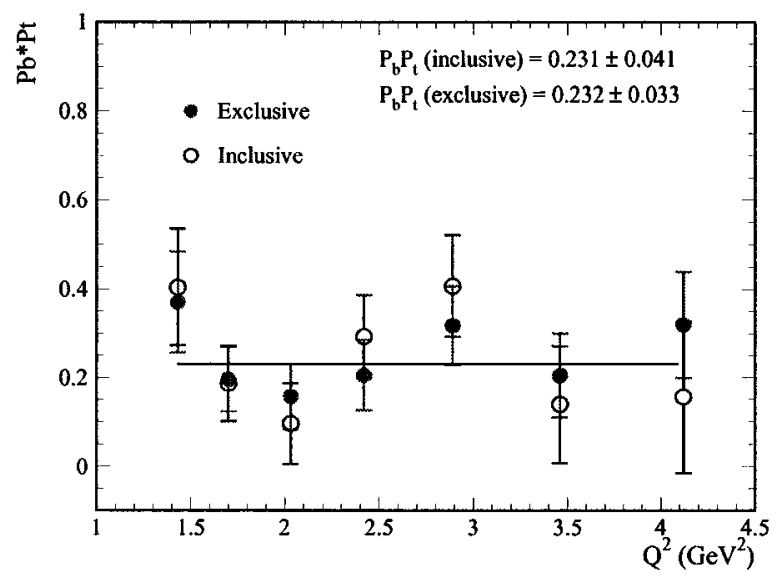

FIG. 92. Comparison of $P_{b} P_{t}$ values extracted using the two methods described in the text for the $5.627 \mathrm{GeV}$ inbending data.

Table IX lists the extracted $P_{b} P_{t}$ for all beam and target settings. All 5.x GeV inbending numbers were extracted using the exclusive method and all other numbers were extracted using the inclusive method. For each beam setting, the polarization for data taken with positive target polarization was compared with the data taken 
TABLE IX. $P_{b} P_{t}$ values extracted using data.

\begin{tabular}{|c|c|c|}
\hline beam energy $(\mathrm{GeV})$ & Torus setting & $P_{b} P_{t}$ \\
\hline 5.627 & Inbending & $0.230 \pm 0.033$ \\
\hline 5.735 & Inbending & $\begin{array}{l}\text { all target plus settings } 0.16 \pm 0.050 \\
\text { all target minus settings } 0.24 \pm 0.065\end{array}$ \\
\hline 5.735 & Outbending & $0.138 \pm 0.019$ \\
\hline 5.764 & Outbending & $0.115 \pm 0.018$ \\
\hline 1.606 & Inbending & $0.185 \pm 0.003$ \\
\hline 1.606 & Outbending & $0.179 \pm 0.009$ \\
\hline 1.707 & Outbending & $0.173 \pm 0.007$ \\
\hline
\end{tabular}

with negative target polarization. All beam settings gave numbers that were close except for the $5.735 \mathrm{GeV}$ inbending setting. Therefore, the $P_{b} P_{t}$ value extracted using all data combined (both target polarizations) for that beam setting was used in the analysis for all but the $5.735 \mathrm{GeV}$ inbending setting.

\subsubsection{Polarized Nitrogen and Residual Proton Correction}

In the analysis we assume that the only polarizable material in the target is the deuteron. However ${ }^{15} \mathrm{~N}$ nuclei as well as ${ }^{14} \mathrm{~N}$ nuclei in the target are also polarizable and contribute to the asymmetry [63]. In addition there is a small percentage of other residual nuclei such as $\mathrm{NH}_{3}$ and $\mathrm{ND}_{2} \mathrm{H}$ which introduce polarizable protons. For a target that contains all these materials, the measured count rates, $n^{+}$and $n^{-}$ for positive and negative helicity can be written as [63],

$$
n^{+}+n^{-}=\Phi\left(N_{D} \sigma_{D}+N_{P} \sigma_{P}+N_{15} \sigma_{15 N}+N_{14} \sigma_{14 N}+N_{\nu} \sigma_{\nu}\right),
$$

where,

$\Phi$ includes the acceptance and the flux of the incident beam,

$N_{D}$ is the number density of deuterons,

$N_{P}$ is the number density of protons,

$N_{14}$ is the number density of ${ }^{14} \mathrm{~N}$,

$N_{15}$ is the number density of ${ }^{15} \mathrm{~N}$, 
$N_{\nu}$ is the number density of unpolarized particles,

and $\sigma_{i}$ are the corresponding radiated cross sections. Similarly,

$$
\begin{aligned}
n^{+}-n^{-} & =P_{b} \Phi\left(N_{D} \sigma_{D} A_{D} P_{D}+N_{p} \sigma_{P} A_{P} P_{P}+N_{15} \sigma_{15 N} A_{15} P_{15}\right. \\
& \left.+N_{14} \sigma_{14 N} A_{14} P_{14}+N_{\nu} \sigma_{\nu} A_{\nu} P_{\nu}\right)
\end{aligned}
$$

where $A_{D}, A_{P}, A_{15}, A_{14}$ are the corresponding radiated asymmetries and $P_{D}, P_{P}$, $P_{15}, P_{14}$ are the corresponding polarizations of the materials given above. According to the nuclear shell model [63],

$$
\begin{aligned}
\sigma_{15 N} A_{15 N} & =-\frac{1}{3} g_{e m c}^{15 N} \sigma_{P} A_{P}, \\
\sigma_{14 N} A_{14 N} & =-\frac{1}{3} g_{e m c}^{14 N}\left(\sigma_{P} A_{P}+\sigma_{n} A_{n}\right) \approx-\frac{1}{3} g_{e m c}^{14 N} \frac{\sigma_{D} A_{D}}{\gamma},
\end{aligned}
$$

where $g_{e m c} \approx 1$ is a correction factor for the EMC effect [39], $\gamma=\left(1-\omega_{D}\right)$ corrects for the D-state probability of the deuteron and $\sigma_{n}$ and $A_{n}$ are the cross section and the asymmetry of the neutron. The term $-1 / 3$ arises due to the net alignment of the spin of the unpaired proton with the total orbital angular momentum. The unpaired proton in ${ }^{15} \mathrm{~N}$ is in a $j=1 / 2$ state with orbital angular momentum one. According to Clebsch-Gordon coefficients the probability of this unpaired proton aligning its spin with (against) the total angular momentum is $1 / 3(2 / 3)$, resulting in a net factor of $-1 / 3$. After simplifying equations (181) and (182) with the use of equations (183) and (184), and using the definition of the dilution factor,

$$
f_{D F}=\frac{N_{D} \sigma_{D}}{N_{D} \sigma_{D}+N_{P} \sigma_{P}+N_{15} \sigma_{15 N}+N_{14} \sigma_{14 N}+N_{\nu} \sigma_{\nu}}
$$

$A_{D}$, which is the asymmetry only due to the polarized deuteron, can be extracted,

$$
A_{D}=C_{1}\left(\frac{A_{\text {raw }}}{f_{D F} P_{b} P_{D}}-C_{2}\right) .
$$

With the approximations,

$$
\begin{gathered}
P_{15 N} \approx-P_{14 N} \equiv P_{N} \\
g_{e m c}^{14 N} \approx g_{e m c}^{15 N} \equiv g_{E M C}
\end{gathered}
$$

the factors $C_{1}$ and $C_{2}$ are given by [63],

$$
\begin{aligned}
& C_{1}=\frac{1}{1-\eta_{P}+D_{n} /\left(1-1.5 \omega_{D}\right)}, \\
& C_{2}=\frac{\sigma_{P}}{\sigma_{D}}\left(D_{n}-D_{p}\right) A_{P}
\end{aligned}
$$


where,

$$
\begin{aligned}
D_{n} & =\eta_{N} \frac{P_{N}}{P_{D}} \frac{g_{E M C}}{9}, \\
D_{p} & =\eta_{P} \frac{P_{P}}{P_{D}}-\left(1-\eta_{N}\right) \frac{P_{N}}{P_{D}} \frac{g_{E M C}}{9} \\
\eta_{P} & =\frac{N_{P}}{N_{D}+N_{P}} \approx 0.015, \\
\eta_{N} & =\frac{N_{14}}{N_{14}+N_{15}} \approx 0.02 .
\end{aligned}
$$

The ${ }^{15} \mathrm{~N}$ polarization is given by [63],

$$
P_{N}=-0.40 P_{D}
$$

The proton polarization can be written as,

$$
\begin{gathered}
P_{P}=0.191+0.683 P_{D} \text { for } \quad P_{D}>0.16 \\
=1.875 P_{D} \quad \text { for } \quad P_{D} \leq 0.16
\end{gathered}
$$

After substituting above numbers, $C_{2}$ is approximately given by [63],

$$
C_{2} \approx-0.03 A_{P}
$$

The factor $C_{1} \approx 1.02$ and is negligible. The factor $C_{2}$, which contains the proton asymmetry is non-negligible. However, the correction is approximately $3 \%$ of the asymmetry and was treated as a systematic error.

\subsubsection{Radiative Corrections}

Theoretical calculations of physics quantities such as $g_{1}, A_{1}$ and $\Gamma_{1}$ that are derived using $A_{\|}$assume that the scattering is due to one photon exchange (Born scattering). However, in practice there are other higher order physics processes beyond Born scattering that have to be taken into account. Therefore it is necessary to calculate corrections due to other processes and apply them to obtain the correct value of $A_{\|}$due to Born scattering. Since the electromagnetic coupling constant, $\alpha_{E M}$, is very small these corrections can be calculated using perturbative methods. In general radiative corrections can be divided into two main categories, internal radiative corrections, and external radiative corrections. Internal radiative corrections (Fig. 93) take into account the higher order QED processes that occur at the time 
(a)

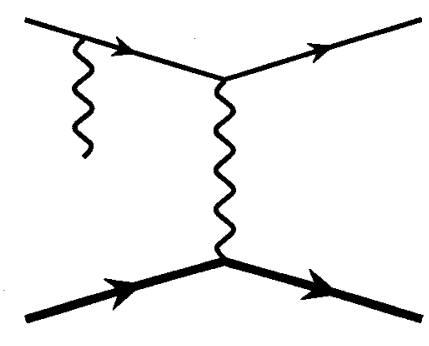

(c)

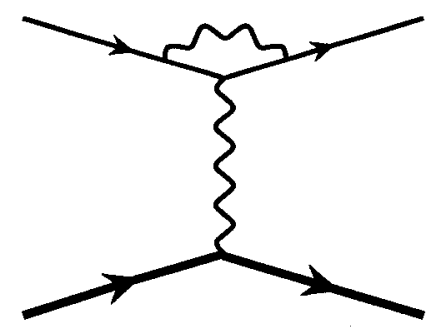

(b)

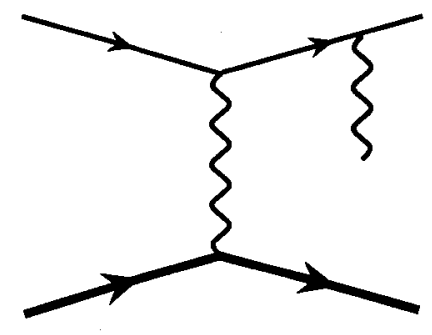

(d)

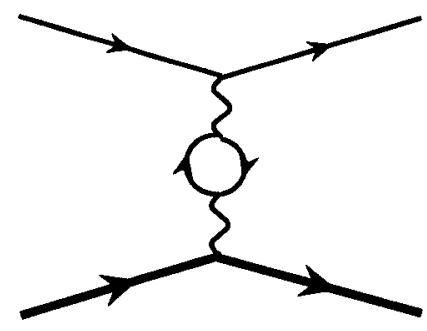

FIG. 93. The figure shows the Feynman diagrams for internal radiative corrections. (a) and (b) are internal bremsstrahlung, (c) vertex correction and (d) vacuum polarization.

of interaction, while the external corrections take into account energy losses due to bremsstrahlung or ionization in the external material before or after the interaction. If the Born asymmetry $A_{\|}$given in equation (16) is written in terms of polarized, $\sigma_{\text {Born }}^{p}$, and unpolarized, $\sigma_{B o r n}^{u}$, Born cross section,

$$
A_{\|}=\frac{\sigma_{\text {Born }}^{p}}{\sigma_{\text {Born }}^{u}},
$$

then the internally radiated asymmetry, $A_{\|}^{\text {int }}$, can be obtained by adding the higher order terms to the polarized and unpolarized Born cross section,

$$
A_{\|}^{i n t}=\frac{\sigma_{B o r n}^{p}\left(1+\delta_{v}\right)+\sigma_{e l}^{p}+\sigma_{q}^{p}+\sigma_{i n}^{p}}{\sigma_{B o r n}^{u}\left(1+\delta_{v}\right)+\sigma_{e l}^{u}+\sigma_{q}^{u}+\sigma_{i n}^{u}},
$$

where $\delta_{v}$ is the electron vertex correction and vacuum polarization corrections for lepton and hadrons, $\sigma_{e l}$ is the contribution of the radiative tail from the elastic peak, $\sigma_{q}$ is the contribution of the radiative tail from quasi elastic scattering and $\sigma_{i n}$ is the contribution of the radiative tail from the inelastic cross section. To obtain the fully radiated asymmetry, external radiative corrections also have to be taken into account. If we assume that the probability of an electron with initial energy $E_{0}$ to 
have final energy $E$ after traveling $t$ radiation lengths is given by $I_{b}\left(E_{0}, E, t\right)$, then the fully radiated cross section $\sigma_{\text {rad }}^{p(u)}$ can be obtained by [64],

$$
\begin{aligned}
& \sigma_{r a d}^{p}\left(E_{0}, E_{F}^{\prime}, \theta\right)= \\
& \int_{E_{\text {min }}}^{E_{0}} \int_{E_{F}^{\prime}}^{E_{\text {max }}^{\prime}} I_{b}\left(E_{0}, E, t_{i}\right) \sigma_{i n t}^{p}\left(E, E^{\prime}, \theta\right) I_{b}\left(E^{\prime}, E_{F}^{\prime}, t_{f}\right)\left[1-D\left(E_{0}, E^{\prime}, Z\right)\right] d E d E^{\prime}
\end{aligned}
$$

and

$$
\sigma_{r a d}^{u}\left(E_{0}, E_{F}^{\prime}, \theta\right)=\int_{E_{m i n}}^{E_{0}} \int_{E_{F}^{\prime}}^{E_{m a x}^{\prime}} I_{b}\left(E_{0}, E, t_{i}\right) \sigma_{i n t}^{u}\left(E, E^{\prime}, \theta\right) I_{b}\left(E^{\prime}, E_{F}^{\prime}, t_{f}\right) d E d E^{\prime},
$$

where $t_{i}$ and $t_{f}$ are the thicknesses of the material through which the electron travels before and after the interaction, $E_{F}^{\prime}$ is the final electron energy, $E_{\min }$ and $E_{\max }$ are the minimum and maximum energy defined by elastic scattering, $D\left(E_{0}, E^{\prime}, Z\right)$ is the electron depolarization correction [65] which corrects for the depolarization of the electron due to bremsstrahlung and $Z$ is the nuclear charge of the target.

Both internal and external corrections have been incorporated into a program called RCSLACPOL developed at SLAC by Linda Stuart, where the internal corrections are based on an approach by Kuchto and Shumeiko [66] and the external corrections by Tsai [64]. To obtain the radiated asymmetry the program uses the Born asymmetry as the input, calculated using a parameterization of polarized and unpolarized structure functions, and then applies all the radiative effects described above. The radiative correction to the measured asymmetry, $A_{\|}^{\text {meas }}$, consists of an additive $\left(A_{R C}\right)$ and a multiplicative $\left(F_{R C}\right)$ term [67],

$$
A_{\|}^{\text {Born }}\left(W, Q^{2}\right)=\frac{A_{\|}^{\text {meas }}\left(W, Q^{2}\right)}{F_{R C}\left(W, Q^{2}\right)}+A_{R C}\left(W, Q^{2}\right) .
$$

The factor $F_{R C}$ accounts for the fraction of the measured rate that does not come from the radiated elastic and quasi-elastic tails [59],

$$
F_{R C}=\frac{\sigma_{B o r n}^{u}\left(1+\delta_{v}\right)+\sigma_{i n}^{u}}{\sigma_{B o r n}^{u}\left(1+\delta_{v}\right)+\sigma_{e l}^{u}+\sigma_{q}^{u}+\sigma_{i n}^{u}},
$$

and is treated as a "dilution" of the contribution of $A_{\|}^{\text {Born }}\left(W, Q^{2}\right)$ to $A_{\|}^{\text {meas }}\left(W, Q^{2}\right)$. This term has particularly been included to properly propagate the statistical errors. The statistical error of a given kinematic bin is determined by the number of events recorded for that bin and is governed by the unpolarized radiated cross section while the true statistical error of the bin should be governed by the unpolarized Born cross 
section. The $F_{R C}$ term takes into account this additional dilution caused by radiated elastic and quasielastic tails and corrects the statistical errors of the measured assymetry, $\delta A_{\|}^{\text {meas }}$, in a proper manner,

$$
\delta A_{\|}^{\text {Born }}\left(W, Q^{2}\right)=\frac{\delta A_{\|}^{\text {meas }}\left(W, Q^{2}\right)}{F_{R C}\left(W, Q^{2}\right)} .
$$

The $A_{R C}$ term can then be viewed as the difference between the Born asymmetry and the "dilution corrected" radiated asymmetry. Fig. 94 shows the corrected and uncorrected asymmetry for one $Q^{2}$ bin.

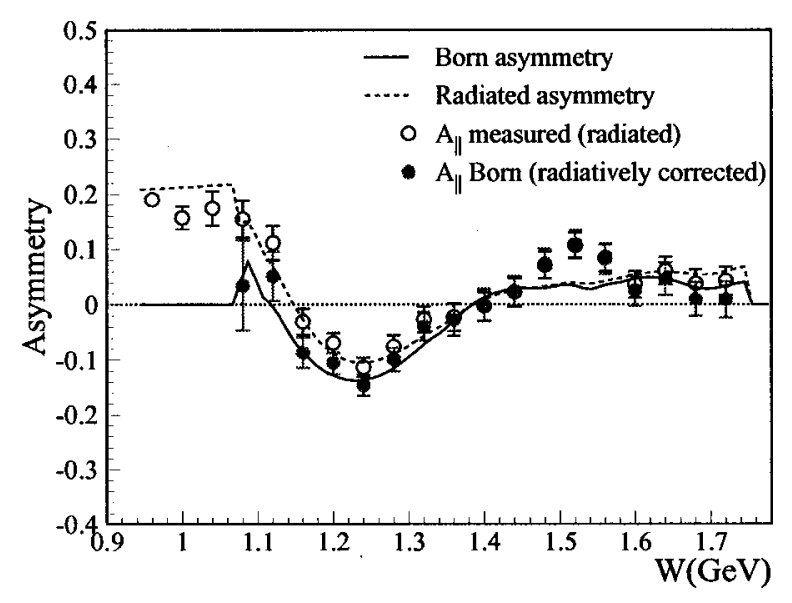

FIG. 94. The figure shows $A_{\|}^{\text {rad }}$ and $A_{\|}^{\text {Born }}$ as a function of $W$ for a typical $Q^{2}$ bin. The Born (solid line) and radiated (dashed line) asymmetries are calculated with our model.

\subsection{MODEL INPUT}

To extract the physics quantities of interest, $A_{1}$ and $g_{1}$, it is necessary to separate the virtual photon asymmetries $A_{1}$ and $A_{2}$ from the measured asymmetry $A_{\|}$. As has previously been mentioned (section 2.1.3) there are two ways of separating $A_{1}$ and $A_{2}$. However, since the 1.x and 5.x GeV data analyzed here did not have many overlapping $Q^{2}$ bins, it was not possible to do the "Rosenbluth-like" separation of $A_{1}$ and $A_{2}$. In addition a knowledge of quantities $R$ and $F_{1}$, which were not measured, is also required to obtain $A_{1}$ and $g_{1}$. In order to calculate the full integral, $\Gamma_{1}$, a knowledge of $g_{1}$ in the unmeasured region is necessary as well. As explained in the previous section a model of existing world data is also required to do radiative corrections. For all these purposes a model, which is a parameterization of existing world data 
collected by Linda Stuart, Thia Keppel, Keith Griffioen, Frank Wesselmann and Sebastian Kuhn, was developed [62]. In the following subsections details about how each of these quantities were modeled will be discussed. A more detailed discussion of the parameterization is given in [59]

\section{Virtual photon asymmetry $A_{1}$}

- In the DIS region a $Q^{2}$ and $x$ dependent fit to the world data was done [68]. The fit contains data in the $Q^{2}>1 \mathrm{GeV}^{2}$ and $W>2 \mathrm{GeV}$ region.

- In the resonance region a combination of DIS data extrapolated to the resonance region and the output from the code $\mathrm{AO}$ with non-resonant background turned off was used to obtain a reference model. The code $\mathrm{AO}$ uses a fit to exclusive electroproduction data to obtain the parameters for the resonant and non-resonant transition amplitudes. Then results from EG1a data were used to optimize the parameters.

- The fit was constrained to reach $A_{1}=1$ at pion threshold as well as by the value of the GDH sum rule at $Q^{2}=0$.

\section{Virtual photon asymmetry $A_{2}$}

- In the DIS region an iterative fit to the Wandzura-Wilczek relation, which depends only on $g_{1}$ ( see equation (56)) was performed.

- In the resonance region (up to $W=2 \mathrm{GeV}$ ) $A_{2}$ was calculated using the code MAID2000 [69], which is a unitary isobar model [70] of the resonance region developed at Mainz, based on fits to the existing electro and photo-production data. The nonresonant background is taken into account by using standard Born terms and vector meson exchange. A smooth transition for $A_{2}$ to the DIS region was required.

\section{Unpolarized structure functions $F_{1}$ and $F_{2}$}

- In the DIS region a fit, which is a fifteen parameter function, to $F_{2}$ that was done by the NMC collaboration was used [71]. The fit includes data obtained from inclusive muon scattering in the kinematic range $0.006<x<0.6$ and $Q^{2}$ between 0.5 to $75 \mathrm{GeV}^{2}$ together with the existing world data, which all 
together covers the region from 0.006 to 0.9 in $x$. The total error on the data used in the fit is between $1.5 \%$ and $5 \%$.

- In the resonance region a fit to world data, which includes recent unpolarized structure function results from Jlab, performed by Thia Keppel was used [62]. The model, which is a twenty four parameter fit, includes three resonances and three background terms. All fits were modified to connect smoothly to the real photon data as $Q^{2} \rightarrow 0$.

\section{The ratio $R$}

- The ratio $R$ was calculated using the SLAC fit "R1998" [72], which is an update to the SLAC/Whitlow fit, "R1990" [12]. The data used in the fit cover the kinematic range of $0.005 \leq x \leq 0.86$ and $0.5 \leq Q^{2} \leq 130 \mathrm{GeV}^{2}$. The model includes three different parameterizations for $R$. In the analysis the average of the three parameterizations was taken. The uncertainty in $\mathrm{R}$ due to statistical fluctuations of the data is given by the error of the fit,

$$
\delta R\left(x, Q^{2}\right)=0.0078-0.013 x+\frac{0.070-0.39 x+0.70 x^{2}}{1.7+Q^{2}} .
$$

The fit has a confidence level of $73 \%$ for all the data used in the fit.

- The low $Q^{2}$ region was modeled by either assuming that $R$ is constant or that $R \rightarrow 0$ as $Q^{2} \rightarrow 0$.

In all these cases the resonance region and the DIS region were combined using a smooth interpolation.

\subsection{COMBINING DIFFERENT GROUPS OF RUNS}

The final step in the analysis is to combine different groups of runs which includes runs with different target polarization, slightly different beam energies and outbending vs. inbending torus setting. In this analysis $A_{1}$ and $g_{1}$ were calculated for each setting and kinematic bin separately and were combined at the end. The average over different groups were performed in the following sequence.

- All target plus settings of $5.735 \mathrm{GeV}$ inbending data were combined with the all target plus settings of the $5.627 \mathrm{GeV}$ inbending data. 
- All target minus settings of $5.735 \mathrm{GeV}$ inbending data were combined with the all target minus settings of the $5.627 \mathrm{GeV}$ inbending data.

- All target plus settings of 5.x GeV inbending data were combined with the all target minus settings of the $5 . \mathrm{x} \mathrm{GeV}$ inbending data.

- $5.735 \mathrm{GeV}$ outbending data were combined with the 5.764 outbending data.

- 5.x GeV inbending data were combined with the 5.x GeV outbending data.

- $1.606 \mathrm{GeV}$ outbending data were combined with the $1.707 \mathrm{GeV}$ outbending data.

- 1.x GeV outbending data were combined with the $1.606 \mathrm{GeV}$ inbending data.

The quantities $g_{1}$ and $A_{1}(f)$ for each bin $(i)$ from different run groups $(j)$ were combined using the weighted mean with the final statistical errors $\left(\sigma_{i}\right)$,

$$
\begin{aligned}
f_{i}(\text { average }) & =\frac{\sum_{j} f_{i j} / \sigma_{i j}^{2}}{\sum_{j} 1 / \sigma_{i j}^{2}}, \\
\sigma\left(f_{i}(\text { average })\right) & =\frac{1}{\sqrt{\sum_{j} 1 / \sigma_{i j}^{2}}} .
\end{aligned}
$$

Before combining, each of these data sets were compared through a z-test,

$$
z_{i}=\frac{f_{i}(j)-f_{i}\left(j^{\prime}\right)}{\sqrt{\left.\sigma_{i}^{2}(j)\right)+\sigma_{i}^{2}\left(j^{\prime}\right)}}
$$

for each of the overlapping kinematic bin. The $\chi^{2}$ value and the average z-score,

$$
z_{\text {average }}=\frac{\sum_{i} z_{i}}{n}
$$

for all the $W$ bins for a given $Q^{2}$ bin was then calculated to check the compatibility of each $Q^{2}$ bin that was combined. Fig. 95 shows the z-score values of the $5 . x \mathrm{GeV}$ inbending data taken with positive target polarization and negative target polarization for all $Q^{2}$ bins.

Table $\mathrm{X}$ and XI lists all the average $\mathrm{z}$ score values of each overlapping $Q^{2}$ bin for all the different data sets listed above. 
TABLE X. z score values of all the $5 . x \mathrm{GeV}$ data.

\begin{tabular}{|l|c|c|c|c|}
\hline Data sets & $Q^{2}\left(\mathrm{GeV}^{2}\right)$ & $z_{\text {average }}$ & number of bins & $\chi^{2}$ \\
\hline 5.735 target plus and & 1.43 & 0.089 & 162 & 1.067 \\
5.627 target plus & 1.70 & -0.060 & 152 & 1.136 \\
(Inbending) & 2.03 & -0.041 & 152 & 1.126 \\
& 2.42 & -0.016 & 148 & 0.928 \\
& 2.89 & -0.074 & 140 & 1.071 \\
& 3.46 & 0.071 & 127 & 0.855 \\
& 4.12 & 0.055 & 105 & 1.101 \\
\hline 5.735 target minus and & 1.43 & -0.141 & 162 & 1.010 \\
5.627 target minus & 1.70 & -0.044 & 152 & 1.035 \\
(Inbending) & 2.03 & 0.027 & 152 & 1.045 \\
& 2.42 & -0.111 & 148 & 0.982 \\
& 2.89 & 0.045 & 140 & 1.054 \\
& 3.46 & 0.054 & 127 & 1.027 \\
& 4.12 & -0.000 & 105 & 1.108 \\
\hline $5 . x$ target plus and & 1.43 & -0.067 & 162 & 1.003 \\
$5 . x$ target minus & 1.70 & -0.046 & 152 & 1.177 \\
(Inbending) & 2.03 & -0.036 & 152 & 1.058 \\
& 2.42 & 0.002 & 148 & 0.857 \\
& 2.89 & 0.019 & 140 & 0.979 \\
& 3.46 & 0.018 & 127 & 0.961 \\
& 4.12 & -0.016 & 105 & 0.972 \\
\hline 5.735 outbending and & 0.707 & -0.181 & 172 & 0.935 \\
5.764 outbending & 0.844 & -0.099 & 172 & 0.922 \\
& 1.01 & -0.009 & 162 & 1.281 \\
& 1.20 & -0.053 & 162 & 1.130 \\
& 1.44 & -0.037 & 162 & 1.116 \\
& 1.71 & -0.169 & 152 & 0.976 \\
& 2.05 & 0.015 & 152 & 0.765 \\
& 2.44 & 0.039 & 148 & 1.022 \\
& 2.92 & 0.093 & 140 & 0.995 \\
\hline $5 . x$ inbending & 1.44 & 0.105 & 162 & 0.903 \\
& 1.71 & 0.072 & 152 & 1.155 \\
& 2.05 & 0.174 & 152 & 1.094 \\
& 2.44 & 0.038 & 148 & 1.238 \\
& 2.92 & 0.180 & 140 & 1.032 \\
\hline & & & & \\
& & & & \\
& & &
\end{tabular}


TABLE XI. $\mathrm{z}$ score values of all the 1.x GeV data.

\begin{tabular}{|l|c|c|c|c|}
\hline Data sets & $Q^{2}\left(\mathrm{GeV}^{2}\right)$ & $z_{\text {average }}$ & number of bins & $\chi^{2}$ \\
\hline 1.607 outbending and & 0.0496 & 0.049 & 51 & 0.825 \\
1.724 outbending & 0.0592 & 0.110 & 51 & 1.018 \\
& 0.0707 & -0.015 & 51 & 1.103 \\
& 0.0844 & 0.016 & 51 & 0.991 \\
& 0.1010 & 0.072 & 51 & 1.206 \\
& 0.1200 & 0.211 & 51 & 0.978 \\
& 0.1440 & 0.141 & 51 & 1.230 \\
& 0.1710 & 0.099 & 51 & 0.869 \\
& 0.2050 & -0.130 & 49 & 0.608 \\
& 0.2440 & 0.091 & 49 & 1.254 \\
& 0.2920 & -0.045 & 49 & 1.028 \\
& 0.3480 & -0.109 & 46 & 0.972 \\
1.607 inbending & 0.4160 & -0.176 & 40 & 1.225 \\
\hline & 0.2050 & -0.032 & 49 & 0.810 \\
& 0.2440 & -0.135 & 49 & 1.019 \\
& 0.2920 & -0.032 & 49 & 0.847 \\
& 0.3480 & 0.243 & 46 & 1.155 \\
& 0.4160 & 0.167 & 40 & 1.050 \\
\hline
\end{tabular}



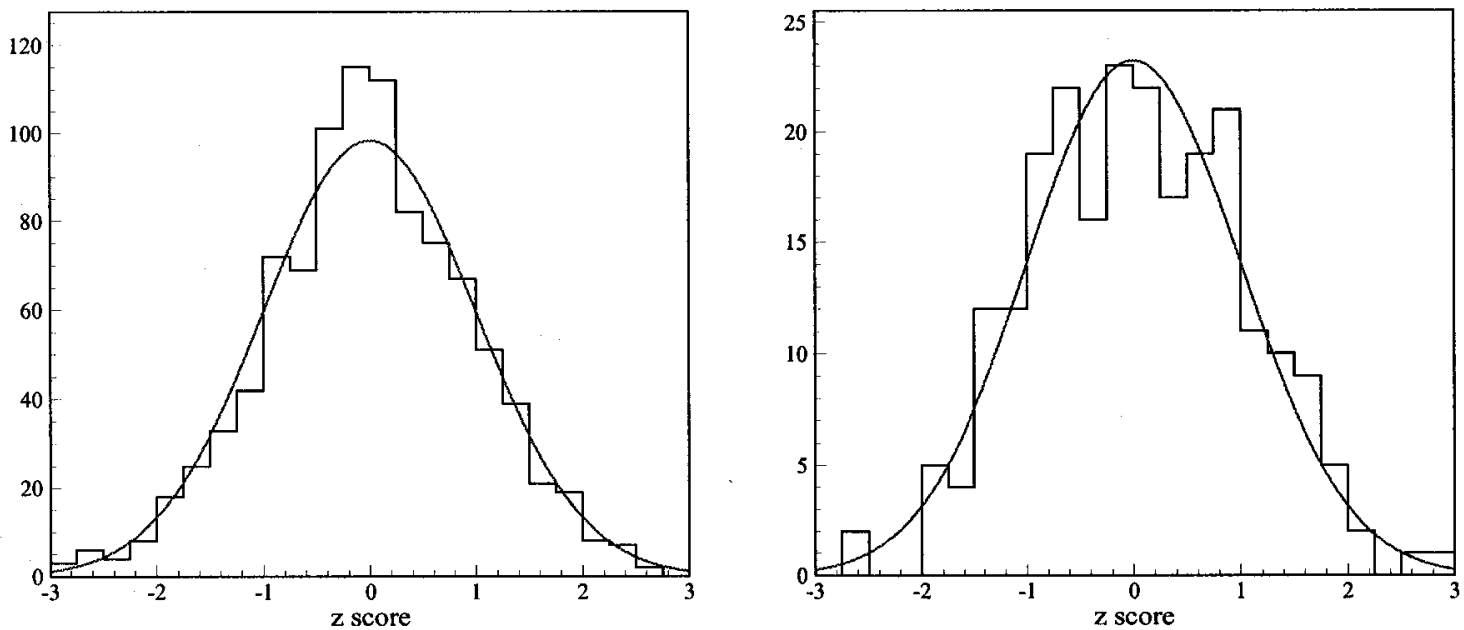

FIG. 95. Plot shows the z-score values for different data sets. The left plot shows the z-score values of all target plus settings of $5 . x \mathrm{GeV}$ inbending data and all target minus settings of the $5 . \mathrm{x} \mathrm{GeV}$ inbending data. The right plot shows the z-score values of inbending settings of the $1.607 \mathrm{GeV}$ data and outbending settings of the $1 . \mathrm{x} \mathrm{GeV}$ data. The red line is a Gaussian function with sigma equal to one.

\subsection{SYSTEMATIC ERRORS}

There are two main sources of systematic errors associated with the results of this analysis. The first source arises due to uncertainties in measurements. The second source arises due to uncertainties in the models that were used to extract the final physics results.

The first step in determining the systematic errors is to change a parameter or a parameterization, which is a source of systematic error, to another reasonable value and then redo the analysis and obtain new results. Then the systematic uncertainty due to that quantity is given by the difference between the results obtained using this new value and the results obtained using the standard value. For example, if the final value of $g_{1}$ for a given $\left(Q^{2}, x\right)$ bin, after changing a parameter $i$, is given by $g_{1(i)}$ and the standard value of $g_{1}$ is $g_{1(s)}$, then the systematic error in $g_{1}$ due to the uncertainty $i, \delta g_{1(i)}$, is calculated by,

$$
\delta g_{1(i)}\left(Q^{2}, x\right)=\left|g_{1(s)}\left(Q^{2}, x\right)-g_{1(i)}\left(Q^{2}, x\right)\right|
$$

The total systematic error, $g_{1(t o t)}\left(Q^{2}, x\right)$, was calculated by adding all the systematic 
errors in quadrature.

$$
\delta g_{1(t o t)}\left(Q^{2}, x\right)=\sqrt{\sum_{i} \delta g_{1(i)}^{2}\left(Q^{2}, x\right)}
$$

However, there were some exceptions as explained below. This section describes how each of these systematic uncertainties were determined.

\subsubsection{Dilution Factor}

The calculation of the dilution factor was modeled using ${ }^{12} \mathrm{C}$. Any difference between the simulated ${ }^{15} \mathrm{~N}$ spectrum and the real ${ }^{15} \mathrm{~N}$ background would give incorrect results. To minimize those uncertainties the ${ }^{15} \mathrm{~N}$ spectrum was simulated by fitting ${ }^{12} \mathrm{C}$ data to data taken with a ${ }^{15} \mathrm{~N}$ target as explained in section 5.7.2. However, the extraction of the parameters $a$ and $b$ depends on the measured length of ${ }^{15} \mathrm{~N}$ and the cross sections $\sigma_{n} / \sigma_{D}$, which were calculated using a model. The calculation of the dilution factor also depends on the physical length of the target. To estimate all these uncertainties the following steps were taken:

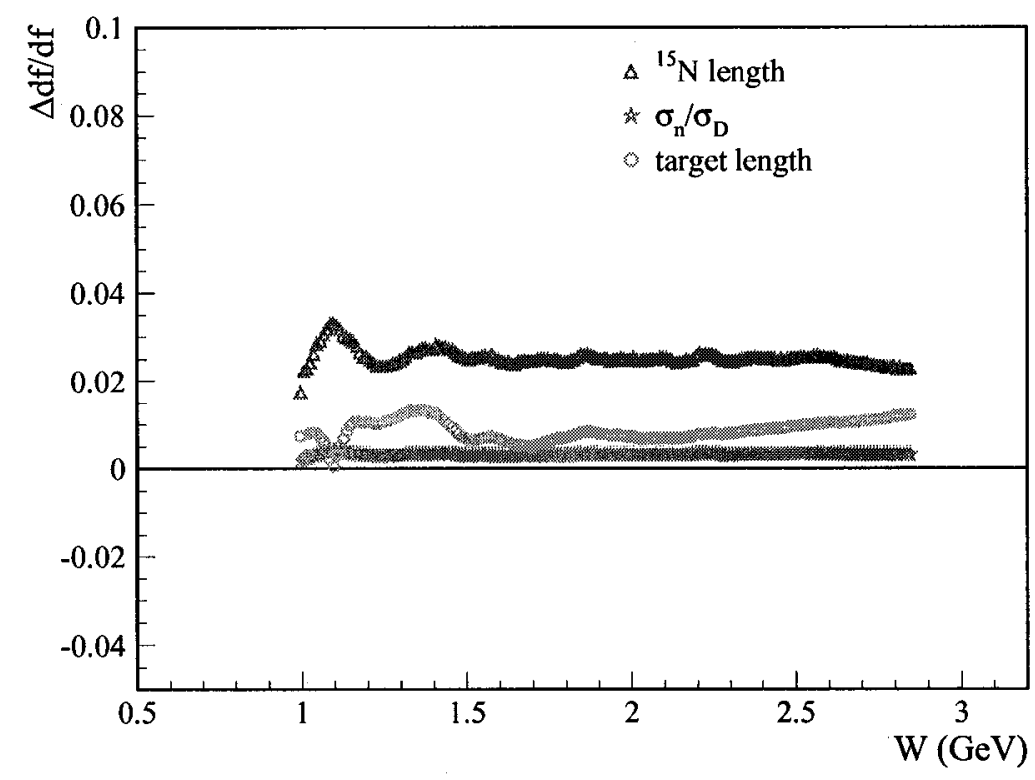

FIG. 96. The effect on the dilution factor due to different sources of systematic errors. The $\mathrm{Y}$ axis gives the change in dilution factor divided by the dilution factor that was used in the analysis.

- Changed the length of ${ }^{15} \mathrm{~N}$ from $0.5 \mathrm{~cm}$ to $0.52 \mathrm{~cm}$. The extracted value for $a$ and $b$ depend on the length of the ${ }^{15} \mathrm{~N}$ target. A new fit was performed with 
a target length of $0.52 \mathrm{~cm}$ and new values for $a$ and $b$ were extracted. This changed the dilution factor by approximately $\sim 3 \%$ (Fig. 96).

- In the inelastic region $\sigma_{n} / \sigma_{D}$ was calculated by dividing $F_{1}^{n} / F_{1}^{d}$, which was calculated using the model described in section 5.8. In the elastic region the elastic neutron cross sections were directly divided by the sum of proton and neutron cross sections, calculated using the Bosted parameterization [61] of form factors. Fig. 97 shows $\sigma_{n} / \sigma_{D}\left(Q^{2}, W\right)$ for a few $Q^{2}$ bins used in the analysis. The systematic uncertainty in the calculation was estimated by changing $\sigma_{n} / \sigma_{D}$ to 0.5 . Since this was also used in the determination of parameters $a$ and $b$, a new fit was performed and new $a$ and $b$ values were extracted. This changed the dilution factor by less than $1 \%$ (Fig. 96).

- In the analysis, the measured value of the physical length of the target was used in calculating the dilution factor. This can also be extracted from data as explained in section 5.7.2, which is very close to the measured value. However, to determine the systematic uncertainty on the measurement it was changed from $1.9 \mathrm{~cm}$ to $1.825 \mathrm{~cm}$. Fig. 96 shows the effect on the dilution factor which is about $1 \%$.

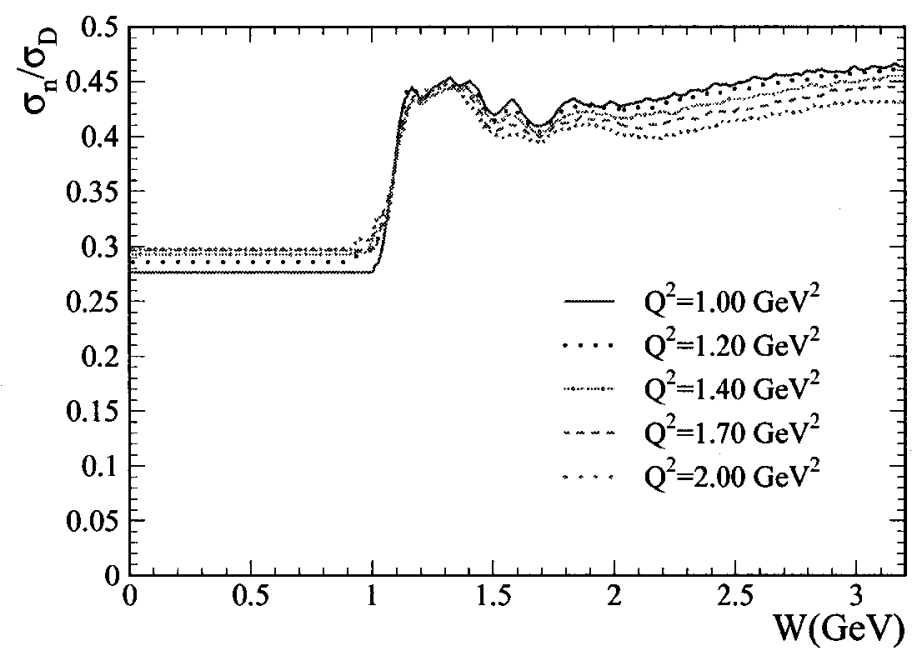

FIG. 97. The $\sigma_{n} / \sigma_{D}$ values that were used in the analysis. The numbers in the inelastic region were obtained using the model. More details are in the text. 


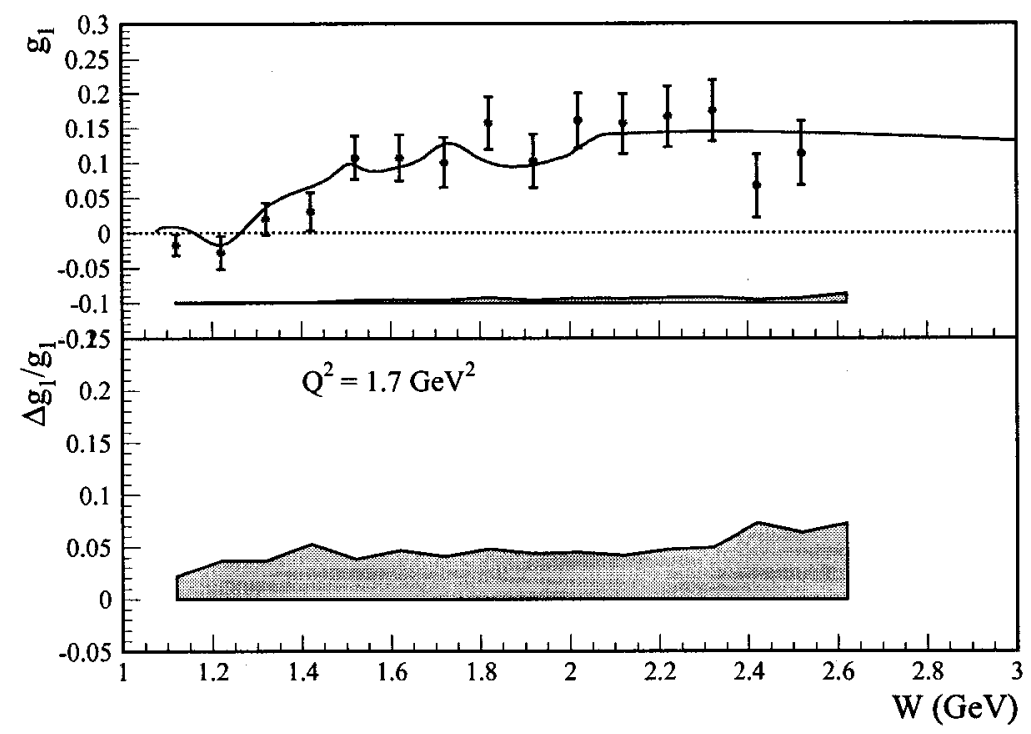

FIG. 98. The systematic error on $g_{1}$ due to the dilution factor compared to the statistical uncertainty for one $Q^{2}$ bin. The systematic error is shown as the the gray band on the top plot. The same quantity given as a percentage of the measured value of $g_{1}$ is shown in the bottom plot. The total systematic error on $g_{1}$ is approximately $5 \%$. The calculation of $g_{1}$ is discussed in the next chapter.

In each of the above cases the complete analysis was performed using the new parameters. The difference between the new results and the standard results was taken as the systematic uncertainty of that particular source. Fig. 98 shows the total systematic error due to the above mentioned sources on $g_{1}$ for one $Q^{2}$ bin.

\subsubsection{Background Processes}

Since the pair symmetric correction and pion contamination are small, the systematic errors due to those sources were determined by completely eliminating them. The systematic error is less than $1 \%$.

\subsubsection{Polarized Nitrogen and Proton Correction}

As explained in section 5.7.6 the correction due to nitrogen and the residual protons contain two terms. The factor $C_{1}$ is approximately equal to one and is therefore neglected. The systematic error was calculated by applying the term $C_{2}$ to the measured asymmetry. The factor $C_{2}$ contains the proton asymmetry. It was calculated using the radiated cross sections for the proton, which were obtained using the method 
described in section 5.7.7. The total systematic error on $g_{1}$ is approximately $3 \%$.

\subsubsection{Beam and Target Polarization}

The main source of error that contributes to the determination of beam and target polarization is of a statistical nature. As described in section 5.7.5 the product of beam and target polarization was extracted using data. However the error was not propagated as a statistical error. Instead one sigma of the error was added to the polarization used in the analysis of data, for one data set at a time and the whole analysis was performed keeping the polarization of the other data sets at the same value as was used in the analysis. The same procedure was performed for each individual data set given in Table II. The difference between the new result and the standard result for each data set is added in quadrature to obtain the final systematic error on the product of beam and target polarization. The average systematic uncertainty in the product of beam and target polarization is about $9 \%$ for the $5 . x$ $\mathrm{GeV}$ data and is much smaller for the $1 . \mathrm{x} \mathrm{GeV}$ data.

\subsubsection{Systematic Errors due to Models}

The extraction of asymmetries and the spin structure function $g_{1}$ as well as the calculation of the integral of $g_{1}$ in the unmeasured region depends on how we model some of the unmeasured parts that contributes to the final result. Therefore to determine systematic errors due to our model, different parameterizations were used [62].

\section{Asymmetries $A_{1}$ and $A_{2}$}

To determine uncertainties due to the parameterization of $A_{1}$ and $A_{2}$, the alternative parameterizations explained below were used (Fig. 99):

1. $A_{2}$ in the resonance and the DIS region were set to zero.

2. The parameters that calculate the standard resonance contribution to $A_{1}$ and $A_{2}$ were slightly changed. The standard value was still used in the DIS region. However, since our standard DIS calculation involves an integral over $g_{1}(y) / y$ from $y=x$ to $y=1$, it depends somewhat on the behavior of $A_{2}$ (and thus $g_{1}$ ) in the resonance region. Therefore this estimate of the systematic uncertainty is applicable in the DIS region as well as in the resonance region. 


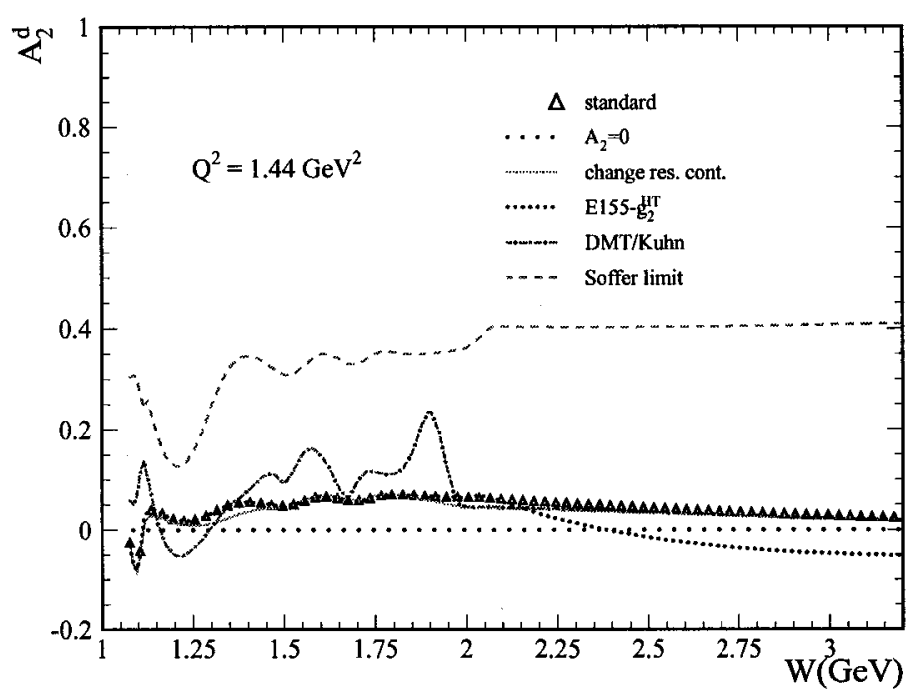

FIG. 99. Different parameterizations of $A_{2}$ used to calculate systematic errors. More details are in the text.

3. $A_{2}$ was modeled by taking into account the twist- 3 part, $g_{2}^{H T}$ given in equation (55), in addition to the $g_{2}^{W W}$ term. The term $g_{2}^{H T}$ is the deviation between the measured value of $g_{2}$ and the $g_{2}^{W W}$ term. This has been extracted from measured results and parameterized by the E155 collaboration [24] and was used to obtain $A_{2}$.

4. We used a new model (DMT/Kuhn) [62] for $A_{2}$ in the resonance region, which uses the DMT (Dubna-Mainz-Taipei) dynamical model for pion production [73]. The model also includes eta and kaon production. The resonance region was connected to higher $W$ values using a smooth interpolation to $g_{2}^{W W}$. An additional contribution was added in the resonance region to ensure that the Burkhardt-Cottingham sum rule is fulfilled.

5. Replace $A_{2}$ with the upper Soffer bound [74], $A_{2} \leq \sqrt{\left(1+A_{1}\right) / 2 R}$ in both the resonance and the DIS region. To calculate $R$, "R1998" was used. Since the Soffer limit (Fig. 99) gives an unreasonably large systematic error in the DIS region, where there are plenty of data available and it is well understood (Fig. 100), the Soffer limit was divided by $W$ to get a reasonable systematic error. 


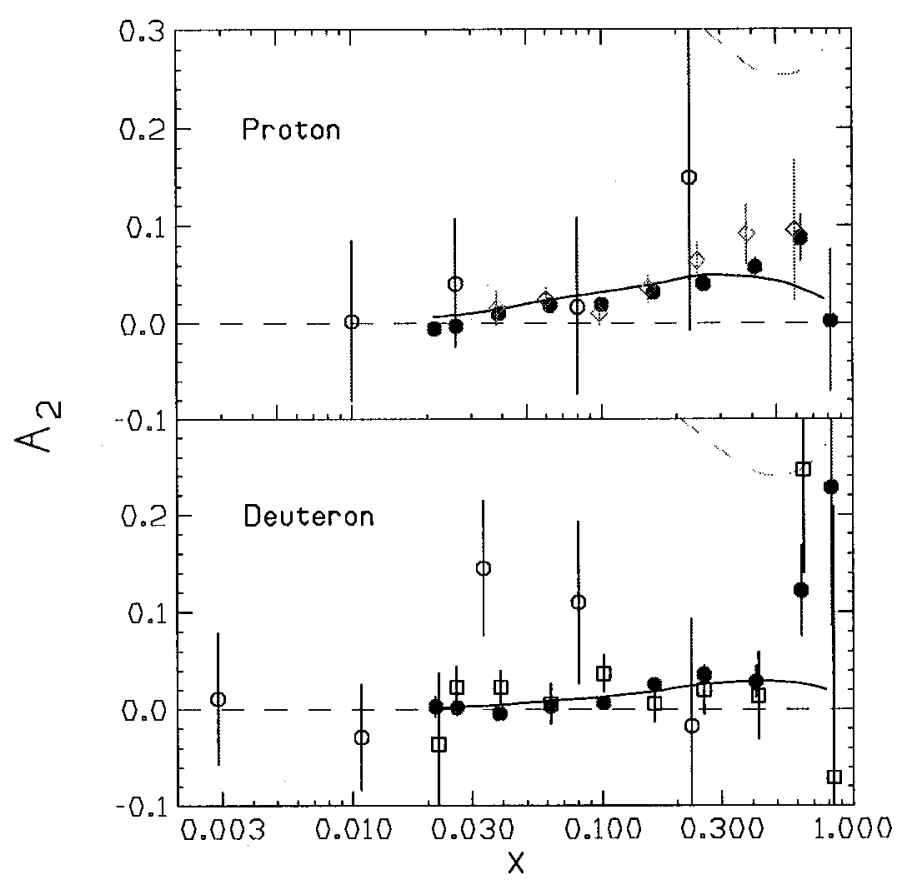

FIG. 100. Measurement of $A_{2}$ for the deuteron and proton [24]. The data are from E155 (solid circle and open square), E143 (open diamond) experiments at SLAC and EMC (open circles) results. The solid line is $A_{2}$ calculated using $g_{2}^{W W}$. The Soffer bound is given as a dashed line (upper right).

The overall systematic error was calculated by adding all the differences in quadrature and then dividing it by three,

$$
\delta g_{1\left(A_{1}, A_{2}\right)}\left(Q^{2}, x\right)=\sqrt{\frac{1}{3} \sum_{1}^{5}\left(g_{1}(\text { variable })-g_{1}(\text { standard })\right)^{2}}
$$

The total was divided by three because in some of the cases both $A_{1}$ and $A_{2}$ were varied. Fig. 101 shows the total systematic error due to the different asymmetry choices for one $Q^{2}$ bin. The systematic uncertainty is about 10\%. Fig. 102 shows the contribution to the total systematic error from the above mentioned models.

\section{Structure functions $F_{1}, F_{2}$ and $R$}

To estimate the systematic uncertainties due to our model of the structure functions, the following alternatives were used (Fig. 103 and Fig. 104):

1. A new value of $R$ was obtained by taking into account three sources of uncertainty: 


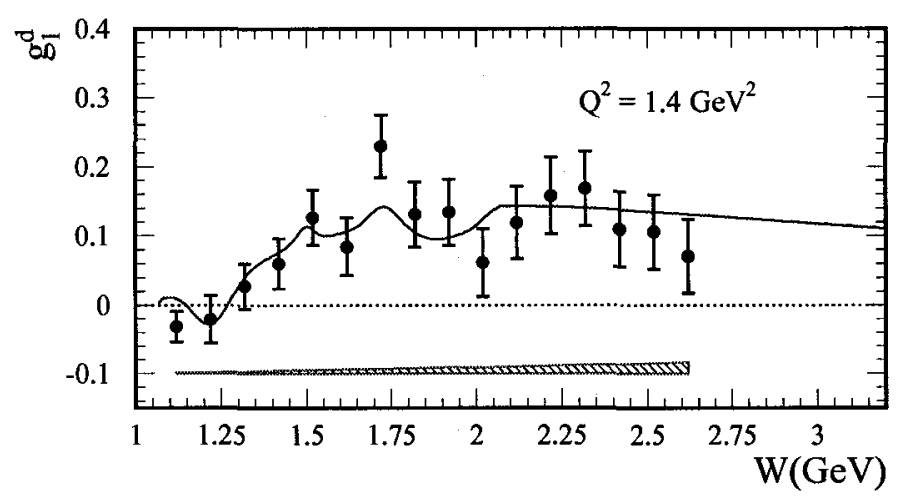

FIG. 101. The total systematic uncertainty due to models used to extract asymmetries compared to the statistical uncertainty for one $Q^{2}$ bin. The systematic error is given as a shaded band at the bottom of the plot.

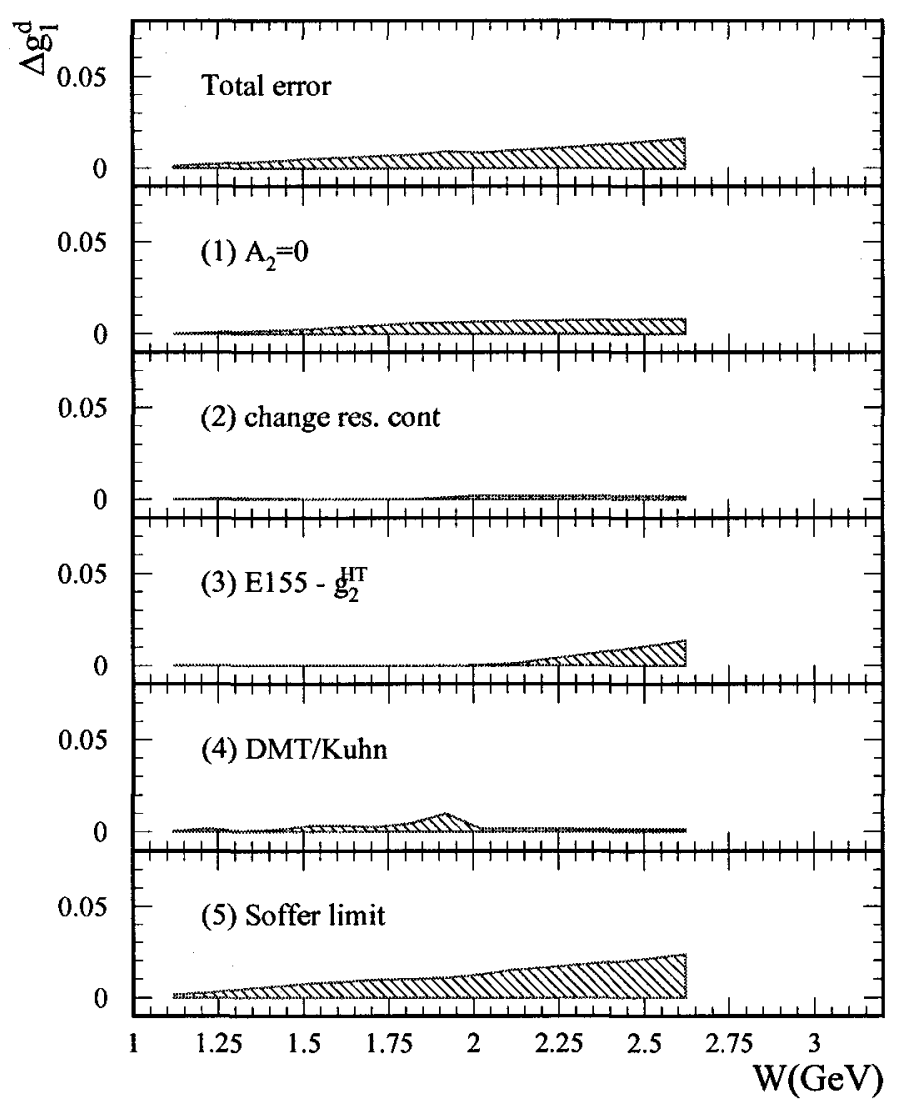

FIG. 102. The total systematic error given in Fig. 101, decomposed into different models used to determine the total error. More details are in the text. 


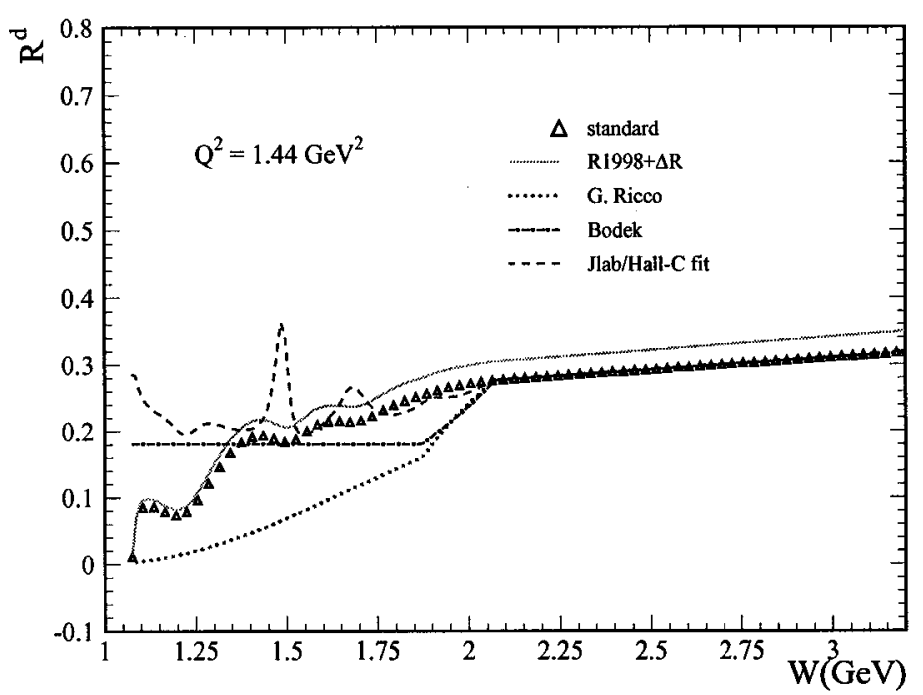

FIG. 103. Different parameterizations of $R$ used to calculate systematic errors. More details are in the text.

- The uncertainty in the standard parameterization of $R, \mathrm{R} 1998$ [72], given by equation (205).

- The model dependence, taken as the variance between the three parameterizations of $R$ (see section 5.8).

- A possible systematic uncertainty in the extraction of $R$ from data, which takes into account the recent revelation from Jefferson lab Hall A that there may be an $\epsilon$-dependent radiative effect (two photon emission).

These uncertainties were added in quadrature and added to the standard value of $R$ to form one of our alternatives $(\mathrm{R} 1998+\Delta R)$.

2. An old parameterization of $F_{1}$ and $F_{2}$ performed by SLAC [75] instead of the NMC parameterization in the DIS region. The data used in the fit include all deep inelastic electron-hydrogen and electron-deuterium measurements at SLAC taken between 1970 and 1983 and cover the kinematic range $0.06 \leq x \leq$ 0.9 and $0.6 \leq Q^{2} \leq 30 \mathrm{GeV}^{2}$ (Fig. 104).

3. G. Ricco's parameterization of $R$ in the resonance region [76].

4. Bodek's parameterization [77] for both $F_{1}$ and $R$ in the resonance region. The parameterization contains a modulating function, which contains twenty four 
parameters, and another twelve parameter function, which describes the deep inelastic region. The modulating function takes into account the masses, widths and amplitudes of the four most prominent nucleon resonances and the $W$ dependence of the nonresonant background under these resonances. The parameters were determined by doing a least square fit to the data given in [78].

5. New parameterization of $F_{1}, F_{2}$ and $R$ by Jefferson lab, Hall-C [79]. The data used for the fit cover a kinematic range of $1<W<2 \mathrm{GeV}$ and $0.2<Q^{2}<4$ $\mathrm{GeV}^{2}$. This parameterization is only valid for protons and for $Q^{2}$ above 0.5 $\mathrm{GeV}^{2}$ region. The neutron value was obtained by scaling the standard values [62].

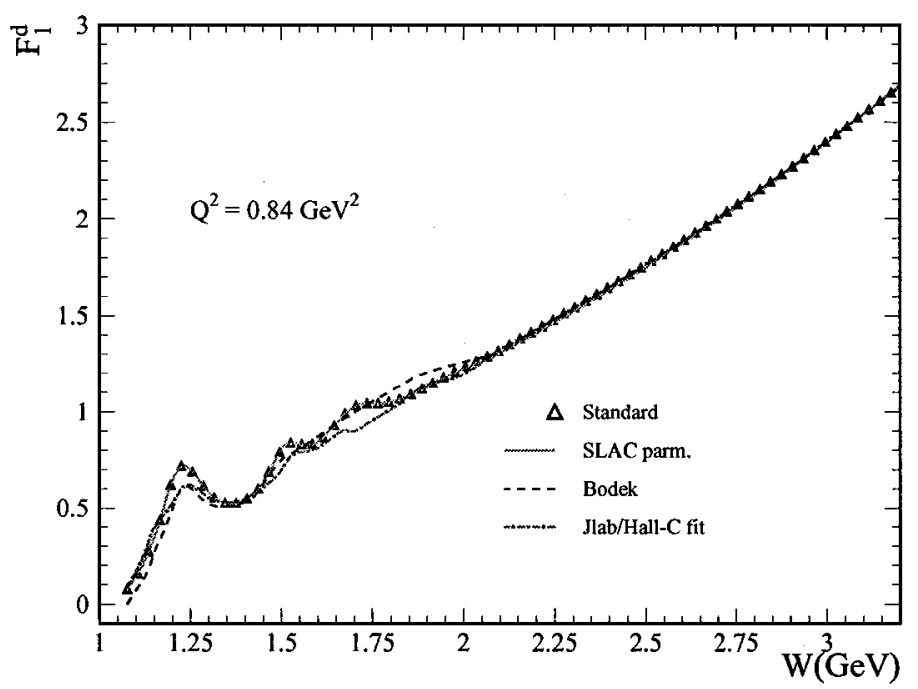

FIG. 104. Different parameterizations of $F_{1}$ used to calculate systematic errors. More details are in the text.

The overall systematic uncertainty was again calculated using equation 212 . The total was divided by three since all the quantities, $F_{1}, F_{2}$ and $R$ were varied.

Fig. 105 shows the total systematic error due to the modeling of unpolarized structure functions to extract our final results. Fig. 106 shows the contribution from each of the different models that was used in determining the total error. The systematic error in the resonance region is approximately $5 \%$ and it is less than $1 \%$ in the DIS region. The large error in the resonance region is mainly due to the lack of world data on the unpolarized structure functions in that region.

The final step in the analysis is the extraction of final results. The final results are then compared to available theoretical predictions. A description of the method by 


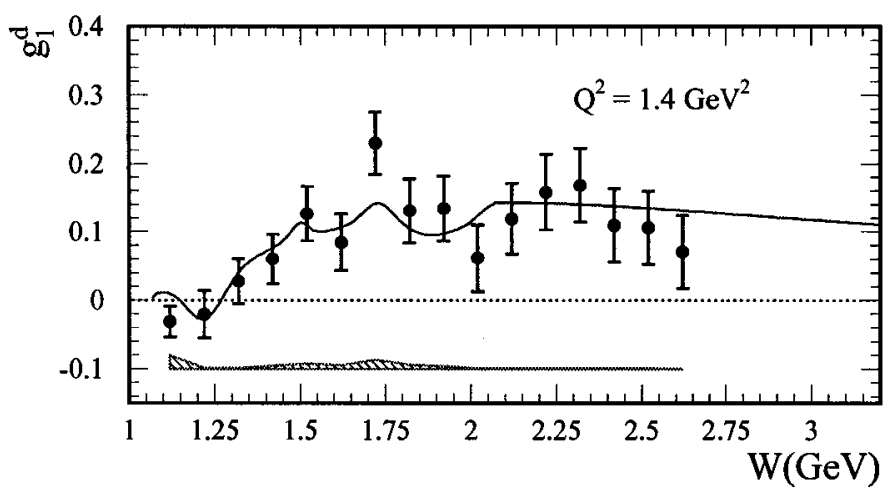

FIG. 105. The total systematic uncertainty due to modeling of structure functions compared to the statistical uncertainty for one $Q^{2}$ bin. The systematic error is given as a shaded band at the bottom of the plot.

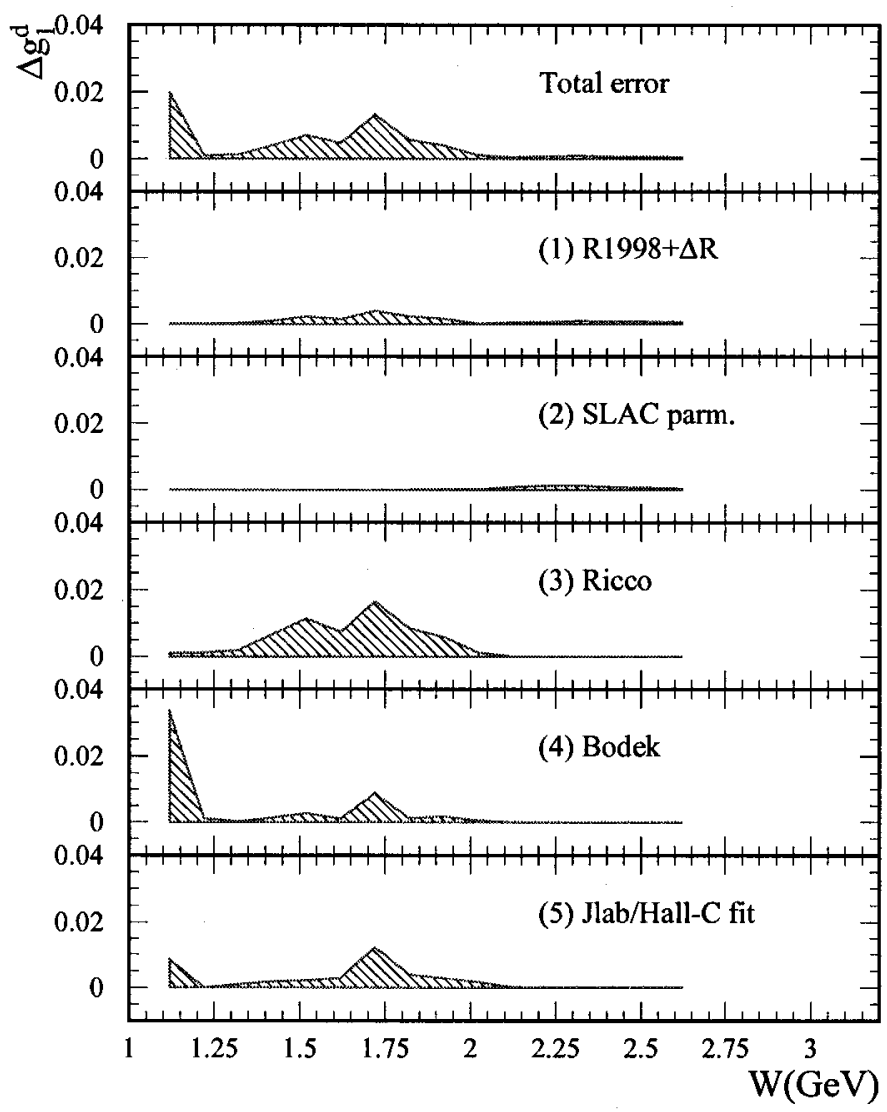

FIG. 106. The total systematic error given in Fig. 105, decomposed into different models used to determine the total error. More details are in the text. 
which the final results were extracted and a discussion on the comparison to theory will be given in the next section. 


\section{CHAPTER 6}

\section{RESULTS}

The main goal of the experiment is to extract the physics quantities $A_{1}, g_{1}$ and $\Gamma_{1}$. Once the final measured asymmetry, $A_{\|}$, is calculated using the methods described in the previous chapter $A_{1}$ and $g_{1}$ can be extracted. In this chapter details about how these physics quantities were extracted and what we can learn from them will be discussed.

\subsection{ASYMMETRY $A_{1}+\eta A_{2}$}

The asymmetry $A_{1}+\eta A_{2}$ can be extracted by dividing the asymmetry $A_{\|}$by the depolarization factor $D$,

$$
D=\frac{1-\epsilon \frac{E^{\prime}}{E}}{1+\epsilon R}
$$

The parameter $R$ was calculated using the model described in the previous section (hereafter referenced to as the $A_{1}-A_{2}$ parameterization). Fig. 107 shows the

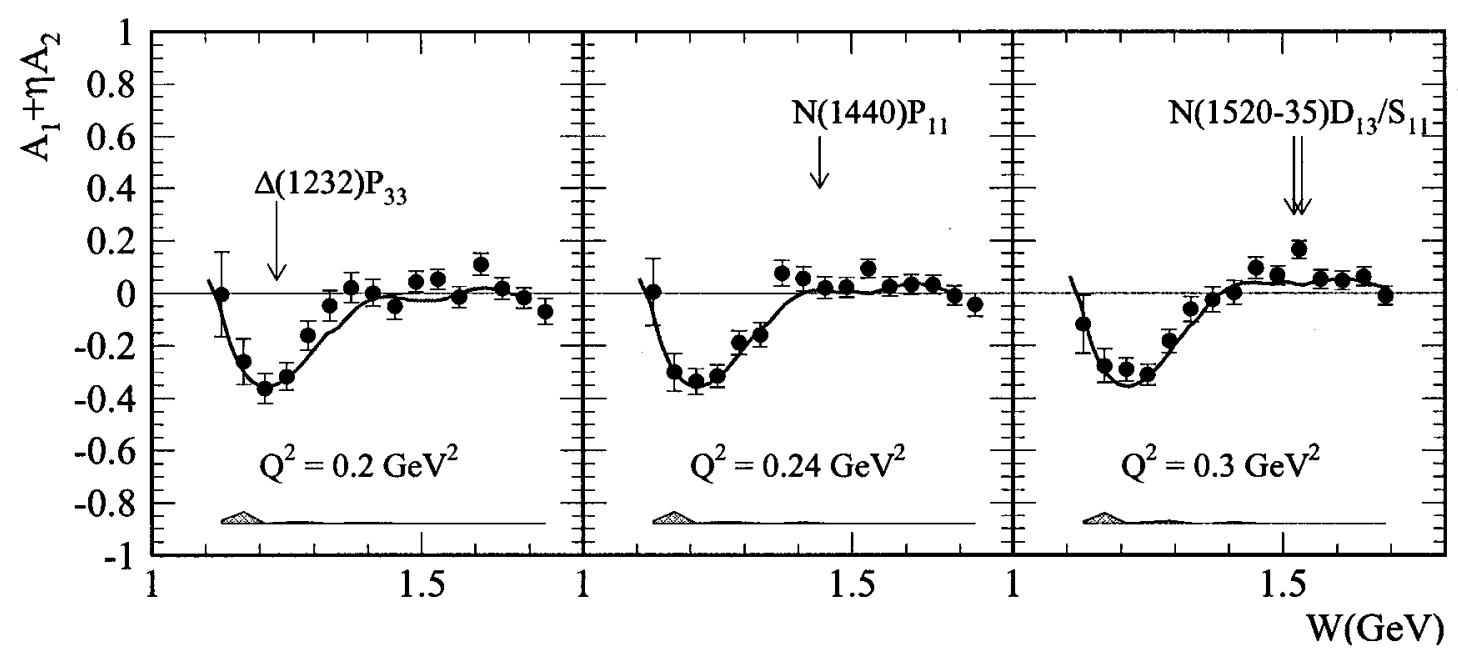

FIG. 107. The asymmetry $A_{1}+\eta A_{2}$ for the $1.6 \mathrm{GeV}$ data. The positions of some of the main resonances are indicated by arrows. The line is the parameterization of $A_{1}$ and $A_{2}$ discussed in the previous section. The error bars show statistical uncertainties. The shaded region at the bottom of the plot indicates the systematic uncertainties.

asymmetry obtained for a few $Q^{2}$ bins for the $1.606 \mathrm{GeV}$ data. These are the first deuteron measurements in that kinematic region. The asymmetry is negative for the 
$\Delta$ resonance and the value is close to -0.5 , which is the value expected for $A_{1}$ for a pure magnetic dipole transition to the $\Delta$ resonance. The asymmetry is close to zero in the high $W$ region. However the asymmetry is becoming positive in the $D_{13}$ and $S_{11}$ region in the $Q^{2}=0.3 \mathrm{GeV}^{2}$ bin. Fig. 108 shows $A_{1}+\eta A_{2}$ for a few $Q^{2}$ bins for the $5 . \mathrm{x} \mathrm{GeV}$ data. At these large $Q^{2}$ the asymmetry for the $\Delta$ resonance is consistent with zero and at high $W$ the asymmetries are positive and large, indicating that the amplitude corresponding to the absorption cross section $\sigma_{1 / 2}^{T}$ is the dominant one. It is also evident that the asymmetries in the $D_{13}$ and $S_{11}$ region shows only a little change with increasing $Q^{2}$. Also shown in the figures is the asymmetry, $A_{1}+\eta A_{2}$, calculated using the model. The data agree with those predictions quite well.

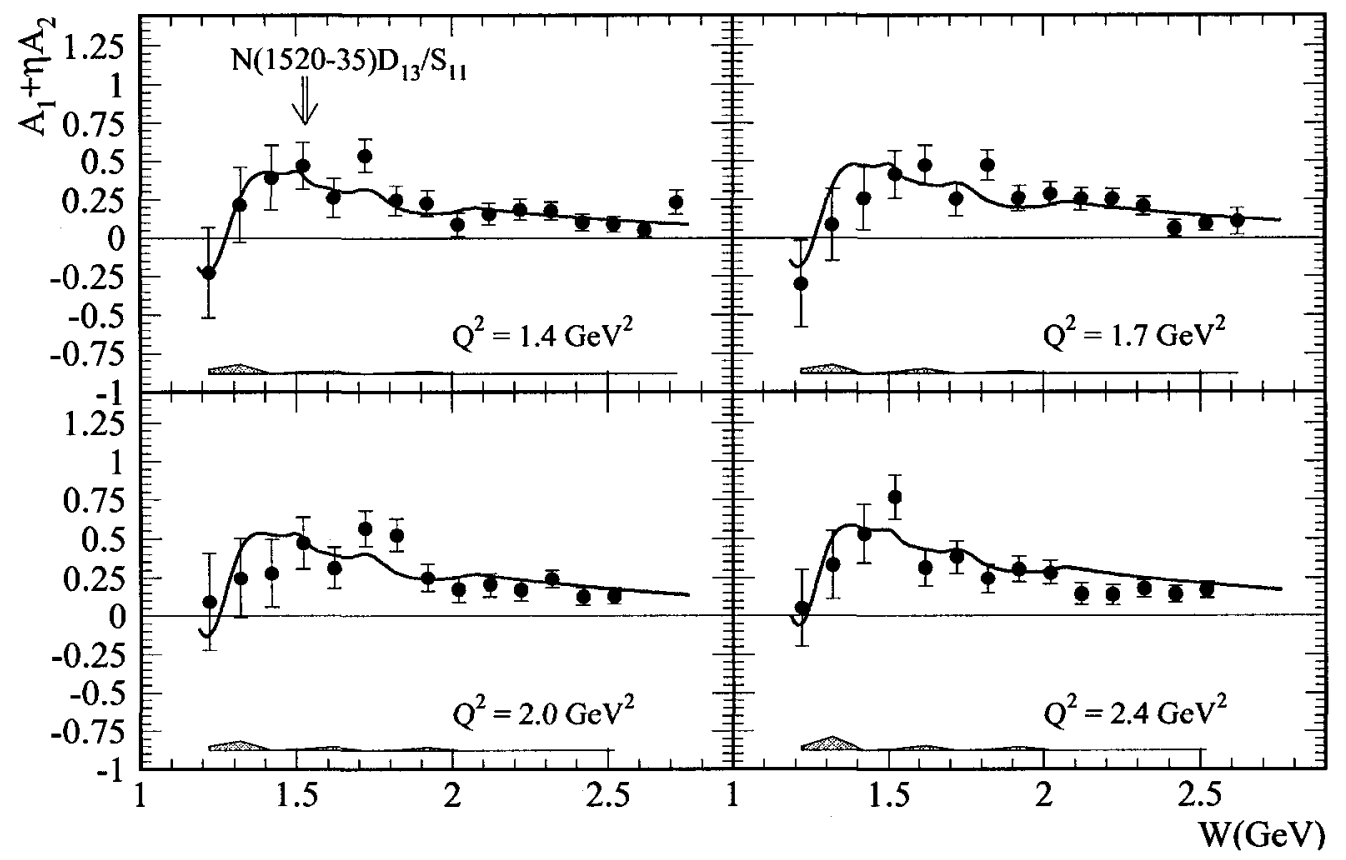

FIG. 108. The asymmetry $A_{1}+\eta A_{2}$ for $5 . \mathrm{x} \mathrm{GeV}$ data. The data were combined assuming the factor $\eta A_{2}$ is small and is the same for all different $5 . \mathrm{x} \mathrm{GeV}$ beam settings. The line is the $A_{1}-A_{2}$ parameterization. The error bars show statistical uncertainties. The shaded region at the bottom of the plot indicates the systematic uncertainties.

As discussed before $A_{1}$ or $A_{2}$ cannot be extracted directly from the measured asymmetries. Fig. 109(a) compares $\eta A_{2}$ calculated using the model with the full measured asymmetry $A_{1}+\eta A_{2}$ for one $Q^{2}$ bin. Since the asymmetry $A_{2}$ is constrained by $\left|A_{2}\right| \leq \sqrt{\frac{1}{2}\left(A_{1}+1\right) R}$, the contribution of the $\eta A_{2}$ part to the full asymmetry is small as expected implying that $A_{1}+\eta A_{2} \approx A_{1}$. 

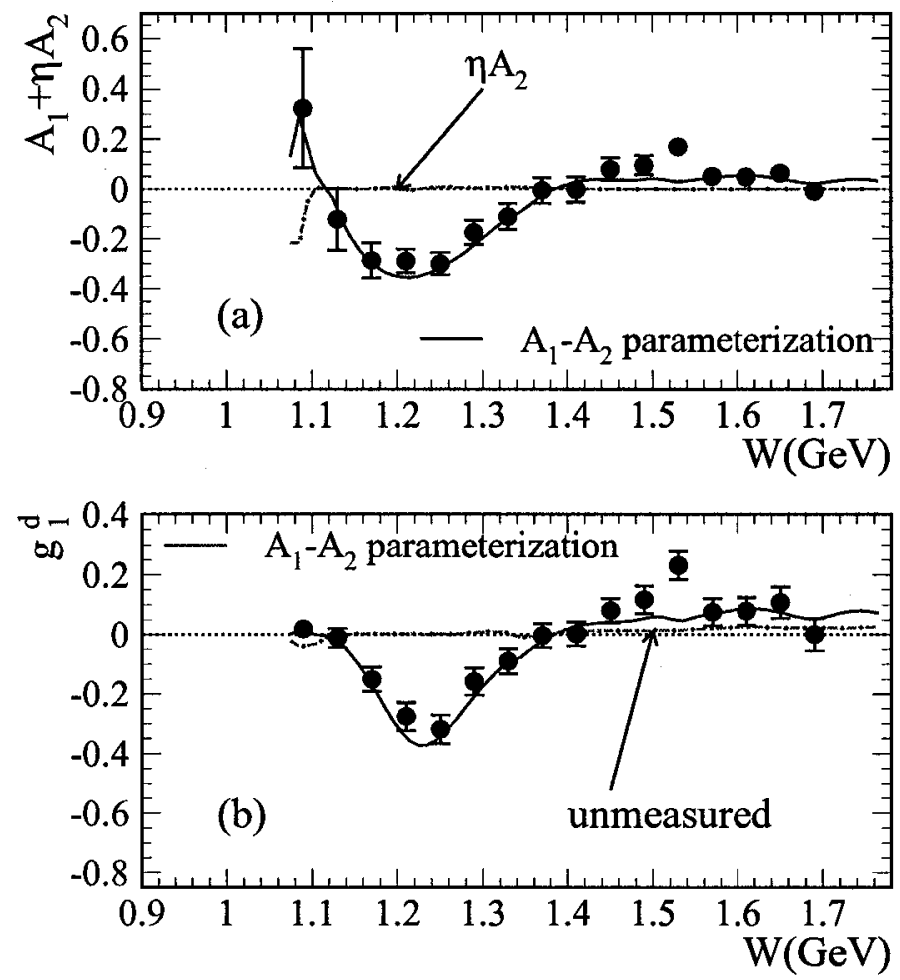

FIG. 109. The figure compares $g_{1}$ and $A_{1}$ with the contribution from the unmeasured part. (a) The contribution of $\eta A_{2}$ to the asymmetry $A_{1}+\eta A_{2}$ for $Q^{2}=0.3 \mathrm{GeV}^{2}$ is plotted with the measured asymmetry. (b) The unmeasured contribution to $g_{1}$ along with the finally extracted results for $g_{1}$ as a function of $W$ for the same $Q^{2}$ bin is shown. Data are taken with a beam energy of $1.6 \mathrm{GeV}$.

\subsection{SPIN STRUCTURE FUNCTION $g_{1}$}

Since $A_{1}$ cannot be separated from $A_{2}, g_{1}$ was extracted by adding the unmeasured part to the measured asymmetry $A_{\|} / D$,

$$
g_{1}=\frac{\tau}{1+\tau}\left[\frac{A_{\|}}{D}+\left(\frac{1}{\sqrt{\tau}}-\eta\right) A_{2}\right] F_{1} .
$$

A knowledge of $F_{1}$ is also required to extract $g_{1}$, and was parameterized using the methods described in section 5.8. Fig. 109(b) compares $g_{1}$ with the contribution from the unmeasured part.

The value of $g_{1}$ was first measured for each of the data sets separately and then was combined after doing a z-score test. The z-score values of $g_{1}$ for different data 

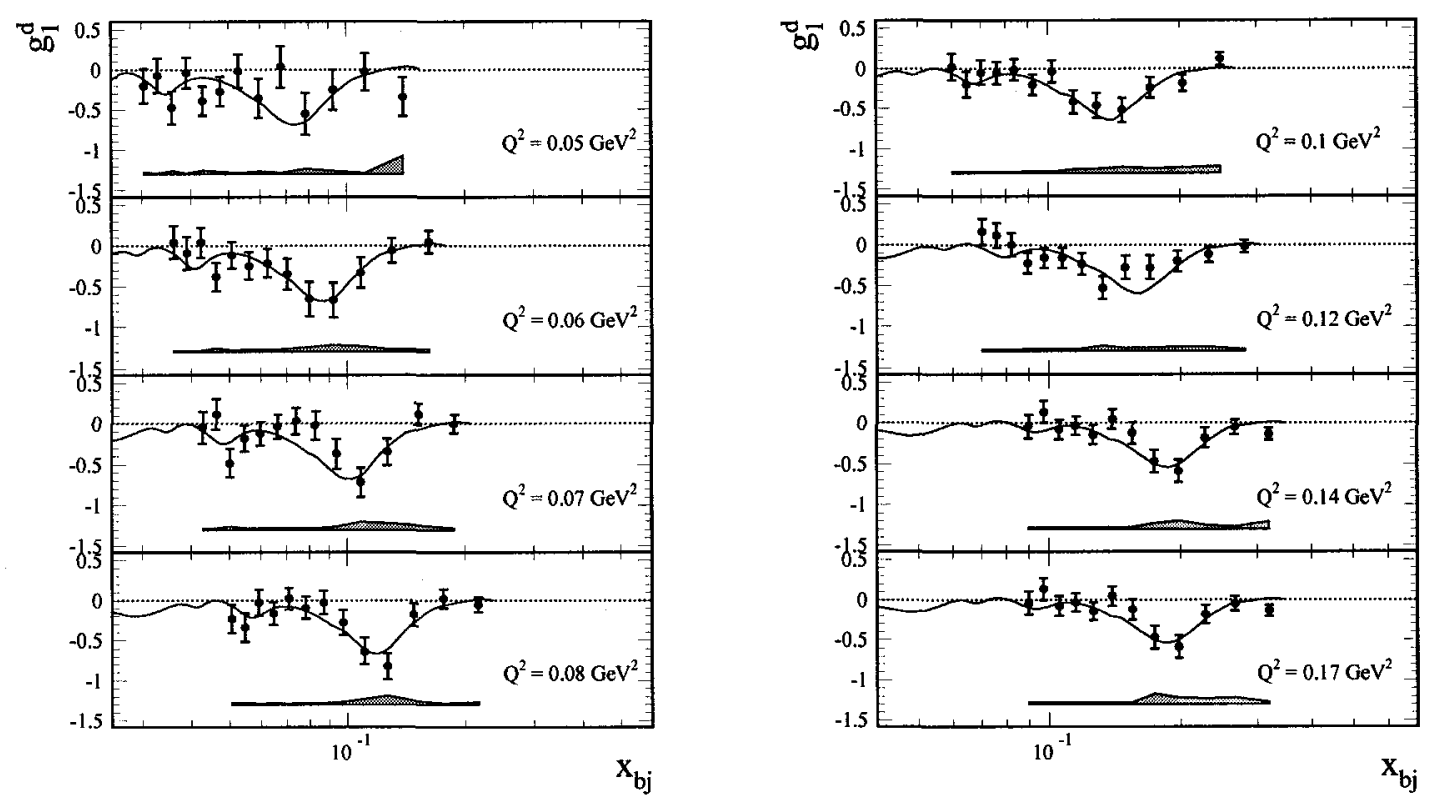

FIG. 110. $g_{1}$ as a function of $x$ for $Q^{2}=0.05-0.17 \mathrm{GeV}^{2}$. The line is the $A_{1}-A_{2}$ parameterization. The error bars show statistical uncertainties and the shaded band at the bottom of each plot indicates the systematic uncertainties.

sets are listed in section 5.9. The value of $g_{1}$ for different data sets agree very well. The combined results for $g_{1}^{d}$ for all the $Q^{2}$ bins are listed with the corresponding statistical and systematic errors in Tables from XIII to XXXVI. Fig. 110, 111 and 112 show $g_{1}$ as a function of $x$ for different $Q^{2}$ bins. The negative contribution of the $\Delta$ resonance is evident at low $Q^{2}$ values. At large $Q^{2} g_{1}$ is large and less $Q^{2}$ dependent.

\subsection{DUALITY}

As mentioned in chapter two, one important thing that can be studied with the resonance data is duality. Fig. 113 compares $g_{1}$ versus $\xi$ with the scaling structure function at $Q^{2}=10 \mathrm{GeV}^{2}$, which was obtained using the $A_{1}-A_{2}$ parameterization and turning off the resonance contribution. At large $Q^{2}$ values the data resemble the scaling structure function very well. However at lower $Q^{2}$ values there is a noticeable difference between the data and the scaling structure function, particularly in the region of the $\Delta$ excitation. To quantify these observations the data has to be integrated over the resonance region and compared to the scaling structure function 

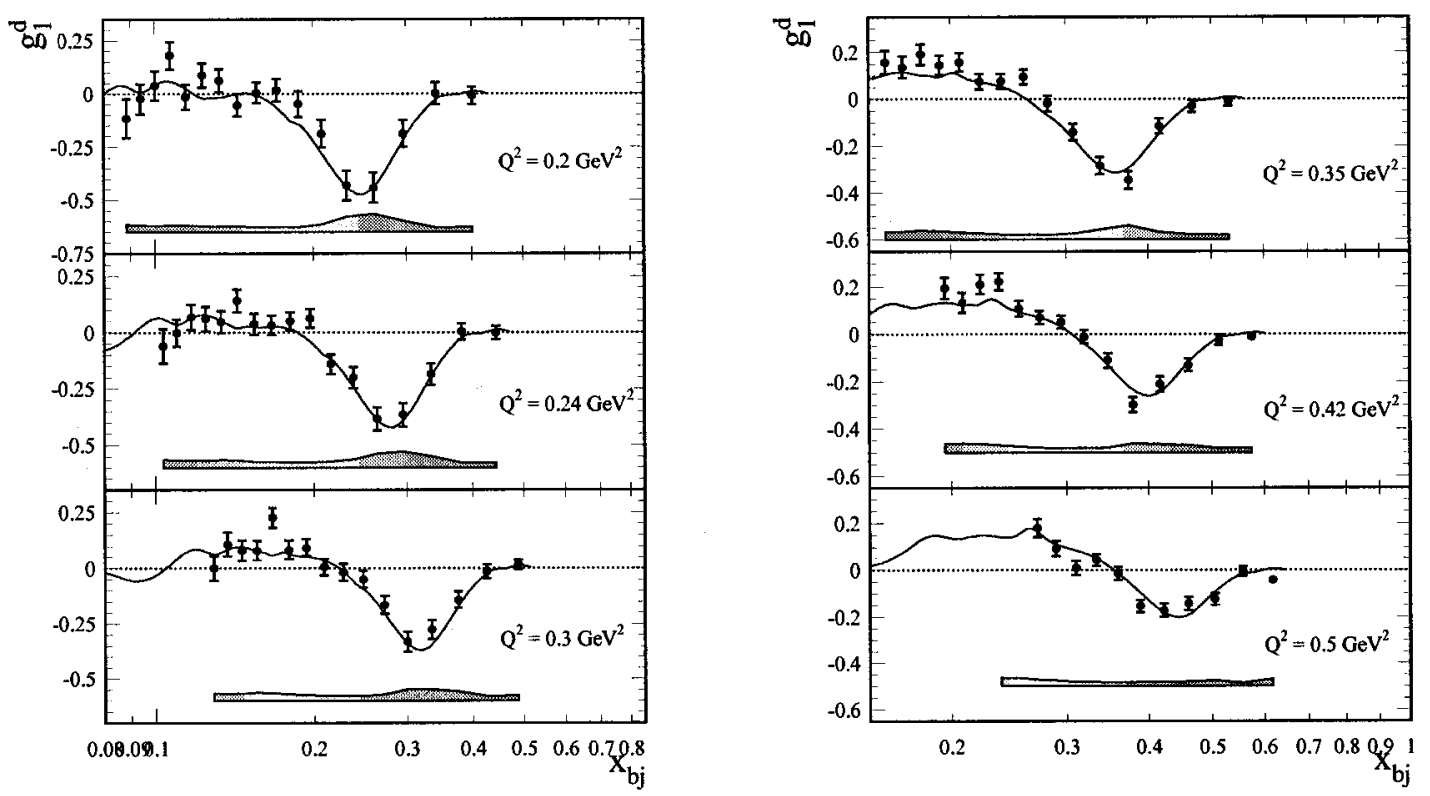

FIG. 111. Same as Fig. 110 but for $Q^{2}=0.2-0.5 \mathrm{GeV}^{2}$.
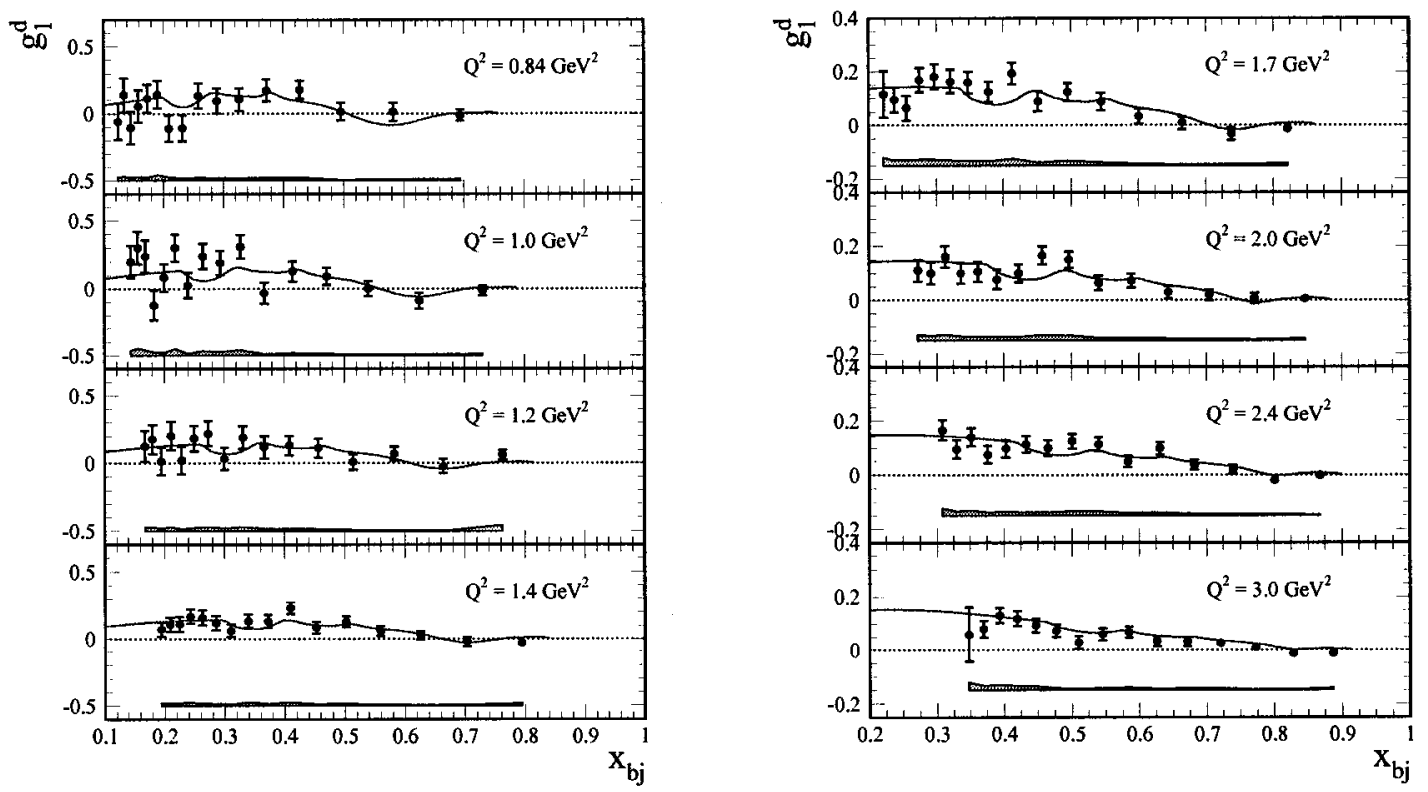

FIG. 112. Same as Fig. 110 but for $Q^{2}=0.84-3.0 \mathrm{GeV}^{2}$. 

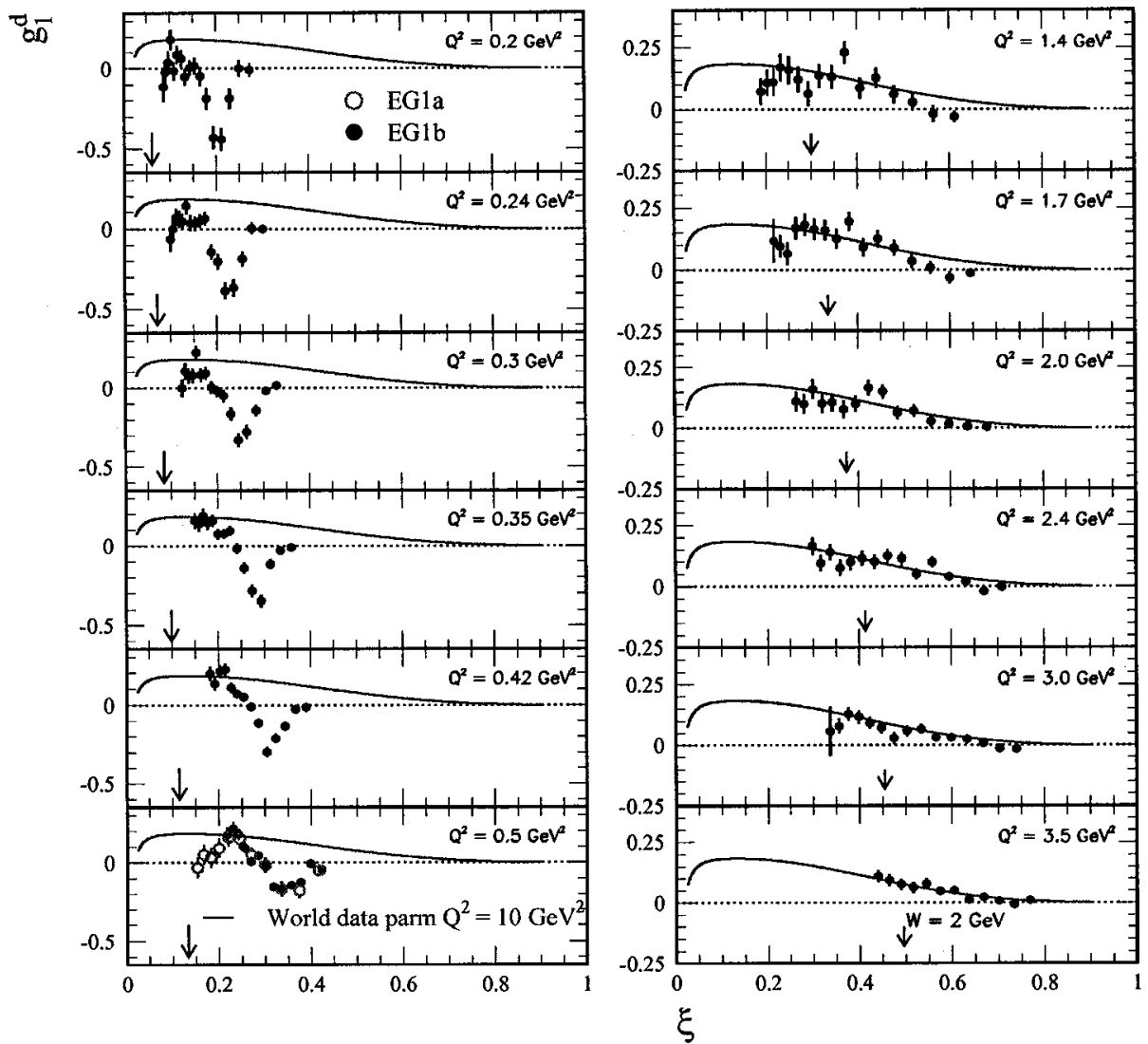

FIG. 113. Spin structure function $g_{1}$ as a function of the Natchmann variable $\xi$. Data are compared with the scaling structure function at $Q^{2}=10 \mathrm{GeV}^{2}$. The arrow indicates the $W=2 \mathrm{GeV}$ point. The open circles are data from the first part of this same experiment [40]. Errors include only the statistical error.

in the same $\xi$ region,

$$
I_{\text {res } / D I S}\left(Q^{2}\right)=\frac{\int_{\xi \min }^{\xi \max } g_{1}^{\text {data }}\left(\xi, Q^{2}\right) d \xi}{\int_{\xi \min }^{\xi \max } g_{1}^{s c a l i n g}\left(\xi, Q^{2}\right) d \xi} .
$$

The following four $W$ regions were selected as $\xi \min$ and $\xi \max$ :

1. $1.07 \mathrm{GeV}<W<2 \mathrm{GeV}$ which includes the entire resonance region.

2. $1.12 \mathrm{GeV}<W<1.38 \mathrm{GeV}$ which includes the $\Delta(1232)$ region.

3. $1.38 \mathrm{GeV}<W<1.58 \mathrm{GeV}$ which includes the $\mathrm{S}_{11}$ (1535) region.

4. $1.58 \mathrm{GeV}<W<1.8 \mathrm{GeV}$ which includes the $\mathrm{F}_{15}(1680)$ region. 
Since the $Q^{2}=10 \mathrm{GeV}^{2}$ curve is mostly dominated by the leading order (LO) term, to understand duality at lower $Q^{2}$ the scaling curve has to be properly evolved down to the $Q^{2}$ value of the particular bin of interest. The evolution of the scaling

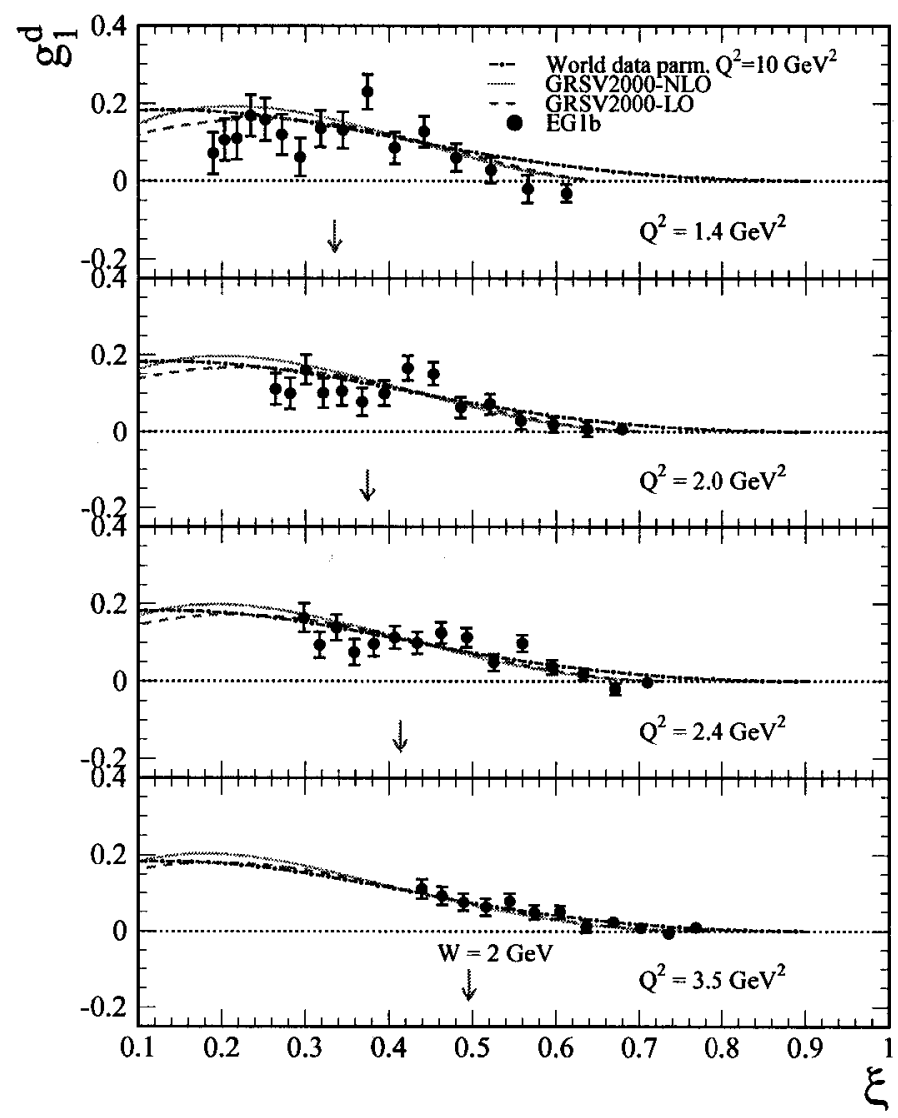

FIG. 114. A comparison between the scaling structure function calculated using the GRSV2000 LO and NLO calculations [80] and a parameterization of world data at $Q^{2}=10 \mathrm{GeV}^{2}$. More details are in the text. Errors include only the statistical error.

structure function calculated in Next to Leading Order (NLO) has been studied and formulated by M. Glueck, E. Reya, M. Stratmann and W. Vogelsang (GRSV2000) [80]. As a systematic check the scaling structure function was integrated using the $Q^{2}=10 \mathrm{GeV}^{2}$ curve explained above and the LO and NLO GRSV2000 scaling curves properly evolved down to the $Q^{2}$ bin of interest. Fig. 115 shows the difference between these three curves for a few $Q^{2}$ bins. A comparison between the data integrated in the entire resonance region divided by the scaling structure function calculated using the above mentioned three cases is given in Fig. 115. The use of the LO or the NLO calculation for the scaling structure function did not make a significant difference in the ratio $I_{R E S / D I S}$. At large $Q^{2}$ values the ratio is approximately equal 
to one confirming the presence of duality in the resonance region. However when we go to lower $Q^{2}$ values the ratio becomes less than one since the negative contribution due to the Delta resonance becomes important.

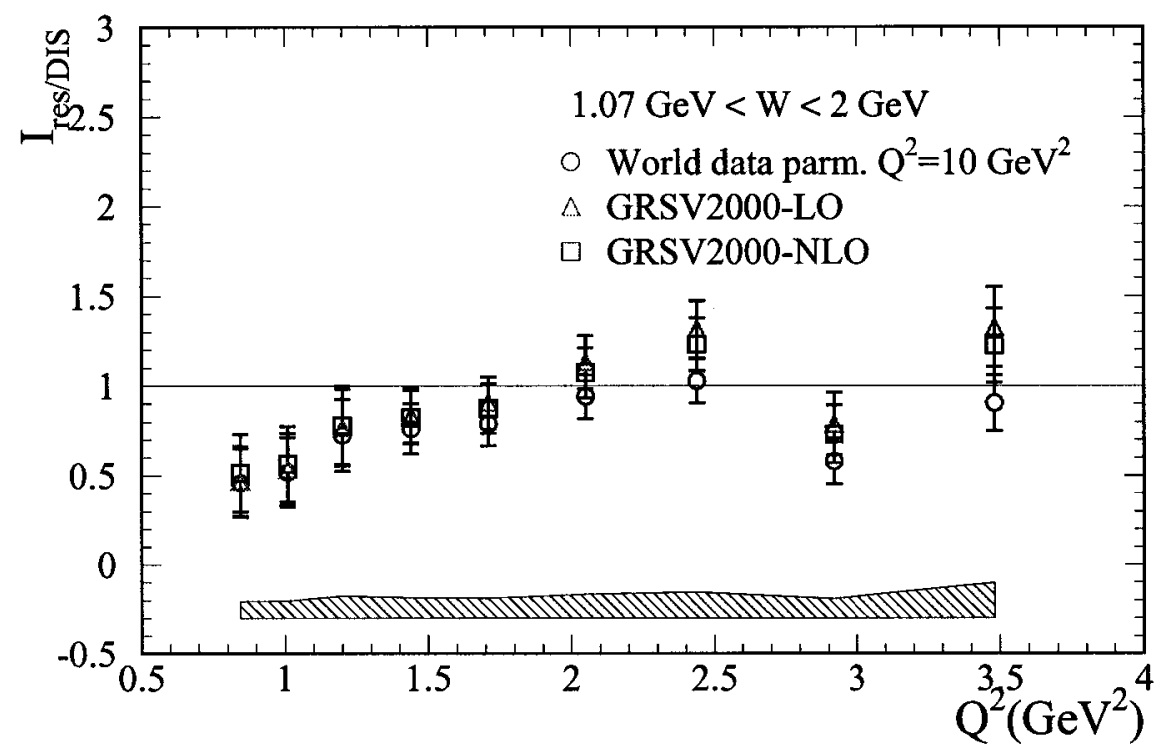

FIG. 115. The ratio $I_{R E S / D I S}$ in the entire resonance region. The different symbols correspond to different methods used in the calculation of the denominator. The error bars show statistical uncertainties. The shaded region at the bottom of the plot indicates the systematic uncertainties.

To further study this local form of duality the data in the three prominent resonance regions given above were compared to the corresponding scaling value. Data were only compared to the scaling structure function calculated in NLO evolved to the same $Q^{2}$ for each bin. Fig. 116 shows the observed results. In the $\Delta$ region the ratio is below unity as expected. For higher resonances the ratio is unity even at $Q^{2}$ values as low as $\approx 0.8 \mathrm{GeV}^{2}$ (the NLO calculations only go down to $0.8 \mathrm{GeV}^{2}$ ). In conclusion the results indicate that duality holds in the resonance region even at somewhat low $Q^{2}$ with the notable exception of the $\Delta$ region. However, to verify higher twist effects, all final states including the elastic region have to be included. Since we are only comparing the data in the resonance region to the corresponding scaling value, if the elastic region is added to the resonance data, the elastic scattering could show an unrealistically large higher twist contribution. Therefore, to evaluate higher twist contributions, the data must be integrated over the entire $\xi$ region as required by QCD. More details about those effects will be discussed in section 6.6.2. 


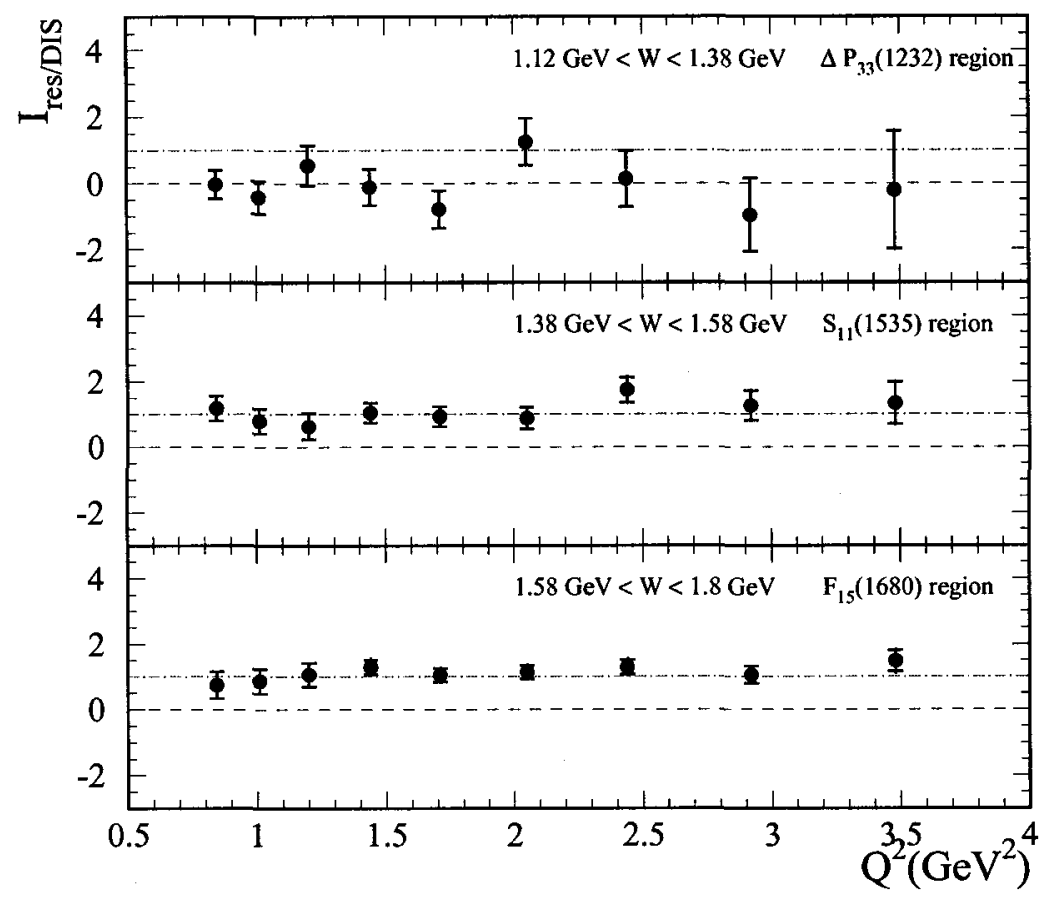

FIG. 116. The ratio $I_{R E S / D I S}$ calculated in the three prominent resonance enhancement regions given in the text. The denominator has been calculated using the GRSV2000 parameterization in NLO.

\subsection{RATIO $g_{1} / F_{1}$}

Fig. 117 shows the ratio $g_{1} / F_{1}$ as a function of $Q^{2}$ averaged into the $5 x$ bins listed in Table XII. Data from the SLAC E154 and E155 experiments are also included in the plot. The dashed line is a fit to SLAC data [68],

$$
\begin{aligned}
& \frac{g_{1}^{p}}{F_{1}^{p}}=x^{0.700}\left(0.817+1.014 x-1.489 x^{2}\right)\left(1-\frac{0.04}{Q^{2}}\right) \\
& \frac{g_{1}^{n}}{F_{1}^{n}}=x^{-0.335}\left(-0.013-0.330 x+0.761 x^{2}\right)\left(1+\frac{0.13}{Q^{2}}\right)
\end{aligned}
$$

combined using,

$$
\frac{g_{1}^{d}}{F_{1}^{d}}=\frac{1-1.5 \omega_{D}}{F_{1}^{n}+F_{1}^{p}}\left(g_{1}^{p}+g_{1}^{n}\right) .
$$

The ratio was calculated using,

$$
\frac{g_{1}}{F_{1}}=\frac{\tau}{1+\tau}\left[\frac{A_{\|}}{D}+\left(\frac{1}{\sqrt{\tau}}-\eta\right) A_{2}\right],
$$




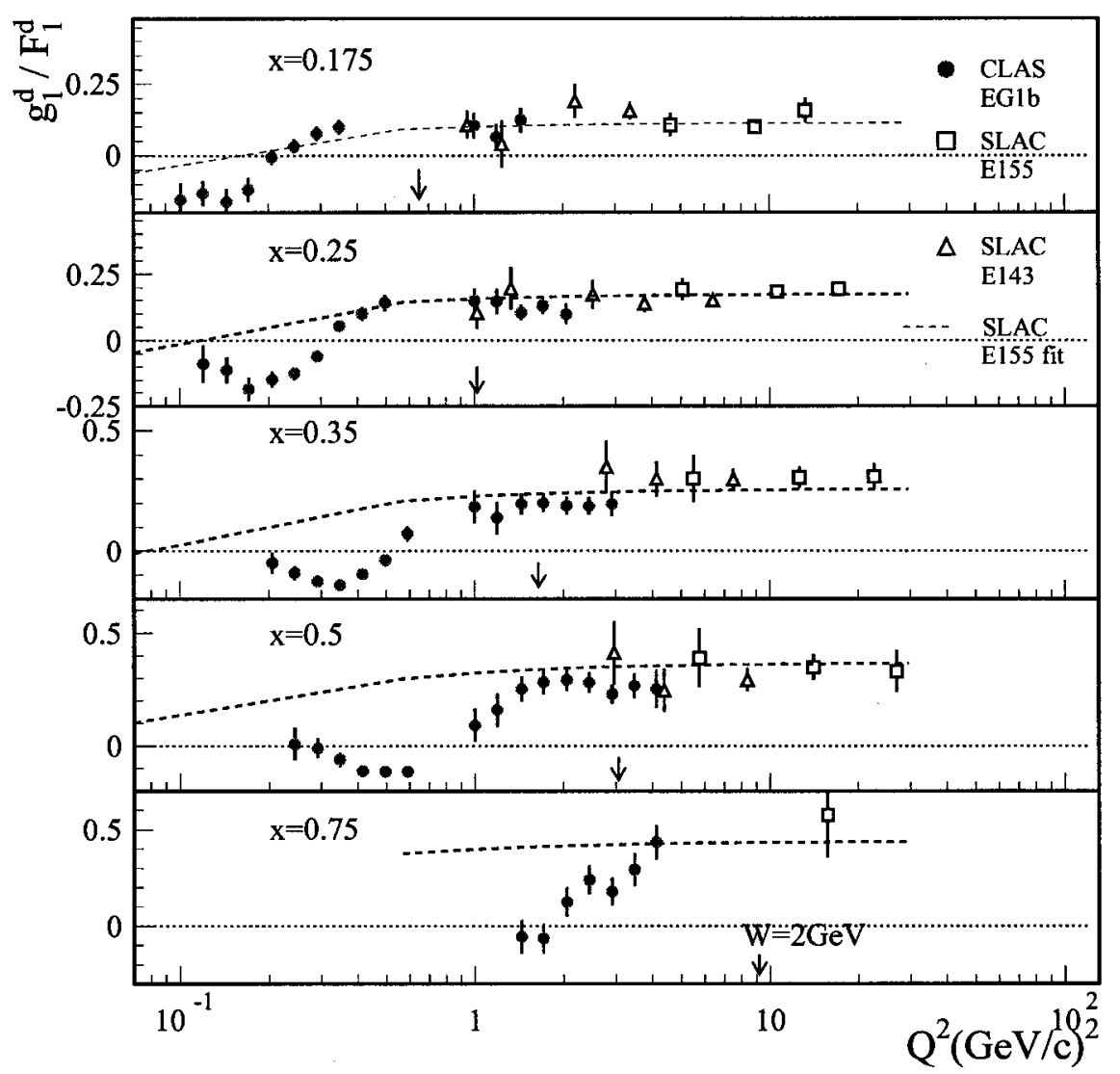

FIG. 117. The figure shows the $Q^{2}$ evolution of the ratio $g_{1} / F_{1}$ for the deuteron. Details about the plot are in the text.

TABLE XII. The upper and the lower limits of the $x$ bins used in the calculation of $g_{1} / F_{1}$.

\begin{tabular}{|c|c|c|}
\hline label & lower limit & upper limit \\
\hline 0.175 & 0.15 & 0.2 \\
0.250 & 0.20 & 0.3 \\
0.350 & 0.30 & 0.4 \\
0.500 & 0.40 & 0.6 \\
0.750 & 0.60 & 0.9 \\
\hline
\end{tabular}


and requires less model input to extract from the measured asymmetry. The results are listed in Tables XXXVII and XXXVIII. At large $Q^{2}$ values the ratio shows very little $Q^{2}$ dependence and agrees well with the SLAC fit mentioned above. The $Q^{2}$ dependent coefficients in the fit for the proton and the neutron are small confirming the relative $Q^{2}$ independence. According to the DGLAP equations $g_{1}$ as well as $F_{1}$ are expected to show a slow logarithmic $Q^{2}$ dependence but the ratio of the two does not show any such dependence, indicating that the $Q^{2}$ evolution of $g_{1}$ is very similar to the $Q^{2}$ evolution of $F_{1}$. However at lower $Q^{2}$ values the resonance structure causes $g_{1}$ to evolve very differently from $F_{1}$ and the ratio goes negative near the $\Delta$ region as expected. In the future our data can be included as part of a new NLO DGLAP fit.

\subsection{VIRTUAL PHOTON ASYMMETRY $A_{1}$}

Fig. 118 shows $A_{1}$ as a function of $x$. All $5 . \mathrm{x} \mathrm{GeV}$ data in the $1.4 \mathrm{GeV}^{2}<Q^{2}<$ $4.52 \mathrm{GeV}^{2}$ region and $W>1.6 \mathrm{GeV}$ region were combined to make the plot. $A_{1}$ was calculated by removing the $\eta A_{2}$ term from the measured asymmetry $A_{\|} / D$. The results are listed in Table XXXIX. The data are compared to the world data parameterized at $Q^{2}=10 \mathrm{GeV}^{2}$. The difference in the small $x$ region may be due to the fact that the data are not entirely in the DIS region. The difference between the data and the $Q^{2}=10 \mathrm{GeV}^{2}$ parameterization in the high $x$ region is mainly due to nuclear binding effects and possibly also due to inclusion of part of the the resonance region ( $W$ between 1.6 and $2 \mathrm{GeV}$ ) in our data. The plot also shows $A_{1}$ calculated by simulating data [62] which includes the deuteron wave function (world data parm $+\mathrm{dWF}$ ). The model that was used in the simulation assumes the proton or neutron inside deuterium is moving with a momentum distributed according to the Paris wave function, and is off-shell in mass-energy so that its energy plus that of the spectator nucleon add up to the deuteron mass. The cross section of the moving nucleon was calculated in a covariant way. The model does not include any quark structure modifications of the moving nucleon. As can be seen at large $x$ there is a difference between $A_{1}$ calculated without including the nuclear binding effects (world data parm $Q^{2}=10 \mathrm{GeV}^{2}$ ) and when it is included. To obtain $A_{1}$ corrected for those nuclear effects the difference has to be added to the data. The data are in good agreement with SLAC E143 data and the statistical precision is much better in the high $x$ region. The data are also compared to the models mentioned in the 
section 2.4. At large $x$ it can clearly be seen that the data are exceeding the $\mathrm{SU}(6)$ quark model predictions. On average $A_{1}$ is small and close to zero at small $x$ and

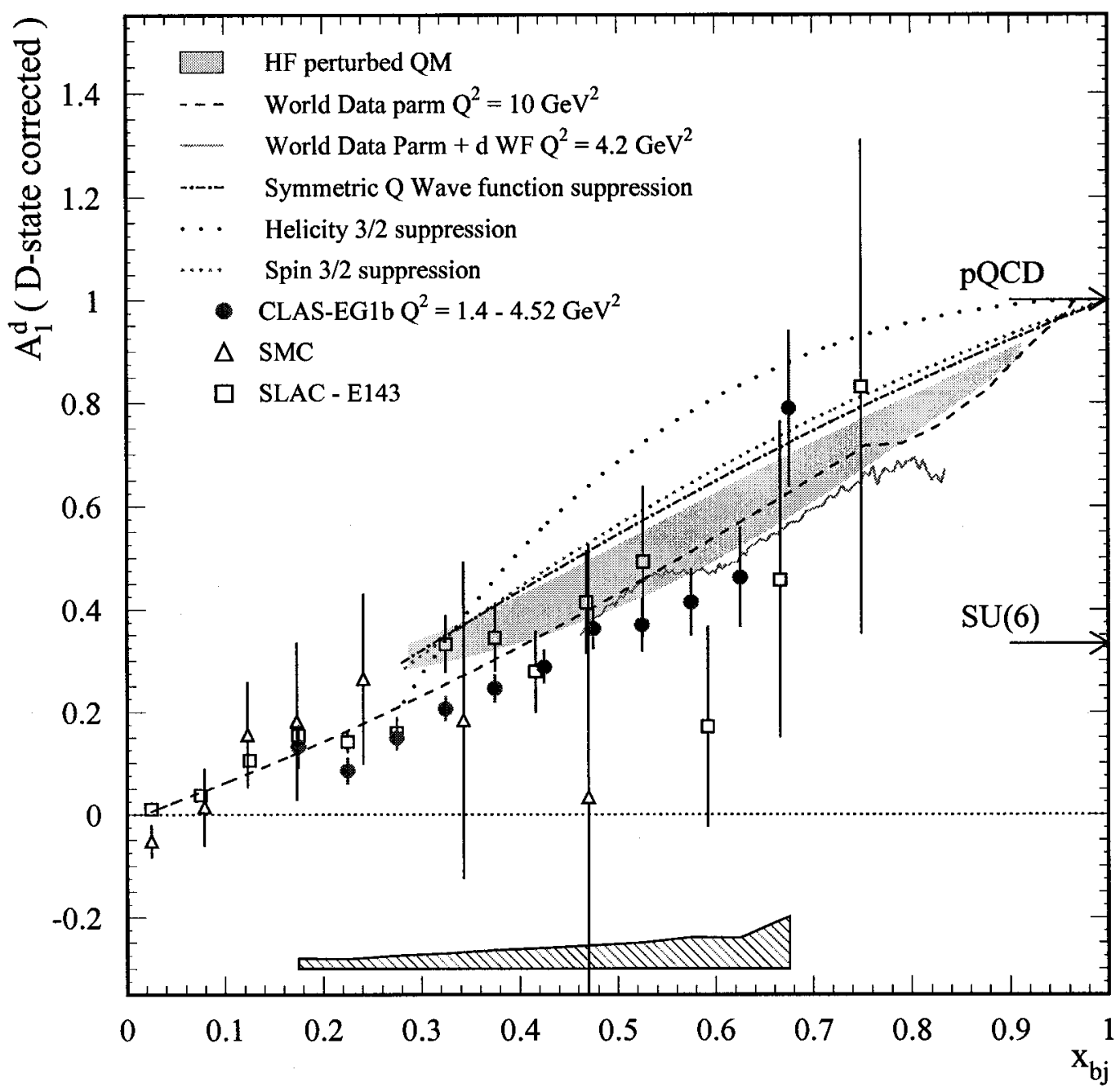

FIG. 118. The virtual photon asymmetry after correcting for the D state as a function of $x$. Details about the plot are in the text. The error bars show statistical uncertainties. The shaded region at the bottom of the plot indicates the systematic uncertainties.

rises towards the $\mathrm{pQCD}$ predictions as $x \rightarrow 1$. The data do not favor the predictions made using duality arguments. However the data are more in agreement with the Hyperfine perturbed quark model (HF perturbed QM) predictions. Our data suggest a negative value for $\frac{\Delta d}{d}$, which is consistent with Jefferson lab Hall-A measurements of $A_{1}$ [81]. To make precise conclusions at large $x$ more data are necessary in that region. 


\subsection{THE FIRST MOMENT OF $g_{1}$}

To calculate the full integral of $g_{1}$ over $x$ from 0 to $x(W=1.07 \mathrm{GeV})$, knowledge of the entire $x$ region for each $Q^{2}$ bin is necessary. However our measurements do

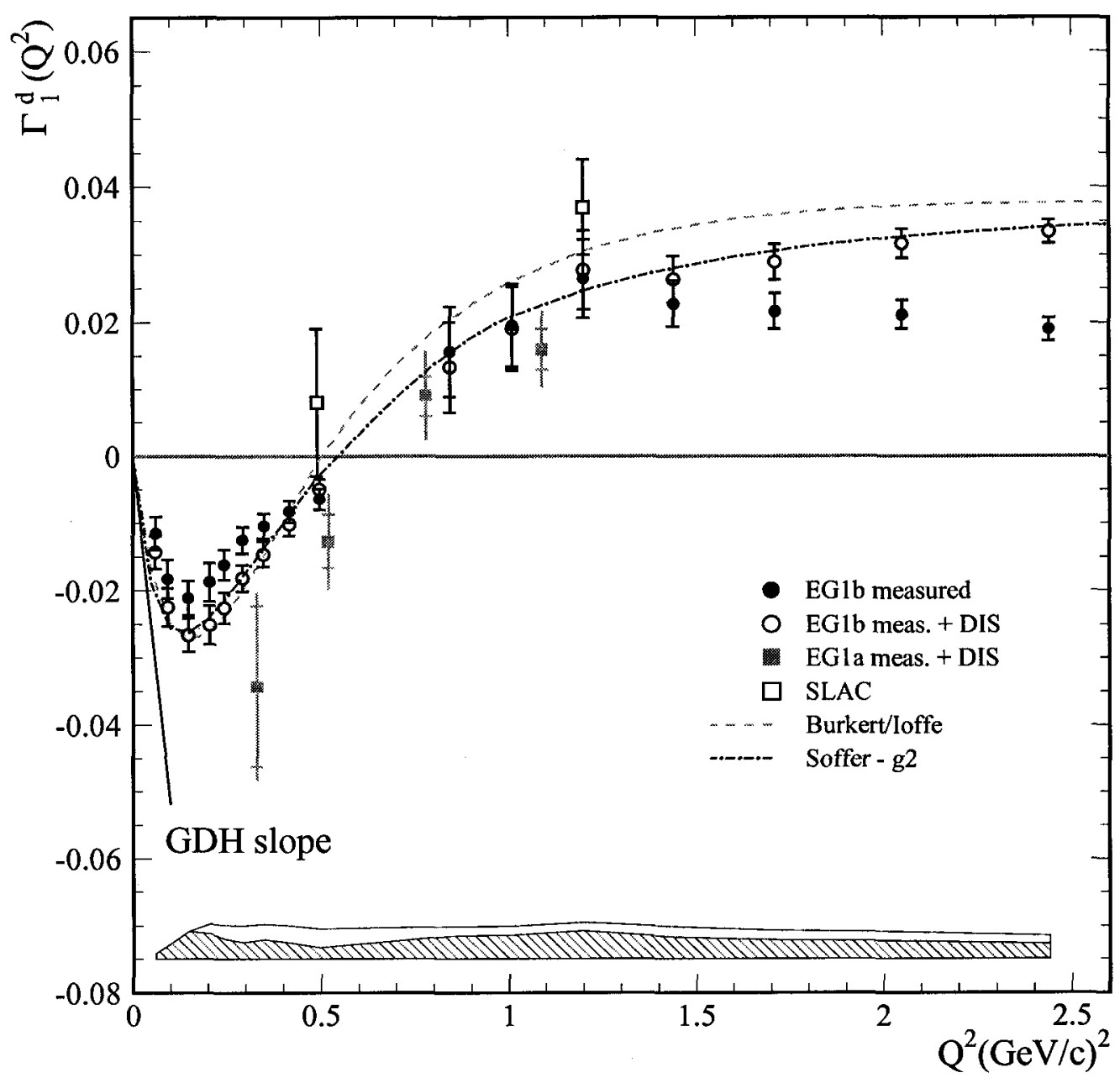

FIG. 119. Comparison of $\Gamma_{1}$ to models described in the second chapter. The error bars show statistical uncertainties. The shaded region and the line above the shaded region at the bottom of the plot indicate the systematic uncertainties for the measured points (EG1b measured) and for the full integral (EG1b meas. + DIS) respectively.

not cover the full $x$ region, so $g_{1}$ in the unmeasured region was calculated using the model described in the previous chapter. Therefore the integral can be divided into two regions,

$$
\Gamma_{1}\left(Q^{2}\right)=\underbrace{\int_{x=0.001}^{x\left(W_{\max }\right)} g_{1}\left(x, Q^{2}\right)}_{\text {unmeasured }}+\underbrace{\int_{x\left(W_{\max }\right)}^{x(W=1.07)} g_{1}\left(x, Q^{2}\right)}_{\text {measured }} .
$$


The $W_{\max }$ value for each $Q^{2}$ bin is listed in the Table VI. Since the extrapolation of the integral below $x=0.001$ is not well known, the contribution below $x=0.001$ was ignored. Only the data for $Q^{2}$ bins in which the contribution from the model is less than $50 \%$ of the full integral are shown in the plots. All $\Gamma_{1}$ values listed and plotted are given per nucleon, i.e, divided by 2. The results are listed in Tables XL and XLI. Fig. 119 compares data to the two models Burkert and Ioffe [6], Soffer and Teryaev [38] explained in the second chapter. The data agree with the predictions made by Soffer and Teryaev in the large $Q^{2}$ region and agree with both models in the low $Q^{2}$ region. The data show a very strong $Q^{2}$ dependence below the $Q^{2} \approx 2$ $\mathrm{GeV}^{2}$ region. The integral is negative below $Q^{2} \approx 0.5 \mathrm{GeV}^{2}$, which is due to the dominance of the $A_{3 / 2}$ amplitude at lower $Q^{2}$ values. At larger $Q^{2} A_{1 / 2}$ becomes the dominant amplitude and the integral becomes positive as the negative contribution due to the $\Delta$ resonance starts to diminish. The data also show a minimum around $Q^{2}=0.15 \mathrm{GeV}^{2}$ and show a trend towards the GDH slope at low $Q^{2}$.

\subsubsection{Low $Q^{2}$ Region}

Fig. 120 shows $\Gamma_{1}$ in the very low $Q^{2}$ region. In addition to the two models, $\Gamma_{1}$ calculated using Chiral perturbation theory [37] at next to leading order in momenta is also shown. The calculation includes the leading order term calculated in the Heavy Baryon chiral perturbation theory, which is vanishing, and the next to leading order term. The effect of the delta resonance at low $Q^{2}$ has not been included in the calculation, although low-energy effective field theories can be used to include other small parameters, such as the mass difference between nucleon and the delta resonance, in the expansion. These effects have been studied and are believed to be small in the low $Q^{2}$ region [82]. The data agree with the chiral perturbation theory calculations for $Q^{2}$ below $0.08 \mathrm{GeV}^{2}$.

\subsubsection{High $Q^{2}$ Region}

In the moderate to high $Q^{2}$ region OPE allows one to identify the size of higher twist terms. To find the size of these higher twist terms, data in the moderate to high $Q^{2}$ region is needed. According to OPE the full integral, including the elastic contribution, can be written as,

$$
\Gamma_{1}\left(Q^{2}\right)+\Gamma(e l)=\mu_{2}+\frac{\mu_{4}}{Q^{2}}+\frac{\mu_{6}}{Q^{4}}+\ldots . .
$$




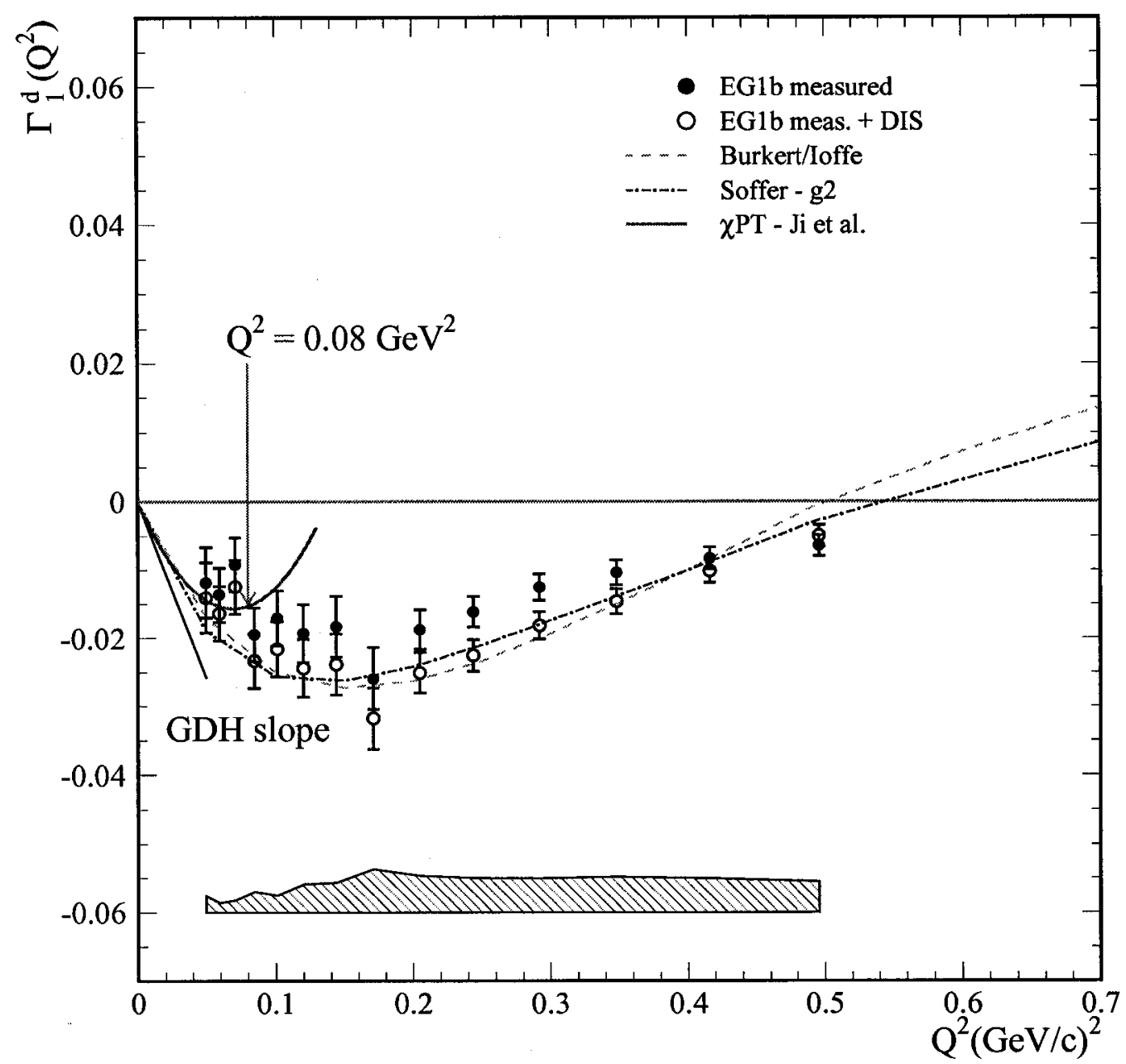

FIG. $120 . \Gamma_{1}$ in the very low $Q^{2}$ region. The error bars show statistical uncertainties. The shaded region at the bottom of the plot indicates the systematic uncertainties for the full integral (EG1b meas. + DIS).

where the leading twist term can be calculated in perturbative QCD. Fig. 121 shows $\Gamma_{1}$ after adding the elastic contribution calculated using the elastic form factors. To calculate the form factors, the Bosted parameterization, which was modified to fit the Jlab data, was used (explained in the section 5.7.5). The twist two term shown as the solid line in Fig. 121 with the uncertainty given by the dashed lines was calculated using the parameterization given in [36] by using the singlet axial charge, $\Delta \Sigma=0.2$ [83]. The renormalization group invariant definition of $\Delta \Sigma$, in the $\overline{M S}$ scheme, with four active quark flavors was used [83] in the calculation. Clearly the higher twist effects are small above $Q^{2} \approx 1.5 \mathrm{GeV}^{2}$, but the effects become increasingly large at 
lower $Q^{2}$ values. As previously shown (fig. 116) duality holds in the second and the third resonance region for $g_{1}$ down to $Q^{2}$ values as low as $0.8 \mathrm{GeV}^{2}$. Therefore, most of the higher twist effects are due to the contributions of the elastic and the delta

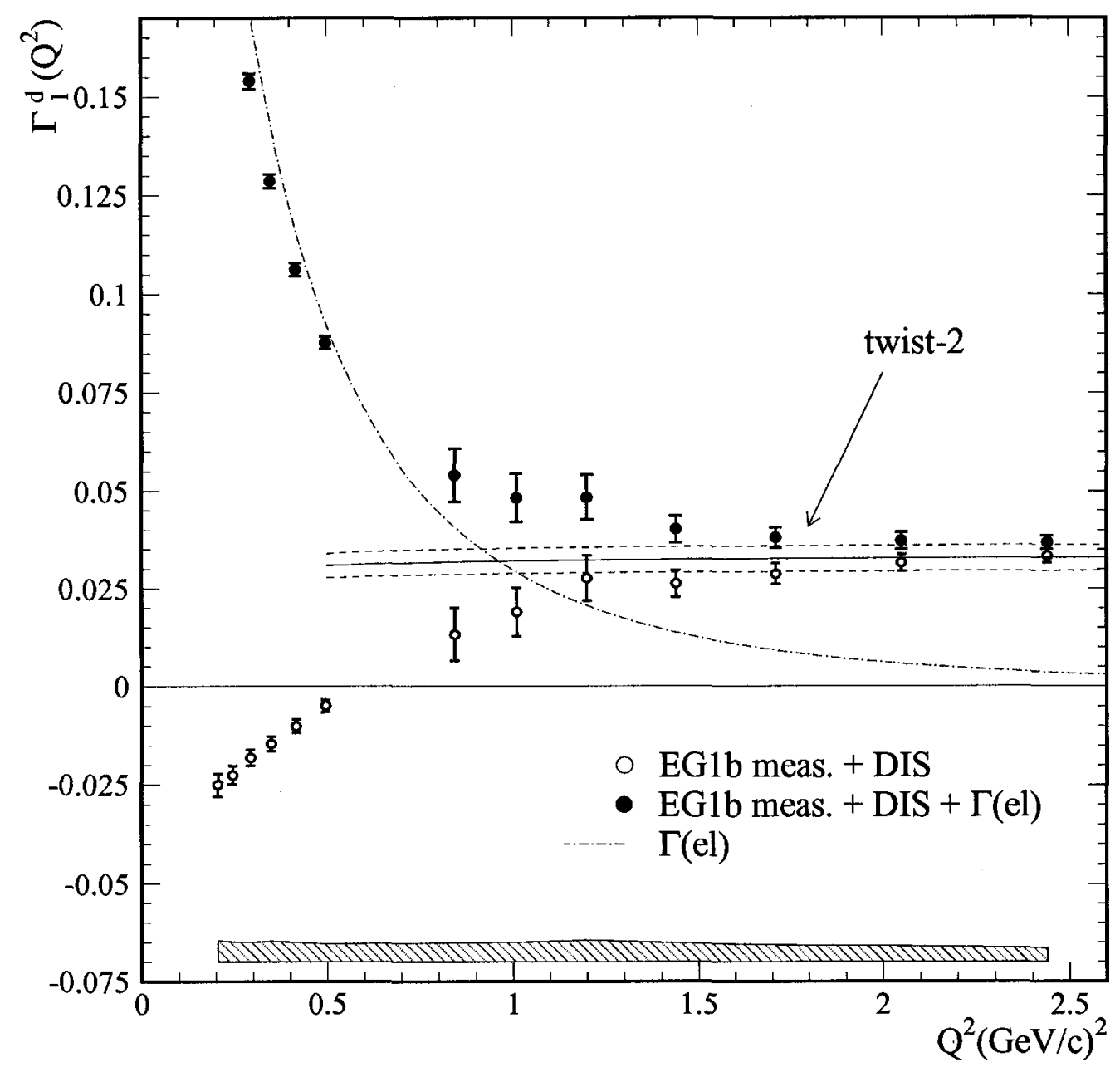

FIG. 121. $\Gamma_{1}$ in the high $Q^{2}$ region. The two dashed lines are $10 \%$ error on $\Gamma_{1}$, which was calculated as described in the text. The error bars show statistical uncertainties. The shaded region at the bottom of the plot indicates the systematic uncertainties for the full integral (EG1b meas. + DIS).

resonance. The large uncertainty on the data for $Q^{2}$ between $0.6-1.3 \mathrm{GeV}^{2}$ will be significantly improved once the remaining EG1 data (2.x and 4.x GeV) are analyzed. These data will be very useful in determining the higher twist contributions to the first moment of $\Gamma_{1}$, which will improve our understanding of the twist-four matrix elements of the nucleon. 
It is evident from the data that the first moment of $g_{1}$ shows a strong $Q^{2}$ dependence. $\mathrm{X}$. Ji points out that this strong $Q^{2}$ dependence has a simple physical interpretation [84]. He argues that the Compton amplitude, which remains in the forward direction, is very much like a diffraction process and $\Gamma_{1}$ can be be viewed as the "brightness" of the diffraction center. The scattering is coherent for low $Q^{2}$ photons giving a large diffraction peak at the center. For somewhat large $Q^{2}$ values, where large fluctuations in the nucleon can be seen, the scattering becomes less coherent. At very large $Q^{2}$ values, since the virtual photon can see the individual quarks, the scattering becomes completely incoherent and the diffraction peak is given by the sum of the diffraction peaks for these individual quarks. Therefore in general the $Q^{2}$ dependence of $\Gamma_{1}$ can be viewed as how the diffraction amplitude of the virtual photon is changed when the virtual mass of the photon, $Q^{2}$, is changed.

From our data many interesting aspects of the spin structure function $g_{1}$ have been understood. The data will play an important role in confirming or disproving existing and future theoretical arguments about the spin structure of the nucleon in the low to moderate $Q^{2}$ region. A more comprehensive summary of what has been learned from these data will be discussed in the next chapter. 


\section{CHAPTER 7 \\ CONCLUSIONS}

The data discussed in this thesis cover a large kinematic region and complement most of the existing world data on the spin structure function $g_{1}$ for the deuteron. At lower $Q^{2}$ values $g_{1}$ shows a considerable structure which makes the first moment, $\Gamma_{1}$, strongly $Q^{2}$ dependent. For $Q^{2}$ values below $\approx 0.55 \mathrm{GeV}^{2}$ the $\Delta$ resonance dominates the whole integral, which makes it negative and results in a minimum around $Q^{2} \approx 0.15 \mathrm{GeV}^{2}$. For $Q^{2}$ values below the minimum the integral becomes less negative and approaches the GDH slope. The data also agree with chiral perturbation theory calculations [37] at next to leading order for momenta below $Q^{2} \approx 0.08 \mathrm{GeV}^{2}$.

At higher $Q^{2}$ values the resonance structure becomes less prominent and the integral is less $Q^{2}$ dependent. For $Q^{2}>2 \mathrm{GeV}^{2}$ higher twist effects are negligible and the data agree with the twist- 2 calculations. For $Q^{2}$ below $2 \mathrm{GeV}^{2}$, higher twist effects become increasingly large. These data, combined with the $2 . x$ and $4 . x \mathrm{GeV}$ data, will be very helpful in evaluating higher twist terms.

The agreement between the data and theoretical calculations at high and low $Q^{2}$ confirms that the first moment of $g_{1}$ can be constructed from the Compton amplitude $S_{1}\left(0, Q^{2}\right)[7]$. We hope that the transition region between parton and hadron degrees of freedom can be compared with lattice QCD calculations in the future.

Although the main goal of the experiment was to calculate the first moment of $g_{1}$, some other important aspects of spin physics were also studied.

The data confirm that the $Q^{2}$ evolution of $g_{1}$ is very similar to the $Q^{2}$ evolution of the unpolarized structure function $F_{1}$ in the lower limit of the DIS region, as has been observed previously in the high $Q^{2}$ region. It was also observed that the resonance structure causes the two structure functions to evolve very differently in the low $Q^{2}$ region.

The virtual photon asymmetry $A_{1}$ at large $x$ provides valuable information about the valence structure of the nucleon. The data show a trend towards unity as $x$ approaches 1 , as predicted by $\mathrm{pQCD}$, implying that the struck quark carries the spin of the entire nucleon as well as the momentum. However, more data in the large $x$ region is required to make precise conclusions. The data agree with the hyperfine perturbed quark model for $x$ above $\approx 0.45$, which suggests that the $\mathrm{SU}(6)$ symmetry is broken due to hyperfine interactions between quarks. 
The wide kinematic coverage of $g_{1}$ also allows us to investigate quark hadron duality. Duality was observed to hold in the resonance region above $Q^{2} \approx 1.4 \mathrm{GeV}^{2}$. However in restricted $\xi$ ranges around the resonance regions $\mathrm{S}_{11}(1535)$ and $\mathrm{F}_{15}(1680)$, local duality was observed for $Q^{2}$ values down to $0.8 \mathrm{GeV}^{2}$, where the higher twist effects are expected to be large.

Our study of the spin structure of the deuteron has provided a wealth of data, which shed light on the interesting transition region between hadron and parton degrees of freedom. 


\section{BIBLIOGRAPHY}

[1] J.Ashman et al., Phys. Lett. B 206, 364 (1988); J.Ashman et al., Nucl. Phys. B 328, 1 (1989).

[2] J. D. Bjorken, Phys. Rev. 179, 1547 (1969).

[3] K. Abe et al., Phys. Rev. D 58, 112003 (1998).

[4] S. B. Gerasimov, Sov. J. Nucl. Phys. 2, 430 (1966); S. D. Drell and A. C. Hearn, Phys. Rev. Lett. 16, 908 (1966).

[5] M. Anselmino, B. L. Ioffe and E. Leader, Sov. J. Nucl. Phys. 49, 136 (1989).

[6] V. D. Burkert and B. L. Ioffe, Phys. Lett. B 296, (1992).

[7] X. Ji and J. Osborne, J. Phys. G 27, 127 (2001).

[8] S. Kuhn et al., CEBAF proposal E-93-009.

[9] M. Anselmino, A. Efremov and E. Leader, Phys. Rept. 261, 1 (1995).

[10] W. Melnitchouk, hep-ph/0006170 (2000).

[11] T. Pussieux and R. Windmolders, A collection of formulas for spin dependent deep inelastic scattering (unpublished).

[12] L. W. Whitlow et al., Phys. Lett. B 250, 193 (1990).

[13] G. Mitchell, in proccedings of the American Physical Society Meeting of the Division of Particles and Fields, 1999, UCLA (hep-ex/9903055).

[14] B. W. Filippone and X. Ji, Adv. Nucl. Phys. 26, 1 (2001).

[15] V. N. Gribov and L. N. Lipatov, Sov. J. Nucl. Phys. 15, 138 (1972); Y. L. Dokahitzer, Sov. Phys. JETP. 16, 161 (1977); G. Altarelli and G. Parisi, Nucl. Phys. B 126, 298 (1977).

[16] R. G Roberts, The structure of the proton (Cambridge University Press, Cambridge, 1990). 
[17] R. Mertig and W. L. VanNeerven, Z Phys. C 70, 637 (1996); W.

Vogelsang, Nucl. Phys. B 475, 47 (1996).

[18] K. Wilson, Phys. Rev. 179, 1499 (1969); R. A. Brandt and G. Preparata, Nucl. Phys. B 27, 541 (1971); O. Nachtmann, Nucl. phys. B 63, 237 (1973).

[19] X. Ji and P. Unrau, Phys. Rev. D 52, 72 (1995).

[20] K. Joo et al., Phys. Rev. Lett. 88, 122001 (2002).

[21] C. E. Carlson and N. C. Mukhopadhyay, Phys. Rev. D 58, 094029 (1998).

[22] T. Gehrmann and W. J. Stirling, Phys. Rev. D 53, 6100 (1996); J. Edelmann, G. Piller, N. kaiser and W. Weise, Nucl. Phys. A 665, 125 (2000).

[23] R. H. Fatemi, Ph.D. Thesis, (2002).

[24] P. L. Anthony et al., Phys. Lett. B 458, 529 (1999); P. L. Anthony et al., Phys. Lett. B 553, 18 (2003).

[25] S. Wandzura and F. Wilczek, Phys Lett. B 172, 195 (1977).

[26] E. D. Bloom and F. J. Gilman, Phys. Rev. lett. 25, 1140 (1970).

[27] I. Niculescu et al., Phys. Rev. Lett. 85, 1186 (2000).

[28] A. D. Rujula, H. Georgi and H. D. Politzer, Ann. Phys. 103, 315 (1977).

[29] C. E. Carlson and N. C. Mukhopadhyay, Phys. Rev. D 41, R2343 (1990).

[30] J. Pumplin et al., JHEP 0207, 012 (2002).

[31] F. E. Close, An Introduction to quarks and partons (Academic Press, London, 1979).

[32] N. Isgur, Phys. Rev. D 59, 34013 (1999).

[33] N. Isgure, G. Krl and R. Koniuk, Phys. Rev. Lett. 41, 1269 (1978); N. Isgure, G. Krl and R. Koniuk, Phys. Rev. Lett. 45, 1738 (1980).

[34] F. E. Close and W. Melnitchouk, Phys. Rev. C 68, 035210 (2003). 
[35] G. R. Farrar and D. R. Jackson, Phys. Rev. Lett. 35, 1416 (1975); G. R. Farrar, Phys. Lett. B 70, 346 (1977).

[36] X. Ji and W. Melnitchouk, Phys. Rev D. 56, 1 (1997).

[37] X. Ji, C. Kao and J. Osborne, Phys. Lett. B 472, 1 (2000).

[38] J. Soffer and O. Teryaev, Phys. Rev. Lett. 70, 3373 (1993); J. Soffer and O. Teryaev, Phys. Rev. D 51, 25 (1995); J. Soffer and O. Teryaev, Phys. Rev. D 56, 7458 (1997).

[39] J. J. Aubert et al., Phys. Lett. B 123, 275 (1983).

[40] J. Yun et al., Phys. Rev. C 67, 055204 (2003).

[41] B. Mecking et al., Nucl. Instr. and Meth. A 503, 513 (2003).

[42] M. D. Mestayer et al., Nucl. Instr. and Meth. A 449, 81 (2000).

[43] E. S. Smith et al., Nucl. Instr. and Meth. A 432, 265 (1999).

[44] G. Adams et al., Nucl. Instr. and Meth. A 465, 414 (2001).

[45] M. Amarian et al., Nucl. Instr. and Meth. A 460, 239 (2001).

[46] C. D. Keith et al., Nucl. Instr. and Meth. A 501, 327 (2003).

[47] M. Goldman, J. Magn. Res. 17, 393 (1975).

[48] http://www.jlab.org/Hall-B/ ${ }^{\sim}$ shifeng.

[49] E. Smith et al., CLAS-NOTE 1999-011.

[50] A. Vlassov et al., CLAS-NOTE 1990-026.

[51] D. Lawrence et al., CLAS-NOTE 1999-018.

[52] R. De Vita, Private communications.

[53] A. Vlassov, http://www.jlab.org/ vlassov/cc/index.html.

[54] P. Bosted et al., CLAS NOTE 2003-008.

[55] A. Klimenko and S. Kuhn, CLAS NOTE 2003-005. 
[56] S. E. Kuhn, http://www.jlab.org/Hall-

B/secure/eg1/AnalysisDoc/EG2000DataAnalysis.pdf.

[57] P. Bosted, Private communications.

[58] P. Bosted, CLAS-NOTE 2004-005.

[59] J. Yun and S. Kuhn, http://www.jlab.org/Hall-B/secure/eg1/ND3_analysis/ana_note.pdf.

[60] T. W. Donnelly and A. S. Raskin, Annals of Phys. 191, 78 (1989).

[61] P. E. Bosted, Phys. Rev. C 51, 409 (1995).

[62] S. E. Kuhn, Private communications.

[63] Tingjun Liu, Ph.D thesis; T. Averett, E155 Technical Note 27; K. Abe et al., Phys. Rev. D 58, 112003 (1998).

[64] Y. S. Tsai, Report No. SLAC_PUB-848 (1971); Y. S. Tsai, Rev. Mod. Phys. 46, 815 (1974).

[65] H. Olsen and L. C. Maximon, Phys. Rev. 114, 887 (1959).

[66] T. V. Kuchto and N. M. Shumeiko, Nucl. Phys. B 219, 412 (1983).

[67] F. R. Wesselmann and S. E. Kuhn E155 Tech. Note No. 100 (1999); F. R. Wesselmann and S. E. Kuhn E155 Tech. Note No. 59 (1998).

[68] P. L. Anthony et al., Phys. Lett. B 493, 19 (2000).

[69] D. Drechsel et al., Nucl. Phys. A 645, 145 (1999).

[70] R. L. Walker, Phys. Rev. 182, 1729 (1969).

[71] M. Arneodo et al., Phys. Lett. B 364, 108 (1995).

[72] K. Abe et al., Phys. Lett. B 452, 194 (1999).

[73] S. S. Kamalov et al., Phys. Rev. Lett. 83, 22 (1999).

[74] J. Soffer and O. V. Teryaev, Phys. Lett. B 490, 106 (2000).

[75] L. W. Whitlow, SLAC-Report-357 (1990). 
[76] G. Ricco et al., Nucl. Phys. B 555, 306 (1999).

[77] A. Bodek et al., Phys. Rev. D 20, 1471 (1979).

[78] A. Bodek et al., Phys. Rev. Lett. 30, 1087 (1973); E. M. Riordan et al., Phys. Lett. B 52, 249 (1974); A. Bodek et al., Phys. Lett. B 51, 417 (1974); J. S. Poucher, Ph.D. thesis (1771).

[79] C. E. Keppel, in proceedings of the SPIN 2002 conforence, 2002, New York.

[80] M. Glueck, E. Reya, M. Stratmann and W. Vogelsang, Phys. Rev. D 63, 094005 (2001).

[81] X. Zheng et al., Phys. Rev. Lett. 92, 012004 (2004).

[82] X. Ji and J. Osborne, hep-ph/9905410.

[83] W. Melnitchouk, Private communications.

[84] X. Ji, in proceedings of the NSTAR 2000 conference, 2000, Virginia. 
APPENDIX A

DATA TABLES FOR $g_{1}(\mathbf{E}=1 . x \mathrm{GeV})$ 
TABLE XIII. Results for $g_{1}$ for $Q^{2}=0.05 \mathrm{GeV}^{2}$.

\begin{tabular}{|c|c|}
\hline$x$ & $g_{1} \pm$ stat \pm syst \\
\hline 0.1393 & $-0.3377 \pm 0.2426 \pm 0.2265$ \\
0.1115 & $-0.0223 \pm 0.2333 \pm 0.0173$ \\
0.0924 & $-0.2486 \pm 0.2546 \pm 0.0447$ \\
0.0785 & $-0.5482 \pm 0.2608 \pm 0.0662$ \\
0.0679 & $0.0413 \pm 0.2606 \pm 0.0194$ \\
0.0596 & $-0.3544 \pm 0.2464 \pm 0.0367$ \\
0.0529 & $-0.0152 \pm 0.2080 \pm 0.0155$ \\
0.0475 & $-0.2723 \pm 0.1877 \pm 0.0330$ \\
0.0429 & $-0.3897 \pm 0.1828 \pm 0.0497$ \\
0.0390 & $-0.0366 \pm 0.1880 \pm 0.0128$ \\
0.0357 & $-0.4738 \pm 0.2039 \pm 0.0442$ \\
0.0329 & $-0.0736 \pm 0.2157 \pm 0.0146$ \\
0.0304 & $-0.2016 \pm 0.2134 \pm 0.0213$ \\
\hline
\end{tabular}

TABLE XIV. Results for $g_{1}$ for $Q^{2}=0.06 \mathrm{GeV}^{2}$.

\begin{tabular}{|c|c|}
\hline$x$ & $g_{1} \pm$ stat \pm syst \\
\hline 0.1619 & $0.0511 \pm 0.1407 \pm 0.0415$ \\
0.1303 & $-0.0591 \pm 0.1526 \pm 0.0280$ \\
0.1083 & $-0.3308 \pm 0.1860 \pm 0.0680$ \\
0.0923 & $-0.6648 \pm 0.2111 \pm 0.0846$ \\
0.0800 & $-0.6530 \pm 0.2060 \pm 0.0623$ \\
0.0703 & $-0.3494 \pm 0.1896 \pm 0.0392$ \\
0.0626 & $-0.2161 \pm 0.1754 \pm 0.0257$ \\
0.0561 & $-0.2501 \pm 0.1665 \pm 0.0307$ \\
0.0508 & $-0.1151 \pm 0.1616 \pm 0.0177$ \\
0.0463 & $-0.3845 \pm 0.1729 \pm 0.0460$ \\
0.0424 & $0.0398 \pm 0.1866 \pm 0.0152$ \\
0.0390 & $-0.0930 \pm 0.1998 \pm 0.0144$ \\
0.0360 & $0.0426 \pm 0.2035 \pm 0.0152$ \\
\hline
\end{tabular}


TABLE XV. Results for $g_{1}$ for $Q^{2}=0.07 \mathrm{GeV}^{2}$.

\begin{tabular}{|c|c|}
\hline$x$ & $g_{1} \pm$ stat \pm syst \\
\hline 0.1875 & $-0.0013 \pm 0.1140 \pm 0.0193$ \\
0.1518 & $0.1168 \pm 0.1341 \pm 0.0550$ \\
0.1267 & $-0.3435 \pm 0.1627 \pm 0.0794$ \\
0.1083 & $-0.7149 \pm 0.1794 \pm 0.0951$ \\
0.0941 & $-0.3691 \pm 0.1828 \pm 0.0425$ \\
0.0829 & $-0.0200 \pm 0.1773 \pm 0.0199$ \\
0.0738 & $0.0314 \pm 0.1633 \pm 0.0198$ \\
0.0663 & $-0.0339 \pm 0.1497 \pm 0.0183$ \\
0.0601 & $-0.1237 \pm 0.1483 \pm 0.0212$ \\
0.0547 & $-0.1815 \pm 0.1583 \pm 0.0227$ \\
0.0502 & $-0.4828 \pm 0.1756 \pm 0.0422$ \\
0.0462 & $0.1146 \pm 0.1901 \pm 0.0204$ \\
0.0427 & $-0.0467 \pm 0.1950 \pm 0.0162$ \\
\hline
\end{tabular}

TABLE XVI. Results for $g_{1}$ for $Q^{2}=0.084 \mathrm{GeV}^{2}$.

\begin{tabular}{|c|c|}
\hline$x$ & $g_{1} \pm$ stat \pm syst \\
\hline 0.2160 & $-0.0518 \pm 0.0985 \pm 0.0359$ \\
0.1760 & $0.0227 \pm 0.1166 \pm 0.0241$ \\
0.1477 & $-0.1742 \pm 0.1439 \pm 0.0538$ \\
0.1266 & $-0.8211 \pm 0.1646 \pm 0.1126$ \\
0.1103 & $-0.6367 \pm 0.1660 \pm 0.0716$ \\
0.0974 & $-0.2793 \pm 0.1579 \pm 0.0373$ \\
0.0869 & $-0.0288 \pm 0.1499 \pm 0.0218$ \\
0.0782 & $-0.0923 \pm 0.1403 \pm 0.0207$ \\
0.0709 & $0.0216 \pm 0.1363 \pm 0.0158$ \\
0.0647 & $-0.1653 \pm 0.1465 \pm 0.0221$ \\
0.0593 & $-0.0287 \pm 0.1649 \pm 0.0154$ \\
0.0547 & $-0.3408 \pm 0.1766 \pm 0.0266$ \\
0.0506 & $-0.2333 \pm 0.1790 \pm 0.0244$ \\
\hline
\end{tabular}


TABLE XVII. Results for $g_{1}$ for $Q^{2}=0.1 \mathrm{GeV}^{2}$.

\begin{tabular}{|c|c|}
\hline$x$ & $g_{1} \pm$ stat \pm syst \\
\hline 0.2479 & $0.1253 \pm 0.0861 \pm 0.0916$ \\
0.2036 & $-0.1800 \pm 0.1041 \pm 0.0750$ \\
0.1717 & $-0.2358 \pm 0.1313 \pm 0.0630$ \\
0.1478 & $-0.5164 \pm 0.1511 \pm 0.0798$ \\
0.1292 & $-0.4565 \pm 0.1531 \pm 0.0580$ \\
0.1143 & $-0.4115 \pm 0.1463 \pm 0.0540$ \\
0.1022 & $-0.0315 \pm 0.1382 \pm 0.0238$ \\
0.0921 & $-0.1991 \pm 0.1288 \pm 0.0292$ \\
0.0837 & $-0.0089 \pm 0.1289 \pm 0.0188$ \\
0.0764 & $-0.0522 \pm 0.1366 \pm 0.0160$ \\
0.0702 & $-0.0464 \pm 0.1522 \pm 0.0169$ \\
0.0647 & $-0.1951 \pm 0.1611 \pm 0.0222$ \\
0.0600 & $0.0257 \pm 0.1659 \pm 0.0204$ \\
\hline
\end{tabular}

TABLE XVIII. Results for $g_{1}$ for $Q^{2}=0.12 \mathrm{GeV}^{2}$.

\begin{tabular}{|c|c|}
\hline$x$ & $g_{1} \pm$ stat \pm syst \\
\hline 0.2814 & $-0.0190 \pm 0.0778 \pm 0.0246$ \\
0.2329 & $-0.1184 \pm 0.0961 \pm 0.0562$ \\
0.1976 & $-0.2019 \pm 0.1243 \pm 0.0525$ \\
0.1708 & $-0.2795 \pm 0.1447 \pm 0.0452$ \\
0.1499 & $-0.2784 \pm 0.1468 \pm 0.0418$ \\
0.1330 & $-0.5273 \pm 0.1408 \pm 0.0712$ \\
0.1191 & $-0.2334 \pm 0.1322 \pm 0.0354$ \\
0.1076 & $-0.1582 \pm 0.1231 \pm 0.0278$ \\
0.0978 & $-0.1588 \pm 0.1214 \pm 0.0250$ \\
0.0895 & $-0.2235 \pm 0.1261 \pm 0.0281$ \\
0.0823 & $-0.0002 \pm 0.1419 \pm 0.0179$ \\
0.0760 & $0.1192 \pm 0.1530 \pm 0.0229$ \\
0.0705 & $0.1629 \pm 0.1613 \pm 0.0249$ \\
\hline
\end{tabular}


TABLE XIX. Results for $g_{1}$ for $Q^{2}=0.14 \mathrm{GeV}^{2}$.

\begin{tabular}{|c|c|}
\hline$x$ & $g_{1} \pm$ stat \pm syst \\
\hline 0.3197 & $-0.1349 \pm 0.0713 \pm 0.0911$ \\
0.2671 & $-0.0481 \pm 0.0918 \pm 0.0299$ \\
0.2281 & $-0.1813 \pm 0.1199 \pm 0.0520$ \\
0.1982 & $-0.5860 \pm 0.1383 \pm 0.1043$ \\
0.1746 & $-0.4686 \pm 0.1385 \pm 0.0694$ \\
0.1554 & $-0.1220 \pm 0.1304 \pm 0.0264$ \\
0.1396 & $0.0476 \pm 0.1216 \pm 0.0254$ \\
0.1264 & $-0.1451 \pm 0.1150 \pm 0.0278$ \\
0.1152 & $-0.0336 \pm 0.1154 \pm 0.0207$ \\
0.1055 & $-0.0792 \pm 0.1211 \pm 0.0210$ \\
0.0971 & $0.1360 \pm 0.1353 \pm 0.0227$ \\
0.0898 & $-0.0404 \pm 0.1459 \pm 0.0223$ \\
0.0834 & $0.3315 \pm 0.1480 \pm 0.0352$ \\
\hline
\end{tabular}

TABLE XX. Results for $g_{1}$ for $Q^{2}=0.17 \mathrm{GeV}^{2}$.

\begin{tabular}{|c|c|}
\hline$x$ & $g_{1} \pm$ stat \pm syst \\
\hline 0.3582 & $-0.0435 \pm 0.0635 \pm 0.0343$ \\
0.3020 & $-0.1908 \pm 0.0829 \pm 0.0855$ \\
0.2598 & $-0.2511 \pm 0.1077 \pm 0.0724$ \\
0.2270 & $-0.4591 \pm 0.1264 \pm 0.0841$ \\
0.2008 & $-0.8253 \pm 0.1275 \pm 0.1301$ \\
0.1794 & $-0.0942 \pm 0.1210 \pm 0.0253$ \\
0.1616 & $-0.0571 \pm 0.1138 \pm 0.0235$ \\
0.1466 & $0.0526 \pm 0.1094 \pm 0.0244$ \\
0.1339 & $0.0621 \pm 0.1080 \pm 0.0229$ \\
0.1229 & $0.0629 \pm 0.1154 \pm 0.0223$ \\
0.1133 & $0.1335 \pm 0.1273 \pm 0.0245$ \\
0.1049 & $-0.1612 \pm 0.1298 \pm 0.0266$ \\
0.0975 & $0.0438 \pm 0.1313 \pm 0.0274$ \\
\hline
\end{tabular}


TABLE XXI. Results for $g_{1}$ for $Q^{2}=0.2 \mathrm{GeV}^{2}$.

\begin{tabular}{|c|c|}
\hline$x$ & $g_{1} \pm$ stat \pm syst \\
\hline 0.4009 & $-0.0087 \pm 0.0413 \pm 0.0227$ \\
0.3416 & $0.0024 \pm 0.0520 \pm 0.0227$ \\
0.2962 & $-0.1873 \pm 0.0636 \pm 0.0519$ \\
0.2604 & $-0.4413 \pm 0.0709 \pm 0.0861$ \\
0.2314 & $-0.4307 \pm 0.0707 \pm 0.0714$ \\
0.2076 & $-0.1872 \pm 0.0647 \pm 0.0353$ \\
0.1877 & $-0.0480 \pm 0.0597 \pm 0.0240$ \\
0.1708 & $0.0170 \pm 0.0534 \pm 0.0243$ \\
0.1563 & $0.0050 \pm 0.0500 \pm 0.0234$ \\
0.1438 & $-0.0533 \pm 0.0519 \pm 0.0248$ \\
0.1328 & $0.0628 \pm 0.0546 \pm 0.0247$ \\
0.1232 & $0.0875 \pm 0.0569 \pm 0.0285$ \\
0.1146 & $-0.0146 \pm 0.0597 \pm 0.0310$ \\
0.1070 & $0.1814 \pm 0.0640 \pm 0.0313$ \\
0.1002 & $0.0383 \pm 0.0686 \pm 0.0300$ \\
0.0941 & $-0.0238 \pm 0.0713 \pm 0.0317$ \\
0.0885 & $-0.1162 \pm 0.0904 \pm 0.0352$ \\
\hline
\end{tabular}


TABLE XXII. Results for $g_{1}$ for $Q^{2}=0.24 \mathrm{GeV}^{2}$.

\begin{tabular}{|c|c|}
\hline$x$ & $g_{1} \pm$ stat \pm syst \\
\hline 0.4433 & $-0.0024 \pm 0.0290 \pm 0.0216$ \\
0.3817 & $0.0037 \pm 0.0366 \pm 0.0218$ \\
0.3337 & $-0.1859 \pm 0.0474 \pm 0.0523$ \\
0.2953 & $-0.3659 \pm 0.0533 \pm 0.0712$ \\
0.2638 & $-0.3834 \pm 0.0517 \pm 0.0633$ \\
0.2377 & $-0.2012 \pm 0.0475 \pm 0.0363$ \\
0.2157 & $-0.1420 \pm 0.0439 \pm 0.0281$ \\
0.1969 & $0.0626 \pm 0.0420 \pm 0.0241$ \\
0.1807 & $0.0498 \pm 0.0413 \pm 0.0239$ \\
0.1666 & $0.0313 \pm 0.0433 \pm 0.0265$ \\
0.1542 & $0.0369 \pm 0.0483 \pm 0.0265$ \\
0.1433 & $0.1401 \pm 0.0501 \pm 0.0323$ \\
0.1335 & $0.0465 \pm 0.0497 \pm 0.0343$ \\
0.1248 & $0.0599 \pm 0.0545 \pm 0.0319$ \\
0.1170 & $0.0678 \pm 0.0568 \pm 0.0311$ \\
0.1100 & $-0.0022 \pm 0.0610 \pm 0.0324$ \\
0.1036 & $-0.0623 \pm 0.0759 \pm 0.0355$ \\
\hline
\end{tabular}


TABLE XXIII. Results for $g_{1}$ for $Q^{2}=0.3 \mathrm{GeV}^{2}$.

\begin{tabular}{|c|c|}
\hline$x$ & $g_{1} \pm$ stat \pm syst \\
\hline 0.4880 & $0.0157 \pm 0.0218 \pm 0.0236$ \\
0.4249 & $-0.0151 \pm 0.0290 \pm 0.0218$ \\
0.3748 & $-0.1421 \pm 0.0372 \pm 0.0418$ \\
0.3340 & $-0.2765 \pm 0.0425 \pm 0.0525$ \\
0.3002 & $-0.3307 \pm 0.0436 \pm 0.0521$ \\
0.2718 & $-0.1645 \pm 0.0410 \pm 0.0290$ \\
0.2476 & $-0.0494 \pm 0.0383 \pm 0.0222$ \\
0.2269 & $-0.0191 \pm 0.0370 \pm 0.0217$ \\
0.2088 & $0.0054 \pm 0.0367 \pm 0.0227$ \\
0.1930 & $0.0931 \pm 0.0385 \pm 0.0283$ \\
0.1791 & $0.0828 \pm 0.0426 \pm 0.0285$ \\
0.1667 & $0.2251 \pm 0.0442 \pm 0.0358$ \\
0.1557 & $0.0789 \pm 0.0447 \pm 0.0370$ \\
0.1458 & $0.0786 \pm 0.0469 \pm 0.0337$ \\
0.1369 & $0.1076 \pm 0.0523 \pm 0.0321$ \\
0.1288 & $-0.0003 \pm 0.0559 \pm 0.0331$ \\
\hline
\end{tabular}

TABLE XXIV. Results for $g_{1}$ for $Q^{2}=0.35 \mathrm{GeV}^{2}$.

\begin{tabular}{|c|c|}
\hline$x$ & $g_{1} \pm$ stat \pm syst \\
\hline 0.5318 & $-0.0108 \pm 0.0191 \pm 0.0205$ \\
0.4683 & $-0.0303 \pm 0.0255 \pm 0.0219$ \\
0.4167 & $-0.1156 \pm 0.0327 \pm 0.0322$ \\
0.3740 & $-0.3461 \pm 0.0375 \pm 0.0609$ \\
0.3383 & $-0.2837 \pm 0.0376 \pm 0.0422$ \\
0.3079 & $-0.1403 \pm 0.0356 \pm 0.0249$ \\
0.2817 & $-0.0187 \pm 0.0334 \pm 0.0203$ \\
0.2591 & $0.0957 \pm 0.0321 \pm 0.0214$ \\
0.2393 & $0.0759 \pm 0.0320 \pm 0.0223$ \\
0.2218 & $0.0744 \pm 0.0342 \pm 0.0276$ \\
0.2064 & $0.1579 \pm 0.0386 \pm 0.0299$ \\
0.1926 & $0.1448 \pm 0.0412 \pm 0.0358$ \\
0.1802 & $0.1907 \pm 0.0432 \pm 0.0390$ \\
0.1691 & $0.1370 \pm 0.0443 \pm 0.0350$ \\
0.1590 & $0.1566 \pm 0.0481 \pm 0.0328$ \\
\hline
\end{tabular}


TABLE XXV. Results for $g_{1}$ for $Q^{2}=0.42 \mathrm{GeV}^{2}$.

\begin{tabular}{|c|c|}
\hline$x$ & $g_{1} \pm$ stat \pm syst \\
\hline 0.5759 & $-0.0125 \pm 0.0168 \pm 0.0195$ \\
0.5128 & $-0.0259 \pm 0.0219 \pm 0.0198$ \\
0.4606 & $-0.1310 \pm 0.0277 \pm 0.0312$ \\
0.4167 & $-0.2110 \pm 0.0319 \pm 0.0346$ \\
0.3793 & $-0.2970 \pm 0.0320 \pm 0.0384$ \\
0.3471 & $-0.1115 \pm 0.0302 \pm 0.0202$ \\
0.3192 & $-0.0113 \pm 0.0284 \pm 0.0184$ \\
0.2948 & $0.0510 \pm 0.0279 \pm 0.0183$ \\
0.2733 & $0.0705 \pm 0.0293 \pm 0.0204$ \\
0.2542 & $0.1082 \pm 0.0328 \pm 0.0262$ \\
0.2371 & $0.2226 \pm 0.0377 \pm 0.0305$ \\
0.2218 & $0.2111 \pm 0.0414 \pm 0.0373$ \\
0.2081 & $0.1331 \pm 0.0414 \pm 0.0386$ \\
0.1956 & $0.1944 \pm 0.0438 \pm 0.0358$ \\
\hline
\end{tabular}

TABLE XXVI. Results for $g_{1}$ for $Q^{2}=0.5 \mathrm{GeV}^{2}$.

\begin{tabular}{|c|c|}
\hline$x$ & $g_{1} \pm$ stat \pm syst \\
\hline 0.6182 & $-0.0426 \pm 0.0155 \pm 0.0312$ \\
0.5566 & $-0.0049 \pm 0.0201 \pm 0.0145$ \\
0.5045 & $-0.1236 \pm 0.0253 \pm 0.0247$ \\
0.4599 & $-0.1430 \pm 0.0293 \pm 0.0190$ \\
0.4215 & $-0.1719 \pm 0.0294 \pm 0.0182$ \\
0.3880 & $-0.1535 \pm 0.0278 \pm 0.0181$ \\
0.3586 & $-0.0141 \pm 0.0270 \pm 0.0163$ \\
0.3326 & $0.0434 \pm 0.0265 \pm 0.0172$ \\
0.3095 & $0.0088 \pm 0.0288 \pm 0.0182$ \\
0.2889 & $0.0928 \pm 0.0326 \pm 0.0236$ \\
0.2704 & $0.1796 \pm 0.0373 \pm 0.0286$ \\
0.2537 & $0.2169 \pm 0.0415 \pm 0.0353$ \\
0.2386 & $0.1752 \pm 0.0471 \pm 0.0374$ \\
0.2248 & $0.2603 \pm 0.1192 \pm 0.0372$ \\
\hline
\end{tabular}


TABLE XXVII. Results for $g_{1}$ for $Q^{2}=0.6 \mathrm{GeV}^{2}$.

\begin{tabular}{|c|c|}
\hline$x$ & $g_{1} \pm$ stat \pm syst \\
\hline 0.6590 & $-0.0173 \pm 0.0133 \pm 0.0175$ \\
0.5997 & $-0.0196 \pm 0.0183 \pm 0.0138$ \\
0.5486 & $-0.0698 \pm 0.0227 \pm 0.0139$ \\
0.5041 & $-0.1588 \pm 0.0259 \pm 0.0189$ \\
0.4651 & $-0.1683 \pm 0.0266 \pm 0.0153$ \\
0.4307 & $-0.0524 \pm 0.0257 \pm 0.0137$ \\
0.4002 & $-0.0529 \pm 0.0258 \pm 0.0154$ \\
0.3730 & $0.0271 \pm 0.0255 \pm 0.0167$ \\
0.3486 & $0.0511 \pm 0.0282 \pm 0.0186$ \\
0.3266 & $0.1096 \pm 0.0348 \pm 0.0232$ \\
0.3067 & $0.2267 \pm 0.0558 \pm 0.0286$ \\
\hline
\end{tabular}




\section{APPENDIX B}

DATA TABLES FOR $g_{1}(\mathbf{E}=5 . \mathrm{x} \mathrm{GeV})$ 
TABLE XXVIII. Results for $g_{1}$ for $Q^{2}=0.84 \mathrm{GeV}^{2}$.

\begin{tabular}{|c|c|}
\hline$x$ & $g_{1} \pm$ stat \pm syst \\
\hline 0.6937 & $-0.0120 \pm 0.0412 \pm 0.0101$ \\
0.5818 & $0.0133 \pm 0.0666 \pm 0.0115$ \\
0.4951 & $0.0138 \pm 0.0638 \pm 0.0053$ \\
0.4265 & $0.1772 \pm 0.0689 \pm 0.0215$ \\
0.3714 & $0.1741 \pm 0.0819 \pm 0.0224$ \\
0.3263 & $0.1065 \pm 0.0866 \pm 0.0159$ \\
0.2890 & $0.0953 \pm 0.0941 \pm 0.0196$ \\
0.2577 & $0.1334 \pm 0.0960 \pm 0.0213$ \\
0.2313 & $-0.1082 \pm 0.0981 \pm 0.0164$ \\
0.2088 & $-0.1096 \pm 0.0996 \pm 0.0240$ \\
0.1894 & $0.1438 \pm 0.1036 \pm 0.0394$ \\
0.1726 & $0.1135 \pm 0.1067 \pm 0.0227$ \\
0.1579 & $0.0541 \pm 0.1179 \pm 0.0243$ \\
0.1451 & $-0.1044 \pm 0.1225 \pm 0.0229$ \\
0.1337 & $0.1390 \pm 0.1266 \pm 0.0293$ \\
0.1236 & $-0.0596 \pm 0.1347 \pm 0.0240$ \\
0.1147 & $0.0072 \pm 0.1314 \pm 0.0258$ \\
0.1066 & $0.0339 \pm 0.2501 \pm 0.0479$ \\
\hline
\end{tabular}


TABLE XXIX. Results for $g_{1}$ for $Q^{2}=1.0 \mathrm{GeV}^{2}$.

\begin{tabular}{|c|c|}
\hline$x$ & $g_{1} \pm$ stat \pm syst \\
\hline 0.7305 & $-0.0153 \pm 0.0372 \pm 0.0106$ \\
0.6247 & $-0.0922 \pm 0.0587 \pm 0.0088$ \\
0.5399 & $-0.0013 \pm 0.0576 \pm 0.0089$ \\
0.4709 & $0.0922 \pm 0.0633 \pm 0.0122$ \\
0.4142 & $0.1270 \pm 0.0746 \pm 0.0183$ \\
0.3669 & $-0.0330 \pm 0.0794 \pm 0.0101$ \\
0.3272 & $0.3083 \pm 0.0865 \pm 0.0397$ \\
0.2935 & $0.1902 \pm 0.0878 \pm 0.0266$ \\
0.2648 & $0.2395 \pm 0.0915 \pm 0.0319$ \\
0.2400 & $0.0233 \pm 0.0949 \pm 0.0127$ \\
0.2185 & $0.2995 \pm 0.0966 \pm 0.0476$ \\
0.1997 & $0.0810 \pm 0.1021 \pm 0.0197$ \\
0.1833 & $-0.1240 \pm 0.1121 \pm 0.0221$ \\
0.1688 & $0.2384 \pm 0.1168 \pm 0.0383$ \\
0.1559 & $0.2996 \pm 0.1180 \pm 0.0500$ \\
0.1444 & $0.1973 \pm 0.1168 \pm 0.0344$ \\
0.1342 & $0.1744 \pm 0.2197 \pm 0.0372$ \\
\hline
\end{tabular}


TABLE XXX. Results for $g_{1}$ for $Q^{2}=1.2 \mathrm{GeV}^{2}$.

\begin{tabular}{|c|c|}
\hline$x$ & $g_{1} \pm$ stat \pm syst \\
\hline 0.7630 & $0.0638 \pm 0.0344 \pm 0.0414$ \\
0.6642 & $-0.0222 \pm 0.0530 \pm 0.0038$ \\
0.5823 & $0.0712 \pm 0.0527 \pm 0.0100$ \\
0.5140 & $0.0087 \pm 0.0587 \pm 0.0098$ \\
0.4565 & $0.1128 \pm 0.0677 \pm 0.0156$ \\
0.4078 & $0.1312 \pm 0.0727 \pm 0.0193$ \\
0.3662 & $0.1193 \pm 0.0812 \pm 0.0186$ \\
0.3305 & $0.1902 \pm 0.0819 \pm 0.0293$ \\
0.2996 & $0.0324 \pm 0.0849 \pm 0.0207$ \\
0.2728 & $0.2194 \pm 0.0891 \pm 0.0287$ \\
0.2493 & $0.1831 \pm 0.0928 \pm 0.0250$ \\
0.2287 & $0.0208 \pm 0.1038 \pm 0.0157$ \\
0.2105 & $0.2029 \pm 0.1047 \pm 0.0291$ \\
0.1943 & $0.0137 \pm 0.1020 \pm 0.0195$ \\
0.1799 & $0.1775 \pm 0.1049 \pm 0.0294$ \\
0.1671 & $0.1264 \pm 0.1121 \pm 0.0295$ \\
0.1555 & $0.0416 \pm 0.2102 \pm 0.0444$ \\
\hline
\end{tabular}


TABLE XXXI. Results for $g_{1}$ for $Q^{2}=1.4 \mathrm{GeV}^{2}$.

\begin{tabular}{|c|c|}
\hline$x$ & $g_{1} \pm$ stat \pm syst \\
\hline 0.7944 & $-0.0316 \pm 0.0226 \pm 0.0200$ \\
0.7036 & $-0.0205 \pm 0.0348 \pm 0.0060$ \\
0.6259 & $0.0275 \pm 0.0327 \pm 0.0059$ \\
0.5593 & $0.0598 \pm 0.0364 \pm 0.0102$ \\
0.5020 & $0.1264 \pm 0.0402 \pm 0.0157$ \\
0.4524 & $0.0844 \pm 0.0417 \pm 0.0129$ \\
0.4095 & $0.2299 \pm 0.0453 \pm 0.0264$ \\
0.3720 & $0.1309 \pm 0.0472 \pm 0.0171$ \\
0.3392 & $0.1344 \pm 0.0475 \pm 0.0255$ \\
0.3104 & $0.0614 \pm 0.0487 \pm 0.0124$ \\
0.2850 & $0.1193 \pm 0.0520 \pm 0.0150$ \\
0.2625 & $0.1580 \pm 0.0554 \pm 0.0198$ \\
0.2424 & $0.1682 \pm 0.0542 \pm 0.0257$ \\
0.2245 & $0.1092 \pm 0.0544 \pm 0.0219$ \\
0.2084 & $0.1057 \pm 0.0534 \pm 0.0218$ \\
0.1940 & $0.0708 \pm 0.0533 \pm 0.0218$ \\
0.1810 & $0.2993 \pm 0.0959 \pm 0.0402$ \\
\hline
\end{tabular}


TABLE XXXII. Results for $g_{1}$ for $Q^{2}=1.7 \mathrm{GeV}^{2}$.

\begin{tabular}{|c|c|}
\hline$x$ & $g_{1} \pm$ stat \pm syst \\
\hline 0.8211 & $-0.0125 \pm 0.0154 \pm 0.0086$ \\
0.7381 & $-0.0313 \pm 0.0247 \pm 0.0039$ \\
0.6652 & $0.0091 \pm 0.0240 \pm 0.0040$ \\
0.6011 & $0.0344 \pm 0.0281 \pm 0.0070$ \\
0.5448 & $0.0881 \pm 0.0319 \pm 0.0116$ \\
0.4953 & $0.1244 \pm 0.0336 \pm 0.0176$ \\
0.4516 & $0.0898 \pm 0.0366 \pm 0.0119$ \\
0.4130 & $0.1928 \pm 0.0388 \pm 0.0255$ \\
0.3788 & $0.1247 \pm 0.0384 \pm 0.0155$ \\
0.3484 & $0.1590 \pm 0.0401 \pm 0.0173$ \\
0.3213 & $0.1630 \pm 0.0437 \pm 0.0190$ \\
0.2970 & $0.1812 \pm 0.0453 \pm 0.0219$ \\
0.2753 & $0.1681 \pm 0.0451 \pm 0.0227$ \\
0.2558 & $0.0642 \pm 0.0455 \pm 0.0191$ \\
0.2382 & $0.0953 \pm 0.0453 \pm 0.0192$ \\
0.2223 & $0.1158 \pm 0.0860 \pm 0.0288$ \\
\hline
\end{tabular}


TABLE XXXIII. Results for $g_{1}$ for $Q^{2}=2.0 \mathrm{GeV}^{2}$.

\begin{tabular}{|c|c|}
\hline$x$ & $g_{1} \pm$ stat \pm syst \\
\hline 0.8462 & $0.0058 \pm 0.0131 \pm 0.0080$ \\
0.7716 & $0.0071 \pm 0.0197 \pm 0.0029$ \\
0.7043 & $0.0187 \pm 0.0198 \pm 0.0040$ \\
0.6437 & $0.0296 \pm 0.0232 \pm 0.0075$ \\
0.5893 & $0.0723 \pm 0.0260 \pm 0.0102$ \\
0.5405 & $0.0642 \pm 0.0268 \pm 0.0093$ \\
0.4968 & $0.1505 \pm 0.0298 \pm 0.0175$ \\
0.4575 & $0.1657 \pm 0.0319 \pm 0.0199$ \\
0.4223 & $0.0997 \pm 0.0327 \pm 0.0137$ \\
0.3906 & $0.0782 \pm 0.0355 \pm 0.0129$ \\
0.3620 & $0.1062 \pm 0.0371 \pm 0.0140$ \\
0.3362 & $0.1010 \pm 0.0382 \pm 0.0151$ \\
0.3129 & $0.1610 \pm 0.0387 \pm 0.0211$ \\
0.2918 & $0.1000 \pm 0.0397 \pm 0.0172$ \\
0.2727 & $0.1108 \pm 0.0403 \pm 0.0212$ \\
0.2552 & $0.0364 \pm 0.0740 \pm 0.0180$ \\
\hline
\end{tabular}

TABLE XXXIV. Results for $g_{1}$ for $Q^{2}=2.4 \mathrm{GeV}^{2}$.

\begin{tabular}{|c|c|}
\hline$x$ & $g_{1} \pm$ stat \pm syst \\
\hline 0.8675 & $-0.0019 \pm 0.0099 \pm 0.0035$ \\
0.8009 & $-0.0204 \pm 0.0152 \pm 0.0042$ \\
0.7392 & $0.0193 \pm 0.0157 \pm 0.0050$ \\
0.6826 & $0.0373 \pm 0.0185 \pm 0.0059$ \\
0.6307 & $0.0987 \pm 0.0216 \pm 0.0109$ \\
0.5834 & $0.0492 \pm 0.0224 \pm 0.0097$ \\
0.5402 & $0.1134 \pm 0.0250 \pm 0.0151$ \\
0.5010 & $0.1252 \pm 0.0266 \pm 0.0148$ \\
0.4652 & $0.0995 \pm 0.0282 \pm 0.0129$ \\
0.4327 & $0.1138 \pm 0.0298 \pm 0.0118$ \\
0.4031 & $0.0976 \pm 0.0316 \pm 0.0142$ \\
0.3762 & $0.0754 \pm 0.0331 \pm 0.0126$ \\
0.3516 & $0.1392 \pm 0.0328 \pm 0.0179$ \\
0.3291 & $0.0952 \pm 0.0330 \pm 0.0154$ \\
0.3085 & $0.1645 \pm 0.0367 \pm 0.0255$ \\
\hline
\end{tabular}


TABLE XXXV. Results for $g_{1}$ for $Q^{2}=3.0 \mathrm{GeV}^{2}$.

\begin{tabular}{|c|c|}
\hline$x$ & $g_{1} \pm$ stat \pm syst \\
\hline 0.8868 & $-0.0137 \pm 0.0082 \pm 0.0088$ \\
0.8280 & $-0.0130 \pm 0.0120 \pm 0.0023$ \\
0.7723 & $0.0101 \pm 0.0129 \pm 0.0027$ \\
0.7202 & $0.0263 \pm 0.0148 \pm 0.0062$ \\
0.6715 & $0.0322 \pm 0.0172 \pm 0.0071$ \\
0.6262 & $0.0336 \pm 0.0184 \pm 0.0051$ \\
0.5844 & $0.0679 \pm 0.0208 \pm 0.0094$ \\
0.5457 & $0.0602 \pm 0.0226 \pm 0.0075$ \\
0.5101 & $0.0295 \pm 0.0238 \pm 0.0053$ \\
0.4772 & $0.0739 \pm 0.0244 \pm 0.0086$ \\
0.4470 & $0.0938 \pm 0.0254 \pm 0.0137$ \\
0.4191 & $0.1182 \pm 0.0267 \pm 0.0166$ \\
0.3935 & $0.1301 \pm 0.0275 \pm 0.0190$ \\
0.3699 & $0.0796 \pm 0.0303 \pm 0.0162$ \\
0.3481 & $0.0585 \pm 0.1017 \pm 0.0288$ \\
\hline
\end{tabular}

TABLE XXXVI. Results for $g_{1}$ for $Q^{2}=3.5 \mathrm{GeV}^{2}$.

\begin{tabular}{|c|c|}
\hline$x$ & $g_{1} \pm$ stat \pm syst \\
\hline 0.9033 & $0.0095 \pm 0.0070 \pm 0.0045$ \\
0.8515 & $-0.0064 \pm 0.0113 \pm 0.0025$ \\
0.8017 & $0.0082 \pm 0.0128 \pm 0.0032$ \\
0.7541 & $0.0235 \pm 0.0139 \pm 0.0039$ \\
0.7089 & $0.0125 \pm 0.0163 \pm 0.0049$ \\
0.6663 & $0.0499 \pm 0.0175 \pm 0.0068$ \\
0.6263 & $0.0495 \pm 0.0190 \pm 0.0077$ \\
0.5888 & $0.0786 \pm 0.0205 \pm 0.0148$ \\
0.5537 & $0.0629 \pm 0.0221 \pm 0.0082$ \\
0.5211 & $0.0763 \pm 0.0225 \pm 0.0131$ \\
0.4906 & $0.0931 \pm 0.0237 \pm 0.0148$ \\
0.4624 & $0.1116 \pm 0.0258 \pm 0.0169$ \\
0.4361 & $0.0239 \pm 0.0305 \pm 0.0110$ \\
\hline
\end{tabular}




\section{APPENDIX C}

DATA TABLES FOR $A_{1}, g_{1} / F_{1}$ AND $\Gamma_{1}$ 
TABLE XXXVII. Results for $g_{1} / F_{1}$ for $E=1 . x \mathrm{GeV}$.

\begin{tabular}{|c|c|c|}
\hline$Q^{2}\left(\mathrm{GeV}^{2}\right)$ & $x$ & $g_{1} / F_{1} \pm$ stat \pm syst \\
\hline 0.1010 & 0.1750 & $-0.1538 \pm 0.0498 \pm 0.0276$ \\
0.1200 & 0.1750 & $-0.1320 \pm 0.0385 \pm 0.0228$ \\
0.1440 & 0.1750 & $-0.1621 \pm 0.0351 \pm 0.0271$ \\
0.1710 & 0.1750 & $-0.1186 \pm 0.0355 \pm 0.0203$ \\
0.2050 & 0.1750 & $-0.0064 \pm 0.0202 \pm 0.0155$ \\
0.2440 & 0.1750 & $0.0321 \pm 0.0160 \pm 0.0173$ \\
0.2920 & 0.1750 & $0.0757 \pm 0.0132 \pm 0.0202$ \\
0.3480 & 0.1750 & $0.0978 \pm 0.0137 \pm 0.0218$ \\
0.1200 & 0.2500 & $-0.0889 \pm 0.0658 \pm 0.0251$ \\
0.1440 & 0.2500 & $-0.1124 \pm 0.0421 \pm 0.0235$ \\
0.1710 & 0.2500 & $-0.1857 \pm 0.0302 \pm 0.0321$ \\
0.2050 & 0.2500 & $-0.1480 \pm 0.0157 \pm 0.0249$ \\
0.2440 & 0.2500 & $-0.1243 \pm 0.0118 \pm 0.0208$ \\
0.2920 & 0.2500 & $-0.0597 \pm 0.0122 \pm 0.0151$ \\
0.3480 & 0.2500 & $0.0534 \pm 0.0117 \pm 0.0188$ \\
0.4160 & 0.2500 & $0.1007 \pm 0.0117 \pm 0.0230$ \\
0.4960 & 0.2500 & $0.1408 \pm 0.0163 \pm 0.0261$ \\
0.2050 & 0.3500 & $-0.0506 \pm 0.0365 \pm 0.0231$ \\
0.2440 & 0.3500 & $-0.0926 \pm 0.0212 \pm 0.0207$ \\
0.2920 & 0.3500 & $-0.1267 \pm 0.0129 \pm 0.0216$ \\
0.3480 & 0.3500 & $-0.1433 \pm 0.0113 \pm 0.0222$ \\
0.4160 & 0.3500 & $-0.0970 \pm 0.0123 \pm 0.0165$ \\
0.4960 & 0.3500 & $-0.0395 \pm 0.0150 \pm 0.0177$ \\
0.5920 & 0.3500 & $0.0733 \pm 0.0187 \pm 0.0241$ \\
0.2440 & 0.5000 & $0.0095 \pm 0.0551 \pm 0.0457$ \\
0.2920 & 0.5000 & $-0.0095 \pm 0.0299 \pm 0.0321$ \\
0.3480 & 0.5000 & $-0.0602 \pm 0.0207 \pm 0.0241$ \\
0.4160 & 0.5000 & $-0.1104 \pm 0.0133 \pm 0.0208$ \\
0.4960 & 0.5000 & $-0.1139 \pm 0.0127 \pm 0.0186$ \\
0.5920 & 0.5000 & $-0.1138 \pm 0.0123 \pm 0.0169$ \\
\hline & & \\
\end{tabular}


TABLE XXXVIII. Results for $g_{1} / F_{1}$ for $E=5 . x \mathrm{GeV}$.

\begin{tabular}{|c|c|c|}
\hline$Q^{2}\left(\mathrm{GeV}^{2}\right)$ & $x$ & $g_{1} / F_{1} \pm$ stat \pm syst \\
\hline 1.0000 & 0.1750 & $0.1034 \pm 0.0411 \pm 0.0183$ \\
1.1900 & 0.1750 & $0.0644 \pm 0.0413 \pm 0.0168$ \\
1.4400 & 0.1750 & $0.1221 \pm 0.0362 \pm 0.0204$ \\
1.0000 & 0.2500 & $0.1484 \pm 0.0411 \pm 0.0217$ \\
1.1900 & 0.2500 & $0.1466 \pm 0.0415 \pm 0.0218$ \\
1.4400 & 0.2500 & $0.1053 \pm 0.0222 \pm 0.0174$ \\
1.7100 & 0.2500 & $0.1307 \pm 0.0236 \pm 0.0201$ \\
2.0500 & 0.2500 & $0.0993 \pm 0.0306 \pm 0.0208$ \\
1.0000 & 0.3500 & $0.1829 \pm 0.0631 \pm 0.0249$ \\
1.1900 & 0.3500 & $0.1371 \pm 0.0618 \pm 0.0252$ \\
1.4400 & 0.3500 & $0.1946 \pm 0.0366 \pm 0.0246$ \\
1.7100 & 0.3500 & $0.2002 \pm 0.0320 \pm 0.0227$ \\
2.0500 & 0.3500 & $0.1885 \pm 0.0288 \pm 0.0250$ \\
2.4400 & 0.3500 & $0.1865 \pm 0.0249 \pm 0.0265$ \\
2.9200 & 0.3500 & $0.1955 \pm 0.0396 \pm 0.0334$ \\
1.0000 & 0.5000 & $0.0915 \pm 0.0697 \pm 0.0150$ \\
1.1900 & 0.5000 & $0.1577 \pm 0.0714 \pm 0.0237$ \\
1.4400 & 0.5000 & $0.2515 \pm 0.0463 \pm 0.0294$ \\
1.7100 & 0.5000 & $0.2829 \pm 0.0393 \pm 0.0363$ \\
2.0500 & 0.5000 & $0.2916 \pm 0.0351 \pm 0.0339$ \\
2.4400 & 0.5000 & $0.2797 \pm 0.0328 \pm 0.0305$ \\
2.9200 & 0.5000 & $0.2283 \pm 0.0297 \pm 0.0292$ \\
3.4800 & 0.5000 & $0.2660 \pm 0.0358 \pm 0.0424$ \\
4.1200 & 0.5000 & $0.2518 \pm 0.0721 \pm 0.0423$ \\
1.4400 & 0.7500 & $-0.0562 \pm 0.0857 \pm 0.0138$ \\
1.7100 & 0.7500 & $-0.0622 \pm 0.0746 \pm 0.0199$ \\
2.0500 & 0.7500 & $0.1252 \pm 0.0718 \pm 0.0229$ \\
2.4400 & 0.7500 & $0.2400 \pm 0.0670 \pm 0.0334$ \\
2.9200 & 0.7500 & $0.1804 \pm 0.0624 \pm 0.0298$ \\
3.4800 & 0.7500 & $0.2928 \pm 0.0684 \pm 0.0496$ \\
4.1200 & 0.7500 & $0.4343 \pm 0.0641 \pm 0.0613$ \\
\hline & & \\
\end{tabular}


TABLE XXXIX. Results for averaged $A_{1}$ for $Q^{2}$ between 1.4 and $4.52 \mathrm{GeV}^{2}$ and $W>1.6 \mathrm{GeV}$.

\begin{tabular}{|c|c|}
\hline$x$ & $A_{1}^{d} \pm$ stat \pm syst \\
\hline 0.1750 & $0.1324 \pm 0.0431 \pm 0.0202$ \\
0.2250 & $0.0856 \pm 0.0261 \pm 0.0185$ \\
0.2750 & $0.1493 \pm 0.0242 \pm 0.0249$ \\
0.3250 & $0.2062 \pm 0.0246 \pm 0.0294$ \\
0.3750 & $0.2456 \pm 0.0275 \pm 0.0354$ \\
0.4250 & $0.2880 \pm 0.0332 \pm 0.0403$ \\
0.4750 & $0.3626 \pm 0.0396 \pm 0.0450$ \\
0.5250 & $0.3704 \pm 0.0524 \pm 0.0507$ \\
0.5750 & $0.4148 \pm 0.0658 \pm 0.0611$ \\
0.6250 & $0.4629 \pm 0.0963 \pm 0.0594$ \\
0.6750 & $0.7891 \pm 0.1530 \pm 0.1015$ \\
\hline
\end{tabular}


TABLE XL. Results for $\Gamma_{1}^{d}$ integrated up to the values in $W$ given in table VI (measured region).

\begin{tabular}{|c|c|}
\hline$Q^{2}\left(\mathrm{GeV}^{2}\right)$ & $\Gamma_{1}^{d}$ (meas) \pm stat \pm syst \\
\hline 0.0496 & $-0.0118 \pm 0.0052 \pm 0.0024$ \\
0.0592 & $-0.0136 \pm 0.0040 \pm 0.0013$ \\
0.0707 & $-0.0092 \pm 0.0039 \pm 0.0017$ \\
0.0844 & $-0.0195 \pm 0.0040 \pm 0.0030$ \\
0.1010 & $-0.0171 \pm 0.0041 \pm 0.0024$ \\
0.1200 & $-0.0193 \pm 0.0043 \pm 0.0041$ \\
0.1440 & $-0.0183 \pm 0.0045 \pm 0.0043$ \\
0.1710 & $-0.0259 \pm 0.0045 \pm 0.0063$ \\
0.2050 & $-0.0187 \pm 0.0029 \pm 0.0038$ \\
0.2440 & $-0.0161 \pm 0.0023 \pm 0.0030$ \\
0.2920 & $-0.0125 \pm 0.0020 \pm 0.0025$ \\
0.3480 & $-0.0104 \pm 0.0018 \pm 0.0029$ \\
0.4160 & $-0.0083 \pm 0.0016 \pm 0.0025$ \\
0.4960 & $-0.0064 \pm 0.0016 \pm 0.0017$ \\
0.8440 & $0.0156 \pm 0.0067 \pm 0.0034$ \\
1.0100 & $0.0196 \pm 0.0062 \pm 0.0035$ \\
1.2000 & $0.0265 \pm 0.0058 \pm 0.0042$ \\
1.4400 & $0.0228 \pm 0.0035 \pm 0.0032$ \\
1.7100 & $0.0217 \pm 0.0026 \pm 0.0028$ \\
2.0500 & $0.0212 \pm 0.0022 \pm 0.0027$ \\
2.4400 & $0.0190 \pm 0.0017 \pm 0.0023$ \\
\hline
\end{tabular}


TABLE XLI. $\Gamma_{1}^{d}$ in the measured region plus the DIS contribution given by our model.

\begin{tabular}{|c|c|}
\hline$Q^{2}\left(\mathrm{GeV}^{2}\right)$ & $\Gamma_{1}^{d}$ (full $) \pm$ stat \pm syst \\
\hline 0.0496 & $-0.0141 \pm 0.0052 \pm 0.0024$ \\
0.0592 & $-0.0164 \pm 0.0040 \pm 0.0014$ \\
0.0707 & $-0.0125 \pm 0.0039 \pm 0.0018$ \\
0.0844 & $-0.0234 \pm 0.0040 \pm 0.0030$ \\
0.1010 & $-0.0216 \pm 0.0041 \pm 0.0025$ \\
0.1200 & $-0.0244 \pm 0.0043 \pm 0.0041$ \\
0.1440 & $-0.0239 \pm 0.0045 \pm 0.0044$ \\
0.1710 & $-0.0317 \pm 0.0045 \pm 0.0063$ \\
0.2050 & $-0.0251 \pm 0.0029 \pm 0.0053$ \\
0.2440 & $-0.0226 \pm 0.0023 \pm 0.0051$ \\
0.2920 & $-0.0181 \pm 0.0020 \pm 0.0050$ \\
0.3480 & $-0.0146 \pm 0.0019 \pm 0.0052$ \\
0.4160 & $-0.0101 \pm 0.0017 \pm 0.0050$ \\
0.4960 & $-0.0050 \pm 0.0016 \pm 0.0045$ \\
0.8440 & $0.0133 \pm 0.0067 \pm 0.0049$ \\
1.0100 & $0.0191 \pm 0.0062 \pm 0.0049$ \\
1.2000 & $0.0278 \pm 0.0058 \pm 0.0055$ \\
1.4400 & $0.0264 \pm 0.0035 \pm 0.0048$ \\
1.7100 & $0.0289 \pm 0.0026 \pm 0.0043$ \\
2.0500 & $0.0316 \pm 0.0022 \pm 0.0040$ \\
2.4400 & $0.0334 \pm 0.0017 \pm 0.0035$ \\
\hline
\end{tabular}




\section{VITA}

Kahanawita G. Vipuli G. Dharmawardane

Department of Physics

Old Dominion University

Norfolk, VA 23529

Vipuli Dharmawardane is from Colombo, Sri Lanka. She did her undergraduate studies at the University of Colombo, Sri Lanka, where she graduated with a Bachelor of Science in physics in 1996. She was accepted in the physics graduate program at Old Dominion University in 1997. She defended her PhD dissertation on April 1, 2004.

Typeset using $\mathrm{HAT}_{\mathrm{E}} \mathrm{X}$. 